\title{
Lean Personalized Medicine?
}

Citation for published version (APA):

Mahlmann, L. (2018). Lean Personalized Medicine? From genetic reductionism towards holistic Personalized Medicine. [Doctoral Thesis, Maastricht University]. Datawyse / Universitaire Pers Maastricht. https://doi.org/10.26481/dis.20180710lm

Document status and date:

Published: 01/01/2018

DOI:

10.26481/dis.20180710lm

Document Version:

Publisher's PDF, also known as Version of record

\section{Please check the document version of this publication:}

- A submitted manuscript is the version of the article upon submission and before peer-review. There can be important differences between the submitted version and the official published version of record.

People interested in the research are advised to contact the author for the final version of the publication, or visit the DOI to the publisher's website.

- The final author version and the galley proof are versions of the publication after peer review.

- The final published version features the final layout of the paper including the volume, issue and page numbers.

Link to publication

\footnotetext{
General rights rights.

- You may freely distribute the URL identifying the publication in the public portal. please follow below link for the End User Agreement:

www.umlib.nl/taverne-license

Take down policy

If you believe that this document breaches copyright please contact us at:

repository@maastrichtuniversity.nl

providing details and we will investigate your claim.
}

Copyright and moral rights for the publications made accessible in the public portal are retained by the authors and/or other copyright owners and it is a condition of accessing publications that users recognise and abide by the legal requirements associated with these

- Users may download and print one copy of any publication from the public portal for the purpose of private study or research.

- You may not further distribute the material or use it for any profit-making activity or commercial gain

If the publication is distributed under the terms of Article $25 \mathrm{fa}$ of the Dutch Copyright Act, indicated by the "Taverne" license above, 

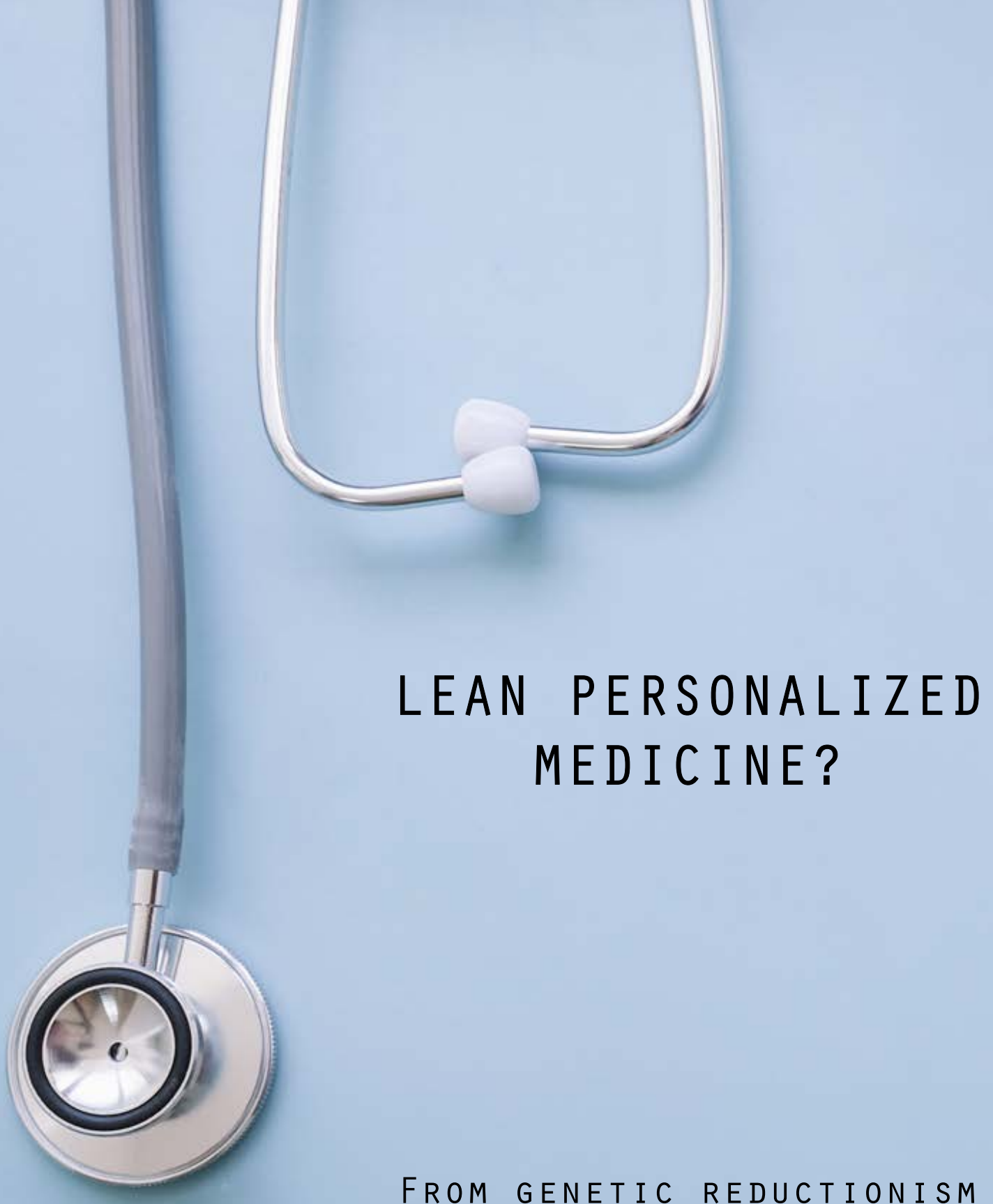

FROM GENETIC REDUCTIONISM TOWARDS

HOLISTIC PERSONALIZED MEDICINE

LAURA MÄHLMANN 
C c copyright Laura Mählmann, Maastricht 2018

Printing: Datawyse | Universitaire Pers Maastricht 


\section{Lean Personalized Medicine?}

\section{From genetic reductionism towards holistic Personalized Medicine}

\section{Dissertation}

to obtain the degree of Doctor at Maastricht University on the authority of the Rector Magnificus Prof. Dr. Rianne M. Letschert in accordance with the decision of the Board of Deans, to be defended in public on the $10^{\text {th }}$ of July 2018 at $12: 00$

by

Laura Mählmann

born July $18^{\text {th }}, 1988$ in Oldenburg, Germany 


\section{Supervisor:}

Prof. Dr. Angela Brand

\section{Co-Supervisor:}

Prof. Dr. Markus Gerber, University of Basel, Switzerland

\section{Assessment Committee:}

Prof. Dr. Leopold Curfs (Chairman)

Prof. Dr. Shyama Ramani

Dr. Peter Schröder-Bäck

Prof. Dr. Undine Lang, University Hospital of Basel, Switzerland

Dr. Nora Benhabiles, Alternative Energies and Atomic Energy Commission (CEA), France 


\section{Content}

$\begin{array}{ll}\text { Preface } & 7\end{array}$

Background 9

Introduction 11

Aim of this Dissertation 21

Outline 23

Part 1 Developing Awareness and Empowerment 25

Chapter 1 Attitudes towards personal genomics among older Swiss adults:

$\begin{array}{ll}\text { An exploratory study } & 29\end{array}$

Part 2 Integrating Big Data and ICT Solutions 47

Chapter 2 Big Data for Public Health Policy-Making: Policy Empowerment 51

Part 3 Translating Basic to Clinical Research and Beyond 67

Chapter 3 Psychological wellbeing and physical activity in children and adolescents with inflammatory bowel disease compared to healthy controls

Chapter 4 Impaired objective and subjective sleep in children and adolescents with inflammatory bowel disease compared to healthy controls

Chapter 5 Aerobic exercise training in children and adolescents with inflammatory bowel disease: Influence on psychological functioning, sleep and physical performance An exploratory trial

Chapter 6 Favorable impact of acute and chronic aerobic exercise training on disease symptoms and cardiovascular fitness in children and adolescents with inflammatory bowel disease

Part 4 Bringing Innovation to the Market 
Chapter 8 Personalized Medicine: What's in it for Rare Diseases?

Discussion \& Future perspectives

Valorisation

Acknowledgements

About the author

Publications 


\section{Preface}

This dissertation represents my progression of understanding the complexity of the human body, the multidimensionality of the system trying to care for this human body and the nature of change.

It is a progression officially starting in early 2016 . However, in fact, it already started 2013 during my master studies at Maastricht University when I met Prof. Dr. Angela Brand for the first time in her office. At this first meeting, I was confronted with and inspired by her map of new disease classifications, her description of the multidimensional and interdisciplinary challenges involved and her vision of tomorrow's world of understanding health and disease. I soon developed the motivation to scientifically explore this field in more detail, which led to the conduction of this dissertation. With the following presentation of cumulative findings, I intend to shed light on a variety of fields that play a significant role in the transition towards holistic and maybe lean Personalized Medicine. 



\section{Background}

Today's world is facing a hype of personalizing virtually anything [1]: Your name on your coffee cup at Starbucks; the "Share a Coke" campaign in 2014, when Coke bottles were printed with the 250 most popular names of today's teenager generation and Amazon recommending books that like-minded people purchased $[2,3]$.

The aim of personalization is to take all interests and preferences of the customer into account, create a unique and memorable experience and to give him or her the feeling of being at the center of attention. Especially in western societies this notion of individualism and the rise of empowered customers have been particularly highly developed [4]. The independence and uniqueness of the individual grew into focus of media, arts and science [5]. With the information revolution, increasing amounts of information on individual needs and behavior patterns became available and enabled the improved accountability of services, reduced costs and new means to tailor services [6,7].

But how has the trend of personalization affected the theory and practice of medicine? The increase of and improvements in informatics, sequencing technologies and computer sciences are enabling a revolution in medical practice [10]. These developments led to new scientific knowledge on molecular leveled data and contributed to new discoveries and classifications of human diseases $[1,9]$. As a result, personalization in medicine is a concept aiming to tailor therapy based on molecular and genetic stratification to ensure improved treatment outcomes $[1,10]$. The promises of so called "Personalized Medicine" (PM) have been met by much enthusiasm among researchers; however, realization is facing multiple challenges.

To amplify these challenges with scientific investigations, original data, as well as new lines of thought, defines the importance of this work. It will be further emphasized in the collection of publications forming the core of this dissertation. In the following, the topic will be introduced in more detail: The current state of medicine will be described, the potential and critical aspects of a more personalized approach will be illustrated and a description of current political action related to PM will be given. It will be followed by the definition on the main research question and an outline of this work. 



\section{Introduction}

\section{Current state of medicine}

Treatments and services in medicine are founded in the concept of traditional evidence based medicine (EBM). As defined by David Sackett (1997), "the practice of evidence-based medicine means integrating individual clinical expertise with the best available external clinical evidence from systematic research" [11]. "Individual clinical expertise" describes the judgment competency that health professionals obtain through clinical experience. "Best available external clinical evidence" in turn refers to a wide variety of patient-centered medical science that are consulted to improve the accuracy and precision in diagnostics and treatment decisions. As a consequence, the initial aim of EBM was to improve medical treatment of individual patients by applying the most efficacious interventions and therefore improve quality of life and longevity, while lowering costs [11].

However, the approach of EBM has its limitations. For instance, it was proposed that practicing EBM would be informed by a broad range of research output. Yet, reality shows that constantly evaluating the growing and fast changing body of evidence is a very time consuming process and much of the available data is irrelevant and invalid to clinical practice [12]. As a mean of preselecting applicable sources of evidence, randomized controlled trials (RCTs) and systematic reviews became the "golden standard" to inform clinical decision making and judging treatment options [11]. However, because classical clinical RCTs, are conducted in artificial scientific settings and harvest only a handful of measurements from a small and homogeneous group of patients, again, the informative value of results might be limited $[13,14]$. As a consequence, diagnosis and treatment are largely based on population averages and do not take into account the individual (molecular) characteristics of the patients. Experience shows that applying average effects to individual cases is potentially harmful and might even lead to adverse effects, exacerbating treatable problems and resulting in increased hospitalization $[15,16]$. For example, on a daily bases, millions of people take medication that will either have no effect or that are potentially unsafe for certain ethnic groups $[17,18]$, due to the bias towards white western participants in the safety assessment trials. In the case of HIV drugs STORCRIN ${ }^{\circledR}$ and 
SUSTIVA $®$ (efavirenz) differences in the pace of metabolizing the drug have been observed. Especially in people of African heritage a reduced speed of metabolizing the drug led to accumulations and toxic overdoses, as compared to European populations [19]. In contrary, too fast elimination of the body due to very active metabolism might result in no effect at all [20].

Consequently, several drugs have been evaluated regarding their efficacy and numerous have failed to improve the conditions they are supposed to ameliorate. Concrete examples are ABILIFY® (aripiprazole) for schizophrenia [21], NEXIUM® (esomeprazole) for heartburn [22], HUMIRA® (adalimumab) for arthritis [23], CRESTOR® (rosuvastatin) for high cholesterol [24], CYMBALTA® (duloxetine) for depression [25], ADVAIR DISKUS ${ }^{\circ}$ (uticasone propionate) for asthma [26], ENBREL $®$ (etanercept) for psoriasis, REMICADE® (iniximab) for crohn's disease [27], COPAXONE $\circledast$ (glatiramer acetate) for multiple sclerosis [28] and NEULASTA® (peglgrastim) for neutropenia [29]. The following figure by Schork published in Nature illustrates efficacy profiles of the previous examples [13] (fig. 1).

In sum, this variation in treatment effects reflect diversity in risk profiles for disease and variable responsiveness to treatment that include vulnerability to toxicity and could lead to adverse effects [13,30]. Health professionals considered factors including severity and activity of the disease, interactions with additional drugs, as well as the patient's age, nutritional status and renal or liver function [31]. However, despite the importance of these factors, new scientific insights uncovered inherited differences in the metabolism, absorption, excretion and genetic polymorphisms in drug therapy patients that might affect the efficacy and safety of a drug [31,32]. Tailoring drug treatment to such relevant factors would not only improve the effectiveness of drugs and reduce side effects, but might also improve treatment compliance. Since patient non-compliance remains another leading factor of adverse health effects and exacerbation of conditions, especially with regard to chronic diseases [33].

In conclusion, the current EBM approach is limited in predicting how treatments, especially drugs, might affect a patient whose age, comorbidities, on top treatments and genetics were excluded from the population demographics for the tested drug [10]. The question is whether this "one size fits all approach" could be improved by a more individualized approach taking unique patient characteristics into account [30]. 


\section{IMPRECISION MEDICINE}

For every person they do help (blue), the ten highest-grossing drugs in the

United States fail to improve the conditions of between 3 and 24 people (red).

\section{ABILIFY (aripiprazole)}

Schizophrenia

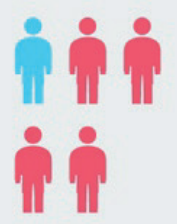

3. HUMIRA (adalimumab) Arthritis

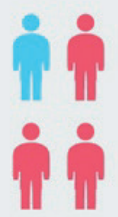

5. CYMBALTA (duloxetine) Depression

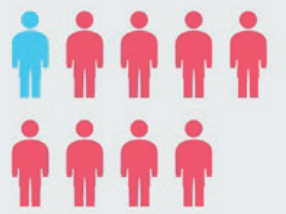

\section{REMICADE (infliximab)} Crohn's disease

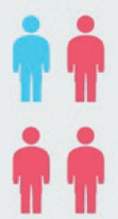

\section{NEXIUM (esomeprazole)}

Heartburn

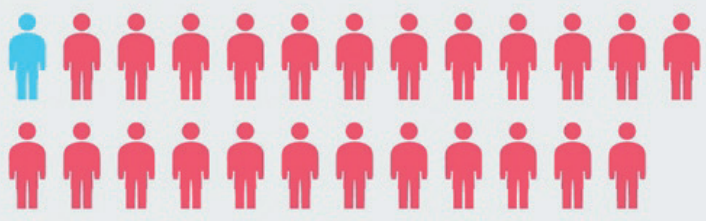

4. CRESTOR (rosuvastatin)

High cholesterol

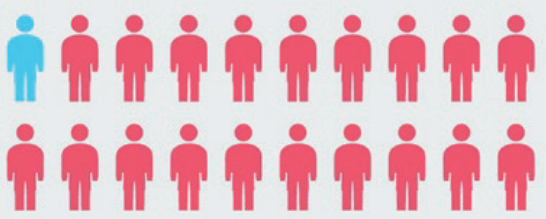

6. ADVAIR DISKUS (fluticasone propionate) Asthma

7. ENBREL (etanercept) Psoriasis

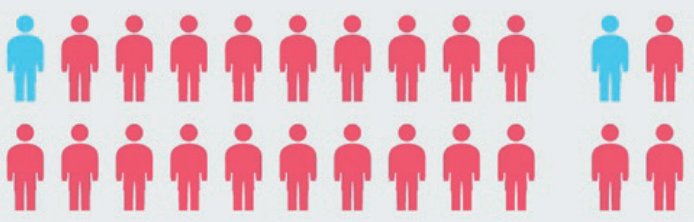

9. COPAXONE (glatiramer acetate) Multiple sclerosis

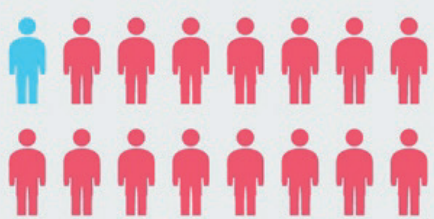

10. NEULASTA (pegfilgrastim) Neutropenia

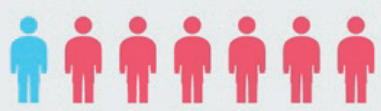

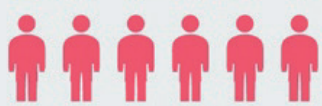

Based on published number needed to treat (NNT) figures. For a full list of references, see Supplementary Information at go.nature.com/4dr78f.

Figure 1: Efficacy profiles of different commonly used drugs [13] 


\section{Aim of Personalized Medicine}

In recent years, as the shortcomings of the EBM model of medicine on the one hand and the potential for more individually-tailored and personalized treatments on the other have become more apparent, major investments to better understand inter-individual differences have created opportunities for significant improvements [1]. Researchers have discovered distinct inherent genetic traits that contribute to human diseases or cause variation in response to therapy [34]. To describe the concept of Personalized Medicine (PM), multiple terms such as genomic medicine, stratified medicine, and precision medicine are used interchangeably. Those terms give a sense of great medical advances even if they do not fully clarify what PM means [35]. Until now, the majority of experts reduce the concept of PM to treatments that are extended using on genetic analysis and "static" biomarkers [36], while others describe PM as an approach in which the healthcare professional bases his or her treatment on the patients' health status and individual circumstances [37].

For the purpose of this dissertation, the definition of PM used by the European Commission Horizon 2020 Advisory Group for Societal Challenge, "Health, Demographic Change and Wellbeing" is applied. This definition has been selected since it represents the European Perspective and tries to incorporate a holistic approach of PM:

'Personalized Medicine refers to a medical model using characterisation of individuals' phenotypes and genotypes (e.g. molecular profiling, medical imaging, lifestyle data) for tailoring the right therapeutic strategy for the right person at the right time, and/or to determine the predisposition to disease and/or to deliver timely and targeted prevention' [38].

\section{The End of "One Size Fits All"}

The first step towards increased individualistic characterization of patients was the completion of the Human Genome Project (HGP) in 2003 [39]. Key findings of the project included the identification of 1800 disease genes. However, in contrast to the expectation that these genes would be the key to understanding human health and disease [40], results of the HGP showed that, from a genetic point of view, the genetic make-up of all humans is $99.1 \%$ identical, while interindividual genetic variability in human species only account for a variability of $0.9 \%$ [39]. Since this 0.9 percentile correspond to three billion base pairs, potential for significant inter-individual genetic variability is still high [39]. Within the inter-individual different $0.9 \%$ of the human genome, single nucleotide polymorphisms (SNPs) are the changes in single nucleotides and account for $90 \%$ of all 
known genetic polymorphisms that lead to inter-individual differences $[41,42]$. The ability to identify these polymorphisms enables the detection of molecular signatures, which characterizes patient susceptibility to various diseases. The detection of these molecular signatures allows for specified diagnosis and more accurate predictions of responsiveness to drug therapy. Many of these discoveries have been obtained through genome wide association studies (GWAS). The GWAS approach compares the genetic profiles of patients with a certain disease to those of healthy controls through rapid scanning of markers across the complete set of the DNA including genetic variations associated with a particular disease [43]. These broadband comparisons have been made possible by microarray biochip technology that helps to store and analyze the patients' genomes efficiently, and thereby revolutionizing the practice of human genome sequencing $[42,44-47]$.

\section{Application of Personalized Medicine today}

Innovative technologies and the advancements in human genome research were a significant step towards modeling and customizing medical treatments. Incorporating genetics, molecular characteristics and clinical profiles could help determine an optimized and personalized treatment approach for the individual patient [48]. The intention of today's model of PM is to identify predispositions and early screening of biomarkers, to diagnose and to prognose based on genetic markers, to define the right drug dosage and to integrate lifestyle into medical treatment procedures. In detail, first, PM aims to identify a patient's predisposition to specific disease through genome analysis and therefore, supports decision making about preemptive intervention. Currently, there are more than 350 genetic tests available to identify patients with increased risks to develop diseases [48]. Screening for predispositions holds value in identifying at risk patients early to prevent them from disease and economic burden later on. Well-known examples are mutations of the BRCA1 and BRCA2 genes that have been implicated in familial breast cancers [49], LRRK2 predictor for Parkinson's [50] and the loss of APC gene function in familial adenomatous polyposis [51].

Second, in line with the identification of genetic predisposition, PM aims to screen for biomarkers of disease in advance to clinical manifestations and thus allow for earlier treatment and decreased morbidity and mortality [52]. For example, a variety of biomarkers have been identified to predict primary and secondary tumor risks [53]. They are usually products of cancerous cells, which are excreted in blood or urine. This is of high importance since sometimes non-cancerous conditions can cause increasing levels of tumor markers or, in contrary, not every cancer will express with raised levels of tumor markers. Therefore, awareness 
and monitoring of these additional biomarkers provide opportunity to improve management and efficiency of treatment at an early stage [54]. Screening in the sense of predicting before the disease occurs, still seems more difficult to realize and several biomarkers are currently evaluated as potential screening tests for early malignancies. These include the use of alpha-fetoprotein (AFP) in screening for hepatocellular cancer in high-risk subjects, cancer antigen (CA) 125 in combination with transvaginal ultrasound (TVU) in screening for epithelial ovarian cancer, prostate-specific antigen (PSA) in screening for prostate cancer, faecal occult blood testing (FOBT) in screening for colorectal cancer (CRC) and vanillymandelic acid and homovanillic acid in screening for neuroblastoma in newborn infants [55].

Third and fourth, PM aims to base diagnosis and prognosis on patient-specific genetic characteristics rather than on population average data [56]. Again in oncology, a wide variety of biomarkers have been identified to support molecular diagnosis and prognosis. For example, first elevated human epidermal growth factor receptor type 2 (Her2) in breast cancer predict resistance to endocrine and chemotherapy treatment but susceptibility to Her2-targeted treatment [57]. Second, elevated estrogen receptor levels instead correlate with increased endocrine response but result in chemotherapy resistance [58]. While many cancer screens utilize DNA analysis, in the case of cervical cancer screening DNA testing can only detect the presence of HPV, which is insufficient for characterization, since only with protein examination it can be determined whether HPV is active or latent $[59,60]$.

Fifth, PM aims to assist clinicians in finding the ideal drug dosage for their patients. As illustrated in figure 1, many commercially available drugs on the market work for only a small percentage of the people who take it [61] and many patients are facing a long and costly journey of trial-and-error prescriptions. PM has recently begun to link different drug responses to differences in the coding genes for drug-metabolizing enzymes, drug transporters, or drug targets [62]. For Warfarin, three markers are at play: CYP2C9 is the gene that encodes the enzyme responsible for Warfarin metabolism; VKORC1 has been identified as a gene encoding the enzyme that recycles vitamin $\mathrm{K}$ (the drug's primary target); and CYP4F2 is a gene encoding the enzyme responsible for vitamin $\mathrm{K}$ metabolism [41]. Based on this knowledge, the US Food and Drug Administration (FDA) recently recommended genotyping for all patients receiving Warfarin to avoid adverse drug effects [62].

Sixth, PM aims to include lifestyle data to recognize the personal circumstance and lifestyle effects on the patients' health and disease. The emergence of selfmonitoring tools, such as a wristband accelerometer, will empower people to take charge of their own health by providing info, support and control. However, the integration of lifestyle into daily medical practice is still very limited [63]. 
In sum, genetics and other molecular screening enable the physician to select a more accurate prescription in time and lead to improved treatment outcomes for the patient $[62,64]$. However, adoption is slow and some experts express their criticism as described in more detail in the next paragraph.

\section{Critical Aspects of Personalized Medicine}

In addition to the promising opportunities and potential of PM, some experts point towards critical aspects that should be considered. First, based on experience, all major advances in health care come from public health initiatives focusing on population wide programs. These include clean water programs, effective sewage systems, food safety regulations, environmental pollutants monitoring projects, vaccination programs, maternity services and public lifestyle recommendations [40]. As stated by Interlandi (2016) "Life span has increased by about a decade in the past 50 years. And none of that gain is related to DNA. It's learning about smoking and diet and exercise. It's old fashioned stuff" [65]. Therefore, some are questioning, whether personalization is really beneficial for society as a whole, especially in a system, in which health and social insurance programs are embedded in principles of solidarity and social citizenship [40]. With PM, the query of injustice and inequity in health treatments may play a significant role again. Even though costs for genome sequencing are decreasing, highly individualized treatments may result in unforeseen additional costs. Further, reimbursement strategies of health insurance providers are highly dependent on the potential for economic return and therefore hinder health insurance providers from being able to take such an altruistic focus as to invest in the benefit of patients at such an individual level $[66,67]$. Pharmaceutical companies on the other hand might decide to invest even more into the most valuable markets, leading to increased global inequality and racial segmentation [40].

Second, from a legal point of view, current developments such as medical online services, predictive potential of genetics and Big Data are creating challenges that legal systems are not yet prepared for. Questions such as data protection against genetic discrimination and general informed consent are still in discussion [68]. These issues challenge deeply embedded values of society. And even though the promises of PM might be revolutionary, the question of whether everything that might become possible should be applied will need to be carefully considered.

Third, while highly accurate diagnostic and prognostic information might be of tremendous value for medical care, patients would have to confront the realities of life-altering diseases. The psychological impact of such information and the influence of religious or moral tenets has to be considered [69]. In line, some 
argue, that medicine cannot be personalized in a setting that ignores patients specific needs for respect and emotions [70]. Therefore, some suggested to integrate information gained from complementary and alternative medicine to reach a truly holistic approach [70]. In this context, critical voices advise PM to consider the question how to balance the need for technologically driven science and society's wish for a holistic approach in medical care.

Finally, related to the wish for more holistic approaches in medical care, critique has been expressed about the underestimated complexity of genetics. Therefore, best practices in clinical settings are still rare and genetic information is not yet available that would facilitate the targeted dosages, disease identification or targeted therapy. For example, as previously described, the frequently prescribed blood thinner Warfarin is known for its narrow therapeutic range [41]. PM has recently begun to link different drug responses to differences in the coding genes for drug-metabolizing enzymes, drug transporters, or drug targets [62]. However, it turns out that Warfarin is still highly dependent on environmental factors including diet [41]. In addition to the difficulty of drug targeting, the complexity of identifying diseases has been underestimated. Different genetic causes can lead to a similar disease phenotypes classified by classical symptoms. The discovery of single genes that encode for diseases was limited and the focus shifted towards GWAS to identify genetic associations. Furthermore, the utility of targeted therapy for common chronic diseases, such as cardiovascular, neurological and autoimmune diseases with their attached complexities and constrains is still limited. In sum, the application of PM seems so far inadequate due to highly complex interactions of many genes of small effect $[40,71]$ and the significant influence of environmental and biographical factors [72].

\section{Need for a holistic perspective in Personalized Medicine}

Taking previous critique into account, the field of opportunities will increase if the complex matrix of interactions between many potential factors leading to disease will be acknowledged. Clinical medical practices may remain much more than stratification along genetic lines. However, genetics is a central element, as well as the notion that many diseases are rooted in human biology and genes. Genetic testing should not be decisive and prioritized over other forms of clinical knowledge, but might serve to reinforce the complexity of disease categories. In addition, high throughput 'omics' technologies do not just enable the extraction of information on DNA variations in genomics, but also enable the analysis of peptides and proteins (proteomics) and of intermediate products of the metabolism (metabolomics) and many other contributing factors [73]. Finally, the external 
microorganisms residing in the gut, known as the microbiome, are receiving increasing attention in their significant contribution to human health and disease [73].

Through a better understanding of the applied 'omics' technologies increased awareness of environmental factors and the acknowledgement of personal values, a more personalized approach to medicine holds real promise $[74,75]$. The benefits of such an approach could enable more effective early diagnosis, less toxic treatments for patients, improved medical services to citizens, and improving the overall health of the population [38]. Furthermore, the current predictive ability is expected to progress through innovation in various technologies and will enable even further improvements in health outcomes overtime [35].

\section{Europe's Perspective: The Five PerMed SRIA Challenges}

On a political level, the European Commission is a major driver of PM across Europe, recognizing the potential opportunities and seeking to address the challenges. Since 2007, various reports have been published that analyzed the potential for, and issues with, the use of 'omics' technologies in PM. These reports assess recent developments in EU legislation for placing medicinal products and devices on the market; and investigate factors affecting the uptake of PM in health care systems [76-78]. In support, the EU has committed over 3.2 billion Euro to medical research related to PM [79]. Furthermore, the EU funded Coordination and Support Action (CSA) PerMed developed 2013-2015 the 'Strategic Research and Innovation Agenda (SRIA)', which outlined a roadmap for actions at national as well as EU-level: 'Shaping Europe's Vision for Personalized Medicine' [38]. Overall, the agenda includes general recommendations and proposes research activities which could pave the way for the further implementation of PM and ultimately aim to support a paradigm shift among all stakeholders. The outlined research and recommendations are based on the analysis of strategic reports, interviews and consultations with experts and representatives of all relevant sectors in the field of PM.

The aim of the SRIA is to "coordinate efforts between EU stakeholders and find solutions to overcome fragmented activities, insufficient communication and lack of generic solutions to realize the true potential of PM within Europe" [38]. To reach this goal, the consortium identified the following five key challenges $[38,80]$ in order to facilitate further implementation of PM:

Challenge 1: Developing Awareness and Empowerment

Challenge 2: Integrating Big Data and Information and Communication Technology (ICT) Solutions 
Challenge 3: Translating Basic to Clinical Research and Beyond

Challenge 4: Bridging Innovation to the Market

Challenge 5: Shaping Sustainable Health Care

In figure 2 the five challenges are illustrated in a "Circle of Challenges" together with important enablers and stakeholders. The overall aim of PM research and implementation is indicated at the center of the circle.

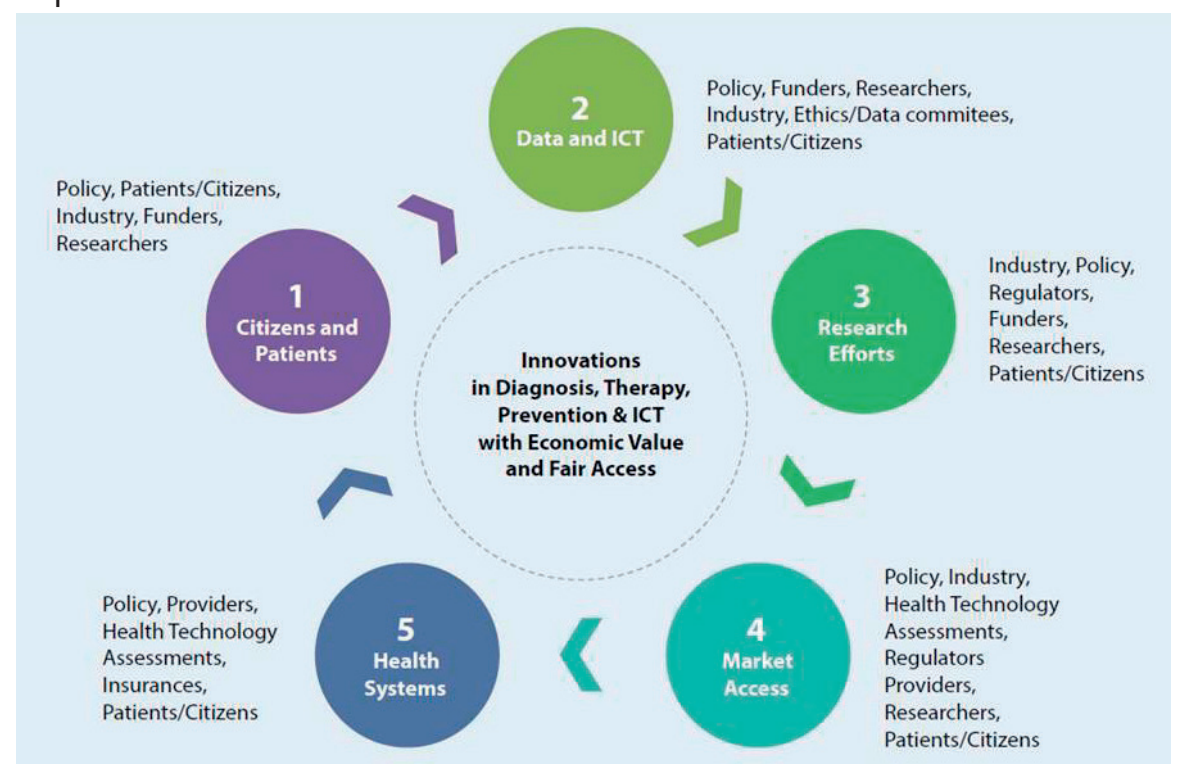

Figure 2: Circle of Challenges [9] 


\section{Aim of this Dissertation}

PM is an innovative concept in medicine that entails many challenges yet to be solved. These challenges have been well described by the 'Strategic Research and Innovation Agenda (SRIA)', developed by the EU funded Coordination and Support Action (CSA) PerMed [38]. Intention of the challenges is to foster implementation of PM and to promote a paradigm shift among stakeholders towards a more holistic systems thinking approach in Personalized Medicine. This holistic approach would broaden PM's analytical scope and increase its potential for informing more accurate treatment. In the present dissertation, the five SRIA challenges have been chosen as a frame of orientation and horizontal guideline. Each challenge will be translated into actionable research objectives aiming:

To shed light on translational aspects, in order to move from a genetic focus towards more holistic systems thinking in Personalized Medicine.

The research conducted as part of this dissertation will provide scientific evidence and original data to the PerMed SRIA challenges. The data aims to deliver insights on the clinical, personal and economical utility of PM and enable a better understanding on how the changes brought by PM will benefit the individual patient as well as society. Further, gaps of knowledge will be identified that need to be addressed. 



\section{Outline}

This work is organized in an introduction and five parts, according to the five SRIA challenges. Each part will commence with a short introduction explaining the SRIA challenges in more detail. Second, a specific field of investigation and a research objective will be defined. Furthermore, the related publication and method will be introduced. This will be followed by an abstract and the publications as officially published and organized as indicated in this outline. In total, five scientific peer reviewed articles with an impact factor (IF), one submitted article, one book chapter and one business plan will form the core of this work.

Briefly summarized, Part 1 refers to SRIA Challenge 1: "Developing Awareness and Empowerment". For this challenge access to genetic data and genetic data sharing form the specific field of investigation. An exploration of people's awareness, attitudes, motivation and concerns about personal genomic testing, accessing genetic data and genetic data sharing will be presented in Chapter 1 , a quantitative survey study.

Part 2 refers to SRIA Challenge 2: "Integrating Big Data and ICT Solutions". The need to utilize the potential of Big Data and the role of Big Data in the movement towards PM is frequently discussed. In Chapter 2, an opinion article will illustrate the potential of data driven policy making to reach the EU's key health policy objective of strengthening the health care systems' effectiveness, accessibility, resilience, quality and performance.

Part 3 refers to SRIA Challenge 3: "Translating Basic to Clinical Research and Beyond". A next step towards the integration of PM is to take a closer look at cases of chronic complex diseases, as well as at the effect of lifestyle changes in treatment success. Chapter 3 to 6 will document a clinical case control study investigating children and adolescents with inflammatory bowel diseases, a chronic complex disease, and the potential of an innovative lifestyle intervention for treatment improvement.

Part 4 will illustrate SRIA Challenge 4: "Bringing Innovation to the market". In Chapter 7, a business plan "MoVit" exemplifies how innovative mobile health applications can support transition towards PM.

Part 5 refers to SRIA Challenge 5 - "Shaping sustainable Healthcare". In chapter 8 , a book chapter will discuss a new paradigm in disease categorization and methods of approaching cases of rare diseases in PM. Furthermore, this 
chapter will shed light on the consequences for the health care system and policy making.

Finally, the discussion will summarize the cumulative findings and discuss the impact of PM. Further, this section will review the strengths and limitations of PM, which should foster and guide future research. An outlook will be given to raise awareness of the continuous development of technology and the need for a dynamic paradigm in PM as well as a conclusion on the main research question. 


\section{Part 1}

Developing Awareness and Empowerment

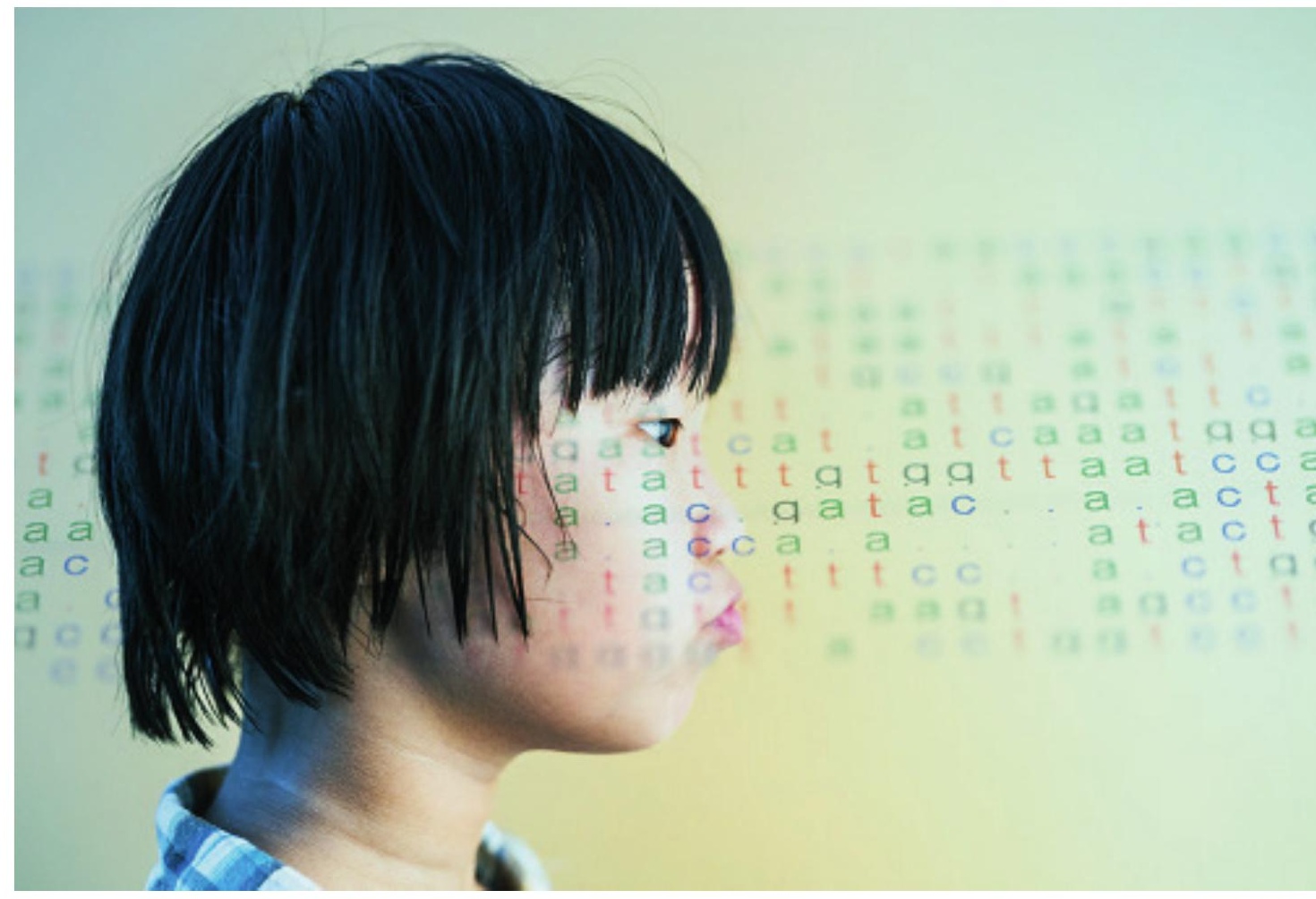


Part 1 
With the advent of PM, the role of caregivers and patients will evolve. Successful implementation of PM will be achieved only if all stakeholders, including patients and healthcare professionals, are empowered and develop the required awareness about PM. [...] Models that enable sharing, ownership and the development of a sense of responsibility towards personal health data, as well as the improvement of PM health literacy, will need to be generated along with suitable common principles, appropriate policy and regulatory frameworks. Public engagement in PM can be increased by enabling citizens to become actively involved in all phases of research and development ('citizen science'). [...]

SRIA, Challenge 1 [38]

Innovation in treatment and therapy is currently raising challenges for health care professionals and patients alike. A multitude of new information, tools and technologies are emerging, giving access to new layers of information in the complexity of disease. Furthermore, these new tools and technologies are directly accessible for patients [81]. Therefore, access to new information, the potential for patient-empowerment that it entails and the literacy of patients to actually understand this information are key issues in this challenge.

One illustrative example for direct to consumer accessible medical technology is Personal Genomics (PG): the procedure of sequencing, analysis and interpretation of an individuals' genome [82]. Overall, genetic information is one of the cornerstones of the development and implementation of PM [83]. As previously mentioned, the development in 'omics' technologies has led to a more detailed understanding of the complexity of diseases, which allows a more accurate classification of diseases based on their molecular characteristics [84]. Consequently, these tests could soon play an integral role in the delivery of health care by providing information that possibly will form the basis for profound life decisions.

With the falling prices and less time needed to sequence the human genome, some companies have developed commercial services based on these technologies and have started to sell direct-to-consumer (DTC) genetic tests $[82,85]$. Direct-to-consumer Personal Genomics (DTC-PG) are sold via the internet, television, or other marketing venues without involving health care professionals [86]. Such testing provides consumers with a personalized risk assessment for a variety of complex diseases, genetic traits, ancestry, and pharmacogenomics information [85]. However, the analytical validity, reliability and clinical utility of such tests are highly contested and significant debate has surrounded these companies ever since they first emerged [82,87-89]. Experts' views differ over whether this type of genomic risk information has any value for disease prevention. Beyond that, there is a severe lack of scientific evidence regarding the impact of personal genomic information on behaviour and health outcomes [90]. The 


\section{Part 1}

number of people who receive such information and who lack the genetic literacy to appropriately respond to it is still unknown [91].

Given the overall need for genomic data to receive further insights into the complex system of health and disease, the interest that people show in accessing their genomic data and even donating them for research, as well as the concerns about the practices of many personal genomics providers lead to the first objective of this dissertation:

Objective (1): To investigate the societal perception of research participation, the value people attribute to genetic research and the types of tradeoffs between privacy and utility that people are willing to accept.

This objective has been addressed by one peer reviewed scientific publication by Mählmann et al. [82] entitled "Attitudes towards personal genomics among older Swiss adults: An exploratory study" (Chapter 1). The publication reports findings of a quantitative survey study among 151 older Swiss adults. 


\section{Chapter 1}

\section{Attitudes towards personal genomics among older Swiss adults: \\ An exploratory study}

Published as: MähImann L, Röcke C, Brand A, Hafen E, Vayena E: Attitudes

towards personal genomics among older Swiss adults:

An exploratory study. Appl Transl Genomics 2016;8:9-15. [IF:0.94] 


\section{Chapter 1}

\section{Abstract}

Objectives: To explore attitudes of Swiss older adults towards personal genomics (PG).

Methods: Using an anonymized voluntary paper-and-pencil survey, data were collected from 151 men and women aged 60-89 years attending the Seniorenuniversität Zurich, Switzerland (Seniors' University). Analyses were conducted using descriptive and inferential statistics.

Results: One third of the respondents were aware of PG, and more than half indicated interest in undergoing PG testing. The primary motivation provided was respondents' interest in finding out about their own disease risk, followed by willingness to contribute to scientific research. Forty-four percent were not interested in undergoing testing because results might be worrisome, or due to concerns about the validity of the results. Only a minority of respondents mentioned privacy-related concerns. Further, $66 \%$ were interested in undergoing clinic-based PG motivated by the opportunity to contribute to scientific research $(78 \%)$ and $75 \%$ of all study participants indicated strong preferences to donate genomic data to public research institutions.

Conclusion: This study indicates a relatively positive overall attitude towards personal genomic testing among older Swiss adults, a group not typically represented in surveys about personal genomics. Genomic data of older adults can be highly relevant to late life health and maintenance of quality of life. In addition they can be an invaluable source for better understanding of longevity, health and disease. Understanding the attitudes of this population towards genomic analyses, although important, remains underexamined. 


\section{Introduction}

The emergence of increasingly inexpensive genome sequencing technologies has increased our potential to identify genetic components of health and disease, thereby contributing to progress towards more individualized, predictive, preventive and precise medicine (Van El et al., 2013). To facilitate this development, genome-wide association and gene-environment interaction studies have become valuable resources for investigating the genetic basis of disease.

Capitalizing on hopes and expectations regarding personalized medicine, commercial companies began offering large-scale personal genomic testing and disease-risk profiling outside clinical settings in 2007 . Such companies appeared in many countries, operating via online interfaces and initially using a direct-toconsumer approach (DTC) whereby users order tests online and receive personal predictive risk assessments for a variety of diseases directly, without the mediation of a health professional (Vayena and Prainsack, 2013). However, the analytical validity, clinical utility of such tests including the appropriate involvement of health care providers have been highly contested, and significant debate has surrounded these companies ever since their first emergence (Bloss et al., 2013; Vayena et al., 2014a; Vayena, 2014). The leading company in this area remains 23andme, claiming to have one million genotypes in their database (23andme, 2015). Since November 2013, at the request of the US FDA, the company can offer only limited health risk information depending on whether the FDA has been provided with sufficient evidence of the link between a variant and a condition. In the UK, the same company offers a wider range of health risk information as the UK regulators have not stepped into controlling the services.

Expert views differ over whether this type of genomic risk information has any value for disease prevention or would only inappropriately burden individuals and the healthcare system (Bloss et al., 2013 \& McGowan et al., 2014). Systematic evidence of the impact of personal genomic information on behavior and health outcomes remains limited. Overall, early findings indicate increased physician utilization by DTC-PG customers (Bloss et al., 2014). Healthier lifestyle changes on the basis of genomic results was only reported by Egglestone et al., (2013), while others observed no changes or even decreased self-efficacy and control over one's health (Bloss et al., 2013; Boeldt et al., 2015). Further, differences in anxiety levels or after test distress in DTC-PG users were reported as nonsignificant and correct interpretation and understanding of PG findings varies with demographic characteristics, level of education and numeracy, genetic knowledge and the format in which the information is presented (Ostergren et al., 2015; Schmidlen et al., 2015). Most findings come from early adopters of DTCPG. We are still lacking conclusive evidence of how lay people receiving complex genomic information would react to it, given the limited genomic literacy in the 
general public (Carere et al., 2015; Critchley et al., 2015a,b; Boeldt et al., 2015 Meisel et al., 2015; Ostergren et al., 2015 Schmidlen et al., 2015; Gigerenzer et al., 2007; Gigerenzer and Muir Gray, 2011; Wasson et al., 2013).

To date a number of studies have sought to improve understanding of the attitudes of people who have accessed their genomic data, or those willing to do so (Carere et al., 2015; Roberts and Ostergren, 2013; Vayena et al., 2012; Vayena et al., 2014a). While patterns have begun to emerge, empirical data remain limited (Vayena et al., 2014a): findings indicate that the general public's awareness about genomics is increasing, even though the number of actual test takers remains low (Almeling and Gadarian, 2014; Carere et al., 2015; Critchley et al., 2015a, b; Hall et al., 2012; Schmidlen et al., 2015). The most commonly reported motives for undergoing personal genomic testing outside the clinical context are to obtain information about personal traits; to explore genetic makeup and ancestry; or wanting to learn about individual genetic risk factors (Cherkas et al., 2010; Gollust et al., 2012; McGowan et al., 2010; Vayena et al., 2012; Vayena et al., 2014a). Other reasons include the use of genetic information for disease prevention (Rahm et al., 2012); planning to adopt healthier lifestyles in cases of higher-than-average risk (Cherkas et al., 2010); explanation for the cause of a current health condition (Meisel et al., 2015) and taking personal responsibility for future health (McGowan et al., 2010). An additional reason has been the opportunity to contribute to research, despite privacy and confidentiality concerns being reported as obstacles to the donation of data (Brown Trinidad et al., 2010; Critchley et al., 2015a, b).One study found people accepting trade-offs between privacy and the utility of research participation for the common good (McGuire et al., 2009; Oliver et al., 2012). However, conditions for such trade-offs or expectations associated with utility are still an underexplored area. Since we anticipate an increase in the use of genomic data within and beyond the clinical setting, it is important to fill gaps in empirical data on public attitudes concerning these issues.

Following up on previous work we did on PG attitudes in Switzerland, this study focuses on investigating perspectives of older Swiss adults. In most western societies the older adult population is the fastest growing segment; medical advances and the baby boom between 1955 and 1964 have resulted in a current population in which $1 / 6$ of people are 65 or older, with a steadily increasing percentage aged 80 or older (BFS 2013). Compared to the general population, older adults are more vulnerable to physical changes, decreasing mobility, chronic illness, loss of support systems, and changes in cognitive ability, particularly in advanced old age. However, they are also characterized by large inter-individual differences in level, rate and direction of change of these characteristics. In the context of genetic research, given their long life history of health and disease, they also represent an extremely rich source of insight into genetic associations 
with human longevity, as well as genetic associations leading to individual fitness (van den Akker et al., 2015; Tan et al., 2006).

In general, older adults are frequently underrecruited or excluded from biomedical and pharmaceutical research, although most drugs and treatments under development are more likely to be used in this age group. The elderly perspective is also missing from the discussion of personal genomics, since studies have mainly focused on younger populations with concerns corresponding to earlier phases in life where health issues are typically a concern of the future, as opposed to the more current concern of many older adults (Smith and Freund, 2002; Wurm et al., 2009). This project aimed to start filling the gap in our knowledge regarding attitudes of older adults towards human genomics. More specifically, we wanted to explore whether this age group is aware of developments within genetic testing, in particular personal genomics; their interest in undergoing such testing themselves; the motivational factors contributing to their willingness or lack thereof; how they view access modalities to such data; and their concerns and expectations in relation to participating in genomics research. To our knowledge this is the first study of this kind.

\section{Method}

We conducted an anonymized voluntary self-completion survey in two sessions on 19 November 2013 and 6 March 2014, before lectures of the Seniors' University Program at the University of Zurich with approximately 500 older adults attending on the first date, and 300 on the second date. After a short presentation of the study purpose, we showed a multimedia file including a soundless black and white animation and text describing the customer journey of internet-based personal genomic services. The file was developed in collaboration with the IT support group of the University of Zurich, and its content, design and presentation were reviewed by experts in genetics, psychology, social science, medicine, law, ethics, and information technology. The same multimedia file was used in a study we conducted with young adults and results of which have been published (Vayena et al., 2014a). After the presentation of the movie, the audience was invited to ask questions of clarification about the movie and the project. They were also invited to pick up a paper-pencil questionnaire, including a prepaid return envelope, which was designed based on our previous study of similar research questions in young adults (Vayena et al., 2012, 2014a), and after reviewing similar questionnaires in other published studies (Vernez et al., 2013; Wilde et al., 2011; Gollust et al., 2012). Permission to conduct this study was received by the Research Ethics Committee of the Canton of Zurich. 


\section{Chapter 1}

The questionnaire included 31 multiple-choice and 7-point Likertscale questions and was pretested among peers and five older adults unrelated to the study. Questions were designed to capture awareness about personal genomics services, motivation for testing, and concerns about genomic results. Moreover, a number of questions were included that explored attitudes towards sharing genomic data and research participation in genomic research studies. Since the majority of respondents were unlikely to have had experience with personal genomic testing, we also explored the motives and concerns of hypothetical participation in testing. Demographic data were collected including gender, age, education, health concerns, offspring, importance of religion, and internet usage.

Statistical analysis was carried out using SPSS Statistics 20.0. We conducted Pearson's chi-squared test and, where required, Fisher's exact test in order to establish associations between attitudes to personal genomics and the demographic variables. Due to our small sample, the 7-point-Likert scale scores were split into groups for comparison of different measures by pooling answer categories 1-3 and 5-7. The neutral category, 4, was excluded. Again, analysis was conducted and analyzed using Pearson's chi-squared or Fisher's exact test. Significance was accepted at $\alpha=0.05$ level. Participants' answers to open-ended questions were reported in free text fields for "other reasons" or additional comments. These were post-coded into categories and analyzed qualitatively or saved as illustrating quotations.

\section{Results}

In total, 151 respondents participated in the survey, a response rate of $19 \%$ based on the total number of attendees at the lecture. General sample characteristics are displayed in Table 1. Gender was fairly equally distributed and the mean age reported was 76 years, with a range between 60 and 89 years. The level of education was above average, and $92 \%$ of our sample reported regular internet use. Participants expressed moderate concern about their own health, with 4.89 on a 7-point Likert-scale, and one third reported being affected by a serious disease either personally or within their family. The importance of religion in daily life seemed less prominent. One third of participants reported having heard of personal genomic testing. Pearson's chi- squared test revealed that the oldest age group (born 1925-1935) was less likely to be aware of DTC-PG $\left(X^{2}(1, n=84)=\right.$ $6.025, p=0.024)$, and also reported less internet use $\left(x^{2}(1, n=84)=19.704, p\right.$ $=0.0$. 
Direct-to-consumer personal genomics (DTC-PG): motivation to undergo testing

Only one respondent had personal experience with DTC personal genomics; this person had participated in a research project. Of the remaining respondents, $56 \%$ were interested in hypothetically undergoing DTC-PG. The primary motivation for considering testing was interest in receiving information about one's personal disease risk, followed by the wish to contribute to scientific research (Table 2). The exploration of demographic characteristics and the willingness to undergo testing revealed that people concerned about their health were more likely to consider DTC-GT $\left(X^{2}(1, n=122)=5.348, p=0.021\right)$.

Table 1: Demographics.

\begin{tabular}{llll}
\hline Sample characteristic & & $\mathrm{N}$ & $\%$ \\
\hline Sample size & & 151 & \\
Age & Male & 76 years & SD $=6.052$ \\
Gender & Female & 82 & $54.3 \%$ \\
& Yes & 69 & $45.7 \%$ \\
Children/grandchildren & No & 126 & $83.4 \%$ \\
Disease in family & Yes & 25 & $16.6 \%$ \\
& No & 48 & $31.8 \%$ \\
Internet & Yes & 99 & $65.6 \%$ \\
& No & 139 & $92.1 \%$ \\
Education & Secondary school & 12 & $7.9 \%$ \\
& Vocational education & 7 & $4.6 \%$ \\
& High School & 28 & $44.4 \%$ \\
Health & University degree & 49 & $18.5 \%$ \\
& Concerned & 104 & $62.5 \%$ \\
Religion & Not concerned & 19 & $12.6 \%$ \\
& Religious & 38 & $25.2 \%$ \\
\hline
\end{tabular}

A $x^{2}$-test including all demographic characteristics was conducted and revealed the following results: People without children or grandchildren are less concerned about passing on predispositions $\left(X^{2}(1, n=84)=16.154, p=0.000\right)$; People without children or grandchildren are less likely to want to find out about their genetic ancestry $\left(X^{2}(1, n=84)=8.201, p=0.004\right)$; People with sickness in family are more likely to prefer having health professional supervision $\left(X^{2}(1, n=82)=\right.$ 4.353, $p=0.037$; Comparing the two extreme groups in terms of age, the oldest group (1935-1925) was less likely to prefer medical professional supervision $\left(X^{2}\right.$ $(1, n=50)=6.637, p=0.01)$. 


\section{Chapter 1}

Table 2: Ranked reasons for considering undergoing DTC

\begin{tabular}{lll}
\hline Response $(\mathrm{N}=84)$ & \multicolumn{2}{l}{$\begin{array}{l}\text { No of } \\
\text { responses }\end{array}$} \\
\hline I would like to know if I am at risk of certain disease & 59 & 70.2 \\
I would like to contribute my genetic data to scientific research & 53 & 63.1 \\
I would like to know my sensitivity to certain medication & 48 & 57.1 \\
I would like to find out about my genetic ancestry & 46 & 54.8 \\
I would like to know the risk that I have passed a disease predisposition on to my & 45 & 53.6 \\
children & 36 & 42.9 \\
I would like to find out about my genetic traits & 33 & 39.3 \\
I was curious about how such a test works & 30 & 35.7 \\
I am interested in genetics in general & 29 & 34.5 \\
If I were able to buy the test at low cost & 5 & 6 \\
Just for fun & & \\
\hline
\end{tabular}

\section{Reasons for not being interested in DTC-PG}

Those respondents who were not interested in undergoing DTC-PG (44\%) would refrain mainly because of concerns that the results might worry them $(45.5 \%)$ and concerns about the validity of the results $(42.4 \%$, see also Table 3$)$.

A $X^{2}$-test including the demographic variables was conducted and revealed the following results: Females see costs as an obstacle $\left(x^{2}(1, n=66)=3.960, p=\right.$ $0.047)$; People with sickness in family are less likely to report "no utility" ( $\chi^{2}(1, n=$ $64)=7.721, p=0.005)$ and would not undergo testing since it is not prescribed by the doctor $((1, n=64)=5.217, p=0.032)$; People in the oldest age group are less concerned about privacy $\left(X^{2}(1, n=47)=5.631, p=0.02\right)$ and religious people refrain since it is not prescribed by the doctor $\left(X^{2}(1, n=53)=6.280, p=0.019\right)$.

Table 3: Ranked reasons for not being interested DTC-PG.

\begin{tabular}{lll}
\hline Response $(\mathrm{N}=66)$ & $\begin{array}{l}\text { No of } \\
\text { responses }\end{array}$ & {$[\%]$} \\
\hline I am concerned that the results will worry me & 30 & 45.5 \\
I do not think the test results are valid & 28 & 42.4 \\
I do not see any utility in such tests/l am not interested in my genetic & 28 & 42.4 \\
profile & 18 & 27.3 \\
I am concerned about the privacy of my data & 14 & 21.2 \\
I am skeptical about genetic testing in general & 11 & 16.7 \\
Cost is an obstacle to undergo testing & 11 & 16.7 \\
The test is not advised by a doctor & & \\
\hline
\end{tabular}

\section{Attitudes towards online PG vs clinic-based PG for research purposes}

In contrast to $56 \%$ interested in an online PG test, $66 \%$ of the respondents were interested in participating in a clinic-based personal genomic test for research purposes, with those concerned about their health more likely to be interested $\left(X^{2}\right.$ 
$(1, n=122)=4.419, p=0.036)$. Overall, the main reason for interest was the opportunity to contribute to scientific research $(78 \%)$ and second, to help increase the validity of the test $(69 \%)$. More specifically, participants who had experienced disease personally or in their family were motivated by their interest in the development of new therapies $\left(X^{2}(1, n=97)=7.904, p=0.005\right)$, as well as improving the validity of available personal genomic tests $\left(X^{2}(1, n=97)=5.489, p=0.019\right)$. Participants with lower levels of education were less interested in developing therapies for affected family members or themselves $\left(x^{2}(1, n=100)=6.354, p=\right.$ $0.012)$, and also reported lower interest in genetics generally $\left(X^{2}(1, n=100)=\right.$ $6.135, p=0.013$ ).

Of those respondents expressing interest in undergoing clinic-based PG for research purposes, the vast majority reported an interest in receiving back their results, with $60 \%$ wanting unrestricted access to results and $30 \%$ wanting only to be informed about curable diseases. Only 4 respondents chose not to receive any results, because they doubted the validity of the results. An additional reason for refusal provided in the open-ended questions was the potential burden associated with more information about disease risk.

A $X^{2}$-test including the demographic variables was conducted and revealed the following results: people aware about genetic testing were less concerned about privacy $\left(x^{2}(1, n=47)=4.362, p=0.037\right)$. The same holds true for people with sickness in family $\left(x^{2}(1, n=47)=p=0.040\right)$. People without children or grandchildren reported quality concerns $\left(X^{2}(1, n=47)=, p=0.029\right)$.

Table 4: Ranked reasons for not considering a clinic-based PG for research purposes.

\begin{tabular}{lll}
\hline Response $(\mathrm{N}=50)$ & No of responses & {$[\%]$} \\
\hline I am concerned that the results will worry me & 23 & 46 \\
I am skeptical about genetic testing & 17 & 34 \\
I am concerned about the privacy of my data & 15 & 30 \\
I question the validity of the test & 9 & 18 \\
Time would be an obstacle to participating & 8 & 16 \\
I am not interested in genetics at all & 5 & 10 \\
\hline
\end{tabular}

One third of all respondents were not interested in participating in a clinical study overall worry about possible test results was cited as the key reason. The second most common reason was skepticism about genetic testing in general (see Table 4).

Finally, Fig. 1 illustrates reasons for not interested in undergoing either DTCPG or clinic-based PG for research purposes, indicating a common concern about the nature of the results. Low utility and low validity were only attributed to privately and self-administered DTC-PG. 


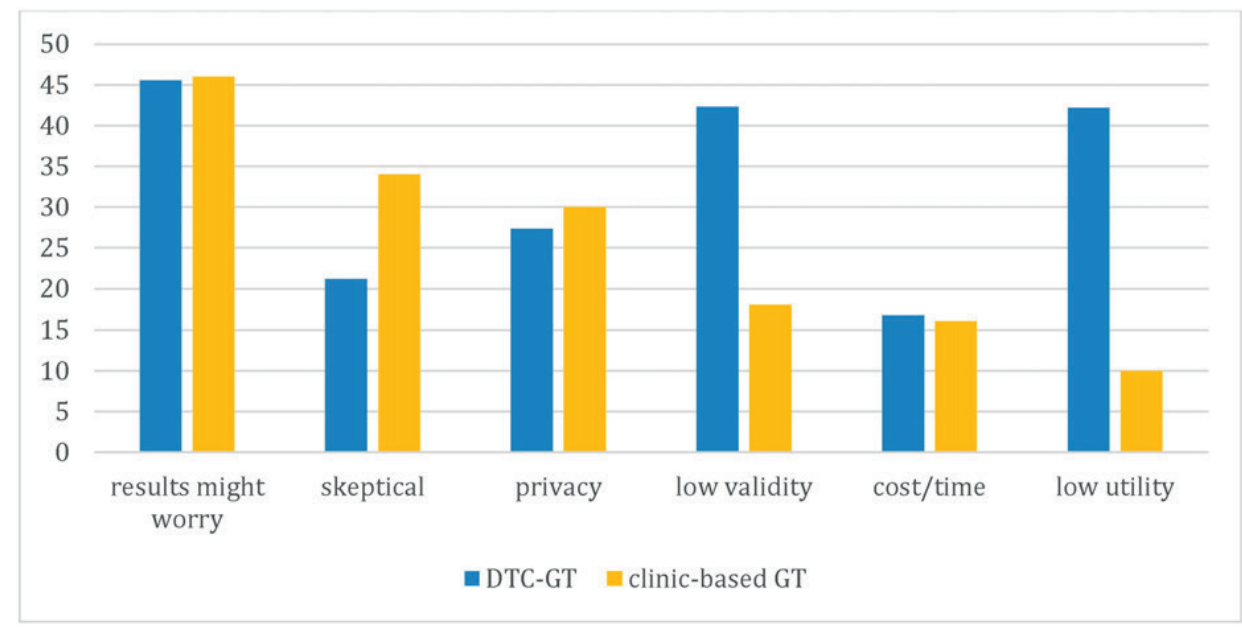

Figure 1: Comparison of reasons for not undergoing DTC-PG or clinic-based PG for research purposes. To enable comparison and adjust for varying total numbers, data have been normalized. Categories have been grouped and slightly renamed.

\section{Donating genomic data to research institutions}

Finally, $75 \%$ of all respondents indicated strong preferences to contribute their genomic data to public research institutions such as universities or university hospitals as opposed to private institutions (15\%). Forty-five percent would also support contributing data to private but not-for-profit institutions.

\section{Discussion}

\section{Awareness of personal genomic testing}

Some researchers have reported declining awareness of personal genomic testing with increasing age. Additionally, psychological and consumer behavior studies link increasing age with decreasing capacity for processing information from media sources (Ostergren et al., 2015; Kolor et al., 2012; Langford et al., 2012), which might lead to the reported lower awareness of PG in older people. Since some of the personal genomics services are online, it is also likely that the low internet use, especially among the oldest study participants of our group, might diminish awareness of online genomic services. However, a recent survey of internet use in older Swiss adults indicated that $68 \%$ of those aged $60-69$ and $40.6 \%$ of those aged 70 years and older reported using the internet to some degree (Schelling and Seifert, 2010).

In the present study, one third of participants were familiar with the concept of personal genomics, a proportion that can be classified as moderate compared to 
other studies involving young and middle-aged adults (Cherkas et al., 2010; Finney Rutten et al., 2012; Hall et al., 2012; Kolor et al., 2012; Ortiz et al., 2011). This finding is likely to be related to the increasing media attention and current public debate around a revision of the Swiss laws on genetic testing. In addition, one of the senior citizens' lectures used for recruitment was on innovations in medicine, which might imply pre-existing interest in the medical field within our sample.

\section{Reasons for DTC-PG-testing}

Only one person in the current study reported personal experience with genetic testing in the context of a genetic research study. This is in line with previous findings of a relatively low participation rate in personal genomic testing in other age groups (Kolor et al., 2012; Ortiz et al., 2011). More than half of our respondents expressed interest to participate in a hypothetical PG-study. Other studies characterized individuals with prior personal experience of a genetic disease in themselves or within their family as particularly open-minded towards genetic testing (Kettis-Lindblad et al., 2005); our findings, however, indicate more critical attitude to personal genomics within this group. Those with a family history of disease were particularly likely to question the utility of this type of test, and indicated that they would not undergo testing without involving a health professional.

Respondents were interested in access to information on personal disease risk and the opportunity to contribute to scientific research. Those respondents who were concerned about their health in general expressed a stronger interest in undergoing testing. Similarly, in the case of a clinic-based PG for research purposes, an additional motive for participation was to help increase the validity of the test. Other studies have reported analogous findings (Goldsmith and Jackson, 2012; Vayena, 2014): the moral duty of contributing — for reasons of reciprocity - to the societal good; altruism; and self-interest in medical treatment have all been described as common motivations to participate in genetic research for elderly groups (Frazier et al., 2006; Merz et al., 2002). Generally, altruism involves an act in which benefits are attributed to others rather than the actor; in genetic research, the recipient (a research institution), is considered to reciprocate the gift by contributing towards publicly accessible medical advances; therefore, a third party, society, eventually receives the benefits of the individual's altruistic act (Harmon and Chen, 2012). Erikson and Kivnick (1986) considered this type of altruism 'generativity': an increasing concern, with age, to guide and care for the next generation, coupled with greater willingness to provide positive opportunities for the next generation. Consequently, participation of the elderly may be seen by older adults themselves as a reciprocal act, whereby the older adult experiences personal benefits from participation, including increased or maintained life satisfaction, greater confidence and self esteem, as well feeling more 


\section{Chapter 1}

socially integrated through having contributed personally to the common good and/or the next generation (Harmon and Chen, 2012).

\section{Reasons for not being interested in PG-testing}

The primary reason for refraining from PG-testing was the possible implications of the results, which might cause worry (Bloss et al., 2010; Gollust et al., 2012; McGuire et al., 2009; Rahm et al., 2012; Vayena et al., 2014a). This finding relates to a rather prominent point in the debate about PG, namely the concern about whether people are able to understand the nature of genetic data and interpret predictive results correctly. Studies of investigating this question in the context of genetic literacy have had contradictory results. Some found that people lacked sophisticated knowledge about genetics and genomics while holding misconceptions about the value and limitations of genetic tests (Boeldt et al., 2015; Ostergren et al., 2015; Schmidlen et al., 2015; Hahn et al., 2010; Rose et al., 2005). Other studies found that participants have realistic expectations and understand the low predictive power of results (McGowan et al., 2010; McGuire et al., 2009; Meisel et al., 2015; Ostergren et al., 2015). It is therefore difficult to draw firm conclusions at this point about individual perceptions of actual test results. The risk of being surprised and unable to cope with probabilistic results might still occur in some instances, especially given the often observed limited ability of both doctors and patients to interpret probabilistic information about health risks correctly, even when those risks are unrelated to genetics (Boeldt et al., 2015; Carere et al., 2015, Dressler et al., 2014; Gigerenzer et al., 2007; Gigerenzer and Muir Gray, 2011, Kaye and Korf, 2013; Ostergren et al., 2015; Powell et al., 2012; Schmidlen et al., 2015). Those with a family history of disease were more likely to question the utility of this type of test, and indicated that they would not undergo testing without involving a health professional. Another interesting finding that emerged in the open questions was that some participants did not wish to be treated for illness after a certain stage; they were concerned, however, that having genetic information might lead to increased external pressure on them to undergo treatment despite their own wishes. Although the design of our study did not allow us to explore this further, we consider this an issue worthy of deeper investigation.

Given the prominence of privacy concerns in all discussions about genomic testing, privacy considerations were one of our possible reasons for not undergoing PG testing. Such concerns were very low among our respondents. A number of other studies have reported concerns about privacy, confidentiality of results and fear of discrimination as a key consideration in deciding whether or not to participate in genetic research (Critchley et al., 2015a, b; Vayena et al., 2014a; Goldsmith and Jackson, 2012; McGuire et al., 2009; Wilde et al., 2011). This is significantly different from our findings and should be explored in more detail. It 
is unclear whether lack of privacy concerns is due to a privacy knowledge gap or whether respondents in this study do not really consider genetic privacy an issue. Among the older participants in this study, privacy concerns were very rare, and diminished with increasing age. Given limited previous data on older adults' perception of privacy in the context of data sharing, we can only assume a number of reasons. First, many older adults might not be concerned about privacy because they are not affected by certain risks identified earlier: for example, they do not have employment concerns, they already have established (health) insurance plans, and they have already survived a substantial part of adult life. Second, in line with arguments relating to altruistic motives, older persons might consider the risk of diminished assurance of privacy due to data disclosure to be outweighed by the high potential benefits of scientific research (McGuire and Burke, 2008; Oliver et al., 2012; Vayena, 2014). Finally another reason that may potentially explain reduced privacy concerns, is the high level of privacy protection afforded to personal data in Switzerland (Vayena et al., 2014a). None of our respondents mentioned concerns about the privacy of their relatives in relation to their own genomic testing. A possible explanation could be low genomic literacy and limited understanding of the genetic privacy implications for relatives. The level of genomic literacy of this population and the perception of privacy risks need further exploration.

\section{Comparison of DTC-PG and clinic-based PG}

We compared participants' interest in undergoing DTC-PG and personal genomic testing in a clinical context for research purposes. According to Swiss law, for companies that operate from Switzerland, it is illegal to offer personal genomic services to consumers without physician prescription and counseling. It is not illegal for Swiss citizens to access such services online, but the ministry of health has issued advise against such online services. The genetic testing law is currently under revision, which includes relaxing some of these prohibitions, however, at the time of the survey no revision was effected. We were interested in exploring whether the context in which the genomic testing takes place is relevant in people's motivation or concerns. The comparison was most interesting in relation to the reasons for refraining from testing. As in the DTC-PG the primary reason was concerns that results may cause worry. Drawing on other findings from earlier studies, although in different age groups, we still observe that this concern persists as reasons of avoiding personal genomics irrespective of the context of testing. It is important to explore this sentiment towards genomic information in more depth. Is the underlying concern the result of a deterministic understanding of genomics, or is it a general attitude towards information with predictive value? Ultimately better understanding the reasons behind such concerns will also prove relevant for the design of genomic literacy activities. 


\section{Chapter 1}

\section{Not-for profit vs for-profit}

The vast majority of study participants indicated strong preference to donate data to public research institutions as opposed to private research institutions. Even though we did not further explore the underlying reasons for this preference, our hypothesis is that people trust not-for profit publicly funded organizations more for prioritizing the common good. Given the growing number of private for-profit companies operating in the space of genomic and personal data, it would be interesting to further explore such views also against people's broader notion of ownership and exploitation of genomic data.

\section{Limitations of the present study}

All participants in this study were recruited via the Seniorenuniversität Zurich, a continuing education program for seniors. These seniors are characterized by an above average level of education and high levels of social participation and engagement in physical, cultural and social activities. This profile implies that our sample represents a selected part of the aging population rather than being fully representative of the general population. The Seniorenuniversität itself claims that their members are 'the pioneers of a new culture of aging', and such a new culture is likely to involve higher levels of engagement in activities such as participating in research. Additionally, future generations of older adults will also include the socalled baby boom generation, with demographics more similar to the population in this survey.

The low response rate, $19 \%$, may indicate a substantial group of older adults outside the study with a critical perspective towards genetics, with only those holding a sufficiently strong preexisting interest in genetics and genomics actually participating.

We approached this sample in two sessions of a lecture series and introduced the topic by showing an informational multimedia file. We offered the change to pose clarifying questions, but we did not assess the viewers' comprehension of the video. The first time we approached the older adults was before a lecture on new developments in cardiology, a theme closely related to medical issues. This could imply an a priori increased interest in and awareness of new advances in medicine. Another limitation of the study is that due to the limited number of actual test takers in this age group, we investigated a hypothetical scenario by asking participants to imagine whether they would participate in genomic testing, and assessing their motivation for or against such participation. As our participants were aware of the hypothetical nature of this scenario, it is possible that motivational responses differed from those in a real-world test taking situation: the survey respondents might not have been as emotionally invested in the overall topic and in their decision as participants in a real-life personal genomic test. 
Despite the reported limitations, this study provides a first descriptive approach to unveiling attitudes and motives relating to personal genomics in later adulthood-a source of information largely untapped, but one that is likely to inform the current debate and approaches to policy making.

\section{Outlook and conclusion}

How to harness people's willingness to engage with their genomic data and contribute to research, while making sure they are not exploited and their individual rights are not being violated, remains a key challenge. As PG is a relatively new development we are only at the beginning of the debate about necessary measures, for example, at the regulatory level; how to engage the broader public in the debate; and ultimately how to protect individual rights, like privacy and confidentiality, while avoiding undue paternalism. Although these are global developments, policies tend to be developed and implemented at the national level and therefore they need to be responsive to different public perceptions about genomics, public trust in scientific and medical research, the health care systems, as well as different regulatory regimes. This exploratory study shows that older Western European adults of higher educational level and with familiarity with online technologies are generally interested in personal genomics. The participants were motivated by the possibility to participate in research and interest in one's actual and future health. This population is of particular impor- tance for genomic studies. Genomic data from this population can contribute to better understanding of longevity, gene-environment interactions and wellbeing. In addition, as this segment of the population is rapidly growing, more thought should be given on how to involve this group in genomic research. If that were to be the case, it is imperative to understand the attitudes, preferences and concerns of this population group in relation to genomic data. Such an understanding is crucial for developing appropriate policies and practices in relation to recruitment, informed consent and privacy-setting options relevant to personal genomics. We hope that our exploratory findings will help set a broader research agenda for genomics and senior adults.

\section{Acknowledgments}

We would like to thank the Seniorenuniversität (http://www.seniorenuni.uzh.ch/index. html ) for allowing us to conduct this study. We are grateful to Professor Martin Richards for very helpful comments on an early draft of the manuscript and to Christian Ineichen for reviewing the statistical analysis. 


\section{References}

1 Almeling, R., Gadarian, S., 2014. Public opinion on policy issues in genetics and genomics. GIM 491-494, http://dx.doi.org/10.1038/gim.2013.175.

2 Bloss, C.S., Ornowski, L., Silver, E., Cargill, M., Vanier, V., Schork, N.J., Topol, E.J., 2010. Consumer perceptions of direct-to-consumer personalized genomic risk assessments. Genet. Med. 12 (9), 556-566. http://dx.doi.org/10.1097/GIM.0b013e3181eb51c6.

3 Bloss, C.S., Wineinger, N.E., Darst, B.F., Schork, N.J., Topol, E.J., 2013. Impact of direct-toconsumer genomic testing at long term follow-up. J. Med. Genet. 50 (6), 393-400. http://dx.doi.org/10.1136/ jmedgenet-2012-101207 (Jun).

4 Bloss, C.S., Schork, N.J., Topol, E.J., 2014. Direct-to-consumer pharmacogenomic testing is associated with increased physician utilisation. J. Med. Genet. 51 (2), 83-89. http:// dx.doi.org/10.1136/jmedgenet-2013-101909 (Feb).

5 Boeldt, D.L., Schork, N.J., Topol, E.J., Bloss, C.S., 2015. Influence of individual differences in disease perception on consumer response to direct-to-consumer genomic testing. Clin. Genet. 87 (3), 225-232. http://dx.doi.org/10.1111/cge.12419 (Mar).

6 Brown Trinidad, S., Fullerton, S.M., Bares, J.M., Jarvik, G.P., Larson, E.B., Burke, W., 2010. Genomic research and wide data sharing: views of prospective participant. GIM 12, 486-495.

7 Bundesamt für Statistik, 2013. Informationen aus der Demografie. Newsletter Nr.1. March 2010 http://www.bfs.admin.ch/bfs/portal/de/index/news/publikationen.html?publicationlD=3864 Accessed 15 March 2014.

8 Carere, D.A., Kraft, P., Kaphingst, K.A., Roberts, J.S., Green, R.C., 2015. Consumers report lower confidence in their genetics knowledge following direct-to-consumer personal genomic testing. Genet. Med. http://dx.doi.org/10.1038/gim.2015.34 (Mar 26).

9 Cherkas, L.F., Harris, J.M., Levinson, E., Spector, T.D., Prainsack, B., 2010. A survey of UK public interest in internet-based personal genome testing. PLoS One $5 \mathrm{http} / / / \mathrm{dx}$.doi. org/10.1371/journal.pone.0013473.

10 Critchley, C., Nicol, D., Otlowski, M., Chalmers, D., 2015a. Public reaction to direct-to- consumer online genetic tests: Comparing attitudes, trust and intentions across commercial and conventional providers. Public Underst. Sci. 24 (6), 731-750. http://dx.doi.org/10.1177/0963662513 519937 (Aug).

11 Critchley, C., Nicol, D., Otlowski, M., 2015b. The impact of commercialisation and genetic data sharing arrangements on public trust and the intention to participate in biobank research. Public Health Genomics 18 (3), 160-172. http://dx.doi.org/10.1159/ 000375441.

12 Dressler, L.G., Jones, S.S., Markey, J.M., Byerly, K.W., Roberts, M.C., 2014. Genomics education for the public: perspectives of genomic researchers and ELSI advisors. Genet. Test. Mol. Biomarkers 18 (3), 131-140.

13 Egglestone, C., Morris, A., O'Brien, A., 2013. Effect of direct-to-consumer genetic tests on health behaviour and anxiety: a survey of consumers and potential consumers. J. Genet. Couns. 22, 565-575.

14 Erikson, J.M., Kivnick, H.Q., 1986. Vital Involvement in Old Age. Norton, NY.

15 Finney Rutten, L.J., Gollust, S.E., Naveed, S., Moser, R.P., 2012. Increasing public awareness of direct-to-consumer genetic tests: health care access, internet use, and population density correlates. J. Cancer Epidemiol. http://dx.doi.org/10.1155/2012/309109.

16 Frazier, L., Calvin, A.O., Mudd, G.T., Cohen, M.Z., 2006. Understanding of genetics among older adults. J. Nurs. Scholarsh. 38, 126-132.

17 Gigerenzer, G., Muir Gray, J.A., 2011. Better Doctors, Better Patients, Better Decisions: Envision Health Care 2, Better $=020$ (Eds.) MIT Press, Cambridge, MA.

18 Gigerenzer, G., Gaissmaier, W., Kurz-Milcke, E., Schwartz, L.M., Woloshin, S., 2007. Helping doctors and patients to make sense of health statistics. Psychol. Sci. Public Interest 8, 53-96. 
19 Goldsmith, L., Jackson, L., O'Connor, A., Skirton, H., 2012. Direct-to-consumer genomic testing: a systematic review of the literature on user perspectives. Eur. J. Hum. Genet. 20, 811-816.

20 Gollust, S.E., Gordon, E.S., Zayac, C., Griffin, G., Christman, M.F., Pyeritz, R.E., Wawak, L., Bernhardt, B.A., 2012. Motivations and perceptions of early adopters of personalized genomics: perspectives from research participants. Public Health Genomics 15, 22-30.

21 Hahn, S., Letvak, S., Powell, K., Christianson, C., Wallace, D., Speer, M., Lietz, P., Blanton, S., Vance, J., Pericak-Vance, M., Henrich, V.C., 2010. Genomedical connection: a communities' awareness and perceptions of genomic medicine. Public Health Genomics 13, 63-71.

22 Hall, T.O., Renz, A.D., Snapinn, K.W., Bowen, D.J., Edwards, K.L., 2012. Awareness and uptake of direct-to-consumer genetic testing among cancer cases, their relatives, and controls: The Northwest Cancer Genetics Network. Genet. Test. Mol. Biomarkers 16, 744-748.

23 Harmon, S.H.E., Chen, K.H., 2012. Medical research data-sharing: the 'public good' and vulnerable groups. Med. Law Rev. 20, 516-539.

24 Kaye, C., Korf, B., 2013. Genetic literacy and competency. Pediatrics 132 (Suppl. 3), S224S230.

25 Kettis-Lindblad, A., Ring, L., Viberth, E., Hansson, M.G., 2005. Genetic research and donation of tissue samples to biobanks. What do potential sample donors in the Swedish general public think? Eur. J. Pub. Health 16, 433-440.

26 Kolor, K., Duquette, D., Zlot, A., Foland, J., Anderson, B., Giles, R., Wrathall, J., Khoury, M.J., 2012. Public awareness and use of direct-to-consumer personal genomic tests from four state population-based surveys, and implications for clinical and public health practice. Genet. Med. $14,860-867$.

27 Langford, A.T., Resnicow, K., Roberts, J.S., 2012. Racial and ethnic differences in direct-toconsumer genetic test awareness in HINTS2007: socio-demographic and numeracy correlates.

J. Genet. Couns. 21, 440-447.

28 McGowan, M.L., Fishman, J.R., Lambrix, M.A., 2010. Personal genomics and individual identities: motivations and moral imperatives of early users. New Genet. Soc. 29, 261-290.

29 McGowan, M.L., Fishman, J.R., Settersten Jr., R.A., Lambrix, M.A., Juengst, E.T., 2014. Gatekeepers or intermediaries? The role of clinicians in commercial genomic testing. PLoS ONE 9 (9), e108484. http://dx.doi.org/10.1371/journal.pone.0108484.

30 McGuire, A.L., Burke, W., 2008. An unwelcome side effect of direct-to-consumer personal genome testing: raiding the medical commons. JAMA 300, 2669-2671.

31 McGuire, A.L., Diaz, C.M., Wang, T., Hilsenbeck, S.G., 2009. Social networkers' attitudes toward direct-to-consumer personal genome testing. Am. J. Bioeth. 9, 3-10.

32 Meisel, S.F., Carere, D.A., Wardle, J., Kalia, S.S., Moreno, T.A., Mountain, J.L., Roberts, J.S., Green, R.C., PGen Study Group, 2015. Explaining, not just predicting, drives interest in personal genomics. Genome Med. 7 (1), 74. http://dx.doi.org/10.1186/s13073-015-0188-5 (Aug 1).

33 Merz, J.F., Magnus, D., Cho, M.K., Caplan, A.L., 2002. Protecting subjects' interest in genetic research. Am. J. Hum. Genet. 5, 35-42.

34 Oliver, J.M., Slashinski, M.J., Wang, T., Kelly, P.A., Hilsenbeck, S.G., McGuire, A.L., 2012. Balancing the risks and benefits of genomic data sharing: genome research participants' perspectives. Public Health Genomics 15, 106-114.

35 Ortiz, A.P., López, M., Flores, L.T., Soto-Salgado, M., Finney Rutten, L.J., Serrano-Rodriguez, R.A., Hesse, B.W., Tortolero-Luna, G., 2011. Awareness of direct-to-consumer genetic tests and use of genetic tests among Puerto Rican adults. Prev. Chronic Dis. 8, A110.

36 Ostergren, J.E., Gornick, M.C., Carere, D.A., Kalia, S.S., Uhlmann, W.R., Ruffin, M.T., Mountain, J.L., Green, R.C., Roberts, J.S., 2015. How well do customers of direct-to-consumer personal genomic testing services comprehend genetic test results? Findings from the impact of personal genomics study. Public Health Genomics 18 (4), 216-224. http://dx.doi.org/10.1159/000431250.

37 Powell, K.P., Christianson, C.A., Cogswell, W.A., Dave, G., Verma, A., Eubanks, S., et al., 2012. Educational needs of primary care physicians regarding direct-to-consumer genetic testing. J. Genet. Couns. 21 (3), 469-478. 


\section{Chapter 1}

38 Rahm, A.K., Feigelson, H.S., Wagner, N., Le, A.Q., Halterman, E., Cornish, N., Dearing, J.W., 2012. Perception of direct-to-consumer genetic testing and direct-to-consumer advertising of genetic tests among members of a large managed care organization. J. Genet. Couns. 21, 448456.

39 Roberts, J.S., Ostergren, J., 2013. Direct-to-consumer genetic testing and personal genomics services: a review of recent empirical studies. Curr. Genet. Med. Rep. 1, 182-200.

40 Rose, A.L., Peters, N., Shea, J.A., Armstrong, K., 2005. Attitudes and misconceptions about predictive genetic testing for cancer risk. Community Genet. 8, 145-151.

41 Schelling, H.R., Seifert, A., 2010. Internet-Nutzung im Alter. Gründe der (Nicht-) Nutzung von Informations- und Kommunikationstechnologien (IKT) durch Menschen ab 65 Jahren in der Schweiz. Zurich Open Repository and Archive, University of Zurich.

42 Schmidlen, T.J., Scheinfeldt, L., Zhaoyang, R., Kasper, R., Sweet, K., Gordon, E.S., Keller, M., Stack, C., Gharani, N., Daly, M.B., Jarvis, J., Christman, M.F., 2015. Genetic knowledge among participants in the Coriell Personalized Medicine Collaborative. J. Genet. Couns. Aug 27.

43 Smith, J., Freund, A., 2002. The dynamics of possible selves in very old age. J. Gerontol. B Psychol. Sci. Soc. Sci. 57B, 492-500.

44 Tan, Q., Christiansen, L., Bathum, L., Li, L., Kruse, T.A., Christensen, K., 2006. Genetic association analysis of human longevity in cohort studies of elderly subjects: an example of the PON1 gene in the Danish 1905 birth cohort. Genetics 172, 1821-1828.

45 van den Akker, E.B., Deelen, J., Slagboom, P.E., Beekman, M., 2015. Exome and whole genome sequencing in aging and longevity. Adv. Exp. Med. Biol. 847, 127-139. http:// dx.doi.org/10.1007/978-1-4939-2404-2_6.

46 Van El, C.G., Cornel, M.C., Borry, P., Hastings, R.J., Fellmann, F., Hodgson, S.V., Howard, H.C., Cambon-Thomsen, A., Knoppers, B.M., Meijers-Heijboer, H., Scheffer, H., Tranebjaerg, L., Dondorp, W., de Wert, G., 2013. Whole-genome sequencing in health care. On behalf of the ESHG Public and Professional Policy Committee. Eur. J. Hum. Genet. 21, 580-584.

47 Vayena, E., 2014. Direct to consumer genomics on the scales of autonomy. J. Med. Ethics http://dx.doi.org/10.1136/medethics-2014-102026.

48 Vayena, E., Prainsack, B., 2013. The challenge of personal genomics in Germany. Nat. Biotechnol. 31, 16-17.

49 Vayena, E., Gourna, E., Streuli, J., Hafen, E., Prainsack, B., 2012. Experiences of early users of direct-to-consumer genomics in Switzerland: an exploratory study. Public Health Genomics 15, 352-362.

50 Vayena, E., Ineichen, C., Stoupka, E., Hafen, E., 2014a. Playing a part in research? University students' attitudes to direct-to-consumer genomics. Public Health Genomics 17, 158-168.

51 Vernez, S.L., Salari, K., Ormond, K.E., Lee, S.S., 2013. Personal genome testing in medical education: student experiences with genotyping in the classroom. Genome Med. 19, 24.

52 Wasson, K., Sanders, T.N., Hogan, N.S., Cherny, S., Helzlsouer, K.J., 2013. Primary care patients' views and decisions about, experience of and reactions to direct to consumer genetic testing: a longitudinal study. J. Community Genet. 4, 495-505.

53 Wilde, A., Meiser, B., Mitchell, P.B., Hadzi-Pavlovic, D., Schofield, P.R., 2011. Community interest in predictive genetic testing for susceptibility to major depressive disorder in a large national sample. Psychol. Med. 41, 1605-1613.

54 Wurm, S., Lampert, T., Menning, S., Alter, Gesundheit und Krankheit im, 2009. Subjektive Gesundheit. Robert-Koch Institute, Berlin, pp. 79-91.

Other sources:

55 23andme, 2015. Power of one million 18 June Accessed https://blog.23andme.com/news/onein-a-million/. 


\section{Part 2}

Integrating Big Data and ICT Solutions

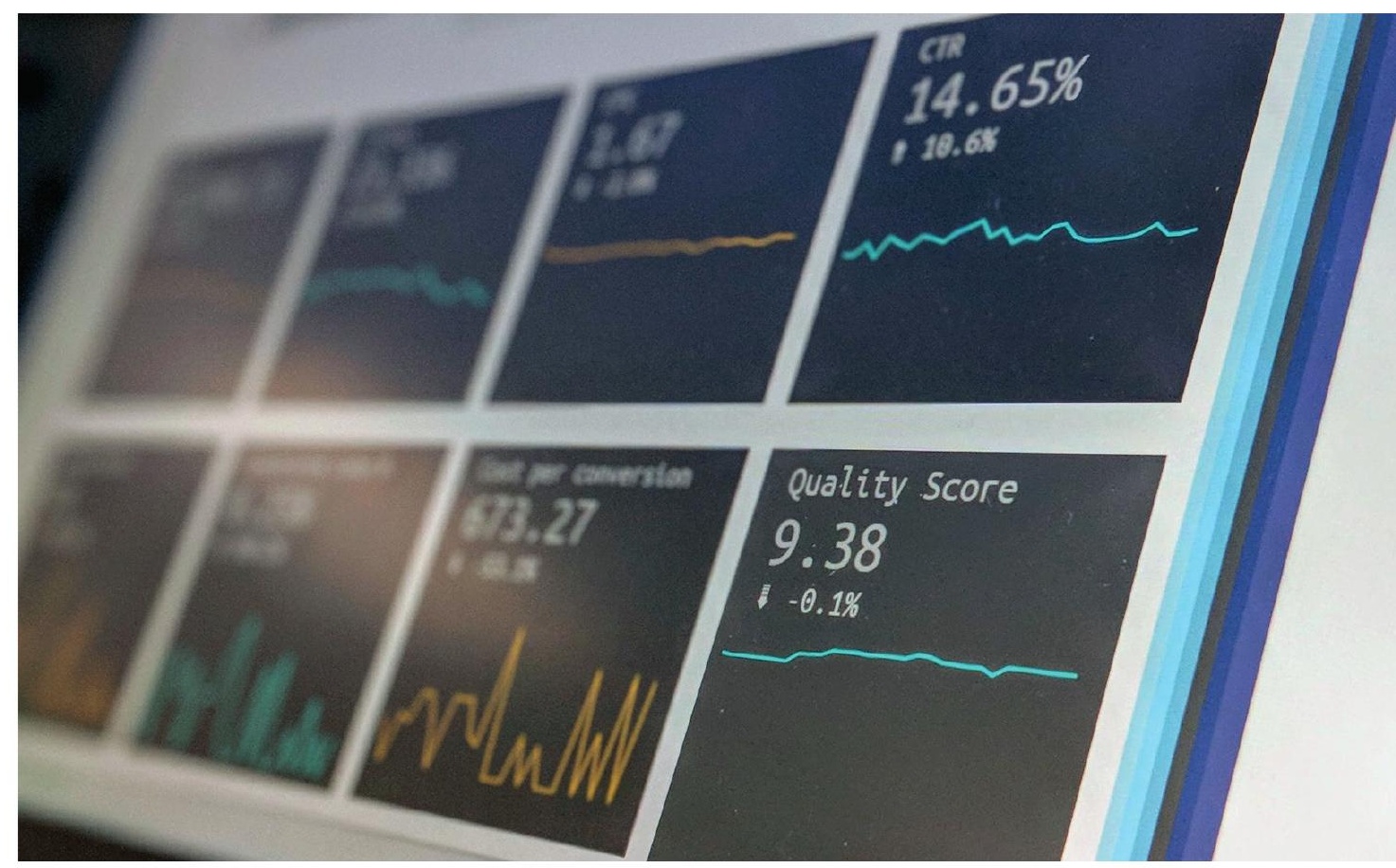


Part 2 
The development of PM will rely heavily on integrated 'Big Data' analytics and ICT solutions to generate the required knowledge and infrastructure to support the new approaches. Technologies for data capture and management and development of high quality databases will be instrumental, but there will also be a requirement for strategies to make sense of this Big Data for known and future purposes. [...] To integrate all these aspects, further European Big Data and 'big science' frameworks need to be created and supported by suitable legislation.

SRIA Challenge 2, [6]

Digital technologies are changing the way people are doing things. The collection, storage, processing and analysis of large amounts of heterogeneous data at high speed is a defining characteristic of today's societies. It is apparent that "Big Data", defined by the seven V's including volume (growing amount), velocity (faster, sometimes real-time collection), variety (of shape and structure), variability (heterogeneous data sources) veracity (reliability) and value [92] will play an increasingly important role in healthcare and health policy making. Traditionally, data have been collected in silos and for a specific purpose. Advances in digital technologies, however, are transforming healthcare with seemingly unlimited opportunities to meet the challenge of improving health and providing better, safer and more sustainable care for all [93]. While developments in multiple sectors have had a revolutionary impact, the same cannot be said for the use of technology and data to improve health care policy and services. The development of PM will rely heavily on integrated Big Data analytics and ICT solutions to generate the required knowledge and infrastructure to support the new approaches. Furthermore, data-driven decision making in policy or the clinical context will enable the realization of personalization of medicine.

Objective (2): Investigate the potential of Big Data as a support tool for making innovative decisions in public health policy making.

The second objective has been addressed by one peer reviewed scientific publication by Mählmann et al. [94] entitled "Big Data for Public Health Policy Making: Policy Empowerment" (Chapter 2). It aims to give an overview of the potential opportunities of Big Data and to propose concrete solutions to integrate Big Data in the health care and policy making context. 
Part 2 


\section{Chapter 2}

\section{Big Data for Public Health Policy-Making: Policy Empowerment}

Published as: Mählmann L, Reumann M, Evangelatos N, Brand A. Big Data for Public Health Policy- Making: Policy Empowerment, Public Health Genomics, doi: 10.1159/000486587 [IF:1.64] 


\section{Chapter 2}

\section{Abstract}

Digitization is regarded to radically transform healthcare. As such, with seemingly unlimited opportunities to collect data, it will play an important role in the public health policy-making process. In this context, health data cooperatives (HDC) are a key component and core element for Public Health policy-making and for tapping the potential of all existing and rapidly emerging data sources. Being able to leverage all the data requires overcoming computational, algorithmic and technological challenges that characterize today's highly heterogeneous data landscape, as well as a host of diverse regulatory, normative, governance and policy constraints. The full potential of Big Data can only be realised if data is being made accessible and shared. Treating research data as public goods, creating health data cooperatives to empower the citizens through citizen-owned health data, and enabling data access for research and development of new diagnostics, therapies and public health policies will yield the transformative impact of digital health. The Health Data Cooperative model for data governance is an arrangement, based on moral codes, that fosters citizens to participate in the improvement of their own health. This then enables Public Health and the policy maker to evaluate policy changes and evaluate the impact and risk of population level. 


\section{Introduction}

History has shown great improvements in the practice of public health policymaking. In the mid-19th century, the case of the Broad Street cholera outbreak in London and the painstaking work on identifying the Broad Street water pump as the source of the outbreak is a great example of the reactive way of public health practices and policy-making in their early days [1]. Nowadays, in the midst of a number of profound societal changes including the dynamics of populations' age structure, technological developments, and the burden of chronic diseases, public health policy-making aims to apply a more proactive approach to healthcare by establishing health-promoting and preventive activities. However, total expenditures tend to rise much faster in healthcare than in non-health areas, and in a potentially unsustainable way [2].

Besides being a value in itself, health is a precondition for economic prosperity, and hence efficient spending on health can promote economic growth. Consequently, citizens' health has become a core value of the European Union (EU) (Art.168, TFEU) [3]. On the other hand, public health policy-making is defined as any kind of decision, plan, and action undertaken to achieve specific healthcare goals within society. Therefore, it aims to define visions for the future and, in turn, helps to establish targets and points of reference and outlines priorities in order to protect health as a value [4]. Accordingly, the EU public health policy generates economies of scale by pooling resources to tackle common challenges. Furthermore, it complements national policies and encourages cooperation and coordination across countries to reach sustainable health systems, health programs, and health coverage for all and to overcome social exclusion [5].

In particular, according to the European Commission [3] current public health policy challenges include (1) the need for evidence-based policy-making, (2) the necessity to increase cost-effectiveness and become more prevention oriented, (3) the early detection of outbreaks of new threats and resistances, (4) the development of preventive strategies targeting risk factors for chronic diseases, (5) the need to move from a "one-size-fits-all" approach in medicine towards more personalized healthcare, and (6) the improvement of cross-border care, especially for rare diseases.

\section{Status Quo}

The current driver of change in all industries is digital transformation, and through digital transformation the collection, storage, processing, and analysis of large amounts of heterogeneous data at high speed: big data. Massive amounts of data generated from an increasingly diverse plurality of sources offer opportunities for 


\section{Chapter 2}

new insights into previously unknown fields. Various terms associated with the collection, analysis, and application of big data in health are floating around in the field of healthcare, including e-health, m-health, digital health, health information technology, health 2.0, e-medicine, telemedicine, etc. [6]. This variety of terms is surpassed only by the variety of data sources that can be combined to improve health, such as "-omics" data, imaging data, medical test results, publications, guidelines, and text books. New data sources are also emerging: lifestyle data from social media, exogenous data, and the Quantified Self movement, but also environmental and demographic data can impact public health decision- and policy-making through, e.g., epidemiological modeling of disease outbreaks to come up with the best action that should be taken. In addition, mobile health apps are flooding the personalized healthcare ecosystem, adding to the wealth of data sources that can be tapped into in order to improve the health of society through public health policies and measures. Finally, new research and clinical applications allow generating larger cohorts and populations from which high-resolution data and high-quality biological samples can be obtained. These can be used as further data sources. These data - stored in registries and biobank databases, for example - are a very valuable resource for public health policies in the present and in the future. Both individual-level and aggregated data can facilitate the investigation of health outcomes and disease courses of individuals as a foundation for improved personal health and care [7].

The private sector is rapidly adopting data strategies for decision-making, while governments are embracing the latest tools and technologies more slowly [8]. Most governmental organizations are not prepared to apply big data analytics to their strategic benefit, that is, to optimize their way of working, to redefine their role or actions, to consider the disruptive nature of technological changes, and to investigate the topic at large and find benefits for policy development, definition, and evaluation [8]. For governmental actions, technology is still regarded as a complementary tool to the existing organizational and administrative practices, but it is hardly viewed as capable of triggering changes to the actual structures of decision-making processes [9]. An important reason for the slow process of big data adaption in healthcare lies in the nature of big data: many players, providers, and facilities are unable to share data, not even within one organization, due to concerns and regulations pertaining to privacy and data protection.

Next to their strategic potential, big data can be seen as a societal, economic, and political asset, giving those who can access and use it a significant competitive advantage. Big data technologies and services are expected to grow worldwide from USD 16.9 billion in 2015 at a compound annual growth rate of $40 \%-$ about 7 times that of the information and communications technology market overall. According to the prognosis of demosEUROPA, big data have the potential to increase the European GDP by $1.9 \%$ by 2020 [10]. In the health and social 
care sector alone, the McKinsey Global Institute estimated the "big data value" at EUR 90 billion, considering only the reduction in national healthcare expenditure in the EU [11]. From the above it is obvious that, apart from its benefits for health, digital transformation in healthcare is becoming increasingly critical for economic growth and sustainability as well.

The rise of new technologies for data collection, data sharing practices, and computing power - in sum, big data - provide an opportunity to gain insights from new and emerging types of data and content and to answer questions that have previously been considered beyond our reach to answer. At the same time, public health policy-making aims to effectively estimate and evaluate risks [12], to meaningfully allocate scarce resources, and to anticipate possible unintended consequences. The key to successful and effective public health policy-making is, therefore, to rely on relevant and timely evidence to support the development of participatory interventions.

The urgent need to improve individual and population health and to move from reactive or proactive towards predictive or even prescriptive decision-making, coupled with the seemingly unlimited enthusiasm regarding the potential benefits of big data, leads us to fundamental questions: how can we integrate big data into public health policies and how can we empower public health policy-making through the application of big data?

In order to answer these questions, we first describe the challenges and opportunities of big data for public health policy-making. We then discuss the concept of health data cooperatives (HDC) as an essential part of a new data framework that could allow the effective implementation of big data in public health policy-making.

\section{Challenges to Big Data Integration}

To reach the goal of exploiting the potential of these existing and rapidly emerging data sources requires overcoming several challenges that characterize today's highly heterogeneous data landscape, as well as a host of diverse regulatory, normative governance, and policy constraints. As a result, not only technical and infrastructural challenges but also ethical, legal, and societal ones must be addressed $[13,14]$.

The first overarching challenge is the integration of big data into public health policies in order to seize the opportunities of the new data economy to produce insights for public health and thereby prevent falling behind other world regions and sectors.

The second challenge is that in most EU Member States, the data that could be useful for the evaluation of health interventions and health trajectory 


\section{Chapter 2}

predictions are not interlinked. This greatly reduces the value of these data as a basis for public health decision-making. The lock-in effect of such data silos is currently the biggest obstacle to the integration of data sets into European healthcare systems. Record linkage is, however, a prerequisite for effective big data analytics for public health policies.

The third challenge is realizing the potential of new data sources, such as data produced by wearable devices and mobile apps, for public health knowledge and policy. So far, this has proven very difficult for both technical (different data formats) and social reasons (this kind of data is generated outside the traditional settings of public health [surveillance], biomedical research, or clinical medicine).

The fourth challenge is developing a strong platform on which heterogeneous cross-border data sets can be collected, as well as a strong analysis interface that will allow for (near-) real-time data analysis and provision of "readable and usable" information to policymakers.

The fifth challenge is finding the right balance between the goals of contributing data for public health purposes and concerns about personal privacy. New opportunities for linking and integrating discrete data sets present challenges to privacy, and therefore require highly trustworthy institutions and good governance solutions.

The EU has funded the collection of data in registries, patient cohorts, and biobanks in the past, and these projects have generated considerable value for research, policy, and society more widely [15]. However, the full potential of these data sources has not been unlocked, and their value can be increased if more data sources are linked and analyzed in context. To overcome these challenges and to reach the ambitious goal beyond health data linkage, the right data framework is needed to better acquire, manage, share, model, process, and exploit the huge amount of data. Furthermore, such a framework will help to develop integrated solutions that support public health authorities in healthcare system management, long-term policy-making, and the ability to provide actionable insights at the point of care. The full potential of big data can only be realized if a new trust model and framework is implemented that puts citizens/ patients at the center and enables them to assume a proactive role in managing their healthcare [16].

\section{HDC for Policy Empowerment}

To overcome the challenges of integrating big data into policy-making, we propose HDC as the needed framework for policy empowerment. The rapid technological advances in genome sequencing and mobile health monitoring of various lifestyle parameters, paired with the decreasing costs of data storage and 
computing power and the increased security capabilities of cloud-based storage and analysis systems [17], make the formation of HDC possible and timely. HDC are unified data systems that promote data access to and data linkage of heterogeneous data from a multitude of sources within and outside a health domain. HDC offer a framework for overcoming technical, societal, and political challenges and to empower policy-making through the integration and application of big data. HDC are primarily based on metadata and have an inherently citizen/patient-centered trust model structure for health data governance. This way, they allow for the integration of heterogeneous citizen health data, as well as linkage to knowledge published in articles, guidelines, and databases, thereby making it possible to create value and insight through an analytics platform that can sit on top of any data governance model [18]. Hence, the HDC model addresses all the challenges mentioned above and offers a comprehensive solution in a single framework.

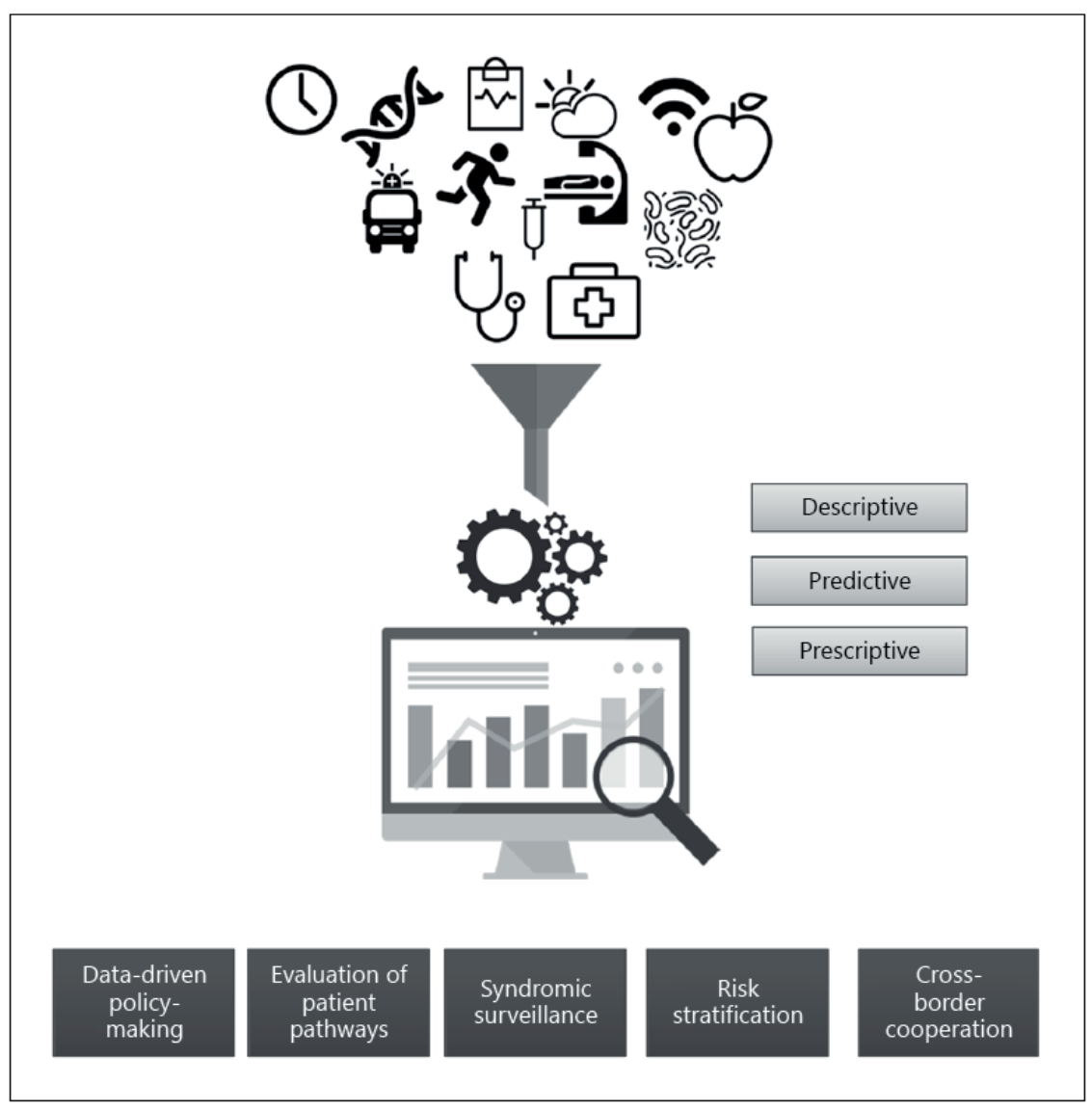

Figure 1: Health data cooperative framework. 


\section{Chapter 2}

As illustrated in Figure 1, an HDC is an ecosystem where stakeholders' data, knowledge, materials, and services are integrated into a cloud-based framework. At first, its heterogeneous components might include data sources such as national electronic patient record and nonelectronic systems, research data, repositories of biological material, genomic and proteomic data, citizen and patient $\mathrm{m}$ health data, sensor data, environmental data, biostatistics, social media data, and more. This diversity of data sources is processed, analyzed, and mapped comprehensively in order to create a network of knowledge and actionable insights. Analytical models will be constantly redefined, new requirements will be captured, and new sources of data acquisition will be identified. The aims of analysis are to obtain descriptive analytics to study causal mechanisms for the current state, predictive analytics to improve prognoses on the spatial and temporal development of ill-health and disease, and, if possible, prescriptive analytics to inform policy-making on how to decide on shaping the future [19]. The distributed nature of big data requires substantial investments in technical solutions. For example, computational resources have to be available to make the analysis of whole data sets feasible. But technology is not the bottleneck, given the respective computational resources. In the case of genomic data, for example, high-performance computing techniques, with massive use of parallel distributed memory supercomputers, can overcome computational challenges for an exhaustive analysis of whole data sets [20]. Based on this, a multitude of potential applications can be developed to support policy-making.

Additional potential issues of HDC that should be considered are that data should remain in citizen ownership and control. This way, citizens might benefit from data analysis, visualization, and interpretation tools. In the same way, blood banks and citizen science platforms work best when no financial incentives are offered [21]. HDC should remain not-for-profit, and potential benefits should be directly reinvested in platform services and research projects for the benefit of society and serving the common good [22]. Furthermore, all data processing should be executed in a transparent and secure way. The data should be multiply encrypted and data access be logged. For example, the HDC model allows the construction of regional/national cooperatives which, by a set of common rules, permit global (research) projects to be set up and carried out in a fair and decentralized manner. Finally, the framework should be based on trust: transparency, information, and openness are key instruments to obtain this. Transparency goes far beyond informing citizens of the "terms of conditions" published on a website. Striving for transparency means making processes, contents, and decisions intelligible and accessible, as well as fostering education, skill development, and discussion among all stakeholders. Similarly, ethics and transparency go beyond simple legal compliance; that is, data governance must allow data providers and adequate democratic governance bodies - to constantly monitor data usage 
as well as checks and balances including rigorous ethics oversight. This kind of rigorous and ethical oversight should cover the algorithms and tools as well, since transparency is needed to make sure that the algorithms do what they are supposed to do. An integral part is the trust model, which, hosted in a secure, privacyprotecting cloud environment, empowers citizens to govern their data while at the same time giving public health policymakers the opportunity to build an unprecedented health ecosystem that allows public health professionals, the private sector, policymakers, citizens, and other stakeholders in the health system and beyond (Health in All Policies) to harness big data insights across the spectrum of healthrelated data.

In the following paragraphs, a series of examples are presented that illustrate the potential of HDC for tackling the abovementioned public health policy challenges.

First, to realize the challenge of evidence-based policy-making, large amounts of data have to be turned into actionable information to authorities for planning and implementing public health activities. With HDC, policy-making is not only evidence based but also data driven. A revolution in government decision-making is waiting to be launched. Inspired by the examples of New York's predictive policing activities or Obama's data-driven campaigning, also health prevention strategies - such as, for example, for breast cancer screening - have the potential to become more targeted if based on an increased number of consulted data sources. This way, detection rates will increase, the burden of false-positive results will decrease, and cost-effectiveness will be realized.

Second, in order for the healthcare systems to become more cost-effective and prevention oriented, a potential application of HDC might be the evaluation of patient pathway management both at the primary care level (prevention and early detection) and en route. Big data insights open the door to evaluating past policies on prevention strategies and disease screening. Citizens and patients should be engaged in the process and be invited to give their feedback to prevention and screening and the reasons for uptake or refusal. This can be repeated at different points in time in order to systematically monitor changes in attitudes and understandings. For example, the Hungarian government developed the mobile health application MENTA to monitor patient pathways and evaluate health programs. This way, MENTA integrates personal attitudes with relevant background data. In addition, tracking social media can assist policymakers in understanding how people experience a certain policy and drive their decisions for more responsive and resilient policies [9]. What is now realized with MENTA should be available on a broader scale via HDC, and information obtained via social media should be fed into the HDC system. 


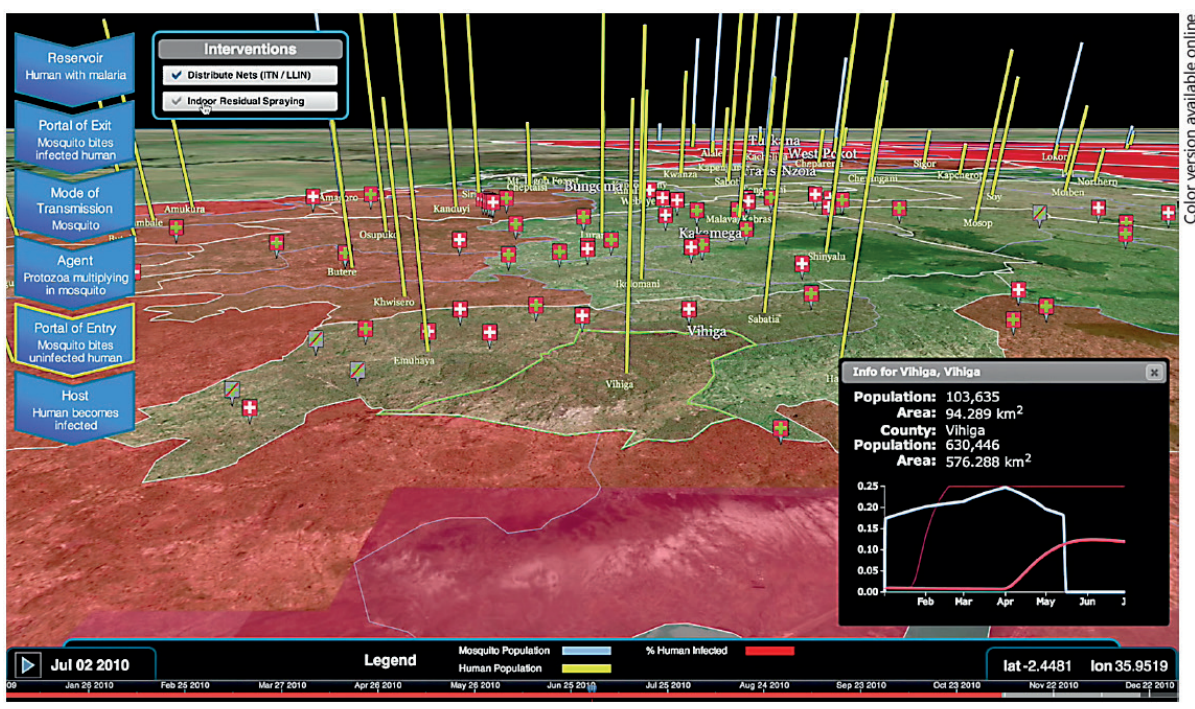

Figure 2: The IBM Cognitive Health Care and Health Systems Hub demonstration allows users to interact with the chain of causation for malaria in Kenya to simulate the impact of public health measures.

Third, regarding early detection and epidemiological challenges, HDC allow the alignment of big data and advanced simulation methods in order to provide highleverage policy analyses to public health officials across a range of epidemiological challenges. Specifically, HDC could be applied as a tool for syndromic surveillance. Syndromic surveillance has proven its effectiveness in public health policy-making as a means of overcoming information gaps and supporting the process of policy-making for health promotion, health protection, and health services [23]. As defined by the EU Triple-S initiative [24], Syndromic Surveillance is the real-time (or near real-time) collection, analysis, interpretation and dissemination of healthrelated [big] data to enable the early identification of the impact (or absence of impact) of potential human or veterinary public health threats which require effective public health action. Syndromic surveillance is based not on the laboratory confirmed diagnosis of a disease but on (heterogeneous) nonspecific health indicators including clinical signs, symptoms as well as proxy measures (e.g. absenteeism, drug sales, production collapse in animal health) that constitute a provisional diagnosis (or 'syndrome'). The data are usually collected for purposes other than surveillance and, where possible, are automatically generated so as not to impose an additional burden on the data providers. This surveillance tends to be non-specific yet sensitive and rapid, and can augment and complement the information provided by traditional test-based surveillance systems. 
Furthermore, a comparison with previous year's seasonal data could trigger warning messages in the case of extreme deviations or repetition of disease outbreaks. Since these data report symptoms, they reflect a point in time close to the onset of the actual disease. Such a system could be easily adopted in a multitude of public health affairs, and even an automatic surveillance system could be programmed to inform about deviations. This way, an HDC surveillance system would provide the responsible authorities with optimal data which they can explore and interact with as a support tool for decision-making and policy information. Additional features should include the geographical spread of a disease, as well as a time evolution graph. A demonstration was given by Davis et al. [19], where epidemiological modeling of malaria, population, weather, and geographical data from Kenya was used to simulate public health policy measures such as the impact of handing out mosquito nets versus indoor residual spraying on infection rates (Fig. 2). Users could interact with the data and simulation for decision support and policy information. Fourth, to improve the prevention of chronic diseases, data linkage in HDC can lead to evidence-based prevention strategies in order to evaluate the efficiency and effectiveness of implemented strategies, to give feedback on results, and to support the development of methods. Temporal patterns of risk have been analyzed with the focus mainly on the progression of specific diseases and less on the general problem of risk estimation covering the full spectrum of diseases and their outcomes [25]. Other large-scale approaches have addressed disease risk and outcomes without considering the time element. Large-scale data analyses focusing on defining a comprehensive set of disease outcome trajectories among millions of citizens have to date been performed based on registry data for a specific purpose. However, a key prerequisite for risk-based preventive interventions is individual risk estimation. This is where HDC provide an advantage, as they cover a real-time ("live") comprehensive view of the current state of all citizens. A discovery-driven analysis of spatial and temporal disease progression patterns using data from electronic health registries of whole populations could be applied to permit real-time analyses of risk trajectories at the individual level. Data across an HDC population need to be grouped into risk patterns, which then allows an individual trajectory to be compared with these patterns.

Fifth, to overcome the current practice of "one-size-fits-all" treatment, HDC can leverage information for the development of innovative approaches to improve current risk stratification methodologies and adjust therapies and prevention strategies to the individual's needs, opening the way for personalized medicine [26]. The first success stories in this context are mainly reported in the field of oncology [27]. Targeted testing for specific genetic variations has transformed disease classification and treatment. However, the development and implementation of personalized approaches to other diseases and many aspects of 


\section{Chapter 2}

healthcare delivery are still far from being a reality [28]. The HDC infrastructure permits knowledge creation based on, for example, diagnostic images, genetic test results, and biometric information from electronic health records in combination with sensor data and other patient characteristics, thereby yielding better and more personalized decision-making and outcome assessment.

Sixth, an urgent problem in cross-border areas is the challenge of overcoming fragmentation and providing optimal care for rare diseases. HDC offer a solution in delivering cross-border and networking coordination and technology integration to facilitate interoperability between the components of the big data value chain. An HDC platform could serve as a basis to facilitate cross-border cooperation and data sharing for healthcare purposes. This task will prove how the actual challenges faced by national actors about to implement the cross-border directive can be overcome. Furthermore, also in the context of rare diseases, HDC will allow data sharing and access anywhere in the world across all borders. Due to the inherent trust model and consent life cycle management, patients can share their information with any stakeholder in the health system, which allows more collaboration and higher efficiency. This leads to better and faster means for the correct diagnosis of a rare disease - for example, in cases in which medical experts and treatment means are in different geolocations.

With the use of HDC, measures can be tracked which are likely to be influenced by policy innovation in a matter of weeks rather than years, allowing for a substantially faster research cycle than with traditional methods. This framework provides timely insights within weeks or months, with results reflecting current clinical practice, which constitutes a substantial improvement over the traditional research cycle. Data are drawn from a large international sample rather than from a single center or a single state. HDC reduce the burden of collecting and managing raw data for analysis.

\section{First Step: Paradigm Shift}

A paradigm shift towards the application of big data is essential for public health policy-making. Furthermore, the proposed solution of HDC might enable us to reach multiple goals at the same time: data driven policy-making and the realization of personalized policy-making. However, certain requirements need to be addressed: how can big data be utilized for the common good whilst respecting individuals' rights and liberties, such as the right to privacy? Therefore, transparency, information, and openness are key instruments to establish trust. Transparency goes far beyond informing citizens of the "terms and conditions" published on a website. Striving for transparency means making processes, contents, and decisions intelligible and accessible, as well as fostering education, skill 
development, and discussion among all stakeholders. Similarly, ethics and transparency go beyond legal compliance only through an ethical layer, where data and algorithm providers and democratic governance bodies monitor the use of data, with checks and balances including rigorous ethics oversight. Besides the many opportunities that big data provide for policy-making in public health, they also pose many challenges at a regional, national, and European level [28]. As data used for health purposes regularly come from other domains, one has to take the Health in All Policies approach into account. New multilevel accountabilities have to be analyzed, since the information underlying big data will fall under different (legal) responsibilities with different forms of access. "Open government" strategies differ widely in Europe. Furthermore, public health surveillance and public health research are governed by national and international legislation and guidelines. However, many of these norms were developed in response to very different historical conditions, including technologies that have now been superseded $[29,30]$. Thus, the availability of, access to, and use of relevant data will be different too. As, for instance, environmental threats do not stop at borders, relevant existing national and European regulations from other policy areas have to be analyzed as to their usability in a cross-border health setting.

\section{Conclusions}

This paper discussed the potential use of big data as a tool for empowering public health policy-making. The full potential of big data can only be realized if data are made accessible and shared. Treating research data as a public good, creating HDC to empower citizens through ownership of their health data, and allowing data access for research and the development of new diagnostics, therapies, and public health policies will show the transformative impact of digital health. The HDC model of data governance has an inherent trust structure that fosters citizens' participation in their own health care. This then enables public health institutions and policymakers to evaluate policy changes and assess impacts and risks at the population level.

In this context, HDC should be a key component of public health policy-making, exploiting the potential of any existing and rapidly emerging data sources. Being able to leverage all the data requires overcoming the computational, algorithmic, and technological challenges that characterize today's highly heterogeneous data landscape, as well as removing a host of diverse societal, cultural, regulatory, normative, governance, and policy constraints. 
Chapter 2

\section{Acknowledgements}

We thank Sebastian Schee genannt Halfmann (Maastricht University) for his valuable feedback on the personalized medicine aspects of the paper.

\section{Statement of Ethics}

Ethical standards were followed in the conduct of the study.

\section{Disclosure Statement}

All authors declare no conflict of interests. The entire study was performed without external funding. 


\section{References}

1 Khoury MJ, loannidis JPA: Big data meets public health. Science 2014;346:1054-1055.

2 Weeramanthri TS, Bailie RS: Grand challenges in public health policy. Front Public Health 2015;3:29.

3 European Commission: EU Health Policies 2017. https://ec.aeuropa.eu/health/strategy/ policy_en (cited August 9, 2017).

4 WHO: Health Policy 2017. http://www.who. int/topics/health_policy/en/ (cited August 9, 2017).

5 Kuipers Cavaco Y, Quidbach V: Public Health in the EU: State-of-Play and Key Policy Challenges 2014. http://www.europarl.europa.eu/RegData/etudes/IDAN/2014/536286/IPOL_IDA(2014)53 6286 EN.pdf (cited November 9, 2017).

6 European Commission Report: Study on Big Data in Public Health, Telemedine and Healthcare 2016. https://ec.europa.eu/health/ sites/health/files/ehealth/docs/bigdata_report_en.pdf (cited May 10, 2017).

7 Leyens L, Reumann M, Malats N, Brand A: Use of big data for drug development and for public and personal health and care. Genet Epidemiol 2017;41:51-60.

8 European Commission Report: Big Data Analytics for Policy Making 2016. https://joinup.ec.europa.eu/sites/default/files/document/2016-07/dg_digit_study_big_data_analytics_for_policy_ making.pdf (cited May 10, 2017).

9 Höchtl J, Parycek P, Schöllhammer R: Big data in the policy cycle: policy decision making in the digital era. J Organ Comput Electron Commerce 2016;26:147-169.

10 Buchholtz S, Bukowski M, Śniegocki A: Big and open data in Europe: a growth engine or a missed opportunity. Warsaw Institute for Economic Studies Report Commissioned by demosEUROPA 2014;10.

11 McKinsey Global Institute: Big Data: The Next Frontier for Innovation, Competition and Productivity. 2011. http://www.mckinsey.com/insights/business_technology/big_data_the_nextfrontier_for_innovation (cited May 10, 2017).

12 Meyer CO: Over- and under-reaction to transboundary threats: two sides of a mis- printed coin? J Eur Public Policy 2016;23: 735-752.

13 OECD: Health Data Governance: Privacy, Monitoring and Research - Policy Brief. 2015. https://www.oecd.org/health/health-systems/Health-Data-Governance-Policy-Brief. pdf (cited November 9, 2017).

14 Eisenstein M: Big data: the power of peta-bytes. Nature 2015;527:2-4.

15 CORDIS: Final Report Summary - BIOSHARE-EU (Biobank Standardisation and Harmonisation for Research Excellence in the European Union) 2016. http://cordis. europa.eu/result/rcn/184957_en.html (cited November 9, 2017).

16 Blasimme A, Vayena E: Becoming partners, retaining autonomy: ethical considerations on the development of precision medicine. BMC Med Ethics 2016;17:67.

17 Kuo AM: Opportunities and challenges of cloud computing to improve health care services. J Med Internet Res 2011;13:e67.

18 Hafen E, Kossmann D, Brand A: Health data cooperatives - citizen empowerment. Methods Inf Med 2014;53:82-86.

19 Davis M, von Cavallar S, Wyres KL, Reumann M, Sepulveda M, Rogers P: Intuitive informa- tion and knowledge representation of disease incidence and respective intervention strategies. Stud Health Technol Inform 2014;205: 1173-1177.

20 Goudey B, Abedini M, Hopper JL, Inouye M, Makalic E, Schmidt DF, Wagner J, Zhou Z, Zobel J, Reumann M: High performance computing enabling exhaustive analysis of higher order single nucleotide polymorphism interaction in Genome Wide Association Studies. Health Inf Sci Syst 2015;3(suppl 1 HISA Big Data in Biomedicine and Health- care 2013 Con):S3.

21 Niza C, Tung B, Marteau TM: Incentivizing blood donation: systematic review and meta- analysis to test Titmuss' hypotheses. Health Psychol 2013;32:941-949. 


\section{Chapter 2}

22 Mählmann L, Röcke C, Brand A, Hafen E, Vayena E: Attitudes towards personal genomics among older Swiss adults: an exploratory study. Appl Transl Genom 2016;8:9-15.

23 Nsubuga P, White ME, Thacker SB, Anderson MA, Blount SB, Broome CV, Chiller TM, Es- pitia V, Imtiaz R, Sosin D, Stroup DF, Tauxe RV, Vijayaraghavan M, Trostle M: Chapter 53 Public health surveillance: a tool for targeting and monitoring interventions; in Jamison DT, Braman JG, Measham AR, et al (eds): Disease Control Priorities in Developing Countries, ed 2. Washington, Oxford University Press, 2006.

24 Triple S Project: Assessment of syndromic surveillance in Europe. Lancet 2011;378: 1833-1834.

25 Jensen AB, Moseley PL, Oprea TI, Ellesøe SG, Eriksson R, Schmock H, Jensen PB, Jensen LJ, Brunak S: Temporal disease trajectories condensed from population-wide registry data covering 6.2 million patients. Nat Comm 2014;5:4022.

26 Schee genannt Halfmann S, Evangelatos N, Schröder-Bäck P, Brand A: European healthcare systems readiness to shift from "one-size fits all" to personalized medicine. Pers Med 2017;14:63-74.

27 Snyderman R: Personalized medicine 2014: has healthcare been transformed? Pers Med 2014;11:365-368.

28 PerMed: Shaping Europe's Vision for Personalised Medicine - Strategic Research Innovation Agenda (SRIA) 2015. http://www. permed2020.eu/_media/PerMed_SRIA.pdf (cited November 9, 2017).

29 Prainsack B: Three "H"s for health - the darker side of big data. Bioethica Forum 2015;8: 40-41.

30 Vayena E, Salathé M, Madoff LC, Brownstein JS: Ethical challenges of big data in public health. PLoS Comput Biol 2015;11:e1003904. 


\section{Part 3}

\section{Translating Basic to Clinical Research}

and Beyond 
Part 3 
In order for PM to reach its anticipated impact on human health and wellbeing, translation of discoveries and communication across the continuum of research are required. This starts with the integration of all 'omics' data to generate and implement meaningful interventions, [...] but also environmental and lifestyle interventions to truly bridge all steps of the PM research continuum.

SRIA Challenge 3 [38]

While the initial success stories of PM have mainly come from the field of oncology, it is at the time that chronic complex diseases (CCD) should shift into focus. These complex multifactorial diseases are important to address in the context of personalization strategies, since they will benefit a large proportion of the population and because of its foreseeable impact on reducing health care costs [95].

To illustrate the scenario, the case of Inflammatory Bowel Diseases (IBD) has been selected. IBD is a chronic complex inflammation characterized by multiple pathogenic components that interact with each other [96]. Further, IBD encompasses two major forms of chronic intestinal inflammation: Crohn's disease (CD) and ulcerative colitis (UC). However, beyond this dichotomy, a number of distinct phenotypes exist within these diseases [97].

Overall goal of treatment is to improve clinical symptoms, maintain clinical remission, improve quality of life, induce steroid free remission, support mucosal healing, initiate histological remission, reduce hospitalization, avoid surgery and cut overall costs and circumvent disease and therapy related complications. However, treatment remains ineffective for at least $50 \%$ of patients with IBD and loses efficacy after the first year, resulting in a large proportion insufficiently treated patients with IBD [98]. As a consequence, patients may develop structural damage of the gastrointestinal system and infections that might require surgery or hospitalization [98]. This scenario is especially worrisome for the pediatric cases of IBD. These young patients will have to cope with a severe burden of disease side effects and consequences of ineffective treatment for their entire life.

Therefore, the case of IBD illustrates the need for the application of more individualized ways of treatment based on individual characteristic [98]. To reach this goal, the first step has been to identify clinical markers that predict TNF treatment response. Second, therapeutic drugs should be monitored narrowly in combination with observing inflammation markers, endoscopic outcomes, imaging and other symptoms of active inflammation [99]. Furthermore, a molecular-based disease classification will be increasingly important to understand IBD and provide patients with optimal treatment [99]. As such, IBD constitutes an opportunity for PM, and strategies should be tailored to maximize the success of the current treatment, minimize loss of response to therapy or relapses in the future, and address the risks associated with specific medications for given patients. 
However, in addition to molecular based therapy, doctors may want to consider external factors that can impact a person's condition, including lifestyle factors. As previously reported, many chronic diseases are associated with lifestyle and public health recommendations for lifestyle modification, including smoking, diet and physical activity, which have been widely disseminated for the prevention and treatment of disease. The role of lifestyle factors in the case of IBD have been described as crucial $[95,100]$.

In sum, the need for PM derives from the realization that today's most challenging medical conditions are chronic complex diseases with multiple pathogenic components that interact with each other. Currently, complex diseases cannot properly be controlled and much less cured by modulating single components at sporadic time points in the course of the disease or administering the same treatment to all patients. Lifestyle interventions, namely physical activity, have been tested as promising interventions in various cases of chronic inflammatory diseases, as well as in adult patients with IBD [102]. To further establish the case for lifestyle in treatment of chronic pediatric IBD, Chapters 3 to 6 aim to fill the gaps of knowledge.

Objective (3): To explore the influence of a lifestyle intervention including regular physical activity on inflammation, fitness, depression and sleep among pediatric patients with IBD.

This objective has been assessed in detail within a clinical case-control trial among pediatric IBD patients. The trial has been published in three original publications and one draft by Mählmann et al. [102-105] entitled "Psychological wellbeing and physical activity in children and adolescents with inflammatory bowel disease compared to healthy controls" (Chapter 3), "Impaired objective and subjective sleep in children and adolescents with inflammatory bowel disease compared to healthy controls" (Chapter 4); "Aerobic exercise training in children and adolescents with inflammatory bowel disease: Influence on psychological functioning, sleep and physical performance - An exploratory trial" (Chapter 5) and "Favorable impact of chronic aerobic exercise training on disease symptoms in children and adolescents with inflammatory bowel disease" (Chapter 6). 


\section{Chapter 3}

\section{Psychological wellbeing and physical activity in children and adolescents with inflammatory bowel disease compared to healthy controls}

Published as: MähImann L, Gerber M, Furlano RI, Legeret C, Kalak N, Holsboer-Trachsler E, Brand S. Psychological wellbeing and physical activity in children and adolescents with inflammatory bowel disease compared to healthy controls. BMC Gastroenterol. 2017 Dec 12;17(1):160. doi: 10.1186/s12876-017- 


\section{Chapter 3}

\section{Abstract}

Background: Children and adolescents with inflammatory bowel disease (IBD) report impairments in daily activities, social interactions and coping. Findings regarding psychological functioning are inconsistent, while limited information is available on objectively assessed physical activity (PA). The aims of the present study were therefore to compare anthropometric dimensions, blood values, psychological functioning and PA of children and adolescents with IBD with healthy controls.

Methods: Forty-seven children and adolescents took part in the study. Of these, 23 were diagnosed with IBD (mean age: 13.88 years, $44 \%$ females). The IBD group was divided into a medically well adjusted "remission-group" ( $n=14$; IBD$R E)$ and a group with an "active state" of disease ( $n=8$; IBD-AD). Healthy controls $(n=24 ; \mathrm{HC})$ were age- and gender-matched. Participants' anthropometric data, blood values and objective PA were assessed. Further, participants completed questionnaires covering socio-demographic data and psychological functioning.

Results: Participants with IBD-AD showed higher erythrocyte sedimentation rate (ESR), C-reactive protein (CRP) values, haemoglobin, and leukocyte values. IBD-AD had poorer psychological functioning and lower PA (average steps per day) compared to IBD-RE and HC. No mean differences were found between IBD-RE and HC.

Conclusions: The pattern of results suggests that effective medical treatment of IBD in children and adolescents is associated with favorable physiological parameters, psychological dimensions and PA. Psychological counselling of children and adolescents in an active state of IBD seem to be advised in addition to standard treatment schedules. 


\section{Background}

Inflammatory Bowel Disease (IBD) is a chronic, debilitating illness characterized by cycles of disease activity and quiescence. IBD is subdivided into Crohn's disease (CD), ulcerative colitis (UC) and atypical phenotypes as described by the Porto criteria (PIBD: Pediatric IBD) [1]. Although the ultimate aetiology of IBD remains unclear, three hypotheses are advanced: (1) A dysregulated inflammation emerges due to interactions between the gut luminal content (intestinal microflora) and the mucosa, especially in genetically predisposed hosts [2]. (2) Genetic factors seem to contribute slightly to the disease pathogenesis. (3) Microbial and environmental dimensions have been identified as possible contributing factors [3].

Worldwide it is estimated that $25 \%$ of all patients suffering from IBD are children and adolescents, with increasing incidence rates [4]. In children with IBD stunting of physical growth and delay in pubertal development are observed [5]. Furthermore, patients with active IBD are known to report increased erythrocyte sedimentation rates (ESR) due to inflammation, decreased hemoglobin due to chronic blood loss in the gut, decreased albumin due to leaking gut at reduced intake and increased infection parameters such as leukocytes and C-reactive protein (CRP), when compared to healthy controls. Once the diagnosis is confirmed, the goals of medical therapy are to relieve symptoms, restore growth and bone health, normalize quality of life and psychosocial functioning, prevent complications and minimize the adverse effects of medications [6]. While the efficacy of anti-inflammatory pharmaceuticals are not $100 \%$ and the side effects might be severe, some young IBD patients might be in a symptom-free remission, while others are still in an active disease state. Accordingly, based on the regularly assessed PUCAI (Pediatric ulcerative colitis activity index) scores and pediatric Crohn's disease activity index (CDAl), participants in the present study were split into those with medically optimized treatment (IBD in remission, PUCAI <10; $\mathrm{CDAI}<150)$ the IBD - RE group, and those with not yet medically optimized treatment (active disease state), the IBD - AD group. As a result, our first hypothesis was that disease severity and the accompanying symptoms would be negatively associated with the physical development of children and adolescents with IBD. Specifically, we expected smaller and lighter body shapes and elevated inflammation markers in participants of the IBD-AD group, compared to IBD-RE and healthy controls.

In recent years, a plethora of studies have suggested that the prevalence of reduced health-related quality of life (HRQOL) and psychiatric disorders are significantly greater in young people with IBD than healthy controls [7-9]. More specifically, adolescents with IBD had higher levels of internalizing disorders such as anxiety and depression [7, 9], due to several factors such as unpredictable, 


\section{Chapter 3}

unpleasant, and embarrassing symptoms, complex, demanding treatment regimens, treatment-related side effects, the everlooming threat of exacerbations of the disease, and the need for surgical procedures in severe cases [10]. The rate of depression may be as high as $25 \%$, and spreads among a broad variety of psychological and social difficulties. However, as reviewed by Ross et al. [8], the evidence these conclusions are based on, were inconsistent, included few paediatric samples, and used coarse- grained psychological instruments. As a result, recognition of the importance of mental wellbeing in young IBD patients is lacking in medical practice. If these psychological conditions are left untreated, mental disorders linked to more severe IBD symptoms might emerge, as well as more frequent IBD flares, higher hospitalization rates, increased health seeking behaviour and lower compliance with treatment $[11,12]$. To counter this, in the present study, validated and reliable self-rating instruments were used to assess dimensions such as psychological wellbeing, social support and peer relationships. Consequently, the second aim of the present study was to expand upon existing research by assessing children's and adolescent's psychological functioning with self-rating questionnaires, investigating specific aspects of psychological functioning, along with a depression screener.

As regards physical activity and sports participation, research showed that children and adolescents with IBD participated less in sports activities and were less fit than their counterparts without IBD [13,14]. In the specific case of paediatric IBD, reduced physical exertion during puberty might lead to impaired bone growth, strength and density, resulting in an increased risk for osteoporosis in the long term [10]. However, a large number of studies have proven the beneficial effect of PA for a diversity of health conditions: First, PA mitigates depressive symptoms while bolstering sleep [15] and cardiovascular fitness [16]. Second, sports participation (via clubs and sports associations) has the potential to increase and deepen children's and adolescents' social skills. So far, research on PA in children and adolescents with IBD has largely been based on rough estimates. Therefore, the third aim of the present study was to assess PA behavior in children and adolescents with IBD, both subjectively using an internationally established questionnaire, and objectively with three methods: First, to investigate participants' fitness, we used the Six-Minute-Walking-Test; second, for strength assessments, we applied a hydraulic grip strength test, and third, to generate long-term PA reports, a FitbitFlex® accelerometer was applied.

Taking into account the state-of-the-art regarding physical development, psychological functioning and physical activity among children and adolescents with IBD, the following three hypotheses were formulated: First, following others [5, 17], we expected impaired anthropometric development among children and adolescents suffering from IBD and increased inflammatory blood markers, and particularly among those in an active state of the disease. Second, on the basis of 
previous research [7-9], we expected poorer psychological functioning in children and adolescents with IBD, than in their healthy counterparts, and again, particularly among those in an active state of the disease. Third, previous findings [18] led us to expect lower levels of PA (both subjective and objective) in children and adolescents with IBD than in their healthy counterparts.

\section{Methods}

\section{Procedure}

All participants were invited to the hospital to measure height, weight, waist circumference (measured $4 \mathrm{~cm}$ above the navel using a standard anthropometry tape) compared to reference values established by Taylor et al. [19] and BMI $(\mathrm{kg} / \mathrm{m} 2)$, which was compared to the WHO international growth references [20]. Following these anthropometric measurements, participants' blood values were collected and physical performance was examined; they also filled out a $30 \mathrm{~min}$ questionnaire with questions on psychological functioning and PA behaviour. All data were collected by two trained research assistants and supervised by a medical doctor.

The present study was approved by the local ethical committee (EKNZ: 2014:220), and was conducted in accordance to the ethical principles laid down in Declaration of Helsinki (Trial registration number: NCT02264275).

\section{Sample}

A total of 31 eligible children and adolescents with IBD were approached between April and November 2015 via the University Children's Hospital Basel (UKBB, Basel, Switzerland). Of these, $29(93.5 \%)$ agreed to participate in the study, though five subsequently withdrew from the study because of time constraints, and three withdraw due to acute illness (flu). The final sample consisted of 23 children and adolescents with diagnosed IBD (mean age: 13.88 years, SD $=3.11$, 10 females (43.5\%), 7 UC, $12 \mathrm{CD}$ and 3 UD). According to the regularly assessed PUCAI (Paediatric ulcerative colitis activity index) scores and Paediatric Crohn's disease activity index (clinical scores), eight participants were in an active disease state and 14 in remission at the time of the study [11]. Inclusion criteria were as follows: (1) aged 6 to 20 years; (2) clinically and histologically confirmed diagnosis of IBD; (3) willing and able to take part in the study; (4) able to communicate and to complete questionnaires in German; (5) written informed consent from both the children/adolescents and their parents (or a legal caregiver). Exclusion criteria were: (1) severe physical diseases of the locomotor apparatus; (2) psychiatric disorders such as psychotic disorders, severe affective disorders, eating 


\section{Chapter 3}

disorders, mental retardation, autism spectrum disorder; (3) unable to communicate and to complete questionnaires in German; (4) among female adolescents: pregnancy, breastfeeding, or intention to get pregnant during the study period; (5) refusal to give written informed consent. In parallel, 24 age- and gendermatched controls (mean age: 12.38 years, $S D=3.24$, females $n=15(62.5 \%)$ ) were recruited by word of mouth recommendations. Inclusion criteria and exclusion criteria for healthy controls were the same as for patients with IBD except that they had to be physically healthy as reported from both participants and parents and as known from medical records. All participants were informed about the study aims and the voluntary and confidential basis of their participation. Written informed consent was signed both by participants and by their legal guardians. Characteristics of the participants subdivided into three groups of IBD-AD, IBD-RE and healthy control are represented in Table 1.

Table 1: Sample characteristics

\begin{tabular}{llll}
\hline & $\begin{array}{l}\text { IBD-AD } \\
(n=8)\end{array}$ & $\begin{array}{l}\text { IBD-RE } \\
(n=14)\end{array}$ & $\begin{array}{l}\text { HC } \\
(n=24)\end{array}$ \\
\hline Age in years & $14.69 \pm 3.25$ & $13.23 \pm 2.96$ & $12.38 \pm 3.24$ \\
Female & $4(50)$ & $6(42.9)$ & $15(62.5)$ IBD \\
Ulcerative colitis & $3(37.5)$ & $4(28.6)$ & \\
Crohn's disease & $5(62.5)$ & $7(50)$ & \\
Undefined colitis & $3(21.4)$ & & \\
Time since diagnosis (in years) $3.67 \pm 2.81$ & $4.1 \pm 2.9$ & \\
\hline
\end{tabular}

Notes: $N=46$

$I B D-A D$ IBD in an active state of the disease, IBD-RE IBD in remission, $H C$ Healthy control

\section{Tools}

\section{Laboratory assessment}

Using the finger prick technique, biochemical parameters were assessed involving inflammatory indices such as albumin $[\mathrm{g} / \mathrm{dl}]$, CRP $[\mathrm{g} / \mathrm{dl}]$, hemoglobin $[\mathrm{g} / \mathrm{dl}]$, ESR [mm/h], hematocrit [\%], thrombocyte [g/dl] and leucocyte [g/dl] count.

\section{Assessing psychological functioning}

To assess psychological functioning, participants completed the KIDSCREEN 27 [21]. The questionnaire consists of 27 items covering five domains, physical wellbeing, psychological wellbeing, autonomy and relations with parents, social support and peers, and school environment. Answers were given on 5-point rating scales $(1=$ not at all, $5=$ extremely/always). Higher mean scores reflect better 
functioning in a specific domain. Validity has been verified by Ravens-Sieberer et al. [21] (Cronbach's $\alpha=.91$ ).

To assess symptoms of depression, the Child Depression Screener (ChilD-S) was completed [22]. The ChilD-S is a self-report screening instrument for prepubertal in- and out-patients in paediatric care. It consists of 8 items assessing how participants have felt for the last 2 weeks ("I am happy, I am doing fine, I feel exhausted, I worry a lot, I feel sad, I get upset quickly, I am not in the mood for anything, I often think I did something wrong"). Participants were asked to select from four alternative responses reflecting different levels of depressive symptomatology. The cut off value for clinically considerable depression is $\geq 11$, with higher values indicating more marked depressive symptoms. Validity has been verified by Frühe et al. [22] (Cronbach's $\alpha=.78$ ).

\section{Assessing physical activity}

Subjective assessment. The short form of the IPAQ (IPAQ-S) questionnaire was applied as an internationally approved estimator of level of PA and sedentary behaviour [23]. It provides a comparison of vigorous- and moderate-intensity PA, walking, total PA and time spent sitting on weekdays over the past seven days. PA data are reported in hours and/or minutes per day and days per week. Where minutes of intense activity exceeded 180 per day, a cut-off of 180 min per day was applied as suggested by the guidelines of the IPAQ group. Data were transformed and summed using standardized IPAQ scoring protocols to indicate total metabolic equivalent minutes (MET-minutes) of PA per week. Total MET- minutes per week was calculated using the following formula [23]:

[Walking MET-minutes $/$ week $=3.3$ * walking minutes * walking days]

+ Moderate MET-minutes $/$ week $=4.0$

* moderate-intensity activity minutes * moderate days]

+ Vigorous MET-minutes $/$ week $=8.0$

* vigorous-intensity activity minutes * vigorous-intensity days]

= Total PA MET-minutes $/$ week.

Furthermore, self-reported fitness was assessed on a 5-point Likert scale. Validity of the instrument has been established by Hagstömer et al. [24] Sitting time was reported as the amount of time in hours and/or minutes participants usually spent sitting on a weekday during the past 7 days.

Objective assessments. Grip force. Maximum isometric grip force of the dominant hand was assessed using the hydraulic hand dynamometer. Participants made three attempts. Mean outcomes were compared to reference data [25]. 


\section{Chapter 3}

Functional capacity. The 6-min walking test (6 MWT), a self-paced, submaximal exercise test, was employed [26]; the test is designed to assess functional exercise capacity in patients with chronic diseases. The $6 \mathrm{MWT}$ is well-standardized and is increasingly being utilized in pediatric populations with chronic diseases [27]. Walking distance is accepted as the main outcome measure of the 6 MWT. Heart rate (HR) is continuously recorded during exercise using an elastic chest strap with heart rate sensor.

Daily PA. The Fitbit-Flex® (Fitbit Inc., San Francisco, CA, USA), a small and light wristband accelerometer which uses three-dimensional motion sensing technology to measure movement $24 \mathrm{~h}$ a day, was applied for a continuous assessment of 5 days ( 2 weekend days and 3 weekdays). It has a simple display of five LED lights which indicate the number of steps taken in a day. Fitbit data were recorded using the 1-min epoch setting and downloaded from the user website via the device's USB docking port in a raw data format. Validation studies have been conducted with children and adolescents [28].

\section{Statistical analyses}

Since normality was violated according to Shapiro-Wilk test, the groups IBD-AD, IBD-RE and $\mathrm{HC}$ were compared using the Kruskal-Wallis test with these diagnostic clusters as independent variable and anthropometric measures, psychological assessments and PA data as dependent variable. Due to heterogeneity of age distribution, Spearman's correlations were performed to determine whether significant correlations would be found beyond age and other dimensions. Accordingly, statistical calculations of anthropometric data, grip strength and 6 MWT were controlled for age. Post-hoc tests after Whitney- $U$ for $p$-values were performed to examine differences between the three groups. The nominal level of statistical significance was set at alpha $<.05$. Further, effect sizes were reported as partial eta-squared [n2]) and considered as follows: small (s) $=.01 \leq \eta p 2 \leq$ .059 , medium $(m)=.06 \leq n p \leq .139$, or large $(I)=n p \geq .14)$ [29]. All statistics were performed with SPSS ${ }^{\circledR} 24.0$ (IBM Corporation, Armonk NY, USA) for Windows ${ }^{\circledR}$.

\section{Results}

All descriptive and inferential statistical information is reported in the Tables and not repeated again in the written text. All Tables compare the three groups of IBD-AD, IBD-RE and HC. 


\section{Anthropometric and laboratory findings}

As shown in Table 2, there was a statistically significant mean difference in thrombocytes $(p=.003)$. According to Mann-Whitney-U Post-hoc test, differences between IBD-AD and HC were significant $(p=.003)$. Further, there were no statistically significant mean differences between the three groups in the following dimensions: waist circumference, waist circumference difference from norm, height, weight to height percentage, weight, BMI, BMI z-score, percentile BMI vs age, z-score height vs age, percentile height vs age and the blood values albumin $[\mathrm{g} / \mathrm{l}], \mathrm{CRP}[\mathrm{mg} / \mathrm{l}]$, hemoglobin $[\mathrm{g} / \mathrm{l}]$, ESR $[\mathrm{mm} / \mathrm{h}]$, hematocrit [\%] and leukocytes $\left[\times 10^{\wedge} 9 / /\right]$. Investigating effect sizes, the IBD-AD group scored moderately lower on BMI z-score, percentile BMI vs age, z-score height vs age and percentile height vs age.

Further, Table 2 shows higher C-reactive protein (CRP) values, hemoglobin values, and leukocyte values in the group of IBD-AD vs IBD-RE and HC. More specifically, six (75\%) IBD-AD patients had low albumin levels, as did eight (61.54\%) IBD-RE patients and $12(50 \%) \mathrm{HC}$ (reference $35-53 \mathrm{~g} / \mathrm{L})$. CRP was high in one (12.5\%) IBD-AD and one (7.1\%) IBD-RE patient (reference $<10.0$ $\mathrm{mg} / \mathrm{L})$. Two patients $(25 \%)$ in the IBD-AD group, two IBD-RE $(14.2 \%)$ and one control (4.2\%) were anemic with mean hemoglobin scores of $101.5 \mathrm{~g} / \mathrm{L}, 114 \mathrm{~g} / \mathrm{L}$ and $100 \mathrm{~g} / \mathrm{L}$, respectively (reference $120-160 \mathrm{~g} / \mathrm{L}$ ).

Assessing the erythrocyte sedimentation rate (ERS) one IBD-AD (12.5\%) was slightly above reference, as well as four $(28.6 \%)$ IBD-RE and six $(25 \%) \mathrm{HC}$ (reference $\left.4.1-5.1 \times 10^{\wedge} 12 / L\right)$. One IBD-RE $(7.1 \%)$ patient had results below reference.

Regarding hematocrit outcomes, two (25\%) IBD-AD, four (28.6\%) IBD-RE (mean $34.27 \%$ ) and three (12.5\%) HC had values below reference $(36-46 \%)$. Thrombocytosis was present in two (25\%) IBD-AD patients (reference $150-450$ $\left.\times 10^{\wedge} 9 / /\right)$. Leukocyte counts were high in two (25\%) IBD-AD, three (21.4\%) IBD$\mathrm{RE}$ and one $(4.2 \%) \mathrm{HC}$ compared to reference $\left(4.5-11 \times 10^{\wedge} 9 / \mathrm{l}\right)$. 


\section{Chapter 3}

Table 2: Anthropometrics and Blood values

\begin{tabular}{|c|c|c|c|c|c|c|c|}
\hline & \multirow{2}{*}{$\begin{array}{l}\text { IBD-AD } \\
(N=8) \\
M \pm S D\end{array}$} & \multirow{2}{*}{$\begin{array}{l}\text { IBD-RE } \\
(N=14) \\
M \pm S D\end{array}$} & \multirow{2}{*}{$\begin{array}{l}\mathrm{HC} \\
(N=24) \\
M \pm S D\end{array}$} & \multicolumn{4}{|c|}{ Statistical Analysis } \\
\hline & & & & $H$ & $p$ & пр2 & $\begin{array}{l}\text { Interpretation } \\
\text { of effect size }\end{array}$ \\
\hline $\begin{array}{l}\text { Waist } \\
\text { circumference } \\
(\mathrm{cm})\end{array}$ & $67.63 \pm 9.04$ & $65.65 \pm 7.17$ & $64.48 \pm 8.29$ & 0.384 & 0.823 & 0.01 & $\mathrm{~s}$ \\
\hline $\begin{array}{l}\text { Difference from } \\
\text { norm }\end{array}$ & $-9.81 \pm 6.15$ & $-8.68 \pm 4.95$ & $-8.82 \pm 6.0$ & 0.521 & 0.783 & 0 & s \\
\hline Height $(\mathrm{cm})$ & $153.48 \pm 17.53$ & $152.69 \pm 12.02$ & $152.26 \pm 18.0$ & 0.212 & 0.903 & 0 & s \\
\hline $\begin{array}{l}\text { Weight to height } \\
(\%)\end{array}$ & $44.08 \pm 3.07$ & $42.94 \pm 2.13$ & $42.77 \pm 3.49$ & 1.108 & 0.585 & 0.02 & s \\
\hline Weight (kg) & $45.28 \pm 15.85$ & $42.79 \pm 12.83$ & $44.37 \pm 15.75$ & 0.215 & 0.899 & 0 & s \\
\hline BMI & $18.6 \pm 3.37$ & $17.91 \pm 2.78$ & $18.36 \pm 3.16$ & 0.074 & 0.965 & 0.12 & $\mathrm{~m}$ \\
\hline BMI z-score & $-0.588 \pm 0.79$ & $-0.34 \pm 0.95$ & $-0.19 \pm 0.87$ & 1.368 & 0.511 & 0.1 & $\mathrm{~m}$ \\
\hline $\begin{array}{l}\text { Percentile BMI vs } \\
\text { Age }\end{array}$ & s $31.98 \pm 24.31$ & $39.82 \pm 26.23$ & $43.89 \pm 26.43$ & 1.386 & 0.516 & 0.1 & $\mathrm{~m}$ \\
\hline $\begin{array}{l}\text { z-score Height vs } \\
\text { Age }\end{array}$ & $s-0.911 \pm 1.73$ & $0.02 \pm 0.86$ & $0.19 \pm 1.01$ & 2.234 & 0.334 & 0.1 & $\mathrm{~m}$ \\
\hline $\begin{array}{l}\text { Percentile Height } \\
\text { vs Age }\end{array}$ & t $32.04 \pm 32.99$ & $52.91 \pm 25.71$ & $54.83 \pm 31.53$ & 2.236 & 0.333 & 0.07 & $\mathrm{~m}$ \\
\hline Albumin $[\mathrm{g} / \mathrm{l}]$ & $23 \pm 21.94$ & $19.32 \pm 19.20$ & $20.79 \pm 18.76$ & 0.767 & 0.686 & 0 & s \\
\hline $\mathrm{CRP}[\mathrm{mg} / \mathrm{l}]$ & $3.02 \pm 4.56$ & $3.28 \pm 5.69$ & $0.71 \pm 0.85$ & 4.159 & 0.125 & 0.13 & $\mathrm{~m}$ \\
\hline Hemoglobin [g/l] & $139.25 \pm 8.62$ & $134.92 \pm 16.26$ & $\begin{array}{l}135.86 \pm \\
12.33\end{array}$ & 1.423 & 0.493 & 0.01 & $\mathrm{~s}$ \\
\hline $\mathrm{ESR}[\mathrm{mm} / \mathrm{h}]$ & $4.83 \pm 0.36$ & $4.87 \pm 0.54$ & $4.88 \pm 0.36$ & 0.281 & 0.874 & 0 & s \\
\hline Hematocrit [\%] & $39.4 \pm 2.11$ & $38.09 \pm 3.99$ & $38.75 \pm 3.17$ & 0.359 & 0.837 & 0.02 & $\mathrm{~s}$ \\
\hline $\begin{array}{l}\text { Thrombocyts } \\
{\left[\times 10^{\wedge} 9 / /\right]}\end{array}$ & $323.25 \pm 55.61$ & $302.58 \pm 56.15$ & $\begin{array}{l}271.55 \pm \\
46.54\end{array}$ & 11.211 & 0.003 & 0.13 & $\mathrm{~m}$ \\
\hline $\begin{array}{l}\text { Leukocyten } \\
{\left[\times 10^{\wedge} 9 / /\right]}\end{array}$ & $10.16 \pm 2.01$ & $9.28 \pm 2.83$ & $8.25 \pm 1.29$ & 4.088 & 0.13 & 0.11 & $\mathrm{~m}$ \\
\hline
\end{tabular}

Notes: $N=46$, degrees of freedom always $=2,41, p<.05$ statistically significant; effect sizes: small $(\mathrm{s})=.01>\eta p 2<.059$, medium $(\mathrm{m})=.06>\eta p<.139$, or large $(\mathrm{I})=\eta p \geq .14$

IBD-AD IBD in an active state of the disease, IBD-RE IBD in remission, $H C$ Healthy Control, Waist circumference norm references is Taylor et al. [19], BMI Body Mass Index; CRP C-reactive protein, ESR Erythrocyte Sedimentation Rate

Psychological functioning

As Table 3 shows, there were no statistically significant mean differences between the three groups (psychological wellbeing physical wellbeing, autonomy and parent relations, social support and peers, school experience). Descriptively, IBD-AD had lower scores on the psychological wellbeing (PWB) dimension of the KIDSCREEN-27, while the other two groups did not differ from each other. The school dimension $(\mathrm{SCH})$ showed a medium effect of .063 , reflecting lower scores in the IBD-AD group. 
Results of the depression scale indicated a medium effect size of .092, with the highest depression scores in the IBD-AD group and no differences between IBD-RE and HC.

Table 3 Psychological functioning and depression

\begin{tabular}{|c|c|c|c|c|c|c|c|}
\hline & \multirow{2}{*}{$\begin{array}{l}\text { IBD-AD } \\
(N=8) \\
M \pm S D\end{array}$} & \multirow{2}{*}{$\begin{array}{l}\text { IBD-RE } \\
(N=14) \\
M \pm S D\end{array}$} & \multirow{2}{*}{$\begin{array}{l}\mathrm{HC} \\
(N=24) \\
M \pm S D\end{array}$} & \multicolumn{4}{|c|}{ Statistical Analysis } \\
\hline & & & & $H$ & $p$ & $\eta p 2$ & $\begin{array}{l}\text { Interpretatio } \\
\mathrm{n} \text { of effect } \\
\text { sizes }\end{array}$ \\
\hline \multicolumn{8}{|l|}{ Kidsscreen-27: } \\
\hline $\begin{array}{l}\text { Physical } \\
\text { Wellbeing }\end{array}$ & $17.71 \pm 3.59$ & $18.5 \pm 3.61$ & $19.38 \pm 3.16$ & 3.740 & 0.155 & 0.04 & $\mathrm{~s}$ \\
\hline $\begin{array}{l}\text { Psychological } \\
\text { Wellbeing }\end{array}$ & $28.14 \pm 5.61$ & $31.86 \pm 2.28$ & $31.46 \pm 2.41$ & 3.258 & 0.197 & 0.16 & I \\
\hline $\begin{array}{l}\text { Parent \& } \\
\text { Autonomy }\end{array}$ & $31.14 \pm 3.53$ & $31.57 \pm 3.17$ & $31.479 \pm 2.95$ & 50.238 & 0.89 & 0 & $\mathrm{~s}$ \\
\hline $\begin{array}{l}\text { Peers \& Social } \\
\text { Support }\end{array}$ & $16.71 \pm 2.43$ & $17.43 \pm 2.38$ & $17.29 \pm 2.24$ & 0.705 & 0.719 & 0.01 & $\mathrm{~s}$ \\
\hline $\begin{array}{l}\text { School } \\
\text { Environment }\end{array}$ & $15.57 \pm 3.41$ & $17.07 \pm 1.86$ & $17.29 \pm 2.35$ & 1.033 & 0.599 & 0.06 & $\mathrm{~m}$ \\
\hline ChilD-S & $6.75 \pm 5.78$ & $4.07 \pm 3.29$ & $3.75 \pm 2.75$ & 1.895 & 0.395 & 0.09 & $\mathrm{~m}$ \\
\hline
\end{tabular}

Notes: $N=46$; degrees of freedom always $=2.41 ; p<.05$ statistically significant; effect sizes: small $(\mathrm{s})=.01>\eta p 2<.059$. medium $(\mathrm{m})=.06>\eta p<.139$. or large $(\mathrm{l})=\eta p \geq .14$

IBD-AD IBD in an active state of the disease, IBD-RE IBD in remission, $H C$ Healthy Control, ChilD-S Child Depression Screener

\section{Physical activity}

Table 4 shows that results on a subjective scale assessing the last 7 days, the IPAQ questionnaire did not indicate any statistically significant mean differences in self-reported PA between the three groups IBD AD, IBD-RE and HC. On a descriptive level, the IBD- AD group reported more vigorous physical activity (medium ES of .088) than the IBD-RE and HC groups. However, self-estimated fitness was lowest in the IBD-AD group (ES = .109). Mean grip strength did not differ statistically significantly between the three groups IBD-AD, IBD-RE and HC. 


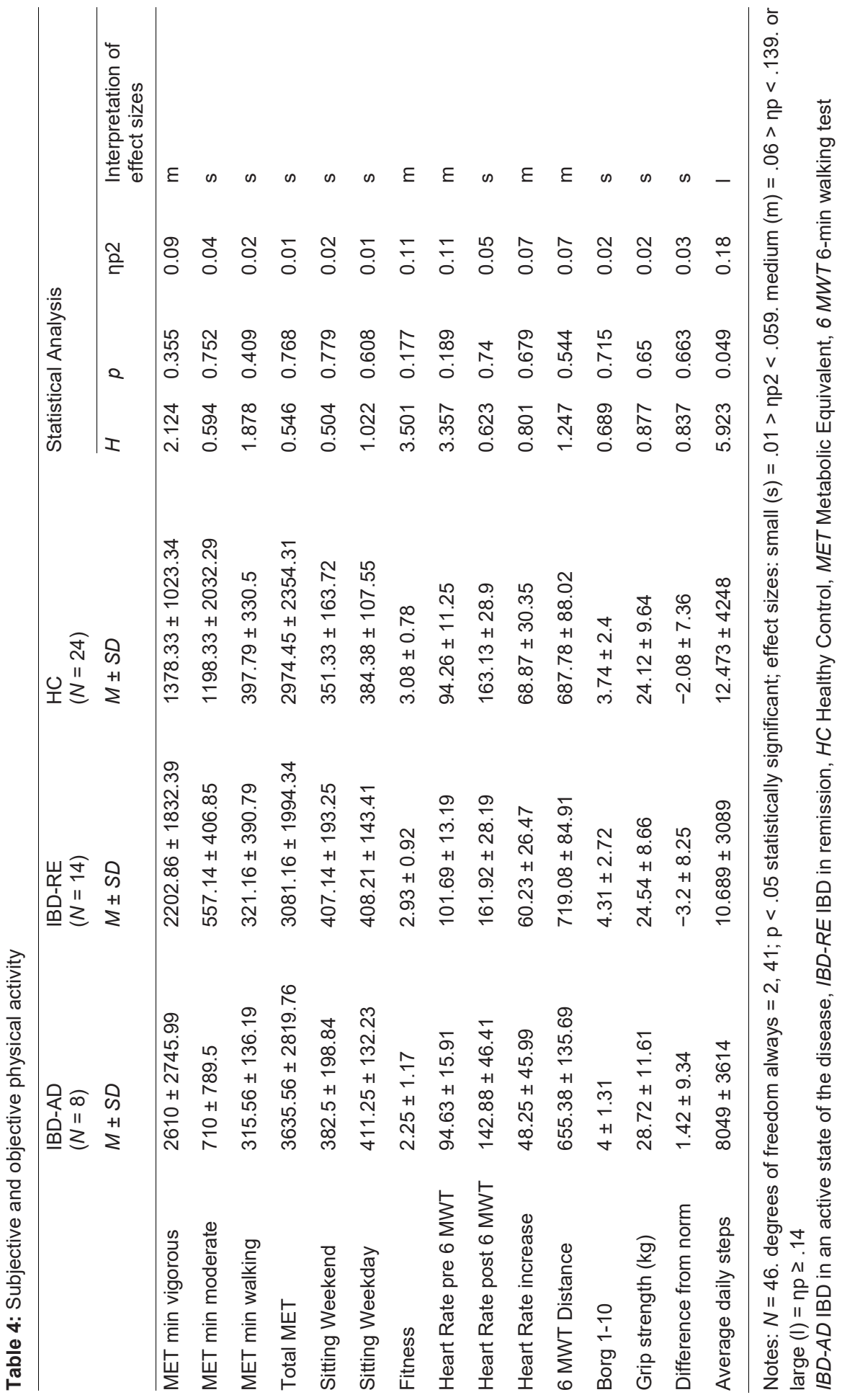


Even though statistically non-significant, the mean 6MWT results indicated descriptive differences, with IBD-AD patients achieving the shortest distance compared to IBD-RE and HC (medium ES = .070). The IBD-AD also had less increase in heart rate (medium ES $=.073$ ), but still rated the intensity of the test as high as the other two groups (small ES $=.018$ ). For the objective step counts as measured by the FitbitFlex®, a statistical significant mean difference $(p=.049)$ and a large effect size of .183 were observed; the average count was 8049 steps per day in the IBD-AD group, 10,689 steps per day in IBD-RE and 12,473 steps per day in the HC group. According to Mann-Whitney-U post-hoc tests, comparisons between groups were not significant.

\section{Discussion}

The key findings of the present study were that children and adolescents with IBD-AD had poorer psychological functioning than children and adolescents with IBD-RE and HC. Furthermore, they had a lower functional capacity (6MWT) and engaged less in objectively assessed physical activity (average steps per day) compared to children and adolescents with IBD in remission or age- and gendermatched healthy controls. The present pattern of results adds to the current literature in an important way, showing that medically well-adjusted children and adolescents with IBD do not differ from healthy controls with regard to psychological functioning and objective and subjective PA.

Three hypotheses were tested and each of these is considered now in turn.

Our first hypothesis was that there would be anthropometric differences; specifically, we expected that participants with IBD were smaller, lighter and to have greater inflammation, especially among the more severe cases of IBD. Our data partly supported this hypothesis. The IBD-AD group had a moderately lower BMI z-score, BMI percentile, z-score height for age and percentile height for age when compared to both other groups. Therefore, the present data are in accordance with previous findings [14, 30]. In general, $10-40 \%$ of children with IBD are affected by growth failure [14, 30] and impaired nutritional status [31]. Underlying reasons are anorexia, malabsorption, intestinal inflammation and corticosteroid usage [32]. Given that the data available do not allow a deeper understanding of the pattern of results, we speculate that current treatment strategies lead to good disease control and less growth retardation [33].

With the first hypothesis, we also expected to detect indicators for inflammation and disease-related influences in the blood values of IBD patients. This hypothesis was partially supported; scores for the inflammatory markers ESR, CRP and leukocytes were higher among those with IBD-AD. Two patients with active IBD and two in remission were anemic, as well as one participant in the control 


\section{Chapter 3}

group. In general, chronic anemia is prevalent and rapidly recurring in patients with IBD [34]. On the other hand, iron deficiency is a well-known phenomenon among many healthy female teenagers [35]. The small reported differences can be accounted to constant monitoring of blood values by the treating physician, who provides the patients with either oral or intravenous iron supplementation as soon as necessary. Looking at albumin values, it is not unusual to observe lower values in patients with IBD, as indicated by our findings. Patients with IBD might lose albumin due to increased gut permeability, even during symptom-free episodes [36]. However, we were unable to explain the low albumin values found in half of the healthy control participants. Common underlying reasons are undernourishment [37], decreased production due to liver disease [38] and increased excretion due to kidney problems [39], factors, which however could be ruled out by the supervising health professionals in the present study. Finally, the increased average hemoglobin among IBD-AD in comparison to $\mathrm{HC}$ was very surprising. The only possible explanation might be a blood clotting during blood sample taking since the hematocrit is elevated as well.

With the second hypothesis, we expected poorer psychological functioning in children and adolescents with IBD-AD compared to IBD-RE and healthy controls, and data did partially support this. Children and adolescents with increased disease activity had poorer psychological functioning in the area of psychological wellbeing. On the flipside, no other dimension of psychological functioning (physical functioning, autonomy and parent relations, social support and peers, and school experience) showed significant differences across the three groups, IBDAD, IBD-RE and HC. Therefore, the present data do not match the study by Herzer et al. [40], showing a lower overall HRQOL among patients with IBD. Further, the present data do not accord with those studies reporting impaired physical functioning [41], impaired family functioning [40], limited participation in social activities [7], lower emotional functioning [42], and problematic school experience and performance.

Previous evidence can be explained by the association between increased disease activity and lower psychological functioning. One may claim that greater disease severity might be accompanied by an increasing presence of disruptive gastrointestinal symptoms and abdominal pain, which in turn need to be treated with more robust and invasive methods. Such increasing disease burden might result in psychological distress [43]. Two further hypotheses are the inflammation-depression hypothesis and the brain gut hypothesis. These two hypotheses claim that the increased production of pro-inflammatory cytokines (TNF alpha) is known to affect the brain both, directly and indirectly, thereby increasing symptoms of depression [44, 45]. Further, there is a bi-directional relationship, since psychological stress in turn increases the likelihood of inflammation, which again increases the occurrence of depressive symptoms [42]. The pro-inflammatory 
effect of experimental stress has been confirmed in human studies, and at the same time, inflammatory markers have been found to be raised in depressed patients [46]. To a wider extent, the inflammatory, unpredictable and disruptive nature of severe IBD, if not properly treated, could lead to an increase in internalizing symptoms (e.g., anxiety and depression). In particular, given that the longterm course of the disease is characterized by progressive deterioration [47], young patients might be at increased risk for psychological distress.

While comparing children and adolescents in remission with the healthy control group, we did not find differences on any of the HRQOL dimensions. This can be attributed to the fact that children in remission are by definition symptom-free [11]. A recent publication by the SWISS IBD Cohort even reported higher psychological functioning in IBD patients than in controls. The authors attributed this unexpected finding to the excellent social support in the young patients' environment [41]. Walter et al. [48], on the basis of a careful examination of the literature, noted that older studies (1989 - 2004) reported higher depression rates; subsequent advances in treatment may be responsible for the lower levels of psychological distress observed more recently. Therefore, we suggest that screening and treatment for mental wellbeing should be implemented especially for those with increased disease activity and potentially weaker social support.

Our third hypothesis, following others [18, 49], was that physical activity levels (subjective and objective) would be lower in children and adolescents with IBD than in their healthy counterparts. While the three groups did not differ regarding subjectively assessed PA in our study, lower objectively assessed PA was indicated by the number of steps per day, especially in the IBD-AD group. Thus, the present data are in account with previous results, however based on objective measurements. Werkstetter et al. [14] also reported reduced amounts of steps per day in children and adolescents with IBD, while studies of adult patients have found that suffering from IBD tended to lead to a sedentary lifestyle [50]. An explanation might be that patients with active IBD are restricted and discouraged by unpredictable symptoms, physical restriction, inconvenience and discomfort [49]. Furthermore, we found that children and adolescence with active IBD perceived physical strain to be more vigorous as compared to IBD-RE and HC. This is reflected in their subjectively estimated greater intensity of the 6 MWT, while achieving less distance than the other groups and their extremely elevated selfreported vigorous PA levels over the last 7 days. This finding indicated a lower fitness in children and adolescents with active IBD, and was in line with findings of Ploeger et al. [13]. Children with IBD exhibited impaired aerobic and anaerobic exercise capacity, compared to reference values.

However, a lower amount of PA might be an issue for the following reasons. Generally, regular low intensity exercise is beneficial in reducing distress and improving quality of life in young patients with IBD [51]. A UK survey revealed that 
PA made patients with IBD feel better and healthier, boosted energy, reduced IBD symptoms, provided an alternative focus and fostered feelings of normality [49]. On a physiological level, recent findings indicated that IBD patients could experience anti-inflammatory effects from the myokines released during skeletal muscle contraction while exercising, thereby inhibiting the release of protective heat shock proteins (Hsps), which help to regulate inflammation and immunity [52]. It was further asserted that PA is an anabolic stimulus, reducing inflammation and positively affecting growth factors, i.e., IGF-I [53]. Additionally, Robbins et al. [49] argued that regular PA should be undertaken in IBD to help maintain bone mineral density and prevent osteoporosis. We should note, however, that exaggerated amounts of (high-intensity) PA might lead to an increase in symptoms. Vigorous PA such as distance running and endurance exercise commonly might cause gastrointestinal discomfort including nausea, heartburn, and even gastrointestinal bleeding in patients with IBD [54]. By contrast, moderate PA seems to be useful in improving many aspects of the lives of patients with IBD. Nonetheless, a fear of symptoms exacerbation may be an indication that patients lack the impetus to start PA, and clinicians may be reluctant to prescribe PA, even though it might serve as a protective and preventive factor with respect to IBD. Collectively, we claim that regular moderate PA should receive greater attention in scientific intervention studies as adjuvant to prescribed pharmacotherapies.

Despite the novelty of the findings and the application of internationally validated and accepted questionnaires and objective measures, several limitations warrant against an overgeneralization of the present findings. First, the relatively small sample, especially the small number of children in an active disease state, created difficulties in detecting statistically significant effects. Though we also relied on effect sizes, which are not sensitive to sample size. Second, the recruitment of the sample was restricted to the German speaking part of Switzerland. Of these patients, only participants willing and able to participate were assessed. Even though we tried to cover an extensive range of factors, it was not possible to control for all confounders, such as microbial or environmental conditions, which might have influenced two or more outcomes in the same or in opposite directions. In this context, one suggestion for future studies might be the assessment of objective disease severity, fecal calprotectin as another objective measure. Otherwise we would suggest extending the cross sectional design to include lifestyle intervention studies and their supportive effect in the disease coping process. Last, we did not distinguish between patients with CD and UC; whereas for diagnostic reasons such an approach would have been easy to follow and justify, we decided to split patients into IBD-AD and IBD-RE, as suggested by recent research such as Reigada et al. [42, 55]. 


\section{Conclusion}

The pattern of results suggests that effective medical treatment of IBD in children and adolescents is associated with favorable physiological parameters, psychological dimensions and PA. Psychological counselling of children and adolescents with severe IBD seem to be advised in addition to standard treatment schedules.

\section{Abbreviations}

6 MWT: Six minute walking test; CD: Crohn's disease; CDAl: Crohn's disease activity index; ChilD-S: Child Depression Screener; CRP: C-reactive protein; ERS: Erythrocyte sedimentation rate; ES: Effect size; HC: Healthy control;HR: Heart rate; HRQOL: Health related quality of life; Hsps: Heat shock proteins; IBD: Inflammatory bowel diseases; IBD-AD: IBD in an active state of the disease; IBDRE: IBD in remission; MET: Metabolic equivalent; PA: Physical activity; PIBD: Pediatric IBD; PUCA: Pediatric ulcerative colitis activity index; UC: Undefined colitis

\section{Acknowledgements}

We thank the Kantonsspital Aarau for providing additional recruitment options. We are also grateful to Noe Stoll for support during data collection and data entry. Finally, we thank Nick Emler (Surrey, UK) for proofreading the manuscript and Harald Seelig for supporting the statistical analysis.

\section{Funding}

We thank the Freiwillige Akademische Gesellschaft Basel (FAG, Basel, Switzerland) for financially supporting the project.

\section{Availability of data and materials}

The datasets used and/or analysed during the current study available from the corresponding author on reasonable request. 


\section{Chapter 3}

\section{Authors' contributions}

Conception and Design, LM, MG, RIF, CL, NK, EHT, SB; Acquisition of data, LM, M.G, RIF, CL, NK; Analysis and interpretation of data: LM, MG, RF, CL, NK, EHT, $\mathrm{SB}$; Drafting of manuscript: LM, MG, RIF, CL, NK, EHT, SB. All authors read and approved the final manuscript.

\section{Ethics approval and consent to participate}

The present study was approved by the local ethical committee (Ethikkommission Nordwest- und Zentralschweiz (EKNZ): 2014:220), and was also conducted in accordance to the ethical principles laid down in Declaration of Helsinki (Trial registration number: NCT02264275). Voluntary participation was encouraged and informed consent to participate in the study was obtained from each patient. All participants and their legal guardian had to consent the study participation in written format. Patient records or information was anonymized and deidentified prior to analysis.

\section{Consent for publication}

Not applicable.

\section{Competing interests}

The authors declare that they have no competing interests. 


\section{References}

1 Levine $\mathrm{A}$, de $\mathrm{Bie} \mathrm{Cl}$, Turner $\mathrm{D}$, et al. Atypical disease phenotypes in pediatric ulcerative colitis: 5-year analyses of the EUROKIDS registry. Inflamm Bowel Dis. 2013;19:370-7.

2 Lemberg DA, Day AS. Crohn disease and ulcerative colitis in children: an update for 2014. J Paediatr Child Health. 2015;51:266-70.

3 Loddo I, Romano C. Inflammatory bowel disease: genetics, epigenetics, and pathogenesis. Front Immunol. 2015;6 Available from: http://journal.frontiersin.org/Article/10.3389/fimmu.2015.00551/ abstract. Cited 21 Mar 2016

4 Braegger CP, Ballabeni P, Rogler D, Vavricka SR, Friedt M, Pittet V, et al. Epidemiology of inflammatory bowel disease: is there a shift towards onset at a younger age? J Pediatr Gastroenterol Nutr. 2011;53:141-4.

5 Pappa H, Thayu M, Sylvester F, Leonard M, Zemel B, Gordon C. Skeletal health of children and adolescents with inflammatory bowel disease. J Pediatr Gastroenterol Nutr. 2011;53:11-25.

6 Rosen MJ, Dhawan A, Saeed SA. Inflammatory bowel disease in children and adolescents. JAMA Pediatr. 2015;169:1053.

7 Engstrom I. Mental-health and psychological functioning in children and adolescents with inflammatory bowel-disease: a comparison with children having other chronic illnesses and with healthy-children. J Child Psychol Psychiatry. 1992;3:563-82.

8 Ross SC, Strachan J, Russell RK, Wilson SL. Psychosocial functioning and health related quality of life in paediatric inflammatory bowel disease: a systematic review. J Pediatr Gastroenterol Nutr. 2011;53:480.

9 Vaisto T, Aronen ET, Simola P, Ashorn M, Kolho KL. Psychosocial symptoms and competence among adolescents with inflammatory bowel disease and their peers. Inflamm Bowel Dis. 2010;1:27-35.

10 Rabizadeh S, Dubinsky M. Update in pediatric inflammatory bowel disease. Rheum Dis Clin N Am. 2013;39:789-99.

11 Mikocka-Walus A, Knowles SR, Keefer L, Graff L. Controversies revisited: a systematic review of the comorbidity of depression and anxiety with inflammatory bowel diseases. Inflamm Bowel Dis. 2016;22:752-62.

12 Spekhorst LM, Hummel TZ, Benninga MA, van Rheenen PF, Kindermann A. Adherence to oral maintenance treatment in adolescents with inflammatory bowel disease. J Pediatr Gastroenterol Nutr. 2016;62:264-70.

13 Ploeger $\mathrm{H}$, Obeid J, Nguyen $\mathrm{T}$, Takken $\mathrm{T}$, Issenman $\mathrm{R}$, de Greef $\mathrm{M}$, et al. Exercise and inflammation in pediatric Crohn's disease. Int J Sports Med. 2012;33:671-9.

14 Werkstetter KJ, Ullrich J, Schatz SB, Prell C, Koletzko B, Koletzko S. Lean body mass, physical activity and quality of life in paediatric patients with inflammatory bowel disease and in healthy controls. J Crohns Colitis. 2012;6:665-73.

15 Loprinzi PD, Cardinal BJ. Association between objectively-measured physical activity and sleep, NHANES 2005-2006. Ment. Health and Phys Act. 2011;4:65-9.

16 Gerber M, Lindwall M, Lindegård A, Börjesson M, Jonsdottir IH. Cardiorespiratory fitness protects against stress-related symptoms of burnout and depression. Patient Educ Couns. 2013;93:14652.

17 Hummel TZ, Tak E, Maurice-Stam H, Benninga MA, Kindermann A, Grootenhuis MA. Psychosocial developmental trajectory of adolescents with inflammatory bowel disease. J Pediatr Gastroenterol Nutr. 2013;57:219-24.

18 Chan D, Robbins H, Rogers S, Clark S, Poullis A. Inflammatory bowel disease and exercise: results of a Crohn's and Colitis UK survey. Frontline Gastroenterol. 2014;5:44-8.

19 Taylor RW, Jones IE, Williams SM, Goulding A. Evaluation of waist circumference, waist-to-hip ratio, and the conicity index as screening tools for high trunk fat mass, as measured by dualenergy X-ray absorptiometry, in children aged 3-19 y. Am J Clin Nutr. 2000;72:490-5. 


\section{Chapter 3}

20 WHO Multicentre Growth Reference Study Group. WHO Child growth standards: length/heightfor-age, weight-for-age, weight-for-length, weight- for-height and body mass index-for-age: methods and development. Geneva: World Health Organization; 2006.

21 Ravens-Sieberer U, Herdman M, Devine J, Otto C, Bullinger M, Rose M, et al. The European KIDSCREEN approach to measure quality of life and well- being in children: development, current application, and future advances. Qual Life Res. 2014;23:791-803.

22 Frühe B, Allgaier A-K, Pietsch K, Baethmann M, Peters J, Kellnar S, et al. Children's Depression Screener (ChilD-S): development and validation of a depression screening instrument for children in pediatric care. Child Psychiatry Hum Dev. 2012;43:137-51.

23 IPAQ. Guidelines for data processing and analysis of the International Physical Activity Questionnaire (IPAQ) - short and long forms, revised on November 2005. 2005. Available from: http://www.ipaq.ki.se/ scoring.pdf. Cited 15 Mar 2010.

24 Hagströmer M, Bergman P, De Bourdeaudhuij I, Ortega FB, Ruiz JR, Manios Y, et al. Concurrent validity of a modified version of the International Physical Activity Questionnaire (IPAQ-A) in European adolescents: the HELENA study. Int J Obes. 2008;32:S42-8.

25 Mathiowetz V, Wiemer DM, Federman SM. Grip and pinch strength: norms for 6-to 19-year-olds. Am J Occup Ther. 1986;40:705-11.

26 Solway S, Brooks D, Lacasse Y, Thomas S. A qualitative systematic overview of the measurement properties of functional walk tests used in the cardiorespiratory domain. Chest. 2001;119:256-70.

27 Hassan J, van der Net J, Helders PJM, Prakken BJ, Takken T. Six-minute walk test in children with chronic conditions. Br J Sports Med. 2010;44:270-4.

28 Meltzer LJ, Hiruma LS, Avis K, Montgomery-Downs H, Valentin J. Comparison of a commercial accelerometer with polysomnography and actigraphy in children and adolescents. Sleep. 2015;38:1323-30.

29 Cohen J. Statistical power analysis for the behavioral sciences. 2nd ed. Hillsdale: N.J: Routledge; 1988.

30 Dubinsky M. Special issues in pediatric inflammatory bowel disease. World J Gastroenterol. 2008; $14: 413$.

31 Gasparetto M. Crohn's disease and growth deficiency in children and adolescents. World J Gastroenterol. 2014;20:13219.

32 Shamir R. Nutritional aspects in inflammatory bowel disease. J Pediatr Gastroenterol Nutr. 2009;48:S86-8.

33 De Greef E, Hoffman I, Smets F, Van Biervliet S, Bontems P, Hauser B, et al. Paediatric Crohn's disease: disease activity and growth in the BELCRO cohort after 3 years follow-up. J Pediatr Gastroenterol Nutr. 2016;63:253.

34 Kulnigg S, Teischinger L, Dejaco C, Waldhör T, Gasche C. Rapid recurrence of IBD-associated anemia and iron deficiency after intravenous iron sucrose and erythropoietin treatment. Am J Gastroenterol. 2009;12:1460-7.

35 Looker AC, Dallman PR, Carroll MD, Gunter EW, Johnson CL. Prevalence of iron deficiency in the United States. JAMA. 1997;12:973-6.

36 Michielan A, D'Incà R. Intestinal permeability in inflammatory bowel disease: pathogenesis, clinical evaluation, and therapy of leaky gut. Mediat Inflamm. 2015;2015:628157.

37 Vermeire S, Van Assche G, Rutgeerts P. Laboratory markers in IBD: useful, magic, or unnecessary toys? Gut. 2006;55:426-31.

38 Bernardi M, Maggioli C, Zaccherini G. Human albumin in the management of complications of liver cirrhosis. Crit Care. 2012;16:211.

39 Birn $\mathrm{H}$, Christensen El. Renal albumin absorption in physiology and pathology. Kidney Int. 2006;69:440-9.

40 Herzer M, Denson LA, Baldassano RN, Hommel KA. Patient and parent psychosocial factors associated with health-related quality of life in pediatric inflammatory bowel disease. J Pediatr Gastroenterol Nutr. 2011;52:295-9. 
41 Rogler D, Fournier N, Pittet V, Bühr P, Heyland K, Friedt M, et al. Coping is excellent in Swiss children with inflammatory bowel disease: results from the Swiss IBD cohort study. J Crohns Colitis. 2014;8:409-20.

42 Goodhand JR, Wahed M, Mawdsley JE, Farmer AD, Aziz Q, Rampton DS. Mood disorders in inflammatory bowel disease: relation to diagnosis, disease activity, perceived stress, and other factors. Inflamm Bowel Dis. 2012;18:2301-9.

43 Gray WN, Denson LA, Baldassano RN, Hommel KA. Disease activity, behavioral dysfunction, and health-related quality of life in adolescents with inflammatory bowel disease. Inflamm Bowel Dis. 2011;17:1581-6.

44 Raison CL, Capuron L, Miller AH. Cytokines sing the blues: inflammation and the pathogenesis of depression. Trends Immunol. 2006;27:24-31.

45 van den Brink G, Stapersma L, El Marroun H, Henrichs J, Szigethy EM, Utens EM, et al. Effectiveness of disease-specific cognitive-behavioural therapy on depression, anxiety, quality of life and the clinical course of disease in adolescents with inflammatory bowel disease: study protocol of a multicentre randomised controlled trial (HAPPY-IBD). BMJ Open Gastroenterol. 2016;3:e000071.

46 Miller $\mathrm{AH}$, Raison $\mathrm{CL}$. The role of inflammation in depression: from evolutionary imperative to modern treatment target. Nat Rev Immunol. 2016;16:22-34.

47 Pittet V, Juillerat P, Mottet C, Felley C, Ballabeni P, Burnand B, et al. Cohort profile: the Swiss Inflammatory Bowel Disease Cohort Study (SIBDCS). Int J Epidemiol. 2009;38:922-31.

48 Walter JG, Kahn SA, Noe JD, Schurman JV, Miller SA, Greenley RN. Feeling fine: anxiety and depressive symptoms in youth with established IBD. Inflamm Bowel Dis. 2016;22:402-8.

49 Robbins $\mathrm{H}$, Poullis A, Rogers S. Inflammatory bowel disease and exercise- preliminary results of a Crohn's and colitis UK survey. Gastroenterol Today. 2012;22:62-3.

50 Narula N, Fedorak RN. Exercise and inflammatory bowel disease. Can J Gastroenterol. 2008;22:497-504.

$51 \mathrm{Ng} \mathrm{V}$, Millard W, Lebrun C, Howard J. Low-intensity exercise improves quality of life in patients with Crohn's disease. Clin J Sport Med. 2007;17:384-8.

52 Chen Y, Noble EG. Is exercise beneficial to the inflammatory bowel diseases? An implication of heat shock proteins. Med Hypotheses. 2009;72:84-6.

53 Sanderson IR. Growth problems in children with IBD. Nat Rev Gastroenterol Hepatol. 2014;11:601.

54 Colditz GA, Cannuscio CC, Frazier AL. Physical activity and reduced risk of colon cancer: implications for prevention. Cancer Causes Control. 1997;8:649-67.

55 Reigada LC, Hoogendoorn CJ, Walsh LC, Lai J, Szigethy E, Cohen BH, et al. Anxiety symptoms and disease severity in children and adolescents with Crohn disease. J Pediatr Gastroenterol Nutr. 2015;60:30-5. 



\section{Chapter 4}

\section{Impaired objective and subjective sleep in children and adolescents with inflammatory bowel disease compared to healthy controls}

Published as: Mählmann L, Gerber M, Furlano RI, Legeret C, Kalak N, Holsboer-Trachsler E, Brand S. Impaired objective and subjective sleep in children and adolescents with inflammatory bowel disease compared to healthy controls. Sleep Med. 2017 Nov;39:25-31. doi: 10.1016/j.sleep.2017.08.015. 


\section{Chapter 4}

\section{Abstract}

Objective: Poor sleep and higher inflammation markers are associated, and impaired sleep quality is common among patients with inflammatory bowel disease (IBD). However, information on sleep among children and adolescents with IBD is currently lacking. The aims of the present study were to compare subjective and objective sleep of children and adolescents with IBD with healthy controls and to shed more light on the relationship between sleep and inflammation. We expected that poor sleep, as assessed via sleep electroencephalography recordings, would be observed among participants with IBD, but particularly among participants in an active state of disease. Furthermore, we expected that poor sleep and higher inflammatory markers would be associated.

Methods: A total of 46 children and adolescents participated in the study; 22 were diagnosed with IBD (mean age: 13.88 years, 44\% female). The IBD group was divided into a medically well adjusted "remission-group" (IBD-RE; $n=14$ ) and a group with an "active state of disease" (IBD-AD; $n=8$ ). Healthy controls $(\mathrm{HC} ; \mathrm{n}=24)$ were age and gender matched. Participants completed self-rating questionnaires for subjective sleep disturbances. Anthropometric data, acute and chronic inflammatory markers (C-reactive protein [CRP] and erythrocyte sedimentation rate $[E S R]$ ) and objective sleep were considered.

Results: Compared to HC and IBD-RE, IBD-AD patients showed impaired objective sleep patterns (e.g., more awakenings, longer sleep latency, and reduced stage 3 sleep). Linear relationships described the correlation between higher ESR and more stage 4 (minutes, percentage) sleep. Nonlinear relationships described the relation between ESR and subjective sleep quality (inverse Ushaped) and between CRP and sleep latency (U-shaped).

Conclusion: In children and adolescents with an active IBD, objective sleep was impaired and overall sleep quality and inflammation indices were associated in a complex manner. It seems advisable to include assessment of subjective sleep quality in the care of pediatric IBD patients as an additional indicator for objective sleep disturbances and inflammation. 


\section{Introduction}

The term "inflammatory bowel disease" (IBD) refers to a chronic, noncommunicable disease taking one of three major forms of spontaneous intestinal inflammation. In addition to Crohn's disease (CD) and ulcerative colitis (UC), 10-15\% of patients with inflammatory bowel disease experience a colitis that must initially be designated as indeterminate because its macroscopic and microscopic features do not allow a clear categorization as either UC or colonic CD [1].

The underlying causes of IBD have not been fully identified; however, current theories acknowledge interactions between the intestinal gut microflora and the mucosa, especially in predisposed hosts, as responsible underlying mechanisms. Genetic factors seem to contribute only partially, while there is growing recognition that the microbial and environmental dimensions play an important role [2,4].

IBD manifests during childhood or adolescence in at least $20 \%$ of patients, often alternating between active and quiescent states. The burden of illness imposed on children and adolescents affected by these chronic relapsing disorders may be considerable [5]. Once a diagnosis is confirmed, the goals of anti-inflammatory therapy are to eliminate symptoms, restore growth and bone health, normalize quality of life and psychosocial functioning, prevent complications, and minimize the adverse effects of medications [6]. However, currently available antiinflammatory pharmaceuticals do not achieve complete effectiveness and can have severe side effects. Thus, while some children and adolescents with IBD are in a symptom-free remission, others are still in an active state of the disease. In pediatric patients, severity of the disease is assessed regularly on the basis of Paediatric Ulcerative Colitis Activity Index (PUCAI) scores and by the Paediatric Crohn's Disease Activity Index (PCDAI) (clinical scores). Using these parameters, participants in the present study were divided between those with medically optimized treatment (IBD in remission), the IBD-RE group, and those without optimized medical treatment and therefore still in an active disease state, the IBDAD group.

As regards the association between poor sleep and inflammation markers, in the nonclinical population, Fernandez-Mendoza et al. [7] recently showed that, in a cohort of 378 adolescents, both symptoms of insomnia and objective short sleep were associated with higher C-reactive protein (CRP) and other inflammatory markers. This pattern of results is consistent with a recent meta-analysis [8] of 72 studies comprising about 50000 middle-aged adults. This meta-analysis indicated poor sleep to be associated with increased levels of CRP.

In addition, it has been argued that in acute states of inflammation, slow-wave sleep (SWS) might serve a restorative function by activating the immune system. In a meta-analysis by Ranjbaran et al. [9], this was illustrated comparing sleep parameters under inflammatory conditions. For asthma, rheumatoid arthritis, and 
systemic lupus erythematosus, increased sleep latency, frequent awakening, and daytime sleepiness were reported on a subjective scale, and increased SWS and sleep latency were detected for all three conditions by assessments using polysomnography.

Regarding the association between sleep and IBD, the following studies have thus far been carried out. Among adults with IBD, Ranjbaran et al. [9] used the Pittsburgh Sleep Quality Index (PSQI) to assess the subjective sleep quality of 199 patients with IBD and of healthy controls. Among those with IBD, significantly prolonged sleep latency, frequent sleep fragmentation, greater use of sleeping pills, decreased daytime energy, increased tiredness, and poor overall sleep quality were observed. Furthermore, an association was found between severity of symptoms and sleep quality during the previous night. Furthermore, adult IBD patients in clinical remission suffering from sleep disorders were more likely to experience flare-ups and subclinical disease activity compared to patients without sleep disturbances [10-13]. Finally, a single study using objective sleep assessment has been conducted with an adult sample; by investigating subjective sleep complaints and using a single overnight polysomnography, Keefer et al. [14] found greater subjective sleep impairment in 16 patients with IBD (seven males, mean age: $41.44 \pm 13.31$ years) than in seven healthy controls, while sleep electroencephalography (EEG) recordings showed shorter sleep duration and lower sleep efficiency in the IBD patients than in the healthy controls.

With regard to research on sleep among children and adolescents with IBD, in a cross-sectional study Werkstetter et al. [15] assessed self-reported sleep duration. They found greater overall sleep duration in 39 children and adolescents with IBD (24 males, $15.1 \pm 2.9$ years) than in 39 healthy controls $(15.2 \pm 3.1$ years). Unfortunately, the study focused only on sleep duration, which may not accurately reflect the degree to which sleep is restoring. Pirinen et al. [16] conducted a more detailed sleep assessment in a cross-sectional survey of the parents of 160 Finnish children with IBD (85 males, $15.4 \pm 2.2$ years); these parents commented on their children's sleep and reported that their children experienced greater sleep problems, more nightmares, increased sleep duration, and overtiredness when compared to the reports from parents of 236 healthy control subjects. Of the children with IBD, however, only those with a severe disease course confirmed their parents' reports; participants also described how disease-related symptoms affected their quality of sleep. In their second study, Pirinen et al. [11] noted that the self-reported poor sleep of children with IBD was associated with various psychosocial symptoms, including anxious and depressive mood, aggressive behavior, and attention problems.

Overall, we note that research on sleep among children and adolescents with IBD has so far relied on subjective sleep measurements; evidence based on objective assessment of sleep is missing. We further observe that previous studies 
did not distinguish between medically well-adjusted pediatric patients ("in remission") and those not yet medically well adjusted and thus currently in an active disease state.

The present study aimed at filling some gaps in the literature. More specifically, the main goal of the present investigation was to explore the differences in both subjective and objective sleep between children and adolescents in an active state of disease (IBD-AD), those in remission (IBD-RE), and healthy controls (HC), and to examine the associations of both subjective and objective sleep parameters with inflammatory indices (CRP, ESR).

The following four hypotheses were formulated. First, on the basis of previous studies $[10,13,17,18]$, we expected lower subjective sleep quality among children and adolescents in an active state of the disease (IBD-AD) than either children in remission (IBD-RE) or healthy controls. Second, following Keefer et al. [14], Fernandez- Mendoza et al. [7], and Irwin et al. [8], we expected poorer objective sleep patterns, as assessed by EEG recordings, in children and adolescents in the IBD-AD, as compared to the IBD-RE and HC-group. Third, following Fernandez-Mendoza et al. [7], and Irwin et al. [8], among children with IBD, we expected a relationship between poor subjective sleep and higher inflammatory markers (CRP and ESR). Fourth, and again following Fernandez-Mendoza et al. [7], we expected poor objective sleep patterns (such as shorter sleep durations, longer sleep onset latency, and more awakenings after sleep onset) to be associated with higher inflammatory markers (CRP and ESR) among children with IBD.

\section{Methods}

\section{Procedure}

A total of 47 children and adolescents participated in the study; 23 were diagnosed with IBD and 24 were age- and gender-matched healthy controls. All participants were invited to the hospital for assessments of height, weight, and waist circumference. Participants' blood values were collected, and PCDAI and PUCAI were assessed; participants also filled out a questionnaire assessing subjective sleep. Objective sleep quality was assessed at home, the night before a regular school day, using EEG. All data were collected by two trained research assistants and supervised by a medical doctor.

The present study was approved by the local ethics committee (EKNZ: 2014:220), and was conducted in accordance with the ethical principles laid down in the Declaration of Helsinki (trial registration number: NCT02264275). 


\section{Chapter 4}

\section{Sample}

A total of 46 children and adolescents agreed to participate in the study. Of these, 22 were diagnosed with IBD (mean age: 13.88 years, standard deviation [SD] $3.11,44 \%$ female) at the University Children's Hospital, Basel (UKBB, Basel, Switzerland). On the basis of regularly collected PUCAI scores and PCDAI (clinical) scores, the IBD group was divided into a medically well-adjusted "remissiongroup" ( $n=14$; IBD-RE) and a group with an "active state of disease" ( $n=8$; IBDAD) [19]. Healthy controls $(n=24 ; H C)$ were age and gender matched (mean age: 12.38 years, SD $3.24,62.5 \%$ female). All participants were informed about the study aims and the voluntary and confidential basis of their participation. Informed consent forms were signed both by participants and by their legal guardians.

Characteristics of the participants, subdivided into the three groups of IBDAD, IBD-RE and HC, are represented in Table 1.

Table 1: Socio-demographic, illness-related and anthropometric data, separately for children and adolescents in an active state of disease (IBD-AD), in remission (IBD-RE), and for healthy controls (HC) $(N=44)$.

\begin{tabular}{|c|c|c|c|}
\hline & $\begin{array}{l}\text { IBD-AD } \\
(n=7) \\
(\text { Mean } \pm \text { SD) }\end{array}$ & $\begin{array}{l}\text { IBD-RE } \\
(n=14) \\
(\text { Mean } \pm \text { SD) }\end{array}$ & $\begin{array}{l}\mathrm{HC} \\
(n=23) \\
(\text { Mean } \pm \mathrm{SD})\end{array}$ \\
\hline Age (y) & $14.69 \pm 3.25$ & $13.23 \pm 2.96$ & $12.38 \pm 3.24$ \\
\hline Female & $4(50 \%)$ & $6(42.9 \%)$ & $15(62.5 \%)$ \\
\hline \multicolumn{4}{|l|}{ IBD } \\
\hline Ulcerative colitis & $3(37.5 \%)$ & $4(28.6 \%)$ & \\
\hline Crohn's disease & $5(62.5 \%)$ & $7(50 \%)$ & \\
\hline Undefined colitis & & $3(21.4 \%)$ & \\
\hline Time since diagnosis & $111.54 \pm 83.35$ & $124.74 \pm 88.19$ & \\
\hline Waist circumference $(\mathrm{cm})$ & $67.63 \pm 9.04$ & $65.65 \pm 7.17$ & $64.48 \pm 8.29$ \\
\hline Difference from norm & $-9.81 \pm 6.15$ & $-8.68 \pm 4.95$ & $-8.82 \pm 6.00$ \\
\hline Height $(\mathrm{cm})$ & $153.48 \pm 17.53$ & $152.69 \pm 12.02$ & $152.26 \pm 18.00$ \\
\hline Weight to height (\%) & $44.08 \pm 3.07$ & $42.94 \pm 2.13$ & $42.77 \pm 3.49$ \\
\hline Weight $(\mathrm{kg})$ & $45.28 \pm 15.85$ & $42.79 \pm 12.83$ & $44.37 \pm 15.75$ \\
\hline BMI & $18.6 \pm 3.37$ & $17.91 \pm 2.78$ & $18.36 \pm 3.16$ \\
\hline BMI z-score & $-0.59 \pm 0.79$ & $-0.34 \pm 0.95$ & $-0.19 \pm 0.87$ \\
\hline Percentile BMI vs age & $31.98 \pm 24.31$ & $39.82 \pm 26.23$ & $43.89 \pm 26.43$ \\
\hline z-Score height vs age & $-0.91 \pm 1.73$ & $0.02 \pm 0.86$ & $0.19 \pm 1.01$ \\
\hline Percentile height vs age & $32.04 \pm 32.99$ & $52.91 \pm 25.71$ & $54.83 \pm 31.53$ \\
\hline
\end{tabular}

$\mathrm{BMI}$, body mass index; $\mathrm{HC}$, healthy control; IBD, inflammatory bowel disease; IBD- AD, IBD in an active state of the disease; IBD-RE, IBD in remission; SD, standard deviation. 


\section{Measures}

\section{Subjective sleep assessment}

Participants completed the Insomnia Severity Index (ISI) [20], a screening tool for insomnia. The seven items, answered on five- point rating scales $(0=$ not at all, $4=$ very much), refer to difficulty in falling asleep, difficulty maintaining sleep, increased day-time sleepiness, and worrying about sleep. The validity of this instrument has previously been established for German-speaking children and adolescents [21]. The higher the overall score, the more the respondent is assumed to suffer from sleep disturbances (Cronbach's $\alpha=0.88$ ).

\section{Objective sleep assessment}

Sleep EEG recordings were made at home using a three-channel EEG device (Fp2-A1, C3-A2, and C4-A1; electrooculogram; electromyogram; Somnowatch; Randersacker, Germany) [17]. On the day of the recording night, participants were attending regular school schedules. Participants were also requested to adhere to their normal evening routines. Two experienced raters visually analyzed sleep polygraphs according to the standard procedures [22]. The device was applied at around 20:00 by a trained researcher. The device provides assessment of total sleep time, sleep efficiency, sleep onset latency, stages 1-4 (minutes and percentage), rapid eye movement (REM) sleep, and number and times of awakenings after sleep onset.

\section{Blood values and inflammatory markers}

Using the finger-prick technique, biochemical parameters were assessed for two inflammatory indices, CRP $(\mathrm{g} / \mathrm{dL})$ and ESR $(\mathrm{mm} / \mathrm{h})$.

\section{Statistical analyses}

The IBD-AD, IBD-RE, and HC groups were compared using one- way univariate analyses of variance (ANOVAs) with subjective and objective sleep patterns, blood values, and inflammatory markers as dependent variables. Due to the heterogeneity of age distribution, age was introduced as a covariate in the analyses. Post hoc tests after Bonferronie Holm corrections for $p$ values were performed to examine differences among the three specific groups. Substantive significance with effect sizes for ANOVAs were reported as partial eta-squared $\left[\eta^{2}\right]$ and interpreted as follows: mall $(\mathrm{S})=.01>\eta^{2}<.059$, medium $(\mathrm{M})=.06>\eta^{2}<.139$, or large $(L)=\eta^{2} \geq$.14. [23]. Pearson correlations and nonlinear correlations were used to examine associations between objective sleep parameters and the inflammation parameters CRP and ESR. Interpretation of correlation coefficients 


\section{Chapter 4}

followed Cohen [23]; small: $|0.1|-|0.3|$; moderate: $|0.3|-|0.5|$; large: $|0.5|$ $|1.0|$. Across all analyses, the nominal level of statistical significance was set at alpha $p<0.05$. All statistics were performed with SPSS 22.0 for Windows (IBM Corporation, Armonk, NY, USA).

\section{Results}

All descriptive and inferential statistical indices are reported in the tables. All tables compare the three groups, IBD-AD, IBD-RE, and HC. Table 1 provides an overview of the socio-demographic, illness- related, and anthropometric sample characteristics.

\section{Subjective sleep}

Table 2 indicates that ISI scores did not significantly differ across the three groups. All effect sizes were small.

\section{Objective sleep}

As shown in Table 2, compared to the IBD-RE and HC groups, children and adolescents with IBD-AD were awake for significantly longer after sleep onset. They also had significantly less time in stage 3 and a smaller percentage of stage 3 sleep compared to IBD-RE and HC. Descriptively, increased sleep onset latency in IBD-AD, compared to IBD-RE and HC, corresponded to a medium effect BMI, body mass index; HC, healthy control; IBD, inflammatory bowel disease; IBD-AD, IBD in an active state of the disease; IBD-RE, IBD in remission; SD, standard deviation, size, and IBD-RE spent less time in stage 4 (\%) than either of the other groups (medium effect size).

\section{Inflammatory markers}

Table 3 indicates that there were no group differences for chronic inflammation (ESR). Concerning CRP, descriptively higher values were found in the IBD-AD and IBD-RE groups than among the healthy controls. Despite a medium-sized effect, the difference did not reach significance $(p=0.08)$. 


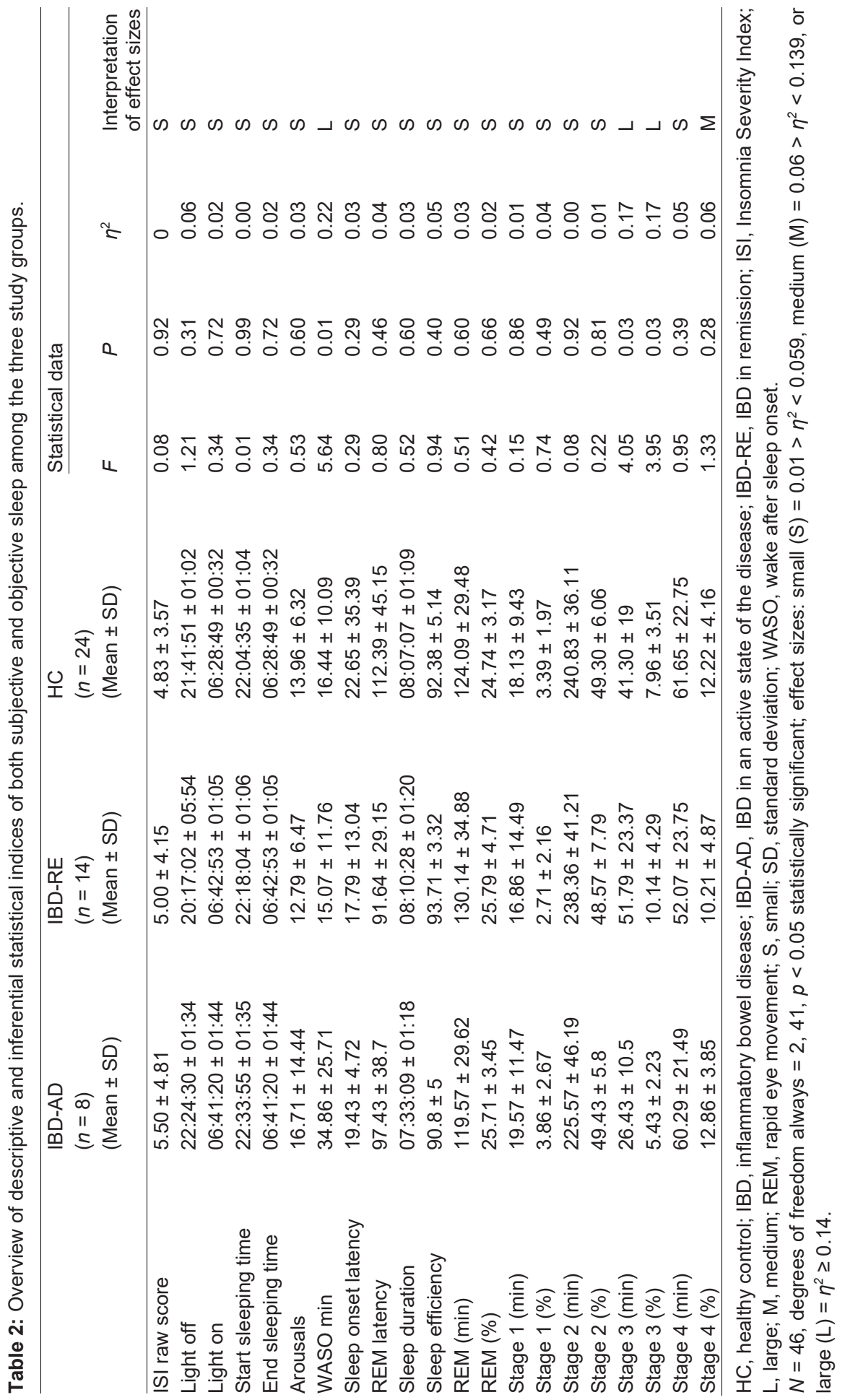




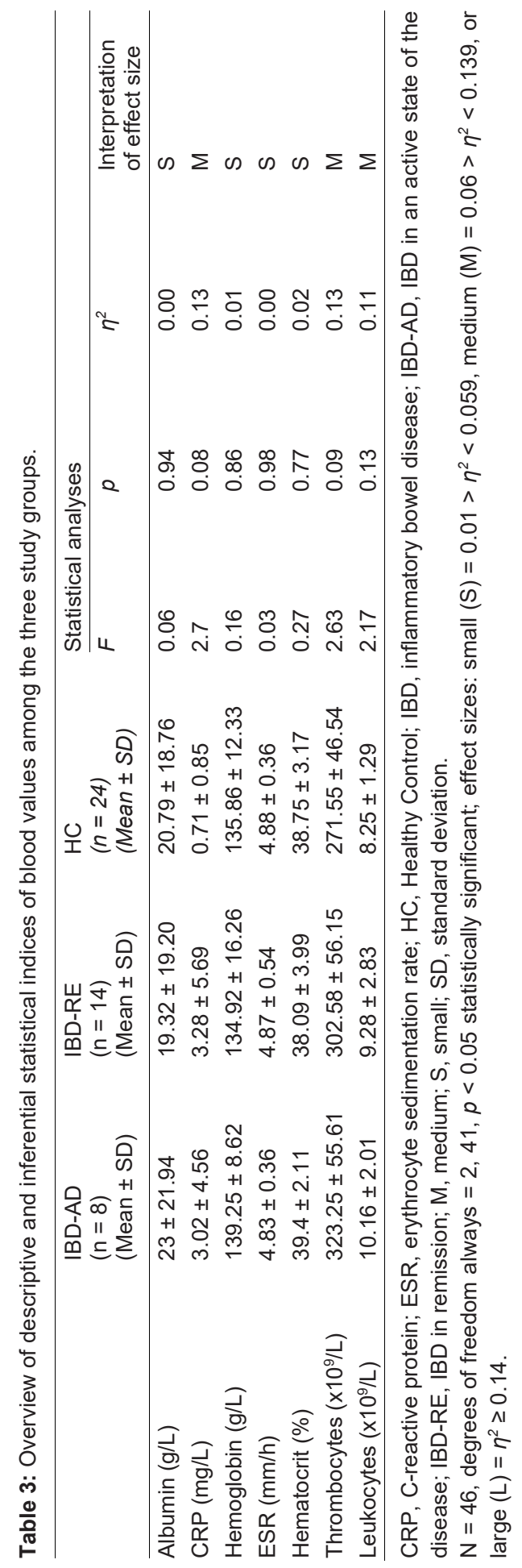


Correlation between subjective sleep and inflammation indices in participants with IBD

Table 4 gives the correlations of subjective sleep quality (as assessed by the ISI) with the inflammatory indices ESR and CRP. There were no significant correlations of subjective sleep with ESR or CRP in children and adolescents with IBD. However, the findings point to a large nonlinear correlation between ESR and ISI (inverse U-shape), meaning that with low and high values of ESR, subjective sleep complaints were low. At intermediate ESR values, subjective sleep complains peaked.

Correlation between objective sleep and inflammation in participants with IBD

Table 4 provides the correlational analyses results for objective sleep patterns and the inflammatory indices ESR and CRP among children and adolescents with IBD.

A statistically significant linear correlation was found between ESR and the percentage of stage 4 sleep ( $p=0.013$ ). Furthermore, a large linear correlation was found between ESR and time in stage 4 sleep (minutes).

No significant linear or nonlinear correlations were found between CRP and any of the subjective or objective sleep parameters. However, although not significant, the trend indicated a large nonlinear correlation between CRP and sleep latency (U-shape); this reflects higher sleep latency at both low and high values of CRP, and low sleep latency at intermediate levels of CRP. 
Table 4: Correlation coefficients between both subjective and objective sleep indices with inflammatory markers the C-reactive protein (CRP) and erythrocyte sedimentation rate (ESR).

\begin{tabular}{|c|c|c|c|c|c|}
\hline & & \multicolumn{4}{|c|}{ Inflammatory bowel disease } \\
\hline & & \multicolumn{2}{|c|}{ ERS $(\mathrm{mm} / \mathrm{h})$} & \multicolumn{2}{|c|}{$\mathrm{CRP}(\mathrm{mg} / \mathrm{L})$} \\
\hline & & $r$ & $r^{2}$ & $r$ & $r^{2}$ \\
\hline \multicolumn{2}{|c|}{ Sleep complaints Linear correlation } & 0.314 & 0.099 & 0.330 & 0.109 \\
\hline & Nonlinear correlation & 0.513 & 0.263 & 0.207 & 0.043 \\
\hline \multirow[t]{2}{*}{ Arousals } & Linear correlation & 0.073 & 0.005 & -0.038 & 0.001 \\
\hline & Nonlinear correlation & 0.138 & 0.019 & 0.084 & 0.007 \\
\hline \multirow[t]{2}{*}{ WASO (min) } & Linear correlation & 0.403 & 0.162 & 0.267 & 0.071 \\
\hline & Nonlinear correlation & 0.322 & 0.104 & 0.200 & 0.040 \\
\hline \multirow[t]{2}{*}{ Sleep latency } & Linear correlation & -0.054 & 0.003 & 0.397 & 0.158 \\
\hline & Nonlinear correlation & 0.346 & 0.120 & 0.838 & 0.702 \\
\hline \multirow[t]{2}{*}{ REM latency } & Linear correlation & -0.068 & 0.005 & -0.127 & 0.016 \\
\hline & Nonlinear correlation & 0.277 & 0.077 & 0.045 & 0.002 \\
\hline \multirow[t]{2}{*}{ Sleep duration } & Linear correlation & 0.120 & 0.014 & -0.017 & 0.000 \\
\hline & Nonlinear correlation & 0.184 & 0.034 & 0.045 & 0.002 \\
\hline \multirow[t]{2}{*}{ Sleep efficiency } & Linear correlation & -0.390 & 0.152 & -0.414 & 0.171 \\
\hline & Nonlinear correlation & 0.391 & 0.153 & 0.382 & 0.146 \\
\hline \multirow[t]{2}{*}{ REM (min) } & Linear correlation & 0.125 & 0.016 & -0.027 & 0.001 \\
\hline & Nonlinear correlation & 0.190 & 0.036 & 0.118 & 0.014 \\
\hline \multirow[t]{2}{*}{ REM (\%) } & Linear correlation & 0.027 & 0.001 & 0.042 & 0.002 \\
\hline & Nonlinear correlation & 0.084 & 0.007 & 0.245 & 0.060 \\
\hline \multirow[t]{2}{*}{ Stage $1(\min )$} & Linear correlation & 0.124 & 0.015 & 0.122 & 0.015 \\
\hline & Nonlinear correlation & 0.219 & 0.048 & 0.071 & 0.005 \\
\hline \multirow[t]{2}{*}{ Stage $1(\%)$} & Linear correlation & 0.109 & 0.012 & 0.115 & 0.013 \\
\hline & Nonlinear correlation & 0.321 & 0.103 & 0.084 & 0.007 \\
\hline \multirow[t]{2}{*}{ Stage 2 (min) } & Linear correlation & 0.041 & 0.002 & -0.072 & 0.005 \\
\hline & Nonlinear correlation & 0.130 & 0.017 & 0.173 & 0.030 \\
\hline \multirow[t]{2}{*}{ Stage $2(\%)$} & Linear correlation & -0.040 & 0.002 & -0.121 & 0.015 \\
\hline & Nonlinear correlation & 0.192 & 0.037 & 0.249 & 0.062 \\
\hline \multirow[t]{2}{*}{ Stage 3 (min) } & Linear correlation & -0.408 & 0.166 & -0.090 & 0.008 \\
\hline & Nonlinear correlation & 0.386 & 0.149 & 0.310 & 0.096 \\
\hline \multirow[t]{2}{*}{ Stage $3(\%)$} & Linear correlation & -0.448 & 0.201 & -0.052 & 0.003 \\
\hline & Nonlinear correlation & 0.392 & 0.154 & 0.326 & 0.106 \\
\hline \multirow[t]{2}{*}{ Stage 4 (min) } & Linear correlation & 0.439 & 0.192 & 0.113 & 0.013 \\
\hline & Nonlinear correlation & 0.671 & 0.450 & 0.479 & 0.229 \\
\hline \multirow[t]{2}{*}{ Stage $4(\%)$} & Linear correlation & $0.491^{*}$ & 0.241 & 0.203 & 0.041 \\
\hline & Nonlinear correlation & 0.566 & 0.320 & 0.436 & 0.190 \\
\hline
\end{tabular}

REM, rapid eye movement; WASO, wake after sleep onset.

$N=22$. Interpretation of correlation coefficients according to Cohen (1988) [23]: small: $|0.1|-|0.3|$; medium: $|0.3|-|0.5|$; large: $|0.5|-|1.0|$. Correlation is significant at the 0.05 level (one-tailed).

${ }^{*} p=0.013$. 


\section{Discussion}

In the present study, the following key findings were observed. First, subjective sleep did not descriptively or statistically differ between children and adolescents with IBD. Likewise, no differences were observed regarding the erythrocyte sedimentation rate (ESR), although descriptively CRP was higher in participants with IBD. Second, among participants with IBD, nonlinear associations between poor sleep and ESR and CRP were observed. Third and most strikingly, compared to healthy controls and pediatric patients with well-adjusted medication for IBD (IBD$\mathrm{RE}$ ), pediatric patients in an active state of disease (IBD-AD) had more unfavorable objective sleep markers such as longer periods of awakenings after sleep onset and a significantly shorter relative amount of time spend in stage 3 .

Four hypotheses were formulated, and each is considered now in turn.

Our first hypothesis was that children and adolescents with IBD-AD would report poorer subjective sleep than either those with IBD-RE or HCs, but our data only partially confirmed this hypothesis. Descriptively, but not statistically, IBDAD participants reported greater subjective sleep impairment than IBD-RE and HC participants, while subjective sleep in the IBD-RE group differed neither statistically nor descriptively from that of HC. In this view, the pattern of results is in line with previous studies $[9,16]$. Furthermore, in addition to studies by Pirinen et al. [11,16] and Ranjbaran et al. [9], Thompson et al. [24] reported that in the United States, adolescents diagnosed with CD (median age: 14 years, $53 \%$ boys; $\mathrm{N}=193$ ), disrupted sleep, fatigue, and worry about aches and pains correlated significantly and positively with IBD severity. Furthermore, Benhayon et al. [18] observed that in a US sample of pediatric CD patients with depression (mean age: 14.4 years, $62.5 \%$ female, $\mathrm{N}=115$ ), $53 \%$ had clinically significant sleep problems. However, we believe that the present findings expand upon previous results in that they were derived from a study of school children and participants on the verge of adolescence, while we also distinguished between participants with IBD in an active state of disease and those in remission, a distinction which, in our opinion, has been overlooked so far and which might explain the inconsistent and unclear findings on subjective sleep among these pediatric patients. However, on a subjective level, pediatric patients with IBD did not appear to report more impaired sleep than HCs. Furthermore, these results might help pediatric gastroenterologists to counsel both pediatric patients with IBD and their parents that, on a subjective level, sleep is not impaired.

Our second hypothesis was that objective sleep patterns (EEG recordings) would be poorer in children and adolescents with IBD-AD than in those with IBD$\mathrm{RE}$ or in $\mathrm{HCs}$, and this was confirmed. Thus, the present pattern is in line with those observed elsewhere, both in nonclinical samples of adolescents [7] and in middle-aged adults [7,8], and among adults with IBD [14]. However, the present 


\section{Chapter 4}

findings expand upon previous studies in identifying a more fine-grained pattern. First, the only sleep EEG study among patients with IBD involved a sample of adults; the only findings of note were shortened sleep duration and lower sleep efficiency in the patient groups than in HCs. We observed, among children and adolescents with active disease, a significantly increased time awake after sleep onset, a significant decrease in time spend in stage 3 , a greater frequency of arousals, a shorter sleep duration, and a lower sleep efficiency, in effect, both unfavorable sleep continuity and poorer sleep architecture. To our knowledge, this is the first study to show objectively poorer sleep dimensions among pediatric patients with IBD in an active state of disease than in pediatric patients in remission (IBD-RE) or healthy controls. However, the data available to us are unable to provide insight into the underlying physiological mechanisms. In the absence of such data, we speculate that the greater number of awakenings and the longer time awake after sleep onset might be due to disease-associated symptoms such as pain, stress, and depression as well as to medical treatment (corticosteroids) and disease-related immune changes [9]. Such sleep disturbances are of high importance in the regulation of several physiological processes involving the gastrointestinal tract $[25,26]$, and fragmented sleep can adversely affect normal functioning [27]. Furthermore, changes in circadian rhythm may affect normal gastrointestinal physiology and mucosal balance, either directly by altering normal molecular function, or indirectly through symptom-related disturbances [28]. Tang et al. [28] performed a study examining sleep deprivation in mice with colitis and noted that both acute and chronic sleep deprivation led to increased symptoms of colitis due to increased sensitivity to pro-inflammatory cytokines. Additionally, a large online survey among patients with CD in clinical remission showed that patients with subjective sleep disturbances had a twofold risk of active disease in the following six months [13]. Therefore, our findings, which are, to our knowledge, the first to be based on objective sleep in children and adolescents with IBD, confirm what has previously been found on the basis of subjective assessments. This is of considerable importance for clinical practice, since assessment of sleep, even subjectively, may serve as an indicator for objective sleep disturbances.

Our third hypothesis was that subjective sleep and acute and chronic inflammatory markers (CRP and ESR) would be related $[7,8,14]$, and this was partially confirmed. A nonlinear relationship was found between subjective poor sleep and markers of chronic inflammation (ESR) (inverse U-shaped), while rather lower and higher CRP values were associated with longer sleep onset latencies. These findings are broadly consistent with those from previous studies [8], although none of these studies described the relationship as nonlinear [16]. The underlying reason for sleep complaints increasing with higher markers for inflammation cannot be explained by the present data. Further, nonlinear computations are rarely 
reported, and it seems that typically linear associations are implicitly assumed. However, in one exception to this, Kaneita et al. [29] observed an inverse Ushaped association between very short sleep and very long sleep and subjective poor sleep among a large sample of Japanese adolescents. Likewise, Kalak et al. [26] reported a nonlinear association between subjective actual sleep duration and body mass index; that is, shorter and longer subjective actual sleep durations were associated with higher body mass index. Furthermore, Kalak et al. [26] found a nonlinear association between subjective actual sleep duration and feeling of being restored. In other words, both shorter and longer subjective actual sleep durations were associated with a lower feeling of being restored. To conclude, while with the present study we were able to show that nonlinear associations did indeed emerge between specific dimensions of sleep and inflammatory markers, we are unable to explain why this happened. Accordingly, we encourage further research groups to investigate whether and why nonlinear associations might occur.

Our fourth hypothesis was that objective sleep would be associated with acute and chronic inflammatory markers, and this was partially confirmed. Specifically, we expected that higher slow-wave sleep (SWS) indices would be associated with higher indices of immune regulation [30], and indeed, a significant association was found between stage 4 sleep (percentage and minutes) and ESR, while the correlation coefficient between stage 4 (percentage and minutes) and CRP was positive and thus in the expected direction.

The available data do not allow any deeper understanding of the mechanisms underlying the association between poor subjective and objective sleep, on one hand, and elevated markers of inflammation, on the other. However, on the basis of previous research [8,12,31-33] investigating the mechanisms linking sleep and immune system dynamics, we propose the following explanation: Immune challenges related to IBD result in nocturnal increases in proinflammatory cytokines [8] and cause an increase in symptoms, including nocturnal diarrhea, and changes in the sleepewake cycle as one of the first responses to acute inflammation [9]. Additionally, these symptoms might cause prolonged sleep latency and sleep disturbances and might interfere with the normal functioning of the sympathetic nervous system (SNS) and the hypothalamicepituitaryeadrenal (HPA) axis [34]. During normal nocturnal sleep, activation of the SNS would shift towards activation of the parasympathetic system, especially during non-rapid eye movement (NREM) and SWS sleep. However, during REM sleep and among patients with insomnia, more sympathetic activity is measured by plasma levels of sympathetic neurotransmitter and inflammation [8]. Nonetheless, it remains unclear whether increased SNS activation in disturbed sleep correlates with or mediates increases in inflammation, and a complex interaction might be at play. 


\section{Chapter 4}

A further possibility is that arousal due to physiological symptoms and the psychological burden of IBD may trigger HPA activation and therefore lead to disrupted sleep and disturbed cognitive-emotional processes $[35,36]$. According to the hyper-arousal model of insomnia advanced by Riemann et al. [35], psychological and physiological stressors lead to increased secretion of cortisol, which produces hypothalamicepituitaryeadrenocortical axis (HPAA) activation and disturbed sleep. Numerous studies have reported decreased psychological functioning on days following disturbed sleep and poor overall mental health in the long term $[11,17,37,38]$. Therefore, the interplay between neuroendocrine sleep regulation, psychosocial experience, and psychiatric difficulties seems to create a negative spiral leading towards the risk of chronic insomnia $[23,25]$.

Despite the novelty of our study, several limitations warrant caution against overgeneralization of the present findings. First, the relatively small sample, especially the small number of children in an active disease state, created difficulties in detecting statistically significant effects. However, we also focused on effect sizes, which are unrelated to sample sizes. Second, only participants willing and able to participate were assessed. Third, it was difficult to control for the possible influence of medication. Furthermore, it is conceivable that additional mediators, such as microbial or environmental conditions, influenced outcomes in the same or in opposite directions. Fourth, we did not distinguish between patients with $C D$ and UC; however, for diagnostic reasons, such an approach would have been easy to follow and to justify, we decided to split patients into IBD-AD and IBD-RE, as suggested by recent research such as that of Reigada et al. [16]. Finally, given the cross- sectional nature of the study, no conclusions can be drawn as to the direction of influence between disease activity or state of inflammation and sleep.

\section{Conclusion}

In children and adolescents with IBD, objective sleep disturbances such as significantly longer time awake after sleep onset, a significant decrease in the time spent in stage 3, increased frequency of arousals, shorter sleep duration, and lower sleep efficiency were observed. Furthermore, poor sleep and higher inflammation indices were associated in a complex manner; both linear and nonlinear associations were observed. Patterns of sleep and inflammations change as a function of the disease activity in IBD. It seems advisable to include the assessment of sleep quality in the care of pediatric IBD patients, since the assessment of sleep, even subjectively, may serve as an additional indicator for objective sleep disturbances and existing inflammation. 


\section{Acknowledgments}

The authors would like to thank the children and parents who agreed to participate in this study. We thank the Kantonsspital Aarau for providing additional recruitment options, as well as Marielle Koenig and Vladimir Djurdjevic for sleep EEG scoring. We are also grateful to Noe Stoll for support during data collection. Finally, we thank Nick Emler (Surrey, UK) for proofreading the manuscript. This work was supported by the Freiwillige Akademische Gesellschaft Basel (FAG, Basel, Switzerland).

\section{Conflict of interest}

The authors declare that they have no conflicts of interest in regard to this work.

The ICMJE Uniform Disclosure Form for Potential Conflicts of Interest associated with this article can be viewed by clicking on the following link: http://dx.doi.org/10.1016/ j.sleep.2017.08.015. 


\section{References}

1 Levine $\mathrm{A}$, de $\mathrm{Bie} \mathrm{Cl}$, Turner $\mathrm{D}$, et al. Atypical disease phenotypes in pediatric ulcerative colitis: 5-year analyses of the EUROKIDS registry. Inflamm Bowel Dis 2012:1e8.

2 Rabizadeh S, Dubinsky M. Update in pediatric inflammatory bowel disease. Rheum Dis Clin N Am 2013;39:789e99.

3 Loddo I, Romano C. Inflammatory bowel disease: genetics, epigenetics, and pathogenesis. Front Immunol 2015;6:551.

4 Dutta AK. Influence of environmental factors on the onset and course of inflammatory bowel disease. World J Gastroenterol 2016;22:1088.

5 Walter JG, Kahn SA, Noe JD, et al. Feeling fine: anxiety and depressive symptoms in youth with established IBD. Inflamm Bowel Dis 2016;22:402e8.

6 Rosen MJ, Dhawan A, Saeed SA. Inflammatory bowel disease in children and adolescents. JAMA Pediatr 2015;169:1053.

7 Fernandez-Mendoza J, Baker JH, Vgontzas AN, et al. Insomnia symptoms with objective short sleep duration are associated with systemic inflammation in adolescents. Brain Behav Immun 2017;61:110e6.

8 Irwin MR, Opp MR. Sleep health: reciprocal regulation of sleep and innate immunity. Neuropsychopharmacology 2017;42:129e55.

9 Ranjbaran Z, Keefer L, Farhadi A, et al. Impact of sleep disturbances in inflammatory bowel disease. J Gastroenterol Hepatol 2007;22:1748e53.

10 Legaki E, Gazouli M. Influence of environmental factors in the development of inflammatory bowel diseases. World J Gastrointest Pharmacol Ther 2016;7: 112e25.

11 Pirinen T, Kolho K-L, Ashorn M, et al. Sleep and emotional and behavioral symptoms in adolescents with inflammatory bowel disease. Sleep Disord 2014;2014:1e5.

12 Ali T, Choe J, Awab A, et al. Sleep, immunity and inflammation in gastroin- testinal disorders. World J Gastroenterol 2013;19:9231e9.

13 Ananthakrishnan AN, Long MD, Martin CF, et al. Sleep disturbance and risk of active disease in patients with Crohn's disease and ulcerative colitis. Clin Gastroenterol Hepatol 2013;11:965e71.

14 Keefer L, Stepanski EJ, Ranjbaran Z, et al. An initial report of sleep disturbance in inactive inflammatory bowel disease. J Clin Sleep Med 2006;2:409e16.

15 Werkstetter KJ, Ullrich J, Schatz SB, et al. Lean body mass, physical activity and quality of life in paediatric patients with inflammatory bowel disease and in healthy controls. J Crohns Colitis 2012;6:665e73.

16 Pirinen T, Kolho K-L, Simola P, et al. Parent and self-report of sleep-problems and daytime tiredness among adolescents with inflammatory bowel disease and their population-based controls. Sleep 2010;33:1487e93.

17 Brand S, Gerber M, Beck J, et al. Exercising, sleep-EEG patterns, and psychological functioning are related among adolescents. World J Biol Psychiatry 2010;11:129e40.

18 Benhayon D, Youk A, McCarthy FN, et al. Characterization of relations among sleep, inflammation, and psychiatric dysfunction in depressed youth with Crohn disease. J Pediatr Gastroenterol Nutr 2013;57:335e42.

19 Mikocka-Walus A, Knowles SR, Keefer L, et al. Controversies revisited: a systematic review of the comorbidity of depression and anxiety with inflammatory bowel diseases. Inflamm Bowel Dis 2016;22:752e62.

20 Bastien $\mathrm{CH}$, Vallieres A, Morin CM. Validation of the Insomnia Severity Index as an outcome measure for insomnia research. Sleep Med 2001;2:297e307.

21 Gerber M, Lang C, Lemola S, et al. Validation of the German version of the Insomnia Severity Index in adolescents, young adults and adult workers: results from three cross-sectional studies. BMC Psychiatry 2016;16:174. 
22 Rechtschaffen A, Kales A. A manual of standardized terminology, techniques and scoring system for sleep stages of human subjects. NIH Publication 204. Bethesda, MD: National Institutes of Health; 1968.

23 Cohen J. Statistical power analysis for the behavioral sciences. 2nd ed. Hill- sdale, NJ: Routledge; 1988.

24 Thompson RD, Craig AE, Mrakotsky C, et al. Using the Children's Depression Inventory in youth with inflammatory bowel disease: support for a physical illness-related factor. Compr Psychiatry 2012;53:1194e9.

25 James S, Hale L. Sleep duration and child well-being: a nonlinear association. J Clin Child Adolesc Psychol 2016;21:1e11.

26 Kalak N, Brand S, Beck J, et al. Association between subjective actual sleep duration, subjective sleep need, age, body mass index, and gender in a large sample of young adults. Neuropsychiatr Dis Treat 2015;11:107e13.

27 Turek FW, Joshu C, Kohsaka A, et al. Obesity and metabolic syndrome in circadian clock mutant mice. Science 2005;308:1043e5.

28 Tang Y, Preuss F, Turek FW, et al. Sleep deprivation worsens inflammation and delays recovery in a mouse model of colitis. Sleep Med 2009;10: 597e603.

29 Kaneita Y, Ohida T, Osaki Y, et al. Association between mental health status and sleep status among adolescents in Japan: a nationwide cross-sectional survey. J Clin Psychiatry 2007;68:1426e35.

30 Swanson GR, Burgess HJ, Keshavarzian A. Sleep disturbances and inflammatory bowel disease: a potential trigger for disease flare? Expert Rev Clin Immunol 2011;7:29e36.

31 Kinnucan JA, Rubin DT, Ali T. Sleep and inflammatory bowel disease: exploring the relationship between sleep disturbances and inflammation. Gastroenterol Hepatol 2013;9:718e27.

32 Grandner MA, Buxton OM, Jackson N, et al. Extreme sleep durations and increased C-reactive protein: effects of sex and ethnoracial group. Sleep 2013;36:769e79.

33 Shearer WT, Reuben JM, Mullington JM, et al. Soluble TNF-alpha receptor 1 and IL-6 plasma levels in humans subjected to the sleep deprivation model of spaceflight. J Allergy Clin Immunol 2001;107:165e70.

34 Irwin MR, Wang M, Campomayor $\mathrm{CO}$, et al. Sleep deprivation and activation of morning levels of cellular and genomic markers of inflammation. Arch Intern Med 2006;166:1756e62.

35 Riemann D, Spiegelhalder K, Feige B, et al. The hyperarousal model of insomnia: a review of the concept and its evidence. Sleep Med Rev 2010;14: 19 e31.

36 Baum KT, Desai A, Field J, et al. Sleep restriction worsens mood and emotion regulation in adolescents. J Child Psychol Psychiatry 2014;55:180e90.

37 Brand S, Kirov R, Kalak N, et al. Poor sleep is related to lower emotional competence among adolescents. Behav Sleep Med 2015:1e13.

38 Hatzinger M, Brand S, Perren S, et al. Pre-schoolers suffering from psychiatric disorders show increased cortisol secretion and poor sleep compared to healthy controls. J Psychiatr Res 2012;46:590e9. 



\section{Chapter 5}

\section{Aerobic exercise training in children and adolescents with inflammatory bowel disease: Influence on psychological functioning, sleep and physical performance - An exploratory trial}

Published as: MähImann L, Gerber M, Furlano RI, Legeret C, Kalak N, Sadeghi Bahmani D, et al.: Aerobic exercise training in children and adolescents with inflammatory bowel disease: Influence on psychological functioning, sleep and physical performance. Eur Psychiatry 2017;41:125. [IF: 2.21] 


\section{Chapter 5}

\section{Abstract}

People with Inflammatory Bowel Disease (IBD) are at risk for mental health issues, poor sleep quality and limited engagement in physical activity (PA). Standard treatment consists of immune modulating pharmaceuticals though evidence is growing that aerobic exercise training (AET) could serve as an adjuvant option to reduce disease symptoms and improve mental health. The aim of the present exploratory trial was to investigate possible AET effects on psychological functioning, symptoms of depression, sleep and physical activity in paediatric patients with IBD. Twenty-one paediatric patients with IBD and 23 gender- and agematched healthy controls $(\mathrm{HC})$ were assessed. The IBD group was further split into a "remission-group" (IBD-RE; $\mathrm{n}=14$ ) and an "active disease group" (IBD-AD; $\mathrm{n}=7$ ). All participants completed an 8-week AET exergame intervention reaching $60-80 \%$ of maximum heart rate for 5 days per week. At baseline and after 8 weeks, psychological functioning, depressive symptoms, objective sleep EEG, subjective sleep and objective and subjective PA were assessed. AET improved the exercise capacity of all participants. Self-reported fitness and daily physical activity increased in IBD-AD, but not in IBD-RE and HC. No improvements were observed for psychological functioning, depressive symptoms or subjective sleep. Objective sleep improved, deep sleep increased, light sleep decreased, and in the IBD-AD group the number of arousals after sleep onset decreased. Descriptively, the IBD-AD group reported lower psychological functioning and poorer subjective sleep quality. Among paediatric patients with IBD and healthy controls, an exergaming intervention has the potential to improve exercise capacity, self-reported fitness, daily physical activity, and aspects of objective sleep. Results suggest that children and adolescents in an active state of IBD are at increased risk of poor psychological functioning and poor sleep quality. 


\section{Introduction}

Inflammatory bowel diseases are among the most common chronic inflammatory diseases in the developed world and affect approximately $0.5-1.0 \%$ of the population during their lifetime (Russel, 2000). The term Inflammatory Bowel Disease (IBD) covers two major forms of chronic intestinal inflammation, Crohn's disease and ulcerative colitis, as well as atypical phenotypes (Levine et al., 2013). Exact causes are still unclear but seem to include a combination of immunological, genetic and environmental factors (Bishop, Lemberg, \& Day, 2014). The disease is characterized by recurrent cycles of remission and exacerbation.

In at least $20 \%$ of IBD patients, the disease is already apparent during childhood or adolescence, and this tendency is increasing, especially among Western populations (Russel, 2000). These young patients are confronted with the burden of lifelong disease management at an age when self-identity and psychosocial development is challenging (Mikocka-Walus, Knowles, Keefer, \& Graff, 2016). Unsurprisingly, adolescents with IBD frequently report discomfort and vulnerability, view themselves as different, and feel a loss of control over their lives and futures (Chouliaras et al., 2017; Nicholas et al., 2007).

As a result of the complex burden of disease, children and adolescents with IBD have an increased risk of displaying a broad variety of issues related to psychological functioning, sleep and inactivity. First, young people with IBD have a significantly increased vulnerability to psychiatric disorders when compared to healthy controls (Chouliaras et al., 2017; De Boer, Grootenhuis, Derkx, \& Last, 2005; Mackner \& Crandall, 2005; Vaisto, Aronen, Simola, Ashorn, \& Kolho, 2010). The rate of depression may be as high as $25 \%$, particularly among those with active IBD, a rate higher than for adolescents with other chronic diseases (Greenley et al., 2010; Mikocka-Walus et al., 2016). Therefore, the presence of severe IBD, accompanied by highly disruptive symptoms, increases psychological distress and the likelihood of depression. Second, sleep disturbances have been reported as a frequent issue in IBD (Kinnucan, Rubin, \& Ali, 2013; Werkstetter et al., 2012). While restoring sleep is strongly associated with physical, cognitive and psychological wellbeing, poor or disordered sleep is related to impairment of cognitive and psychological functioning and worsened physical health (Brand \& Kirov, 2011). Third, children and adolescents with IBD feel restricted and discouraged by their disease and tend to a sedentary lifestyle (Narula \& Fedorak, 2008; Werkstetter et al., 2012). Insufficient levels of physical activity (PA) result in maladaptation of the body, while sufficient PA has the potential to prevent and delay chronic disease risk factors and inflammatory processes (Booth, Roberts, \& Laye, 2012). In the context of IBD, current theories describe ways in which PA may have beneficial impacts on exercise capacity, anti-inflammatory processes, metabolic adaptions that may stimulate growth and development, mental 
health, stress management and sleep quality (Bilski, Mazur-Bialy, Wierdak, \& Brzozowski, 2013).

The current first-line treatment for IBD consists of monoclonal antibody therapy directed against the tumor necrosis factor-alpha (anti-TNFs) (Jossen \& Dubinsky, 2016; Scott \& Lichtenstein, 2016). Psychotherapeutic interventions seem to have the potential to reduce symptoms of depression among paediatric patients with IBD (Keerthy et al., 2016), though no evidence has so far been found for benefits of psychological and psychotherapeutic interventions in adult patients with IBD (Timmer et al., 2011). Another line of treatment involves regular exercising.

As regards the influence of physical activity interventions on patients with IBD, so far two systematic reviews have been published (Narula \& Fedorak, 2008; Packer, Hoffman-Goetz, \& Ward, 2010), from which five unique papers were identified (D'Inca et al., 1999; Elsenbruch et al., 2005; Gupta, Khera, Vempati, Sharma, \& Bijlani, 2006; Loudon, Corroll, Butcher, Rawsthorne, \& Bernstein, 1999; Ng et al., 2015). All of these studies were case-control studies in adult populations investigating the effect of moderate cycling (D'Inca et al., 1999), lowintensity walking (Loudon et al., 1999; Ng et al., 2015), yoga (Gupta et al., 2006) and an intervention including stress management training, moderate exercise, Mediterranean diet, behavioral techniques and self-care strategies (Elsenbruch et al., 2005). Compared to control conditions, interventions led to stress reduction and to subjective improvements in symptoms. Further, exercise had the potential to improve subjective quality of life, while the impact on immune markers remained unclear. Next, in line with previous findings, there have been two additional intervention studies with adult patients (Gerbarg et al., 2015; Klare et al., 2015). Klare et al. (2015) compared the influence on adult patients with IBD of a 10-week low-intensity physical activity-program (three times per week) with a control condition and observed improvements in health-related quality of life in both conditions, while no changes in biomarkers such as inflammatory markers were observed. Gerbarg et al. (2015) investigated the impact on adult patients with IBD of a 26-week Breath-Body-Mind workshop as compared to a control condition. The intervention included breathing techniques, far-East meditation and movement techniques (e.g., Aikido, Qigong), and regular exercising at home. Of particular note, the control condition involved an educational seminar; thus, group interaction, time spent with the study staff, and expectations were equivalent across conditions. The main results were that, compared to the control condition, symptoms of anxiety, depression, and perceived stress, and inflammatory markers decreased in the intervention condition.

Finally, Werkstetter et al. (2012) compared a paediatric sample of 39 six to twenty year olds with IBD ( $n=26$ in remission; $n=13$ in a mild active disease) to 39 gender- and aged-matched controls and observed that, compared to controls, 
participants with IBD had a lower grip strength and a shorter mean duration of physical activity per week; however, these differences were specific to female patients with mild disease. No differences were found for quality of life, sleep duration, or energy intake.

To summarize, from the two reviews (Narula \& Fedorak, 2008; Packer et al., 2010) and the two recent studies (Gerbarg et al., 2015; Klare et al., 2015) it turned out that these studies have involved only adult patients with IBD in either a remitted or mildly active state of disease. Further, control conditions with one exception (Gerbarg et al., 2015) have not controlled for social interactions, time spent with the study staff, or expectations. Last, sleep has been an outcome variable and subjectively assessed in only one study. Most importantly, no intervention and case-control research was performed among children and adolescents with IBD. This is astonishing, as in about $20 \%$ of IBD patients, the illness emerges already during childhood. The only case examining physical activity among children and adolescents was a comparative cross-sectional study by Werkstetter et al. (2012). Accordingly, with the present exploratory trial, study we aimed to fill these gaps. We assessed children and adolescents with IBD in both an active and a remitted state; we undertook an intervention study examining the effect of physical activity (more specifically: exergaming); the control condition was such to exclude methodological biases; we assessed psychological functioning, sleep (both subjectively and objectively), physical activity performance, and exercise capacity.

Several studies have reported a positive influence of physical activity on both subjective and objective sleep among healthy adolescents and adults (Chennaoui, Arnal, Sauvet, \& Leger, 2015; Kalak et al., 2012; Lang et al., 2013, 2016), but there is no equivalent study involving paediatric patients with IBD. Accordingly, a further aim of the present exploratory trial was to shed more light on this issue.

Given the encouraging results obtained for adult patients with IBD, the following four exploratory research questions were formulated. First, on the basis of previous findings (Gerbarg et al., 2015; Josefsson, Lindwall, \& Archer, 2014; Kalak et al., 2012; Narula \& Fedorak, 2008; Packer et al., 2010), we investigated whether poor psychological functioning and depressive symptoms decreased after 8 weeks of aerobic exercise training (AET). Second, following others (Kalak et al., 2012; Lang et al., 2013, 2016), we examined whether sleep quality improved after the AET intervention. Third, following Sween et al. (2014), we explored whether the AET had a beneficial effect of AET on subjectively and objectively assessed physical activity over the course of the intervention. Finally, following Maddison et al. (2012), we explored whether exercise capacity improved after 8 weeks of AET. 


\section{Materials and methods}

\section{Study design and procedure}

Paediatric patients with IBD were recruited from the University Children's Hospital Basel (Switzerland). Eligible patients were informed about the study aims and confidentiality. The participants were invited to the hospital for baseline assessment, and anthropometric and demographic data were collected. At baseline as well as after completion of the 8-week AET, participants completed questionnaires covering psychological functioning, depressive symptoms, subjective sleep and physical activity. They performed a fitness test and wore an accelerometer device during the first and last weeks of the intervention. Finally, sleep architecture and continuity was assessed at home, the night before a regular school day using sleep-electroencephalography (sleep-EEG).

The exploratory trial was approved by the local ethical committee (EKNZ: 2014:220) and was conducted in accordance with the ethical principles laid down in the Declaration of Helsinki (Trial registration number: NCT02264275).

\section{Sample}

A total of 31 eligible children and adolescents with IBD were approached. Of these, 29 (93.5\%) agreed to participate in the study, though five subsequently withdrew from the study because of time constraints, and three withdraw due to acute illness (flu). The final sample consisted of 21 children and adolescents with IBD $(67.7 \%$ of the sample approached; mean age: 13.88 years; females, $n=10$ [43.5\%]). It included seven participants with an active disease state and 14 in remission, according to the regularly assessed PUCAI (Paediatric ulcerative colitis activity index) scores and PCDAI (Paediatric Crohn's disease activity index) (clinical scores) (Turner et al., 2010). Inclusion criteria were: (1) aged 6-20 years; (2) clinically and histologically confirmed diagnosis of IBD; (3) willing and able to take part in the study; (4) able to communicate and to complete questionnaires in German; (5) having a television at home; (6) written informed consent from both the children/adolescents and their parents (or a legal caregiver). Exclusion criteria were: (1) severe physical diseases of the locomotor apparatus; (2) psychiatric disorders such as psychotic disorders; severe affective disorders, eating disorders, mental retardation, autism spectrum disorder; (3) unable to communicate and to complete questionnaires in German; (4) among female adolescents: pregnancy, breastfeeding, or intention to get pregnant during the study period; (5) refusal to give written informed consent; (6) no television at home.

In parallel, 23 age- and gender-matched healthy controls (mean age: 12.38 years \pm 3.24 , females: $n=15[62.5 \%]$ ) were recruited by word of mouth recommendations. Inclusion criteria and exclusion criteria for healthy controls were the 
same as for patients with IBD except that they had to be physically and psychologically healthy.

Note that healthy controls underwent the same physical activity intervention program as paediatric patients with IBD. Thus, there was no non-intervention condition.

Table 1 reports all descriptive and statistical indices for the anthropometric measures comparing the three groups, namely active disease state patients (IBD$A D)$, patients in remission (IBD-RE) and healthy controls (HC) at baseline.

Table 1: Sample characteristics.

\begin{tabular}{llll}
\hline & IBD-AD & IBD-RE & $\mathrm{HC}$ \\
& $\mathrm{N}=7$ & $\mathrm{~N}=14$ & $\mathrm{~N}=23$ \\
& $\mathrm{M}(\mathrm{Cl})$ & $\mathrm{M}(\mathrm{Cl})$ & $\mathrm{M}(\mathrm{Cl})$ \\
\hline Age (years) & 14.69 & 13.23 & 12.38 \\
Female & $4(50 \%)$ & $6(42.9 \%)$ & $15(62.5 \%)$ \\
IBD & & & \\
Colitis ulcerosa & $3(37.5 \%)$ & $4(28.6 \%)$ & \\
Crohn's disease & $5(62.5 \%)$ & $7(50 \%)$ & \\
Undefined colitis & & $3(21.4 \%)$ & \\
Time since diagnosis & $111.5(41.86 ; 181.23)$ & $124.74(71.45 ; 178.04)$ \\
(months) & & & \\
Waist circumference $(\mathrm{cm})$ & $67.63(60.07 ; 75.19)$ & $65.86(61.86 ; 69.86)$ & $64.82(61.4 ; 68.24)$ \\
Difference from norm & $-9.81(-14.95 ;-4.68)$ & $-8.89(-11.67 ; 6.10)$ & $-8.868(-11.29 ;-6.44)$ \\
Height (cm) & $153.48(138.82 ;$ & $153.57(146.64 ;$ & $152.65(145.33 ;$ \\
& $168.13)$ & $160.51)$ & $159.97)$ \\
Weight (kg) & $45.28(32.03 ; 58.52)$ & $44.043(36.43 ; 51.66)$ & $44.79(38.37 ; 51.22)$ \\
BMI (kg/m $\left.{ }^{2}\right)$ & $18.6(15.78 ; 21.42)$ & $18.21(16.54 ; 19.89)$ & $18.46(17.17 ; 19.76)$ \\
BMI z-score & $-0.59(-1.25 ; 0.07)$ & $-0.34(-0.9146 ; 0.2315)$ & $-0.18(-0.54 ; 0.17))$ \\
Percentile BMI vs age & $31.98(11.65 ; 52.30)$ & $39.82(23.972 ; 55.674)$ & $44.23(33.52 ; 54.93)$ \\
Z-score height vs age & $-0.91(-2.36 ; 0.53)$ & $0.022(-0.5 ; 0.54)$ & $0.12(-0.32 ; 0.55)$ \\
Percentile height vs age & $32.04(4.46 ; 59.62)$ & $52.91(37.37 ; 68.44)$ & $52.86(39.5 ; 66.23)$ \\
\hline
\end{tabular}

* Notes: $\mathrm{N}=44$, all data presented as means. 95\% confidence intervals are shown in parentheses; IBD-AD: IBD in an active state of the disease; IBD-RE: IBD in remission; , HC: Healthy Control.

\section{Instruments}

\section{Assessment of psychological functioning}

The KIDSCREEN 27 (Ravens-Sieberer et al., 2008) consists of 27 items covering the following five domains: physical wellbeing, psychological wellbeing, autonomy and relations with parents, social support and peers, school environment. Answers are given on a 5-point rating scale $(1=$ not at all, $5=$ extremely/always $)$ and summed with higher scores reflecting better psychological functioning. 


\section{Chapter 5}

Validity and reliability of this instrument has been reported by Ravens-Sieberer et al. (2008) (Cronbach's alpha $=0.74$ to 0.86 ). The Child Depression Screener (ChilD-S) is a self-report screening instrument for symptoms of depression and consists of 8 items covering participants' feelings during the last two weeks (e.g. "I am not happy", "I feel exhausted") with ratings on 4-point Likert scales, ranging from 0 (true) to 3 (not true); higher values reflecting more marked depressive symptoms. Total scores were summed. The cut-off value of 11 points towards clinically relevant depression. Validity and reliability of the ChilD-S has been demonstrated previously (Fruhe et al., 2012) (Cronbach's alpha $=0.88$ ).

\section{Assessment of sleep}

To assess sleep objectively, sleep EEG recordings were performed at home using a one-channel EEG device (Fp2-A1, electrooculogram; electromyogram; Somnowatch; Randersacker, Germany). Two experienced raters visually analyzed sleep polygraphs according to standard procedures (Rechtschaffen \& Kales, 1968).

The device provided assessment of total sleep time, sleep efficiency, sleep onset latency, stages 1-4 (minutes and percentage), light sleep (stages 1 and 2), slow wave sleep (stages 3 and 4), rapid eye movement (REM) sleep, and number and times of awakenings after sleep onset.

To assess subjective sleep quality: the Insomnia Severity Index (ISI) was applied (Bastien, Vallieres, \& Morin, 2001). The tool consists of seven items, answered on 5-point rating scales ranging from 0 (not at all) to 4 (very much), (e.g., "difficulty in falling asleep", and "worrying about sleep"). The higher the overall score, the more the respondent is assumed to suffer from sleep disturbances. Validity of this instrument has been established for adolescents (Gerber et al., 2016) (Cronbach's alpha $=0.88)$.

\section{Assessment of subjective and objective physical activity}

To estimate the subjective level of physical activity (PA) and sedentary behaviour the short form of the International Physical Activity Questionnaire (IPAQ-S) questionnaire was applied as an internationally approved instrument (Craig et al., 2003; Ottevaere et al., 2011). The IPAQ-S assesses self-reported vigorous- and moderate-intensity PA, walking, total PA and time spent sitting over the past seven days. Where minutes per intensity exceeded 180 per day, the values were truncated to a maximum of 180 min per day. Data were reported in hours per day and summed using standardized IPAQ scoring protocols to derive total metabolic equivalent minutes (MET-minutes) of PA per week. Total MET-minutes per week was computed by the following formula: 
[Walking MET-minutes $/$ week $=3.3$ * walking minutes * walking days]

+ Moderate MET-minutes $/$ week $=4.0$

* moderate-intensity activity minutes * moderate days]

+ Vigorous MET-minutes $/$ week $=8.0$

* vigorous-intensity activity minutes * vigorous-intensity days]

= Total PA MET-minutes / week.

Based on the activity level, three metabolic equivalent task categories (MET Cat) were created: MET Cat 1 (inactive) are those who reported no activity or some activity, but not enough to meet categories 2 or 3 . MET Cat 2 (minimally active) is anyone who is either three or more days vigorously active for at least $20 \mathrm{~min}$ per day, or five or more days of moderate-intensity activity or walking for at least 30 min per day, or five or more days of any combination of walking, moderateintensity or vigorous intensity activities achieving a minimum of at least $600 \mathrm{MET}$ $\mathrm{min} /$ week. MET Cat 3 is anybody who is either vigorously active on at least three days and accumulating at least 1500 MET minutes a week, or seven or more days of any combination of walking, moderate intensity or vigorous intensity activities achieving a minimum of at least 3000 MET minutes/week. Sitting on weekdays and weekend days was assessed in hours.

Self-reported fitness was assessed on a 5-point Likert scale and validity of the instrument has been verified for adolescents by Hagstromer et al. (2008). Time participants spend sedentary was assessed in hours for the past seven days.

To assess physical activity objectively, we used the Fitbit-Flex® (Fitbit Inc., San Francisco, CA, USA) wristband accelerometer with three-dimensional motion sensing technology to measure movement $24 \mathrm{~h}$ a day, making a continuous assessment for five days (two weekend days and three weekdays). Fitbit data were recorded using the 1-min epoch setting and downloaded from the user website via the device's USB docking port in a raw data format. Validation studies have been conducted with children and adolescents (Meltzer, Hiruma, Avis, MontgomeryDowns, \& Valentin, 2015). The outcome variable was steps per day. The tool also allowed monitoring of adherence to the treatment regimen.

\section{Assessment of exercise capacity}

To assess functional exercise capacity, the submaximal 6-min walking test $(6 \mathrm{MWT})$ was used. Distance walked during 6 min was assessed and compared to reference age and gender norms (Ulrich et al., 2013). The 6MWT is well-standardized and increasingly utilized in pediatric populations with chronic diseases (Hassan, van der Net, Helders, Prakken, \& Takken, 2010). Heart rate (HR) was continuously recorded during the test with a heart rate sensor (POLAR®). 


\section{Chapter 5}

Perceived breathlessness was measured with the Modified Borg Rating of Perceived Exertion (RPE) scale, with scores ranging from 0 (no perceived breathlessness) to 10 points (maximum perception of breathlessness) (Borg, 1998).

\section{Intervention}

The AET intervention incorporated an active video gameplay, also called exergame. The exergame approach combined various behavior change techniques: First of all, exergames have been selected as easily accessible intervention with adjustable levels of intensity to minimalize perceived barriers, perceived threads and as an intervention to facilitate action taking in a safe environment. Second, gamification, as the application of design elements in order to engage and motivate players, was applied. Gamification techniques and mechanisms enable to achieve goals, encourage players through rewards and points, while offering intrinsic motivation in the form of fun (Schoech, Boyas, Black, \& Elias-Lambert, 2013). Third, self-efficacy (Bandura, 1977) was targeted as the feeling of confidence in one's own ability to achieve intended results. In the exergame context, this can be translated in the ability to earn points in the game, which provides children with the sense of accomplishment. This increasing self-efficacy might lead to increased levels of personal efficacy including coping behavior, action taking and sustaining in the face of obstacles and aversive experiences. Fourth, and closely related, through the visualization of feedback and success after each single round of playing the exergame, as well as the overview of points for longer time periods, increased motivation and intervention adherence is expected.

After the review of various exergames and their intensity levels, we decided to use the Just Dance Kids $₫$ and Sports-Resort $\circledast$ exergame for Nintendo Wii $\circledast$ in the present study. Just Dance Kids $®$ is a motion-based dancing game offering the player a collection of songs with accompanied dance choreography. During the songs, players mirror the dance performance and comments displayed on the screen, and are awarded for their accuracy. In addition, gold moves are integrated, which result in bonus points if mirrored correctly. The Nintendo Wii SportsResorts ${ }^{\circledR}$ game allows children to participate in a sports game in a virtual world. Players can choose between sward play, wakeboarding, frisbee, archery, basketball, table tennis, golf, bowling, canoeing and cycling. Players can either compete against the video game or can play against a second player. Children and adolescents were offered to choose one of the games and were encouraged to use the exergames for $30 \mathrm{~min}$, five days a week for a total of eight weeks, reaching a heart rate intensity of between 60 and $80 \%$ of maximum (based on the commonly used 220-age formula) (Tanaka, Monahan, \& Seals, 2001). Participant's parents were asked to monitor the intervention frequency in a paper diary to keep track of intervention adherence over the entire intervention period. 


\section{Statistical analysis}

The statistical assessment comprised a summary of descriptive statistics including means, confidence intervals and percentages. Diagnostic clusters (IBD-AD vs IBD-RE vs HC) were compared and effect sizes were reported. Effect sizes were reported as partial eta-squared $\left[\eta^{2}\right]$ and interpreted as follows: small $(\mathrm{S})=$ $.0012>\eta^{2}<.059$, medium $(\mathrm{M})=.06>\eta^{2}<.139$, or large $(\mathrm{L})=\eta^{2} \geq .14$. [23]. SPSS 22.0 (IBM Corporation, Armonk NY, USA) for Windows $®$ was used for all statistical analysis.

\section{Results}

\section{Intervention fidelity}

All participants were able to regularly execute the intervention. Further, parents and children reported that the Nintendo $\mathrm{Wii} \circledast$ was an attractive and innovative exergaming tool. Based on the activity diaries, adherence of the intervention playing 5 times per week for 8 consecutive weeks was accomplished by IBD-AD with $77.5 \%$, IBD-RE with $84.5 \%$ and $\mathrm{HC}$ with $88.7 \%$.

\section{Psychological functioning}

Table 2 provides an overview of the descriptive statistics for psychological functioning and depressive symptoms. Further, effect sizes were represented separately by time (before vs after intervention), group (IBD-AD vs IBD-RE vs HC), and interaction (group $x$ time). Descriptively, IBD-AD scored lower in the physical wellbeing and psychological wellbeing as compared to IBD-RE and HC during pre-assessment. There were no changes over time on any of the dimensions, namely physical wellbeing, psychological wellbeing, autonomy and parent relation, social support and peers, school environment or depressive symptoms. No group effects were observed for physical wellbeing, autonomy and parent relation, social support, peers, school environment or depressive symptoms. A medium effect size indicated lower physical wellbeing and higher scores on the depression screener in the IBD-AD group than in IBD-RE and HC. Finally, no time group interaction effects were observed for any subjectively assessed dimension of psychological functioning or depressive symptoms.

\section{Sleep}

Table 3 sets out the descriptive statistics for objective and subjective sleep as outcome variables. Further, effect sizes were represented separately by time 


\section{Chapter 5}

(before vs after intervention), group (IBD-AD vs IBD-RE vs HC), and interaction (group $x$ time).

\section{Objective sleep-EEG}

Time effects were found for REM, Stage 1 and Stage 4 sleep, showing that bed times and Stage 4 sleep increased, whereas REM and Stage 1 sleep decreased over time from pre- to post-intervention. There were no significant time effects for arousal, wake after sleep onset (WASO) min, SOL, REM latency, sleep duration, sleep efficiency, Stage 1 (\%), Stage 2 (min, \%), Stage 3 (min, \%) or Stage 4 (min).

A moderate time effect was observed only for arousal, sleep duration and Stage 4 ( $\mathrm{min}$ ), while all other parameters showed only small effect sizes. For the number of arousals, in the IBD-AD arousals diminished from 17 to 12 times per night, in IBD-RE these remained constant with 13 arousals per night and in $\mathrm{HC}$ arousals diminished from 14 to 12 . Concerning sleep duration, IBD-AD showed a decrease from 7:33 to 6:38 h per night; IBD-RE and $\mathrm{HC}$ remained constant with 8:10 and 8:00 $\mathrm{h}$ respectively over the pre-test night and 8:09 and 8:15 $\mathrm{h}$ over the post-test night. Stage $4(\mathrm{~min})$ increased in all three groups, IBD-AD, IBD-RE and $\mathrm{HC}$, by 7,11 and 5 min respectively.

Between-group differences were found for wake time after sleep onset (WASO) (min), sleep duration, and Stage 3 sleep ( $\mathrm{min}, \%)$. With regard to effect sizes, there were medium group effects for REM minutes and Stage 2 (min), which were lowest in the IBD-AD, and Stage 4 (\%), which was highest in the IBDAD group.

Concerning time group interaction effects, there were no interactions for any of the variables. Regarding the effect sizes, a moderate group by time interaction was found for REM latency, sleep duration and REM sleep (min, \%). REM latency remained stable among IBD-AD, increased among IBD-RE and decreased among HC. Sleep duration decreased by almost $1 \mathrm{~h}$ among IBD-AD, but was unchanged among IBD-RE and HC. REM sleep (min, \%) decreased for IBD-AD and IBD-RE, whereas no changes occurred in HC. Finally, minutes spent in Stage 2 sleep decreased for IBD-AD, whereas an increase was found for IBD-RE and $\mathrm{HC}$. 


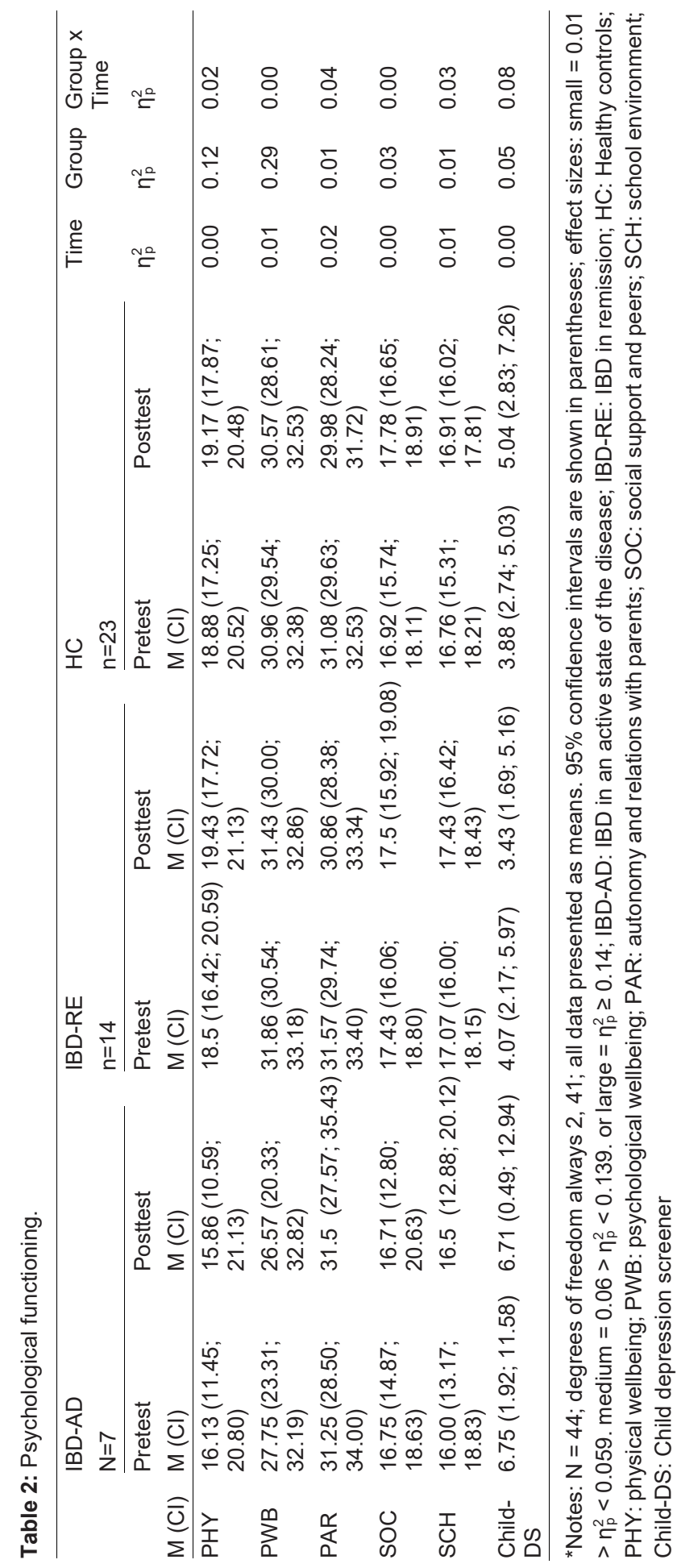




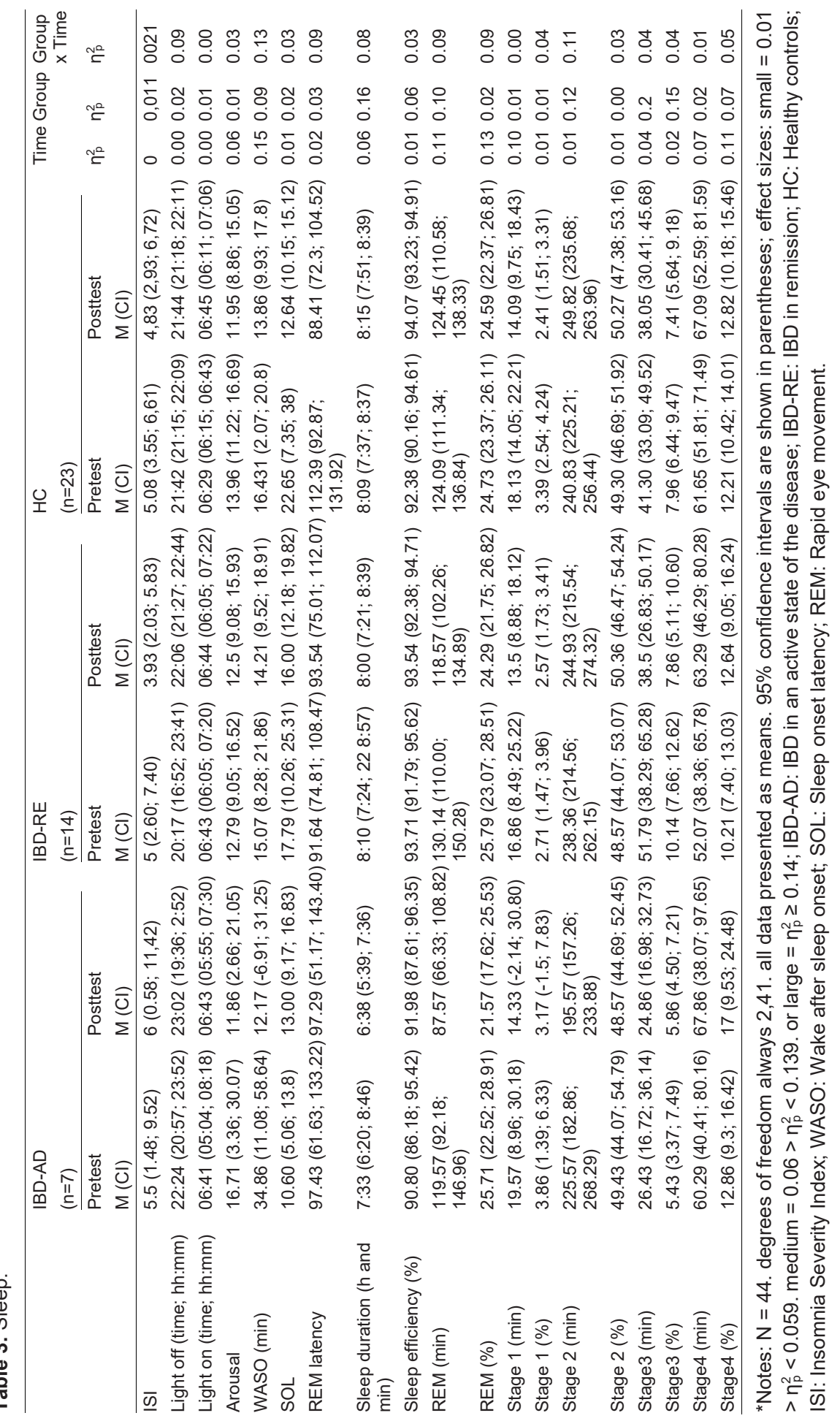




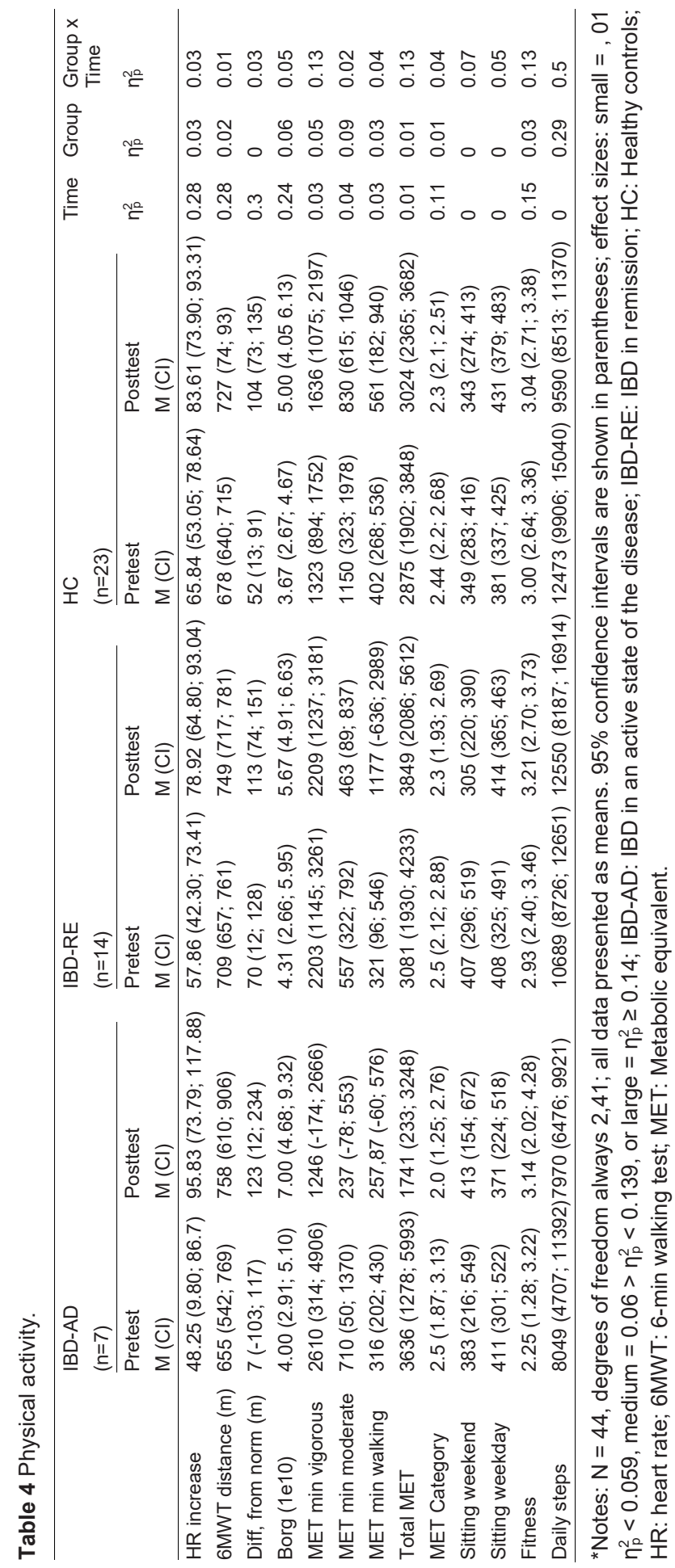




\section{Chapter 5}

Subjective sleep

The analysis of subjective sleep quality did not reveal any differences over time or between the three groups. Moreover, no group by time interaction was observed.

Physical activity

Table 4 provides the descriptive statistics for the subjective and objective measures of physical activity (PA) and exercise capacity.

Over time, the MET Cat decreased across all groups, which indicates reduced self-reported daily PA (Fig. 1). No time effects were observed for vigorous PA (MET), moderate PA (MET), walking (MET), total PA (MET), sitting during weekends and weekdays, or step counts.

Comparing groups, MET for vigorous activity was lower in IBD-AD than in IBDRE or HC. No between-group differences were observed for METs resulting from moderate PA, walking, total PA, sitting on weekdays and weekends, or self-reported fitness. Eta-square values indicated large effect size for step counts.

Group time interactions were identified for total MET, with decreases in IBD$A D$, increase in IBD-RE and stable scores in HC. The partial eta-squares values indicated that the effects were of moderate magnitude. Moreover, a group time effect reflected increased sitting time over the weekend and step counts in IBDAD but decreases in IBD-RE and HC.

With respect to self-reported fitness, scores increased in IBD-AD and IBD-RE, but remained stable in $\mathrm{HC}$, as indicated by a moderate effect size (Fig. 2).

\section{Exercise capacity}

Over time, increases were observed for heart rate (HR) and 6MWT distance in all groups, which differed from age and gender-based norms. Further, self-perceived intensity of the 6MWT increased from pre-to post-intervention in all groups.

No between-group differences were identified with regard to heart rate, 6MWT distance, difference from age- and gender-based norms, ratings on the RPE scale, or step counts.

Group time interactions for step count were observed (Fig. 3), with higher step counts in the IBD-AD as compared to the HC.

\section{Discussion}

The key findings of the present study were that an exergaming intervention involving $30 \mathrm{~min}$ of activity for five days a week over eight consecutive weeks improved exercise capacity across all participants (paediatric patients with IBD and healthy controls), while self-reported fitness and daily physical activity increased 
among IBD patients with active disease (IBD-AD). Furthermore, IBD-AD status was associated descriptively with lower psychological functioning and poorer sleep quality, which did not improve over of the period of the intervention. Next, the AET intervention led to better objective sleep parameters such as increased deep sleep (S 4) and decreased light sleep (S 1). Most importantly, the number of arousals after sleep onset decreased in the IBD-AD condition, suggesting more stable sleep continuity.

Four research questions were formulated and each of these will now be considered.

With the first research question, we investigated whether AET impacted positively on psychological functioning and depressive symptoms; this question has to be denied. There were no improvements either with regard to depressive symptoms or the dimensions assessed with the KIDSCREEN-27. The lack of impact of AET on psychological functioning and depressive symptoms is in marked contrast to many studies demonstrating a positive influence of structured exercise training on mental health both in general and specifically for adult patients with IBD (Gerbarg et al., 2015; Klare et al., 2015; Narula \& Fedorak, 2008; Ng et al., 2015; Packer et al., 2010). The following possible reasons can be advanced for the non-significant findings. First, the majority of our sample of patients could be characterized as mentally healthy and consequently both from a practical and statistical point of view improvements were unlikely. Consistent with this, high pretest values provided only limited scope for improvement (Wanrooij, Willeboordse, Dompeling, \& van de Kant, 2014). Second, as Stanton and Reaburn (2014) concluded from their review, exercise needs to be sustained in the longer term to affect mental health. Therefore, it seems likely that only a prolonged period of exercising may lead to long-term benefits for mental health. Put another way, the intervention period of eight weeks might have been too short or at too low intensity. Third, a control condition might have allowed for detection of potential improvements. Fourth, self-perception of psychological functioning may have changed, but in ways too subtle to be captured by the questionnaires, which might have been inappropriate or too coarse-grained. Fifth, previous demonstrations of the exercise effect have been exclusively derived from studies with adult patients with IBD, but also with different sample sizes and, most importantly, with different methodological approaches and interventions (Gerbarg et al., 2015; Klare et al., 2015; Narula \& Fedorak, 2008; Packer et al., 2010). Thus, the comparison with previous studies is difficult. Sixth, most importantly, as regards the sample characteristics, in the present study the remitted versus active disease-ratio was 2:1, while in other studies, such ratio information was not clearly provided. Accordingly, a direct comparison of the outcome results seems methodologically difficult.

With the second research question we examined whether there was a positive influence of AET on sleep patterns. While this research question has to be denied 
as regards subjective sleep, specific favorable sleep changes were observed such as increased deep sleep (S 4), decreased light sleep (S 1), and, most impressively, fewer arousals were observed in the IBD-AD condition. Collectively, such positive changes are in accord with previous results (Chennaoui et al., 2015; Kalak et al., 2012; Lang et al., 2013, 2016). The present study adds to the current literature in a further important way in that we were able to show that the IBD-AD group had negative outcomes with respect to wake time after sleep onset, sleep duration and deep sleep sequences as compared to both other groups. On the other hand, and again as regards the IBD-AD group, the available data do not explain why objective sleep duration decreased, which was against expectations. Specifically, as reported in Table 3, we observed that, compared to baseline, participants in the IBD-AD group slept about $1 \mathrm{~h}$ less, started to sleep about $1 \mathrm{~h}$ later and had a longer SOL, along with a lower sleep efficiency.

Other sleep indices such as WASO and duration and percentage of stages (14 ) did not change. Therefore, it appears that, on the basis of some sleep continuity indices (behalve the number of arousals as a contrary and positive change), sleep decreased, but not on the basis of sleep architecture indices. In the absence of further data, we offer the following explanation. It is possible that the second assessment occurred during a stage of flare-up. Underlying reasons for the deviation of the present findings from expectation cannot be found in the evidence available to us.

With the third research question, following Sween et al. (2014), we explored whether there would be a positive effect of AET on subjective and objective physical activity, and this was partially supported. Whereas objectively assessed daily step counts significantly improved in the IBD-AD group over the intervention period, no improvements were observed in the other groups. These findings are in line with a recent review summarizing 27 studies (Peng, Lin, \& Crouse, 2011), all of which reported a positive benefit of active video gaming on exercise expenditure and its ability to meet national physical activity guidelines. This might be explained by the fact that patients in an active state of the disease were quite inactive at baseline and thus had more potential and increased motivation for improvement. The present findings therefore confirm the beneficial effects of PA interventions using exergames to significantly increase PA or step counts for the IBD-AD group (Peng et al., 2011).

Our fourth research question, following Maddison et al. (2012), explored whether there would be improvement in exercise capacity, and this was supported. All three groups achieved a significantly increased distance in the 6MWT, while increments in heart rate and estimates of perceived exertion were also found. This pattern of results indicated an increase in exercise capacity and cardiovascular fitness and confirms previous research. In a study by Mills et al. (2013), vascular responses in children measuring flow-mediated dilation (FMD) 
of the blood vessels were assessed during high and low intensity exergames (respectively $200 \mathrm{~m}$ hurdles and bowling). Only $15 \mathrm{~min}$ of playing a high intensity exergame produced significant changes in FMD. The intensity was moderate with a VO2max of $19.1 \pm 2.8 \mathrm{~mL} / \mathrm{kg} / \mathrm{min}$ (Mills et al., 2013). A 12-week dance exergame intervention produced significant improvement in FMD, overall exercise time, mean arterial pressure and VO2max compared to non-exercising counterparts $(2.38 \pm 3.91$ compared with $1.23 \pm 3.18 \mathrm{mg} / \mathrm{kg} / \mathrm{min} ; p=0.005)$. Even though the available data cannot clarify the underlying psychological mechanisms, numerous studies have shown that PA interventions for children and adolescents can enhance physical but also mental health including self-confidence, selfefficacy, and an improved mindset of mental toughness (Brand et al., 2016; Gerber et al., 2013; Hassmen, Koivula, \& Uutela, 2000; Liu, Wu, \& Ming, 2015).

The strength of the present study is that it seems to be the first to investigating the effects of a long-term AET intervention in a paediatric group of IBD-patients. Further, it employed an innovative intervention method with satisfactory adherence rates (77-89\%), as well as a broad range of internationally validated subjective and objective instruments to assess psychological functioning, sleep and PA. The empirical evidence generated by our exploratory trial can be used to formulate hypothesis in future, large-scale studies. Specifically, the effect sizes provided in this exploratory trial will help to estimate the minimal sample size in forthcoming, randomized controlled trials.

However, various limitations warrant against over generalization, and the findings should be interpreted cautiously. First, the sample size of our exploratory trial was small. Second, recruitment of the sample was restricted to the German speaking part of Switzerland and only participants willing and able to participate were assessed. Third, no non-intervention control group was included; this limited the potential of the study to detect intervention effects. Fourth, the children and adolescents with IBD were in rather good health and by definition not inactive at baseline, leaving only limited space for significant improvement. Fifth, the intervention period of eight weeks may have been too short to induce relevant changes, while the intensity of the intervention, and the frequency and duration of the single bouts of the intervention might have been suboptimal. Sixth, symptoms of depression were exclusively assessed via self-report. Seventh, even though we tried to cover an extensive range of factors, it remains conceivable that the pattern of results emerged due to further latent, but unassessed psychological or physiological variables. Eighth, given the nature and structure of the intervention, patients were not unaware of the study condition and therefore we cannot rule out the possibility that treatment effects were due to participants' expectations and motivations. In this context, one suggestion for future studies is to design randomized controlled trials with non-intervention control groups, using 


\section{Chapter 5}

larger samples, extending the intervention period and investigating the effectiveness of different exercise intensities.

\section{Conclusion}

The intervention of an 8-week exergaming intervention in paediatric patients with IBD and healthy controls had specific favorable impacts on objective sleep, exercise capacity, self-reported fitness and daily physical activity, while neither positive nor negative changes were observed for psychological functioning. Thus, regular physical activity seems to have no adverse impact on paediatric patients with IBD.

\section{Funding}

This work was supported by Freiwillige Akademische Gesellschaft Basel (FAG, Basel, Switzerland).

\section{Author contributions}

Conception and design of the study, acquisition of data, analysis and interpretation of data: LM, MG, RF, CL, NK, EHT, SB; drafting the article or revising it critically for important intellectual content: LM, MG, RF, CL, NK, EHT, SB; final approval of the version submitted: LM, MG, RF, CL, NK, EHT, SB. The manuscript is not under consideration for publication elsewhere.

\section{Declaration of conflicting interests}

The authors declare that there is no conflict of interest.

\section{Acknowledgements}

We thank the Kantonsspital Aarau for providing additional recruitment options, as well as Marielle Koenig and Vladimir Djurdjevic for sleep EEG scoring. Moreover, we are grateful to Noe Stoll for support during data collection and data entry. Finally, we thank Nick Emler (University of Surrey, UK) for proofreading the manuscript. This work was supported by Freiwillige Akademische Gesellschaft Basel (FAG, Basel, Switzerland). 


\section{References}

1 Bandura, A. (1977). Self-efficacy: Toward a unifying theory of behaviour change. Psychological Reviews, 84(2), 191e215.

2 Bastien, C. H., Vallieres, A., \& Morin, C. M. (2001). Validation of the Insomnia Severity Index as an outcome measure for insomnia research. Sleep Medicine, 2(4), 297-307.

3 Bilski, J., Mazur-Bialy, A. I., Wierdak, M., \& Brzozowski, T. (2013). The impact of physical activity and nutrition on inflammatory bowel disease: The potential role of cross talk between adipose tissue and skeletal muscle. Journal of Physiology Pharmacology, 64(2), 143-155.

4 Bishop, J., Lemberg, D. A., \& Day, A. (2014). Managing inflammatory bowel disease in adolescent patients. Adolescent Health, Medicine and Therapeutics, 5, 1-13.

5 Booth, F. W., Roberts, C. K., \& Laye, M. J. (2012). Lack of exercise is a major cause of chronic diseases. Comprehensive Physiology, 2(2), 1143-1211.

6 Borg, G. (1998). Borg's perceived exertion and pain scales. Champaign IL: Human Kinetics.

7 Bosco, F. A., Aguinis, H., Singh, K., Field, J. G., \& Pierce, C. A. (2015). Correlational effect size benchmarks. Journal of Applied Psychology, 100(2), 431-449.

8 Brand, S., Kalak, N., Gerber, M., Clough, P. J., Lemola, S., Sadeghi Bahmani, D., et al. (2016). During early to mid adolescence, moderate to vigorous physical activity is associated with restoring sleep, psychological functioning, mental toughness and male gender. Journal of Sports Science, 1-9.

9 Brand, S., \& Kirov, R. (2011). Sleep and its importance in adolescence and in common adolescent somatic and psychiatric conditions. International Journal of General Medicine, 4, 425-442.

10 Chennaoui, M., Arnal, P. J., Sauvet, F., \& Leger, D. (2015). Sleep and exercise: A reciprocal issue? Sleep Medicine Reviews, 20, 59-72.

11 Chouliaras, G., Margoni, D., Dimakou, K., Fessatou, S., Panayiotou, I., \& Roma- Giannikou, E. (2017). Disease impact on the quality of life of children with inflammatory bowel disease. World Journal of Gastroenterology, 23(6), 1067-1075.

12 Craig, C. L., Marshall, A. L., Sjostrom, M., Bauman, A. E., Booth, M. L., Ainsworth, B. E., et al. (2003). International physical activity questionnaire: 12 -country reliability and validity. Medicine and Science in Sports and Exercise, 35, 1381-1395.

13 De Boer, M., Grootenhuis, M., Derkx, B., \& Last, B. (2005). Health-related quality of life and psychosocial functioning of adolescents with inflammatory bowel disease. Inflammatory Bowel Diseases, 11(4), 400-406.

14 D'Inc`a, R., Varnier, M., Mestriner, C., Martines, D., D'Odorico, A., \& Sturniolo, G. C. (1999). Effect of moderate exercise on Crohn's disease patients in remission. Italian Journal of Gastroenterology and Hepatology, 31(3), 205-210.

15 Elsenbruch, S., Langhorst, J., Popkirowa, K., Müller, T., Luedtke, R., Franken, U., et al. (2005). Effects of mind-body therapy on quality of life and neuroendocrine and cellular immune functions in patients with ulcerative colitis. Psychotherapy and Psychosomatics, 74(5), 277-287.

16 Fruhe, B., Allgaier, A. K., Pietsch, K., Baethmann, M., Peters, J., Kellnar, S., et al. (2012). Children's depression screener (ChilD-S): Development and validation of a depression screening instrument for children in pediatric care. Child Psychiatry and Human Development, 43(1), 137151.

17 Gerbarg, P. L., Jacob, V. E., Stevens, L., Bosworth, B. P., Chabouni, F., DeFilippis, E. M., et al. (2015). The effect of breathing, movement, and meditation on psychological and physical symptoms and inflammatory biomarkers in inflammatory bowel disease: A randomized controlled trial. Inflammatory Bowel Diseases, 21(12), 2886-2896.

18 Gerber, M., Kalak, N., Lemola, S., Clough, P. J., Perry, J. L., Pühse, U., et al. (2013). Are adolescents with high mental toughness levels more resilient against stress? Stress and Health, 29(2), 164-171. 


\section{Chapter 5}

19 Gerber, M., Lang, C., Lemola, S., Colledge, F., Kalak, N., Holsboer-Trachsler, E., et al. (2016). Validation of the German version of the insomnia severity index in adolescents, young adults and adult workers: Results from three cross-sectional studies. BMC Psychiatry, 16, 174.

20 Greenley, R. N., Hommel, K. A., Nebel, J., Raboin, T., Li, S. H., Simpson, P., et al. (2010). A meta-analytic review of the psychosocial adjustment of youth with inflammatory bowel disease. Journal of Pediatric Psychology, 35(8), 857-869.

21 Gupta, N., Khera, S., Vempati, R. P., Sharma, R., \& Bijlani, R. L. (2006). Effect of yoga based lifestyle intervention on state and trait anxiety. Indian Journal of Physiology and Pharmacology, $50,41-47$.

22 Hagstromer, M., Bergman, P., De Bourdeaudhuij, I., Ortega, F. B., Ruiz, J. R., Manios, Y., et al. (2008). Concurrent validity of a modified version of the international physical activity questionnaire (IPAQ-A) in European adolescents: The HELENA study. International Journal of Obesity, 32(5), 42-48.

23 Hassan, J., van der Net, J., Helders, P. J., Prakken, B. J., \& Takken, T. (2010). Six-minute walk test in children with chronic conditions. British Journal of Sports Medicine, 44(4), 270-274.

24 Hassmen, P., Koivula, N., \& Uutela, A. (2000). Physical exercise and psychological well-being: A population study in Finland. Preventive Medicine, 30(1), 17-25.

25 IPAQ. (2005). Guidelines for data processing and analysis of the international physical activity questionnaire (IPAQ) e short and long forms, revised on November 2005. Retrieved March 15, 2010, from http://www.ipaq.ki.se/scoring.pdf.

26 Josefsson, T., Lindwall, M., \& Archer, T. (2014). Physical exercise intervention in depressive disorders: meta-analysis and systematic review. Scandinavian Journal of Medicine \& Science in Sports, 24(2), 259-272.

27 Jossen, J., \& Dubinsky, M. (2016). Therapeutic drug monitoring in inflammatory bowel disease. Current Opinion in Pediatrics, 28(5), 620-625.

28 Kalak, N., Gerber, M., Kirov, R., Mikoteit, T., Yordanova, J., Puhse, U., et al. (2012). Daily morning running for 3 weeks improved sleep and psychological functioning in healthy adolescents compared with controls. Journal of Adolescent Health, 51(6), 615-622.

29 Keerthy, D., Youk, A., Srinath, A. I., Malas, N., Bujoreanu, S., Bousvaros, A., et al. (2016). Effect of psychotherapy on health care utilization in children with inflammatory bowel disease and depression. Journal of Pediatric Gastroenterology and Nutrition, 63(6), 658-664.

30 Kinnucan, J. A., Rubin, D. T., \& Ali, T. (2013). Sleep and inflammatory bowel disease: Exploring the relationship between sleep disturbances and inflammation. Gastroenterology \& Hepatology (N Y), 9(11), 718-727.

31 Klare, P., Nigg, J., Nold, J., Haller, B., Krug, A. B., Mair, S., et al. (2015). The impact of a tenweek physical exercise program on health-related quality of life in patients with inflammatory bowel disease: A prospective randomized controlled trial. Digestion, 91(3), 239-247.

32 Lang, C., Brand, S., Feldmeth, A. K., Holsboer-Trachsler, E., Puhse, U., \& Gerber, M. (2013). Increased self-reported and objectively assessed physical activity predict sleep quality among adolescents. Physiology \& Behaviour, 120, 46-53.

33 Lang, C., Kalak, N., Brand, S., Holsboer-Trachsler, E., Puhse, U., \& Gerber, M. (2016). The relationship between physical activity and sleep from mid adolescence to early adulthood. A systematic review of methodological approaches and meta- analysis. Sleep Medicine Reviews, 28 , $32-45$.

34 Levine, A., de Bie, C. I., Turner, D., Cucchiara, S., Sladek, M., et al. (2013). Atypical disease phenotypes in pediatric ulcerative colitis: 5-year analyses of the EUROKIDS registry. Inflammatory Bowel Diseases, 19(2), 370-377.

35 Liu, M., Wu, L., \& Ming, Q. (2015). How does physical activity intervention improve self-esteem and self-concept in children and Adolescents? Evidence from a meta-analysis. PLoS One, 10(8), e0134804. 
36 Loudon, C. P., Corroll, V., Butcher, J., Rawsthorne, P., \& Bernstein, C. N. (1999). The effects of physical exercise on patients with Crohn's disease. The American Journal of Gastroenterology, 94(3), 697-703.

37 Mackner, L. M., \& Crandall, W. V. (2005). Long-term psychosocial outcomes reported by children and adolescents with inflammatory bowel disease. The American Journal of Gastroenterolology, 100(6), 1386-1392.

38 Maddison, R., Mhurchu, C. N., Jull, A., Prapavessis, H., Foley, L. S., \& Jiang, Y. (2012). Active video games: The mediating effect of aerobic fitness on body composition. International Journal of Behavioral Nutrition and Physical Activity, 9, 54.

39 Meltzer, L. J., Hiruma, L. S., Avis, K., Montgomery-Downs, H., \& Valentin, J. (2015). Comparison of a commercial accelerometer with polysomnography and actigraphy in children and adolescents. Sleep, 38(8), 1323-1330.

40 Mikocka-Walus, A., Knowles, S. R., Keefer, L., \& Graff, L. (2016). Controversies revisited: A systematic review of the comorbidity of depression and anxiety with inflammatory bowel diseases. Inflammatory Bowel Diseases, 22(3), 752-762.

41 Mills, A., Rosenberg, M., Stratton, G., Carter, H. H., Spence, A. L., Pugh, C. J., et al. (2013). The effect of exergaming on vascular function in children. Journal of Pediatrics, 163(3), 806-810.

42 Narula, N., \& Fedorak, R. N. (2008). Exercise and inflammatory bowel disease. Canadian Journal of Gastroenterology, 22(5), 497-504.

43 Ng, S. C., Tang, W., Leong, R. W., Chen, M., Ko, Y., Studd, C., et al. (2015). Environ- mental risk factors in inflammatory bowel disease: A population-based case- control study in asia-pacific. Gut, 64(7), 1063e1071.

44 Nicholas, D. B., Otley, A., Smith, C., Avolio, J., Munk, M., \& Griffiths, A. M. (2007). Challenges and strategies of children and adolescents with inflammatory bowel disease: A qualitative examination. Health and Quality of Life Outcomes, 5, 28.

45 Ottevaere, C., Huybrechts, I., De Bourdeaudhuij, I., Sjostrom, M., Ruiz, J. R., Ortega, F. B., et al. (2011). Comparison of the IPAQ-a and actigraph in relation to VO2max among European adolescents: The HELENA study. Journal of Science and Medicine in Sport, 14(4), 317-324.

46 Packer, N., Hoffman-Goetz, L., \& Ward, G. (2010). Does physical activity affect quality of life, disease symptoms and immune measures in patients with inflammatory bowel disease? A systematic review. Journal of Sports Medicine and Physical Fitness, 50(1), 1-18.

47 Peng, W., Lin, J. H., \& Crouse, J. (2011). Is playing exergames really exercising? A meta-analysis of energy expenditure in active video games. Cyberpsychology, Behavior and Social Networking, 14(11), 681-688.

48 Ravens-Sieberer, U., Gosch, A., Rajmil, L., Erhart, M., Bruil, J., Power, M., et al. (2008). The KIDSCREEN-52 quality of life measure for children and adolescents: Psychometric results from a cross-cultural survey in 13 European countries. Value Health, 11(4), 645e658.

49 Rechtschaffen, A., \& Kales, A. (1968). A manual of standardized terminology, tech- niques and scoring system for sleep stages of human subjects (Vol. 204). NIH Publ.

50 Russel, M. G. (2000). Changes in the incidence of inflammatory bowel disease: What does it mean? European Journal of Internal Medicine, 11(4), 191-196.

51 Schoech, D., Boyas, J. F., Black, B. M., \& Elias-Lambert, N. (2013). Gamification for behavior Change: Lessons from developing a social, multiuser, web-tablet based prevention game for youths. Journal of Technology in Human Services, 31(3), 197-217.

52 Scott, F. I., \& Lichtenstein, G. R. (2016). Advances in therapeutic drug monitoring of biologic therapies in inflammatory bowel disease: 2015 in review. Current Treatment Options in Gastroenterology, 14(1), 91-102.

53 Stanton, R., \& Reaburn, P. (2014). Exercise and the treatment of depression: A review of the exercise program variables. Journal of Science and Medicine in Sport, 17(2), 177-182.

54 Sween, J., Wallington, S. F., Sheppard, V., Taylor, T., Llanos, A. A., \& Adams- Campbell, L. L. (2014). The role of exergaming in improving physical activity: A review. Journal of Physical Activity \& Health, 11(4), 864-870. 


\section{Chapter 5}

55 Tanaka, H., Monahan, K. D., \& Seals, D. R. (2001). Age-predicted maximal heart rate revisited. Journal of the American College of Cardiology, 37(1), 153-156.

56 Timmer, A., Preiss, J. C., Motschall, E., Rucker, G., Jantschek, G., \& Moser, G. (2011). Psychological interventions for treatment of inflammatory bowel disease. Cochrane Database of Systematic Reviews, 2, Cd006913.

57 Turner, D., Griffiths, A. M., Walters, T. D., Seah, T., Markowitz, J., Pfefferkorn, M., et al. (2010). Appraisal of the pediatric Crohn's disease activity index on four prospectively collected datasets: Recommended cutoff values and clinimetric properties. American Journal of Gastroenterology, 105(9), 2085-2092.

58 Ulrich, S., Hildenbrand, F. F., Treder, U., Fischler, M., Keusch, S., Speich, R., et al. (2013). Reference values for the 6-minute walk test in healthy children and adolescents in Switzerland. BMC Pulmonary Medicine, 13, 49.

59 Vaisto, T., Aronen, E. T., Simola, P., Ashorn, M., \& Kolho, K. L. (2010). Psychosocial symptoms and competence among adolescents with inflammatory bowel disease and their peers. Inflammatory Bowel Diseases, 16(1), 27-35.

60 Wanrooij, V. H., Willeboordse, M., Dompeling, E., \& van de Kant, K. D. (2014). Exercise training in children with asthma: A systematic review. British Journal of Sports Medicine, 48(13), 10241031.

61 Werkstetter, K. J., Ullrich, J., Schatz, S. B., Prell, C., Koletzko, B., \& Koletzko, S. (2012). Lean body mass, physical activity and quality of life in paediatric patients with inflammatory bowel disease and in healthy controls. Journal of Crohns and Colitis, 6(6), 665-673. 


\section{Chapter 6}

Favorable impact of acute and chronic aerobic exercise training on disease symptoms and cardiovascular fitness in children and adolescents with inflammatory bowel disease

Drafted as: MähImann L, Gerber M, Legeret C, Köhler H, Kalak N, HolsboerTrachsler E, Brand S, Furlano R: Favorable impact of chronic aerobic exercise training on disease symptoms in children and adolescents with inflammatory bowel disease. In preparation for submission 2018. 


\section{Chapter 6}

\section{Abstract}

Background: Standard treatment for children and adolescents with IBD are immune modulating pharmaceuticals, however, evidence is growing that acute and chronic exercise has the potential to impact on the physiological system related to cardiovascular fitness and inflammatory indices.

Method: 21 children and adolescents with IBD were compared to 23 gender- and age-matched healthy controls $(\mathrm{HC})$. The IBD group was split into a "remissiongroup" (IBD-RE; $n=14$ ) and an "active disease group" (IBD-AD; $n=7)$. All participants completed a single exercise bout at baseline and study end (6MWT) and an 8-week exercise intervention reaching 60\% VO2max for 5 days per week. Before and after each short exercise bout, IBD-related inflammatory indices (erythrocyte sedimentation rate (ESR), albumin, C-reactive protein (CRP), cortisol, hemoglobin, hematocrit, thrombocytes and leukocytes) were assessed. Further, at baseline and after 8 weeks, objective measures of steps per day for 5 consecutive days, exercise capacity and HR were assessed.

Results: After a single exercise bout (6MWT) albumin, haemoglobin, erythrocytes, haematocrit and leukocytes increased in all three groups IBD-AD, IBD-RE and HC. CRP and thrombocytes were elevated in IBD-AD and IBD-RE as compared to $\mathrm{HC}$. Concerning group $x$ time interactions increased erythrocytes were reported in IBD-AD and IBD-RE, while they decreased among HC. After a longterm AET intervention, ESR, CRP and thrombocytes significantly decreased in all groups. Comparing groups, ESR was highest in IBD-AD, followed by IBD-RE and $\mathrm{HC}$. Concerning group $x$ time interactions, decreased values were observed over time in IBD-AD and IBD-RE, while values for HC increased.

Conclusion: While acute moderate PA enhances inflammatory markers, longterm moderate PA reduced inflammatory markers, exercise capacity and physical activity in children and adolescents with IBD. The pattern of results suggests that moderate regular PA may be recommended as an adjuvant anti-inflammatory therapy in paediatric IBD. 


\section{Introduction}

Inflammatory bowel disease (IBD) is a chronic, relapsing and remitting autoimmune disease that manifests in two major forms of chronic intestinal inflammation: Crohn's disease (CD) and ulcerative colitis (UC), as well as atypical phenotypes. ${ }^{1}$ Underlying causes of the dysregulated inflammation are still unclear. Current theories acknowledge interactions between the intestinal microbiome and the mucosa, especially in genetically predisposed hosts ${ }^{2}$, as well as microbial and environmental factors. ${ }^{3-5}$ The chronic inflammatory state is accompanied by a high production of pro-inflammatory cytokines and causes clinical symptoms like colon ulcers, rectal bleeding, diarrhea, abdominal pain, fatigue, an overall altered physical and emotional well-being ${ }^{9}$ and leads towards an increased risk of colorectal cancer later in life. ${ }^{10}$ The current standard of care for paediatric IBD patients includes a combination of pharmacotherapy, surgical intervention, and/or enteral nutrition, aiming to minimize or reduce inflammation, relieve symptoms, improve quality of life and eschew long-term complications. ${ }^{15}$ Specifically, medical surveillance of mucosal inflammation in the gut typically coincides with the prescription of anti-inflammatory and immunosuppressant pharmaceuticals, even though their efficacy is incomplete and their side effects severe. ${ }^{16,17}$

Conversely, physical activity (PA), defined as any body movement that will result in energy expenditure, has frequently been suggested as adjunct therapy option prevention and prognosis of cancer, cardiovascular diseases, diabetes and chronic inflammatory diseases. ${ }^{9}$ Next to the well-established favourable effects on physical, psychological/social and cognitive effects ${ }^{15}$, PA has been documented to have immune modulating potential. ${ }^{18}$ Even though we still lack a thorough understanding of the underlying immunologic mechanisms several theories have been discussed in detail in Sharif (2018). ${ }^{18}$ Summarized, the anti-inflammatory effect of PA results from a decrease in visceral fat, significant elevation of Tregulatory cells, decreased immunoglobulin secretion and release of myokines including interleukine 6 (IL-6) from muscles. In the case of IBD, regular physical activity has been reported to improve exercise capacity ${ }^{19}$, boost mental health and stress management ${ }^{20,21}$, exert anabolic properties that stimulate bone growth and muscle strength ${ }^{22}$, may alter the body composition and inhibit the development of mesenteric white adipose tissue ${ }^{23}$, may influence the diversity of the microbiome ${ }^{24}$, may counteract carcinogenesis ${ }^{25}$ and is again considered anti-inflammatory. ${ }^{18}$ However, patients with IBD still seem to be reluctant and have been reported as less active as compared to healthy controls, potentially due to their fear of symptom exerbation and health professionals.

According to the current state of evidence, in detail, for acute physical activity, D'Inca et al. ${ }^{27}$ found that after a short bout of one hour $60 \%$ Vo2max cycle ergometry, subjects did not report elicit symptoms or chances in intestinal 
permeability and lipid peroxidation. Further, Ploeger et al. ${ }^{19}$ tested children and adolescents during 30 min of cycling on either $50 \%$ of peak mechanical power or 6 bouts of $4 \times 15 \mathrm{sec}$ of cycling at $100 \%$ peak mechanical power. Results indicated no significant exacerbation of the disease or inflammatory cytokines responses in either of the two exercises. Furthermore, both types of exercise spontaneously increased the numbers of immune cells, and growth hormone $(\mathrm{GH})$ concentrations and decreased levels of IGF-1, which indicated an immune reaction. ${ }^{28}$

Concerning the influence of long-term physical activity interventions on patients with IBD, so far four systematic reviews have been published by Narula \& Fedorak ${ }^{29}$; Packer, Hoffman-Goetz, \& Ward ${ }^{30}$ and Shepherd. ${ }^{28}$ Based on these reviews, nine unique intervention studies were identified. ${ }^{27,31-38}$ Different exercise interventions were applied including moderate cycling ${ }^{27}$, low-intensity walking $^{33,35}$, yoga ${ }^{32}$, stress management training, moderate exercise, Mediterranean diet, behavioral techniques and self-care strategies ${ }^{31}$, 10-week low-intensity physical activity-program (three times per week) 34 ; two individual person-centered exercise consultations during 12 weeks ${ }^{36}$, individual advice regarding physical activity during 12 weeks by a physiotherapist ${ }^{37}$, and home-base low impact exercise program twice a week for 12 months. ${ }^{38}$ None of studies reported exuberated bowel symptoms, but improvement in the health status due to improved immune and physical functioning. In detail, the study by Robinson et al. ${ }^{38}$ among adults found increased bone mineral density in lumbar spine and hips after low impact exercise program; Louden et al. ${ }^{33}$, Elsenbruch et al. ${ }^{31}$ and $\mathrm{Ng}$ et al. ${ }^{39}$ found that low-intensity walking or light exercise improved physical health, general wellbeing, diminished stress and improved quality of life and reduction in CD symptoms.

To summarize, even though, first studies indicate a beneficial effect of physical activity for pediatric IBD, the effectiveness of PA on the multifactorial disease impact of IBD has not been well described. Based on this previous knowledge we designed a larger multidimensional project investigating the importance of PA for pediatric IBD. Summarized, the three previous articles all based on the one cohort, showed the following: In the baseline comparison, participants with IBD in an active state of the disease (IBD-AD) showed higher markers of inflammation including erythrocyte sedimentation rate (ESR), C-reactive protein (CRP) values, haemoglobin, and leukocyte values. IBD-AD had poorer psychological functioning and lower PA (average steps per day) compared to participants with IBD in remission (IBD-RE) and healthy controls (HC). In the second article, sleep behavior was investigated in more detail. Compared to HC and IBD-RE, IBD-AD patients showed impaired objective sleep patterns (eg, more awakenings, longer sleep latency, and reduced stage 3 sleep). In the third article, an 8-week physical activity intervention improved exercise capacity of all participants. Self-reported fitness and daily physical activity increased in IBD-AD, but not in IBD-RE and HC. 
No improvements were observed for psychological functioning, depressive symptoms or subjective sleep over time. Objective sleep improved, deep sleep increased, light sleep decreased, and in the IBD-AD group the number of arousals after sleep onset decreased.

However, the remaining question is, whether acute or long-term PA has an impact on disease activity and favorable effects on the mechanics immunological adaptions. Currently, meachnics are poorly understood and limited to the adult population. ${ }^{22,30}$ As a result, non-pharmacological treatments remain scarce and current treatment standards fail to consider the potential benefits of increasing the typically low levels of daily physical activity. ${ }^{28}$

We took all previous observations into account. The aim of the present study was to investigate the influence of a short exercise bout and a long-term exercise intervention using an exergame approach on inflammatory indices (C-reactive Protein (CRP) erythrocyte sedimentation rate (ESR), Thrombocytosis) and exercise capacity among children and adolescents with IBD and healthy controls. We believe that the present findings may help paediatricians and specifically paediatricians of gastroenterology to improve treatment of peadiatric patients suffering from IBD by allowing adjuvant lifestyle treatment targets to be formulated.

The following three hypotheses were formulated: First, following further findings of Ploeger et al. ${ }^{19}$ and D'Inca et al. ${ }^{27}$, we expected higher inflammatory indices after an acute bout of exercise in children and adolescents with IBD. Second, following previous findings of the reviews by Narula and Fedorak ${ }^{29}$, Packer et al. ${ }^{30}$ and Shephard ${ }^{28}$, we expected that a long term AET intervention favorably impacts the inflammatory blood markers (thrombocyte count and ESR) in children and adolescents with IBD.

\section{Methods}

\section{Procedure}

As previously described elsewhere, in this case-control 8-week longitudinal AET interventional study participants were recruited from the University Children's Hospital Basel (Switzerland). The participants were invited to the hospital to perform a baseline assessment and a second assessment immediately after the AET intervention. All data were collected by two trained research assistants and supervised by a medical doctor. First, anthropometric measures height, weight, waist circumference (measured $4 \mathrm{~cm}$ above the navel using a standard anthropometry tape) compared to reference values established by Taylor et al. ${ }^{40}$ and BMI $\left(\mathrm{kg} / \mathrm{m}^{2}\right)$ which were compared to the WHO international growth references ${ }^{41}$, were assessed. Second, participants' blood values were collected. 


\section{Chapter 6}

The present study was approved by the local ethical committee (EKNZ: 2014:220). Furthermore, the study was conducted in accordance to the ethical principles laid down in Declaration of Helsinki (Trial registration number: NCT02264275).

\section{Sample}

Twenty-one children and adolescents with IBD (mean age: 13.88 years; female $\mathrm{n}=10[43.5 \%]$ ) were recruited at the University Children's Hospital Basel (UKBB, Basel Switzerland) and enrolled in the study during December 2014 and November 2015. A diagnosis of IBD was clinically confirmed and patients were subdivided according to the activity scores of their disease (PUCAI (Paediatric ulcerative colitis activity index) scores and Paediatric Crohn's disease activity index (clinical scores) ${ }^{42}$ : seven participants were in an active disease state (IBD-AD) and 14 in remission (IBD-RE) during the study period. In parallel, a healthy control group $(\mathrm{HC})$ matched for age and gender was recruited (mean age: 12.38 years, females $n=15$ [62.5\%]) through word-of-mouth-recommendation. All participants were fully informed about the aims of the present study, the voluntary and confidential basis of their participation. Thereafter, written and informed consent was signed both by participants and their legal guardians.

Table 1. Sample Characteristics

\begin{tabular}{|c|c|c|c|}
\hline & $\begin{array}{l}\text { IBD-AD } \\
(\mathrm{N}=7)\end{array}$ & $\begin{array}{l}\text { IBD-RE } \\
(N=14)\end{array}$ & $\begin{array}{l}\mathrm{HC} \\
(\mathrm{N}=23)\end{array}$ \\
\hline Age in months & $176.25 \pm 39.03$ & $158.79 \pm 35.53$ & $148.58 \pm 38.84$ \\
\hline Female & $4(50)$ & $6(42.9)$ & $15(62.5)$ \\
\hline \multicolumn{4}{|l|}{ IBD } \\
\hline Colitis Ulcerosa & $3(37.5)$ & $4(28.6)$ & \\
\hline Crohn's Diesease & $5(62.5)$ & $7(50)$ & \\
\hline Undefined Colitis & & $3(21.4)$ & \\
\hline Time since diagnosis & $111.54 \pm 83.35$ & $124.74 \pm 88.19$ & \\
\hline Waist circumference $(\mathrm{cm})$ & $67.63 \pm 9.04$ & $65.65 \pm 7.17$ & $64.48 \pm 8.29$ \\
\hline Difference from norm & $-9.81 \pm 6.15$ & $-8.68 \pm 4.95$ & $-8.82 \pm 6.0$ \\
\hline Height $(\mathrm{cm})$ & $153.48 \pm 17.53$ & $152.69 \pm 12.02$ & $152.26 \pm 18.0$ \\
\hline Weight to height (\%) & $44.08 \pm 3.07$ & $42.94 \pm 2.13$ & $42.77 \pm 3.49$ \\
\hline Weight (kg) & $45.28 \pm 15.85$ & $42.79 \pm 12.83$ & $44.37 \pm 15.75$ \\
\hline BMI & $18.6 \pm 3.37$ & $17.91 \pm 2.78$ & $18.36 \pm 3.16$ \\
\hline BMI z-score & $-0.588 \pm 0.79$ & $-0.34 \pm 0.95$ & $-0.19 \pm 0.87$ \\
\hline Percentile BMI vs Age & $31.98 \pm 24.31$ & $39.82 \pm 26.23$ & $43.89 \pm 26.43$ \\
\hline z-score Height vs Age & $-0.911 \pm 1.73$ & $0.02 \pm 0.86$ & $0.19 \pm 1.01$ \\
\hline Percentile Height vs Age & $32.04 \pm 32.99$ & $52.91 \pm 25.71$ & $54.83 \pm 31.53$ \\
\hline
\end{tabular}

* Notes: $\mathrm{N}=44$, IBD-AD = IBD in an active state of the disease, IBD-RE = IBD in remission, $\mathrm{HC}=$ Healthy Control; all data presented in mean \pm standard deviation 
The sample was subdivided into three groups according to the regularly assessed PUCAI (Paediatric ulcerative colitis activity index) scores and PCDAI (Paediatric Crohn's disease activity index) (clinical scores) ${ }^{42}$ : IBD with active disease (IBD-AD), IBD in remission (IBD-RE) and healthy control $(\mathrm{HC})$ are represented in Table 1.

\section{Tools}

\section{Laboratory assessment}

A blood sample using the finger prick technique was applied to measure inflammatory indices such as C-reactive protein (CRP) $[\mathrm{g} / \mathrm{dl}]$, thrombocyte $[\mathrm{g} / \mathrm{dl}]$ and leucocyte $[\mathrm{g} / \mathrm{dl}]$ count, as well as haemoglobin $(\mathrm{Hb})[\mathrm{g} / \mathrm{dl}]$, albumin, cortisol and erythrocyte sedimentation rate [ESR].

\section{Short bout PA Intervention}

The submaximal 6-minute walking test (6MWT) was applied as a short bout PA intervention. It is a self-paced, submaximal exercise test and measures the maximal distance a participant is able to walk over six minutes. ${ }^{48}$ The 6 MWT has been validated as suitable for paediatric populations with chronic diseases. ${ }^{49}$ Heart rate (HR) is continuously recorded during exercise using an elastic chest strap with heart rate sensor.

\section{Long term AET Intervention}

As described in elsewhere, the AET intervention incorporated an exergame or active video gameplay. Exergames were selected since they are easily accessible with adjustable levels of intensity to minimalize perceived barriers, perceived threads and as an intervention to facilitate action taking in a safe environment. Exergames are gaining popularity in as tools for physical activity interventions. Utilizing exergames as adjunct to daily physical activity has been chiefly supported in the literature in children and adolescents. ${ }^{51-58}$ Exergames can elevate energy expenditure to a moderate or vigorous intensity, metabolically equivalent to a three mile per hour treadmill pace..$^{51}$ Furthermore, gamification techniques and mechanisms enable to achieve goals, encourage players through rewards and points, while offering intrinsic motivation in the form of fun. ${ }^{59}$ Finally, selfefficacy ${ }^{60}$ was targeted as the feeling of confidence in one's own ability to achieve intended results. In the exergame context, this can be translated in the ability to earn points in the game, which provides children with the sense of accomplishment. Closely related, through the visualization of feedback and success after each single round of playing the exergame, as well as the overview of points for 


\section{Chapter 6}

longer time periods, increased motivation and intervention adherence is expected.

After the review of various exergames and their intensity levels, we decided to use the Just Dance Kids $₫$ and Sports-Resort $₫$ exergame for Nintendo Wii $\circledast$ in the present study. Just Dance Kids $®$ is a motion-based dancing game offering the player a collection of songs with accompanied dance choreography. During the songs, players mirror the dance performance and comments displayed on the screen, and are awarded for their accuracy. In addition, gold moves are integrated, which result in bonus points if mirrored correctly. The Nintendo Wii SportsResorts ${ }^{\circledR}$ game allows children to participate in a sports game in a virtual world. Players can choose between sward play, wakeboarding, frisbee, archery, basketball, table tennis, golf, bowling, canoeing and cycling. Players can either compete against the video game or can play against a second player.

Children and adolescents were offered to choose one of the games and were encouraged to use the exergames for 30 minutes, five days a week for a total of eight weeks, reaching a heart rate intensity of between 60 and $80 \%$ of maximum (based on the commonly used 220-age formula. ${ }^{61}$ Participant's parents were asked to monitor the intervention frequency in a paper diary to keep track of intervention adherence over the entire intervention period.

\section{Statistical analyses}

The initial statistical assessment comprised a summary of descriptive statistics including means, standard deviations and percentages. The three groups were compared using one-way repeated measures analyses of variance (ANOVAs) with the diagnostic clusters (IBD-AD, IBD-RE and HC) and time (baseline vs. post-intervention) as fixed factors and anthropometric measures, blood values and physical activity as outcomes. Post-hoc tests after Bonferroni-Holm corrections for $p$-values were performed to examine differences between the three specific groups. The nominal level of significance was set at alpha $<.05$. Due to deviation of sphericity, Greenhouse Geisser corrected degrees of freedom and epsilon $\varepsilon$ value were used. Further, effect sizes for ANOVAs were reported as partial eta-squared $\left.\left[\eta_{p}{ }^{2}\right]\right)$ and considered as follows: small $(S)=.01>\eta_{p}{ }^{2}<.059$, medium $(\mathrm{M})=.06>\eta_{p}<.139$, or large $(\mathrm{L})=\eta_{p} \geq .14 .{ }^{62}$ All statistics were performed with SPSS $₫ 22.0$ (IBM Corporation, Armonk NY, USA) for Windows ${ }^{\circledR}$. 


\section{Results}

\section{Long term intervention fidelity}

Based on the activity diaries, adherence of the intervention playing 5 times per week for 8 consecutive weeks was accomplished by IBD-AD with $77.5 \%$, IBD-RE with $84.5 \%$ and $\mathrm{HC}$ with $88.7 \%$. Further, parents and children reported that the Nintendo Wii was an attractive and innovative exergaming tool.

\section{Effect of single exercise bout on inflammation marker}

Table 3 provides an overview of the descriptive and inferential statistics for blood markers before and after a single exercise bout (6 MWT). All results are presented separately by time (before vs after intervention), groups (IBD-AD vs. IBD$\mathrm{RE}$ vs. $\mathrm{HC}$ ), and interaction (group $x$ time).

Regarding inflammatory blood markers, over time albumin $(p=.00 ; E S=.3)$, haemoglobin ( $p=.01 ; E S=.16)$, erythrocytes $(p=.00 ; E S=.22)$, haematocrit $(p=.00$; $E S=.3)$ and leukocytes $(p=.00 ; E S=.49)$ increased in all three groups IBD-AD, IBD-RE and HC. No significant time effects have been reported for ESR, CRP, cortisol and thrombocytes.

Concerning group effects, CRP-values were significantly higher in IBD-AD and IBD-RE as compared to $\mathrm{HC}(p=.03 ; E S=.18)$. Furthermore, thrombocytes were highest in IBD-AD, followed by IBD-RE and lowest in HC ( $p=.00$; $E S=.29)$. No significant group effects have been reported for BSG, albumin, cortisol, haemoglobin, erythrocytes, haematocrit and leukocytes. Investigating effect sizes for group effects, a medium effect size indicated increased values for cortisol $(E S=.06)$ and leukocytes for IBD-AD and IBD-RE as compared to HC $(E S=.1)$. 


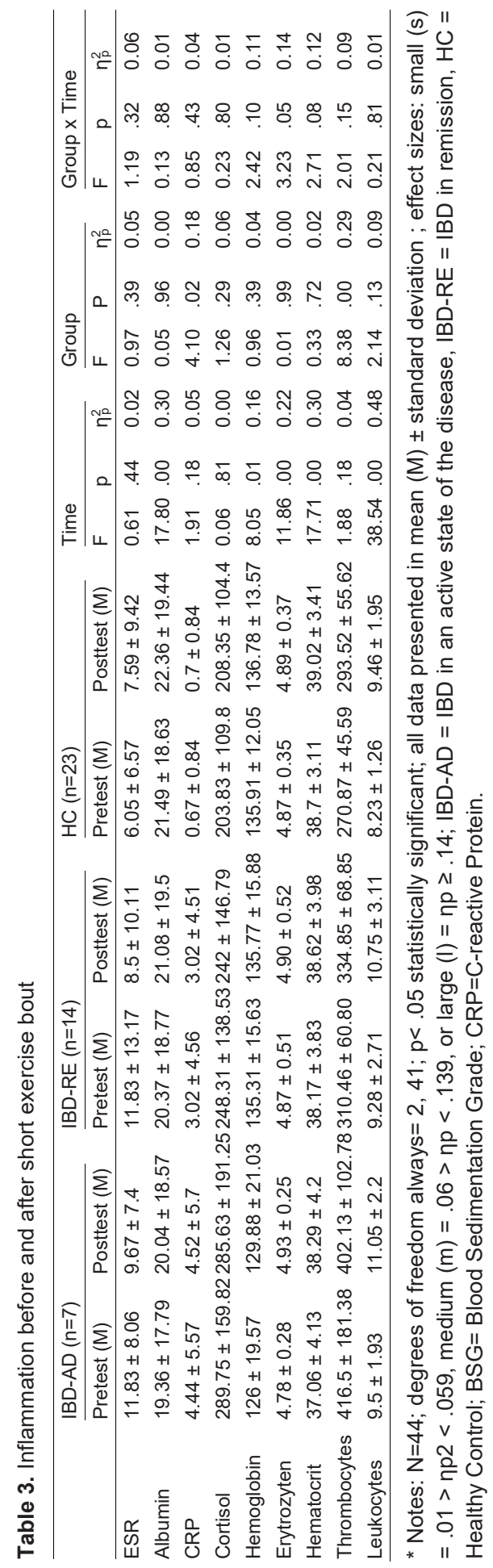




\section{Effect of longterm AET intervention on inflammatory marker}

Table 3 provides an overview of the descriptive and inferential statistics for blood markers before and after an 8-week AET intervention.

Over time, ESR values ( $p=.01 ; E S=.19)$ (fig.1), CRP ( $p=.00 ; E S=.23$ ) (fig 2) and thrombocytes $(p=.02 ; E S=.13)$ significantly decreased over time. Moreover, no significant time effects have been reported for albumin, cortisol, haemoglobin, erythrocytes, haematocrit and leukocytes. Investigating effect sizes, a medium effect size indicated increases for albumin ( $E S=.06)$.

Comparing groups, no significant group effects have been reported for ESR, albumin, CRP, cortisol, haemoglobin, erythrocytes, haematocrit, thrombocytes and leukocytes. Investigating effect sizes, a large effect size indicated strong decreases in ESR among IBD-AD and IBD-RE as compared to HC (ES=.14), a medium effect size indicated strong decreases in CRP among IBD-AD and IBD-RE as compared to $\mathrm{HC}(E S=.12)$ and a medium effect size indicated strong decreases in thrombocytes among IBD-AD and IBD-RE as compared to HC $(E S=.1)$.

Concerning interactions (time $\mathrm{x}$ group), significant results were observed for $\operatorname{ESR}(p=.02 ; E S=.19), \operatorname{CRP}(p=.02 ; E S=.20)$ and thrombocytes $(p=.00 ; E S=.3)$. Post hoc analysis with Bonferoni-Holm corrections for $p$ values revealed decreased values over time in IBD-AD and IBD-RE, while values for HC increased. Moreover, no significant time effects have been reported for ESR, albumin, cortisol, haemoglobin, erythrocytes, haematocrit and leukocytes. Investigating effect sizes, a medium effect size indicated an increase in haemoglobin among IBD$A D$, while IBD-RE stayed stable and $H C$ decreased $(E S=.08)$ and a medium effect size in haematocrit values showing strongest increase in IBD-AD, slight increase in IBD-RE and a decrease in $\mathrm{HC}(E S=.06)$. 


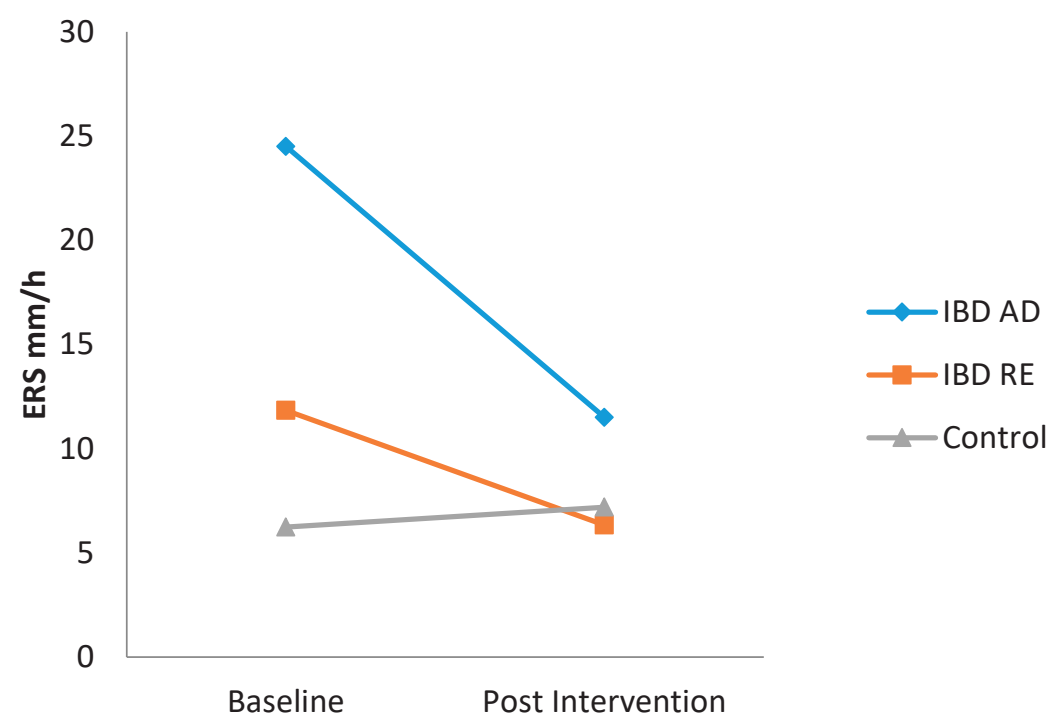

Figure 1. ESR decreased significantly over time $(p=.01)$ in all three groups. Points are means. IBD$A D$ : IBD in an active state of the disease; IBD-RE: IBD in remission; HC: Healthy Control.

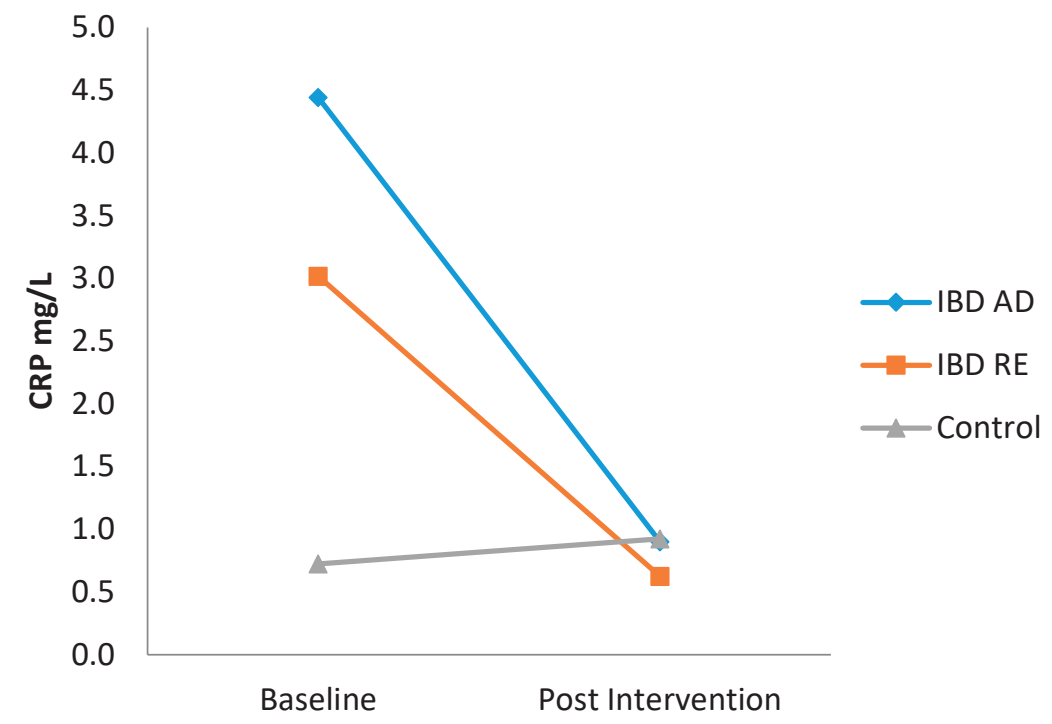

Figure 2. CRP decreased significantly over time $(p=.00)$ in all three groups. Points are means. IBD$A D$ : IBD in an active state of the disease; IBD-RE: IBD in remission; HC: Healthy Control. 


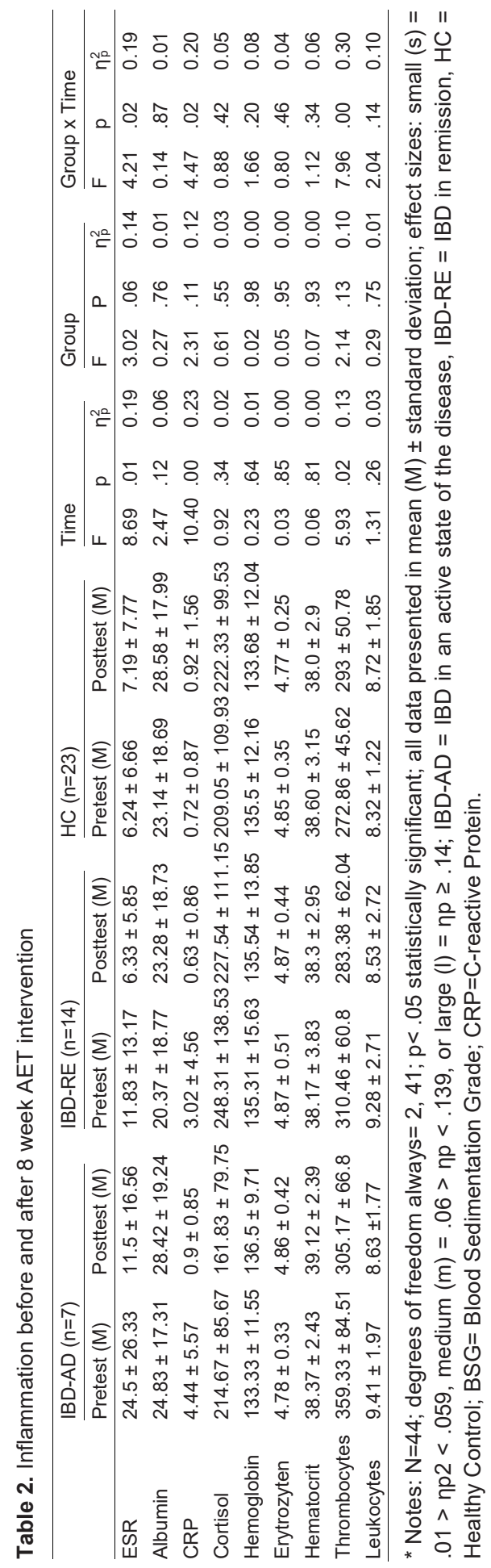




\section{Discussion}

The key findings of the present study were as followed: first, after a short bout of exercise, albumin, haemoglobin, erythrocytes, haematocrit and leukocytes significantly increased in all groups. And second, after the long-term intervention involving moderate PA during 5 weekdays for eight consecutive weeks, significantly reduced IBD specific acute and chronic inflammation markers CRP and $E S R$, as well as thrombocytes in IBD-AD. The results add to the current literature in showing that in a sample of children and adolescents with IBD-AD and IBD$\mathrm{RE}$, compared to healthy controls, first, acute exercise increased spontaneous inflammation irrespective of the initial inflammatory state, and, third, in contrast, light to moderate regular PA over a longer period does have a favourable impact on inflammation and physical functioning of children and adolescents with IBD.

The following three hypotheses were formulated and are considered now in turn:

First, following previous findings ${ }^{19,27}$, we expected that a short term exercise bout would spontaneously increase inflammatory blood markers (leukocyte, thrombocyte count and CRP) in children and adolescents with IBD. This hypothesis was confirmed by our findings. In IBD-AD and IBD-RE, CRP and thrombocytes were elevated after the single exercise bout, as compared to HC. Furthermore, leukocytes, albumin, haemoglobin, erythrocytes and haematocrit were elevated in all three groups after a single exercise bout (6 MWT). Findings of previous studies are discordant. Some previous studies reported no significant exacerbation of subjective or objective disease symptoms, intestinal permeability, lipid peroxidation or inflammatory cytokine response. ${ }^{19,27}$ However, a study by Ploeger et al. ${ }^{19}$ confirmed increasing cytokine levels after $4 \times 15$ s bouts of maximal cycle ergometry. In line, multiple studies among other chronic diseases confirmed the increased levels of circulating blood markers after a short bout of moderate to vigorous activity. ${ }^{65,66}$

The increase in circulating blood markers as immediate response to exercise could be caused by decreased plasma volume with an accompanied concentration effect ${ }^{66}$ and an activation of myocytes and macrophagues with subsequent upregulation and expression of proinflammatory cytokine, such as CRP. 67 For the observed leukocytosis, the following mechanism might be at play: Due to increases in cardiac output and blood flow, leukocytes from the vascular, pulmonary, hepatic, and/or splenic reservoirs are released increasingly. Equally important are catecholamines and glucocorticoids that bind and activate exercise responsive leukocytes during and after exercise. Moreover, exercise increases HPA axis activity and causes increased releases of stress hormones (corticotrophin releasing hormone, adrenocorticotrophic hormone and cortisol) which again have an effect on leukocyte trafficking. ${ }^{68}$ Hemoglobin is activated in its function 
as oxygen carrying pigment of the red blood cells from lungs to all tissue. ${ }^{69}$ The increased serum albumin levels indicate an increased need to maintain the bodies' fluid balance and supply proteins needed for growth and repair tissue.

Our study adds to the current body of literature, that the reaction to short bout of exercise has not been tested among a paediatric population so far and the investigation of explicit markers of inflammation are scarce. ${ }^{22}$

Second, following previous findings ${ }^{28-30}$, we expected that a long term AET intervention favorably impacts the inflammatory blood markers in children and adolescents with IBD. Again, this hypothesis was in line with our findings. After a long-term AET intervention ESR values, CRP and thrombocytes significantly decreased in all three groups. However, comparing groups, ESR was still highest in IBD-AD, followed by IBD-RE and HC. Concerning interactions, decreased values for ESR, CRP and thrombocytes were observed over time in IBD-AD and IBD-RE, while values for $\mathrm{HC}$ increased.

Overall, decreased ESR, CRP and thrombocytes are indicator for decreased inflammation and previous studies confirmed the anti-inflammatory effect of exercise in IBD. ${ }^{19,33,70}$ However, the available data did not, unfortunately, shed light on the physiological mechanisms producing the impressive improvements we observed with respect to explicit markers of inflammation. Consequently, we need to draw from ideas of previous studies about possible mechanisms; however three different theories might be at play:

First, exercise reduces transient stool time and on this way reduces the contact time of pathogens with the gastrointestinal mucus layer and circulatory system. ${ }^{23}$ Furthermore, exercise is known to reduce visceral fat mass, subsequently reduces the release of adipokines and introduces an anti-inflammatory environment. ${ }^{71}$ In more detail, Bilski et al. ${ }^{23}$ argued that IBD patients could experience anti-inflammatory effects due to myokines released during skeletal muscle contraction in exercise, which inhibited the release of pro-inflammatory mediators. Myokine, as for example IL-6, are cytokine that are produced and released during skeletal muscle contraction. Further, IL-6 stimulated the production of anti-inflammatory factors (IL-1 antagonist and IL-10) and inhibited pro-inflammatory cytokine (TNF-alpha). ${ }^{23,72}$ On this way, myokines may balance or counteract the effects of proinflammatory stimuli in IBD. Furthermore, they may inhibit the release of proinflammatory mediators from the mesenteric white adipose tissue (mWAT), which is typically deregulated in IBD. ${ }^{23}$ The theory of increased cytokines like IL6 can be connected to our inflammation markers CRP and BSR, since the occurrence of IL-6 strongly correlates with CRP and previous studies recommended that serum CRP is an appropriate marker to measure inflammation in human studies. ${ }^{73}$

Second, Chen and Noble ${ }^{74}$ hypothesized that exercise is a biologically relevant mean to induce protective heat shock proteins (HSPS) for the regulation of 
intestinal inflammation and immunity in animal models. HSPS are known to stabilize denatured proteins, suppress pro-inflammatory transcription factors and decrease the secretion of pro-inflammatory cytokines. ${ }^{75,76}$ However, up to now, it has not been tested, whether exercise induces HPSP increase in the intestine.

Third, evidence emerges how exercise may influence the human gut microbiota. In animal studies, the gut microbiota seems to be sensitive to homeostatic and physiological changes induces by exercise and was associated with an enriched bacterial diversity and activity, which again has been associated with improved health status and alterations in the immune system. ${ }^{77}$ In human studies, first results are diverse: In a study by Clarke et al. ${ }^{78}$ investigating of Rugby players, he reported and association between on the one side high levels of activity and high levels of protein intake and on the other, increased microbiota diversity and decreased state of inflammation. This was in line with Paulsen et al. ${ }^{79}$ who investigated the association between activity levels and microbiota diversity in breast cancer survivors. In contrary, Bressa et al. ${ }^{80}$ did find an association in the opposite direction in a group of health active women vs sedentary women. In the context of IBD, microbiota related complications, such as the leaky gut phenomenon are very frequent and just recently shift into focus. Therefore, the potential effect of exercise on microbiota should be monitored and investigated in IBD more closely.

In summary, current literature points towards an anti-inflammatory effect of exercise, however the exact underlying mechanism remains to be determined. The strength of the present study is that it seems to be the first to investigating the effects of a long-term AET intervention in a paediatric group of IBD-patients. Furthermore, our findings indicate, that clinical monitoring of PA behavior in paediatric IBD may serve as a surrogate outcome parameter for quality of life and inflammation. Consequently, we propose to encourage children and adolescents with IBD to practice moderate PA, since our data supports the hypothesis, that exercise may potentially aid in inflammation suppression and disease management in paediatric IBD. However, the optimal training paradigm (sufficient efficacy without adverse effects on disease activity) for youth with IBD remains to be determined.

Despite the clarity of the results, several issues warrant against overgeneralization. First, the sample size was rather small, though we calculated effect sizes, which are insensitive to sample sizes. Second, no non-interventional control group was included; this limited the potential of the study to detect intervention effects. Third, in this study active vs. non-active IBD was in focus, however, the different etiologies of CD vs UC might involve different mechanisms and should be investigated in future. Fourth, even though we tried to cover an extensive range of factors, it is also conceivable that the pattern of results might have emerged due to further latent, but unassessed physiological variables. Fifth, 
given the nature and structure of the intervention, patients were not unaware of the study condition and therefore we cannot rule out the possibility that treatment effects were due to participants' expectations and motivations. In this context, one suggestion for future studies is to design randomized controlled trials with non-interventional control groups, using larger samples, compare CD vs UD and investigating the effectiveness of different exercise intensities.

\section{Conclusion}

While acute moderate PA enhances inflammatory markers, light to moderate PA for 8 consecutive weeks impacted positively on inflammatory markers in children and adolescents with IBD. Children and adolescents with IBD should be encouraged to practice moderate PA that may aid in inflammation suppression and disease management in paediatric IBD. However, the optimal training paradigm (sufficient efficacy without adverse effects on disease activity) for paediatric IBD remains to be determined.

\section{Acknowledgements}

We thank the Freiwillige Akademische Gesellschaft Basel (FAG. Basel, Switzerland) to financially support the project. Further, we thank the Kantonsspital Aarau to offer additional recruitment options, as well as Noe Stoll for supporting data collection and data entry. Finally, they thank Nick Emler (Surrey, UK) for proofreading the manuscript. 


\section{References}

1 Levine A. de Bie $\mathrm{Cl}$. Turner D. et al. Atypical disease phenotypes in pediatric ulcerative colitis: 5-year analyses of the EUROKIDS Registry. Inflamm Bowel Dis. 1-8 (2012).

2 Lemberg DA. Day AS. Crohn disease and ulcerative colitis in children: An update for 2014. Journal of Paediatrics and Child Health 51. 266-270

3 Dutta. A. K. Influence of environmental factors on the onset and course of inflammatory bowel disease. World J. Gastroenterol. 22. 1088 (2016).

4 Loddo I. \& Romano C. Inflammatory Bowel Disease: Genetics. Epigenetics. and Pathogenesis. Front. Immunol. 6. (2015).

5 Rabizadeh S. \& Dubinsky M. Update in Pediatric Inflammatory Bowel Disease. Rheum. Dis. Clin. N. Am. 39. 789-799 (2013).

6 Cosnes J. Gower-Rousseau C. Seksik P. Cortot A. Epidemiology and natural history of inflammatory bowel diseases. Gastroenterology 140. 1785-94 (2011).

7 Molodecky NA. Soon IS. Rabi DM. Ghali WA. Ferris M. Chernoff G. Benchimol El. Panaccione R. Ghosh S. Barkema HW. Kaplan GG. Increasing incidence and prevalence of the inflammatory bowel diseases with time. based on systematic review. Gastroenterology 142. $46-54$ (2012).

8 Braegger CP. et al. Epidemiology of inflammatory bowel disease: is there a shift towards onset at a younger age? J. Pediatr. Gastroenterol. Nutr. 53. 141-144 (2011).

9 Sanchez-Munoz F. Dominguez-Lopez A. Yamamoto-Furusho JK. Role of cytokines in inflammatory bowel disease. World J Gastroenterol. 14. 4280-8 (2008).

10 Rizzo A. Pallone F. Monteleone G. \& Fantini MC. Intestinal inflammation and colorectal cancer: A double-edged sword? World J Gastroenterol 17. 3092-3100 (2011).

11 Marcus SB. Strople JA. Neighbors K. Weissberg-Benchell J. Nelson SP. Limbers C. Varni JW. Alonso EM. Fatigue and health-related quality of life in pediatric inflammatory bowel disease. Clin Gastroenterol Hepatol. 7. 554-61 (2009).

12 Greenley RN. Hommel KA. Nebel J. Raboin T. Li S-H. Simpson P. \& Mackner L. A Meta-analytic Review of the Psychosocial Adjustment of Youth with Inflammatory Bowel Disease. Journal of Pediatric Psychology 35. 857-869 (2010).

13 Mikocka-Walus A. Knowles SR. Keefer L \& Graff L. Controversies Revisited: A Systematic Review of the Comorbidity of Depression and Anxiety with Inflammatory Bowel Diseases. Inflamm. Bowel Dis. 22. 752-762 (2016).

14 Werkstetter KJ. et al. Lean body mass. physical activity and quality of life in paediatric patients with inflammatory bowel disease and in healthy controls. J. Crohns Colitis 6. 665-673 (2012).

15 Day A. Bishop J. \& Lemberg. D. Managing inflammatory bowel disease in adolescent patients. Adolesc. Health Med. Ther. 1 (2014). doi:10.2147/AHMT.S37956

16 Emmrich J. Conservative standard therapy for patients with Crohn's disease. Chir Gastroenterol 325-30 (2002).

17 Xu CT. Meng SY. Pan BR. Drug therapy for ulcerative colitis. World J Gastroentero 2311-7 (2004).

18 Ertek S. Cicero A. Impact of physical activity on inflammation: effects on cardiovascular disease risk and other inflammatory conditions. Arch Med Sci. 8. 794-804 (2012).

19 Ploeger. H. et al. Exercise and Inflammation in Pediatric Crohn's Disease. Int. J. Sports Med. 33. 671-679 (2012).

20 Gerber. M.. Lindwall. M.. Lindegård. A.. Börjesson. M. \& Jonsdottir. I. H. Cardiorespiratory fitness protects against stress-related symptoms of burnout and depression. Patient Educ. Couns. 93. 146-152 (2013).

21 Gerber M1. Endes K. Herrmann C. Colledge F. Brand S. Donath L. Faude O. Pühse U. Hanssen H. Zahner L. Fitness. Stress. and Body Composition in Primary Schoolchildren. Med Sci Sports Exerc. 49. 581-587 (2017). 
22 Bilski J. Mazur-Bialy Al. Wierdak M. \& Brzozowski T. The impact of physical activity and nutrition on inflammatory bowel disease: the potential role of cross talk between adipose tissue and skeletal muscle. Journal of Physiology and Pharmacology: An Official Journal of the Polish Physiological Society 2. 143-155 (2013).

23 Bilski J. Mazur-Bialy A. Brzozowski B. Magierowski M. Zahradnik-Bilska J. Wójcik D. Magierowska K. Kwiecien S. Mach T. Brzozowski T. Can exercise affect the course of inflammatory bowel disease? Experimental and clinical evidence. Pharmacol Rep 68. 827-36 (2016).

24 Monda V. Villano I. Messina A. Valenzano A. Esposito T. Moscatelli F. Viggiano A. Cibelli G. Chieffi S. Monda M. Messina G. Exercise Modifies the Gut Microbiota with Positive Health Effects. Oxid Med Cell Longev. 3831972. (2017).

25 Slattery ML. Physical activity and colorectal cancer. Sports Med 34. 239-52 (2004).

26 Boot AM. Bouquet J. Krenning EP. de Muinck Keizer-Schrama SMPF. Bone mineral density and nutritional status in children with chronic inflammatory bowel disease. Gut 188-94 (1998).

27 D'Incà R. Varnier M. Mestriner C. Martines D. D'Odorico A. Sturniolo GC. Effect of moderate exercise on Crohn's disease patients in remission. Ital J Gastroenterol Hepatol. 31. 205-10 (1999).

28 Shephard R. The Case for Increased Physical Activity in Chronic Inflammatory Bowel Disease: A Brief Review. Int. J. Sports Med. 37. 505-515 (2016).

29 Narula N. Fedorak RN. Exercise and inflammatory bowel disease. Canadian Journal of Gastroenterology 22. 497-504 (2008).

30 Packer N. Hoffman-Goetz L. Ward G. Does physical activity affect quality of life. disease symptoms and immune measures in patients with inflammatory bowel disease? A systematic review. J Sports Med Phys Fitness. 50. 1-18 (2010).

31 Elsenbruch S. Langhorst J. Popkirowa K. Müller T. Luedtke R. Franken U. Paul A. Spahn G. Michalsen A. Janssen OE. Schedlowski M. Dobos GJ. Effects of mind-body therapy on quality of life and neuroendocrine and cellular immune functions in patients with ulcerative colitis. Psychother Psychosom 74. 277-87 (2005).

32 Gupta N. Khera S. Vempati RP. Sharma R. Bijlani RL. Effect of yoga based lifestyle intervention on state and trait anxiety. Indian J Physiol Pharmacol. 50. 41-7 (2006).

33 Loudon CP. Corroll V. Butcher J. Rawsthorne P. Bernstein CN. The effects of physical exercise on patients with Crohn's disease. Am J Gastroenterol 94. 697-703 (1999).

34 Klare P. Nigg J. Nold J. Haller B. Krug AB. Mair S. Huber W. The impact of a ten-week physical exercise program on health-related quality of life in patients with inflammatory bowel disease: A prospective randomized controlled trial. Digestion 91. 239-247 (2015).

35 Ng SC. Tang W. Leong RW. Chen M. Ko Y. Studd C. Sung JJY. Environmental risk factors in inflammatory bowel disease: a population-based case-control study in Asia-Pacific. Gut 64. 1063-1071 (2015).

36 Daley AJ. Grimmett C. Roberts L. Wilson S. Fatek M. Roalfe A. Singh S. The effects of exercise upon symptoms and quality of life in patients diagnosed with irritable bowel syndrome: a randomised controlled trial. Int $j$ Sports Med 29. 778-82 (2008).

37 Johannesson E. Simrén M. Strid H. Bajor A. Sadik R. Physical activity improves symptoms in irritable bowel syndrome: a randomized controlled trial. Am J Gastroenterol 106. 915-22

38 Robinson RJ1. Krzywicki T. Almond L. al-Azzawi F. Abrams K. lqbal SJ. Mayberry JF. Effect of a low-impact exercise program on bone mineral density in Crohn's disease: a randomized controlled trial. Gastroenterology 115. 36-41 (1998).

$39 \mathrm{Ng} \mathrm{V}$. Millard W. Lebrun C. Howard J. Low-intensity exercise improves quality of life in patients with Crohn's disease. Clin J Sport Med. 17. 384-8 (2007).

40 Taylor RW. Jones IE. Williams SM. \& Goulding A. Evaluation of waist circumference. waist-tohip ratio. and the conicity index as screening tools for high trunk fat mass. as measured by dualenergy X-ray absorptiometry. in children aged 3-19 y. Am. J. Clin. Nutr. 72. 490-495 (2000). 


\section{Chapter 6}

41 WHO Multicentre Growth Reference Study Group. WHO Child Growth Standards: Length/heightfor-age. weight-for-age. weight-for-length. weight-for-height and body mass index-for-age: Methods and development. (2006).

42 Turner D. Griffiths AM. Walters TD. Seah T. Markowitz J. Pfefferkorn M. Keljo D. Otley A. Leleiko NS. Mack D. Hyams J. Levine A. Appraisal of the pediatric Crohn's disease activity index on four prospectively collected datasets: recommended cutoff values and clinimetric properties. Am J Gastroenterol. 105. 2085-92 (2010).

43 Craig CL. et al. International Physical Activity Questionnaire: 12-Country Reliability and Validity: Med. Sci. Sports Exerc. 35. 1381-1395 (2003).

44 IPAQ. Guidelines for Data Processing and Analysis of the International Physical Activity Questionnaire (IPAQ) - Short and Long Forms. revised on November 2005. (2005).

45 Hagströmer M. et al. Concurrent validity of a modified version of the International Physical Activity Questionnaire (IPAQ-A) in European adolescents: The HELENA Study. Int. J. Obes. 32. S42S48 (2008).

46 Meltzer LJ.. Hiruma LS. Avis K. Montgomery-Downs H. \& Valentin J. Comparison of a Commercial Accelerometer with Polysomnography and Actigraphy in Children and Adolescents. SLEEP (2015). doi:10.5665/sleep.4918

47 Kooiman. TJM. et al. Reliability and validity of ten consumer activity trackers. BMC Sports Sci. Med. Rehabil. 7. (2015).

48 Ulrich S. Hildenbrand FF. Treder U. Fischler M. Keusch S. Speich R. \& Fasnacht M. Reference values for the 6-minute walk test in healthy children and adolescents in Switzerland. BMC pulmonary medicine 13. 1 (2013).

49 Hassan J. van der Net J. Helders PJM. Prakken BJ. \& Takken T. Six-minute walk test in children with chronic conditions. Br. J. Sports Med. 44. 270-274 (2010).

50 Borg G. Borg's perceived exertion and pain scales Champaign. viii. (Human Kinetics. 1998).

51 Bailey. BW. Energy Cost of Exergaming: A Comparison of the Energy Cost of 6 Forms of Exergaming. Arch. Pediatr. Adolesc. Med. 165. 597 (2011).

52 Maddison. R. et al. Effects of active video games on body composition: a randomized controlled trial. Am. J. Clin. Nutr. 94. 156-163 (2011).

53 Maddison. R. et al. Active video games: the mediating effect of aerobic fitness on body composition. Int J Behav Nutr Phys Act 9. 54 (2012).

54 Christison A. Khan HA. Exergaming for health: a community-based pediatric weight management program using active video gaming. Clin Pediatr (Phila) 51. 382-8 (2012).

55 Mills. A. et al. The Effect of Exergaming on Vascular Function in Children. J. Pediatr. 163. 806810 (2013).

56 Murphy. E. C.-S. et al. Effects of an exercise intervention using Dance Dance Revolution on endothelial function and other risk factors in overweight children. Int. J. Pediatr. Obes. 4. 205214 (2009).

57 Peng W. Lin J-H. Crouse J. Is playing exergames really exercising? A meta-analysis of energy expenditure in active video games. Cyberpsychology. Behavior and Social Networking 14. 681688 (2011).

58 Quinn M. Introduction of active video gaming into the middle school curriculum as a school-based childhood obesity intervention. Journal of Pediatric Health Care: Official Publication of National Association of Pediatric Nurse Associates \& Practitioners 27. 3-12 (2013).

59 Schoech D. Boyas JF. Black BM. Elias-Lambert N. Gamification for Behavior Change: Lessons from Developing a Social. Multiuser. Web-Tablet Based Prevention Game for Youths. J of Technology in Human Services 31. 197-217 (2013).

60 Bandura A. Self-efficacy: Toward a unifying Theory of behaviour Change. Psychological Review 84. 191-215 (1977).

61 Tanaka H. Monahan KD. Seals DR. Age-predicted maximal heart rate revisited. Journal of the American College of Cardiology 37. 153-156 (2001). 
62 Cohen. J. Statistical Power Analysis for the Behavioral Sciences (2 edition). (Hillsdale. N.J: Routledge. 1988).

63 Hlavaty T. Toth J. Koller T. Krajcovicova A. Oravcova S. Zelinkova Z. and Huorka M. Smoking. breastfeeding. physical inactivity. contact with animals. and size of the family influence the risk of inflammatory bowel disease: A Slovak case-control study. United European Gastroenterol J 1. 109-119 (2013).

64 Tew GA. Jones K. Mikocka-Walus A. Physical Activity Habits. Limitations. and Predictors in People with Inflammatory Bowel Disease: A Large Cross-sectional. Inflamm Bowel Dis. 22. 29332942. (2016).

65 Natale VM. Brenner IK. Moldoveanu AI. Vasiliou P. Shek P. Shephard RJ. Effects of three different types of exercise on blood leukocyte count during and following exercise. Sao Paulo Med J. 121. 9-14 (2003).

66 Sand KL. Flatebo T. Andersen MB. Maghazachi AA. Effects of exercise on leukocytosis and blood hemostasis in 800 healthy young females and males. World J Exp Med. 3. 11-20 (2013).

67 Kaspar F. Jelinek HF. Perkins S. Al-Aubaidy HA. deJong B. Butkowski E. Acute-Phase Inflammatory Response to Single-Bout HIIT and Endurance Training: A Comparative Study. Mediators Inflamm. 2016:5474837. (2016).

68 Simpson RJ1. Kunz H2. Agha N2. Graff R2. Exercise and the Regulation of Immune Functions. Prog Mol Biol Transl Sci 135. 355-80 (2015).

69 Berg JM. Tymoczko JL. Stryer L. Biochemistry. 5th edition. (W H Freeman. 2002).

70 Chan. D.. Robbins. H.. Rogers. S.. Clark. S. \& Poullis. A. Inflammatory bowel disease and exercise: results of a Crohn's and Colitis UK survey. Frontline Gastroenterol. flgastro-2013 (2013).

71 Gleeson M1. Bishop NC. Stensel DJ. Lindley MR. Mastana SS. Nimmo MA. The anti-inflammatory effects of exercise: mechanisms and implications for the prevention and treatment of disease. Nat Rev Immunol. 11. 607-15 (2011).

72 Pedersen BK. Febbraio MA. Muscles. exercise and obesity: skeletal muscle as a secretory organ. Nat Rev Endocrinol 8. 457-65 (2012).

73 Il'yasova D. Ivanova A. Morrow JD. Cesari M. Pahor M. Correlation between two markers of inflammation. serum C-reactive protein and interleukin 6. and indices of oxidative stress in patients with high risk of cardiovascular disease. Biomarkers 13. 41-51 (2008).

74 Chen. Y. \& Noble. E. G. Is exercise beneficial to the inflammatory bowel diseases? An implication of heat shock proteins. Med. Hypotheses 72. 84-86 (2009).

75 Chen Y. Ross BM. Currie RW. Heat shock treatment protects against angiotensin II-induced hypertension and inflammation in aorta. Cell Stress Chaperones 9. 99-107 (2004).

76 Chen Y. Currie RW. Small interfering RNA knocks down heat shock factor-1 (HSF-1) and exacerbates pro-inflammatory activation of NF-kappaB and AP-1 in vascular smooth muscle cells. Cardiovasc Res. 69. 66-75 (2006).

77 Bermon S. Petriz B. Kajènienè A. Prestes J. Castell L. Franco OL. The microbiota: an exercise immunology perspective. Exerc Immunol Rev 70-9 (2015).

78 Clarke SF. Murphy EF. O'Sullivan O. Lucey AJ. Humphreys M. Hogan A. Hayes P. O'Reilly M. Jeffery IB. Wood-Martin R. Kerins DM. Quigley E. Ross RP. O'Toole PW. Molloy MG. Falvey E. Shanahan F. Cotter PD. Exercise and associated dietary extremes impact on gut microbial diversity. Gut, 63(12):1913-20 (2014).

79 Paulsen JA. Ptacek TS. Carter SJ. Liu N. Kumar R. Hyndman L. Lefkowitz EJ. Morrow CD. Rogers LQ. Gut microbiota composition associated with alterations in cardiorespiratory fitness and psychosocial outcomes among breast cancer survivors. Support Care Cancer 25. (2017).

80 Bressa C. Bailén-Andrino M. Pérez-Santiago J. González-Soltero R. Pérez M. Montalvo-Lominchar MG. Maté-Muñoz JL. Domínguez R. Moreno D. Larrosa M. Differences in gut microbiota profile between women with active lifestyle and sedentary women. PLOS ONE 12. (2017). 



\section{Part 4 \\ Bringing Innovation to the Market}

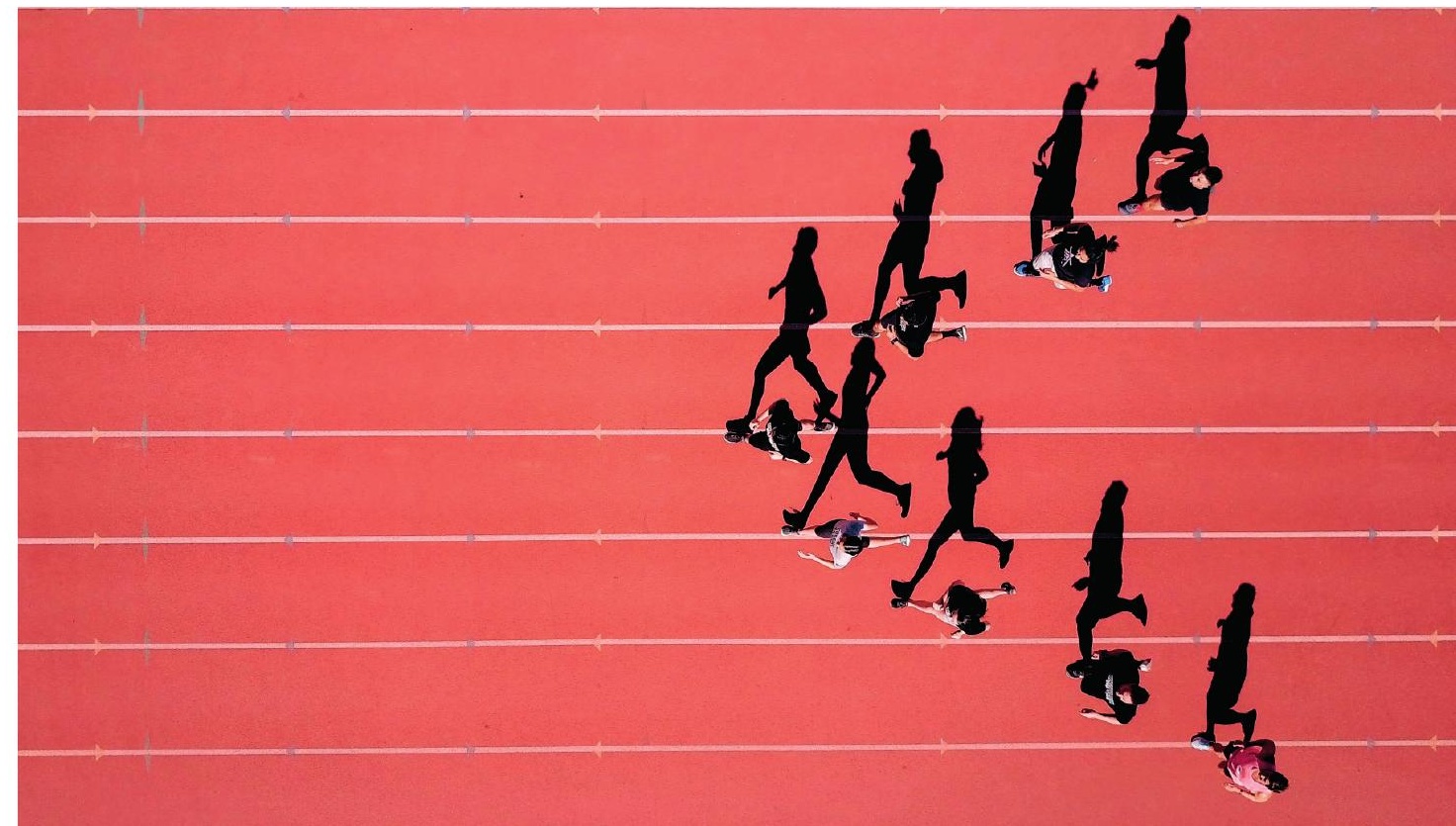


Part 4 
Bringing innovative PM solutions to the market presents a new set of challenges, including the issue of uncertainty. There will be opportunities to support the development of new risk-based approaches for the evaluation of PM in a context that encourages systematic early dialogue with all stakeholders, including regulators, funders and innovators, providing guidance for companies to enter the market for PM. [...]

SRIA, Challenge 4 [38]

A crucial tool in terms of innovation and development of PM is mobile health (mHealth). The constantly increasing computing power and mobile connectivity in our pockets form a great opportunity towards personalized prevention and personalized maintenance $[107,108]$. Today's smartphones incorporate the computational power, that not too long ago would have cost millions of dollars and required room-sized instruments [109]. According to the Ericsson Mobility Report in 2017, 5.3 billion unique mobile subscribers have been identified globally (fig. 3) [109].

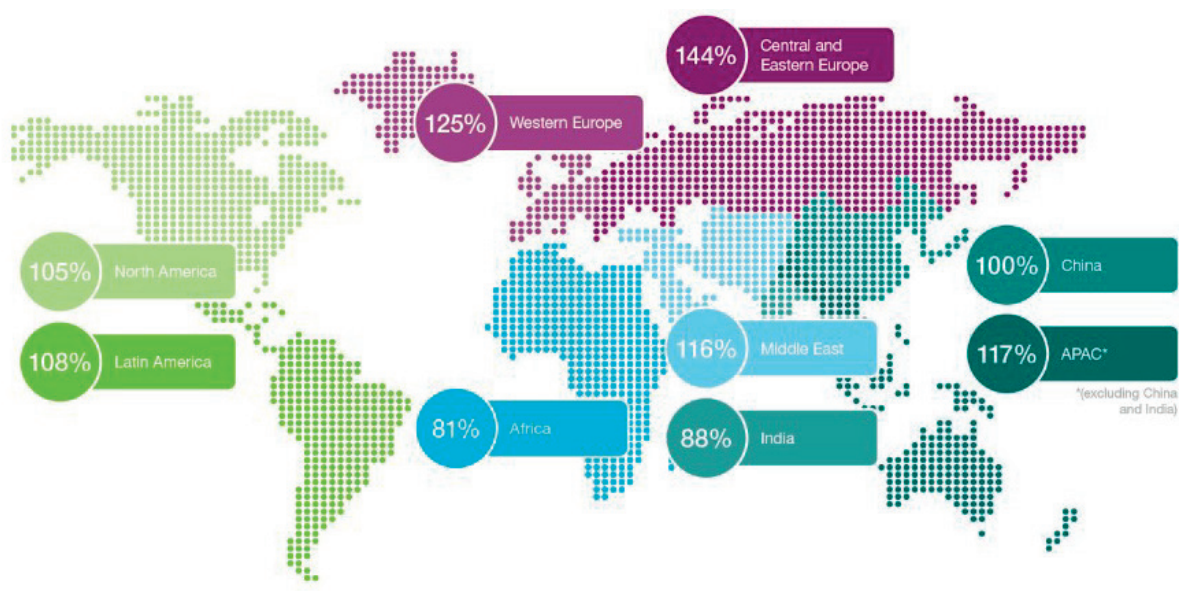

Ericsson Mobility Report November 2017

Figure 3: Subscription penetration 2017 (percent of the population) [109]

Overall, the smart phone has become a substantial part of daily life and people spend increasing amounts of time with their personal computer to manage, connect and entertain themselves. Besides that, this geographically independent device, equipped with sensors, all built around real-time data streams, has the capability to enable care and could support action against critical health challenges of the current era and support the realization of preventive medicine [110].

MHealth, as the practice of medicine supported by mobile devices, health apps and wearable technology, represent a fast changing field of innovation, as exemplified by the rising number of applications flooding the market. Many 


\section{Part 4}

companies have picked up the opportunity of health apps [111]. In the context of epidemics of non-communicable diseases in western societies, and considering the strong influence of lifestyle related risk factors, apps appear to be an ideal platform to deliver simple and effective (health) interventions [112].

The convergence of wearable electronics, miniaturized sensor technologies, and mobile phone penetration provides novel opportunities for personalized lifestyle interventions and support of health care practice. For example, the positive effects of regular physical activity are well known, yet many people do not comply with physical activity guidelines [113]. General practitioners and other medical professionals often don't have time to encourage inactive patients or personalize their lifestyle advice to the patient's needs [114]. As soon as people start moving, it is important to maintain this behavior. The continuation or persistence of such health practices is a second barrier that many people fail to surpass [116]. Physical activity behavior is highly dynamic, momentary and embedded in context. To fill the gap of knowledge and support the practical integration of lifestyle into the clinical context, the following objective has been defined:

Objective (4): To design a tool that will translate scientific evidence on behavior change into a personalized program to support GPs and empower patients to start a physically active life.

This objective has been addressed in a business plan [117], presenting the innovative idea of "MoVit". This business plan has been pitched at multiple start-up events in the Netherlands and Switzerland. 


\section{Chapter 7}

Movit

Published as: Mählmann L: MoVit - ICT 4 Good Fellowship Application 2016 


\section{Chapter 7}

\section{Abstract}

The positive effects of regular physical activity are well known, yet many people do not comply with PA guidelines. GPs and other medical professionals often don't have time to encourage inactive patients or personalize their lifestyle advice to the patients' needs. As soon as people start moving, it is important to maintain this behavior, a second barrier many people fail. MoVit translates scientific evidence of behaviour change into a personalized physical activity coachings program and mobile application to provide health care professionals with a tool to promote an active lifestyle among their patients. 


\section{MOVif}

Mini Business Plan - ICT 4 Good Fellowship

Written by Laura Mählmann (laura.maehlmann@gmail.com, +41 77444 4780)

The positive effects of regular physical activity are well known, yet many people do not comply with PA guidelines. GPs and other medical professionals often don't have time to encourage inactive patients or personalize their lifestyle advice to the patients' needs. As soon as people start moving, it is important to maintain this behavior, a second barrier many people fail.

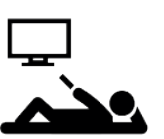

In Switzerland, inactivity causes at least 2,900 premature deaths per year, 2.1 million cases of illness, and direct treatment costs totaling CHF 2.4 billion, while approximately $15-20 \%$ of society are inactive.

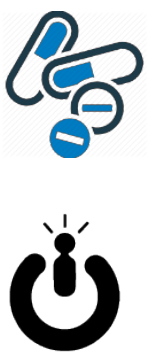

Bad lifestyle, too frequent application of a diagnostic label and overuse of medication lead to increasing health care costs and a decreasing quality of life.

Rising movement to empower and educate patients, find personalized treatment and facilitate with mobile technology: Consumers want technology to help them exercise smarter at a low price, highest trust with the primary health care professional. To realize its full potential, mobile technology in healthcare has to cross the boundary from consumer electronics devices to regulated medical devices.

\section{MOVil}

translates scientific evidence of behaviour change into a personalized physical activity coachings program and mobile application to provide health care professionals with a tool to promote an active lifestyle among their patients.

\section{Benefits}

We are based on science MOVit is based on the MoVo Program, which integrates central elements of social cognition research, with its forces on motivational aspects and self-regulation research with its emphasis on the volitional side of behavioral control. 


\section{Chapter 7}

We support people to become more active The positive health effcts of physical activity are well documented: Epidemiological and clinical studies show that physical activity reduces risks for multiple disease.

We empower people Every participant will be empowered by our program to become more active, will take over health ownership, will be supported by a robust social network and provided with personalized (technical) tools.

We interact with healthcare MOVit enables the health professional to prescribe organized lifestyle changes and preventive treatment and personal activity reports provide an additional continous source of information for medical decision making.

We create new knowledge Our learning system is evidence based and grounded in research and experience we obtain and valuable insights will contribute to new scientific research.

We make it a game During the programm participants will be able to collect points, reach certificates which can be shared in the social network and receive discounts and coupons with our partners.

We help to save costs Movit has major cost-benefit advantages due to prevention of disease. For consumers it is an affordable one-time investment that will facilitate long term behaviour change.

\section{Causal Relationships}

MoVo is a validated behavior change model and multiple scientific reports proof the effectiveness of the MoVo behavior change model.With app supported follow up we expect even improved outcomes and compliance, since scientific studies confirm the value of technological applications to improve quality of care (more data - better treatment), access to care (health coach for everybody), health outcomes (movement benefits health), patient engagement (contribute to becoming healthy, take healthy choices, educate patients, health literacy, more targeted care), chronic disease management, quality of life.

Impact in 1 - 3 years

Health \& Social: All stakeholders will benefit by the increasing awareness of the importance of physical activity among inactive people and the accompanied health benefits. Second, health professionals will be enabeled prevent worsening their disease by prescribing preventive measures. Measurement: In the first three years we want to underlie these assumptions with facts in different patient groups 
of our pilot projects by measuring health outcomes, physical activity behavior, quality of life and patient professional interaction.

Economic: Prevention of adverse health outcomes may have an enourmous impact on future health care budgets, since these are strongly associated with mortality and long-term morbidity. By reducing the total number of inactive patients we can significantly reduce direct treatment costs of CHF 2.4 billion. Measurement: In our pilots we will evaluate days of work absenteism, quality of live and treatment costs compared to a control group.

Ecological: By offering an new channel for research participation and enable ecological momentary assessments of patients without the need of self reporting for a specific study, we facilitate an ecological way of data acquiration for research. Measurement: Amount of collabrating partners and successful pilot projects and planned research projects.

\section{Business model}

With health professionals as out first partners, we create an access channel to the target population and provide the health care professional with a tool to prescribe preventive measures. The business model will be introduced stepwise. First we start to offer the program in different packages to patients transferred by doctor and advised to participate in the program due to diagnosed inactivity or in revalidation. This will target early adopters, patients who see a great value and personal benefit in an intensively guided activity coaching program.

Cost packages:

\begin{tabular}{lll}
\hline 1. MoVo Program \& Material & $250 \mathrm{CHF}$ \\
2. App & $5 \mathrm{CHF}$ \\
3. MoVo Program and App & $255 \mathrm{CHF}$ \\
4. Follow up face to face coaching & $40 \mathrm{CHF}$ \\
5. Advanced App Version & $10 \mathrm{CHF}$ \\
\hline
\end{tabular}

Second we want to partner with health care insurances to develop a pay-back system after successful completion of the program. This will lower the financial barrier for patients to participate and create an incentive to complete the entire program. Third we are looking for partners like sport brands, health and prevention initives and health insurances to advertise via our program and provide rewards like coupons and discounts for special accomplishments in the program. Finally, we collaborate with public scientific institutions to set up research projects based on our data. 


\section{Chapter 7}

\section{Value Proposition}

Patients receive the chance to take ownership of his/her health and actively engage with his/her wellbeing. Encouraged and monitored by health professionals and coaches people receive personalized tools and techniques to overcome barriers and reach their goals successfully suited to their persons specific needs. Finally the program enourages people to increase activity in their daily life by suggesting small but significant changes which only cost very small effort. For parties aiming to improve people's health the program provides a promising tool to encourage and monitor people to become more active. Finally the product will become a medical devise contributing to the revolutionary development: Apps instead of pills. Compared to our competitors we combine all factors needed for successful behaviour change and base our tools on a validated behaviour change model. To realize its full potential we collaborate with health professionals, governmental instiutions and the private sector. Finally, we do provide people with suggestions for small changes they can easily implement in daily live. For us it is important to personalize the intervention and find out what ways of activity are suitable and most simple to implement in the persons individual context.

\section{Market Size and Opportunity}

To estimate market size we adhere to numbers of inactivity in Switzerland proposed by the Swiss health Survey. In this study, a person is considered as physically inactive, if she does not meet the following recommendations for health enhancing physical activity: at least 2.5 hours of physical activity with moderate intensity per week or 1.25 hours of sports with high intensity per week.

The mean percentage of inac-

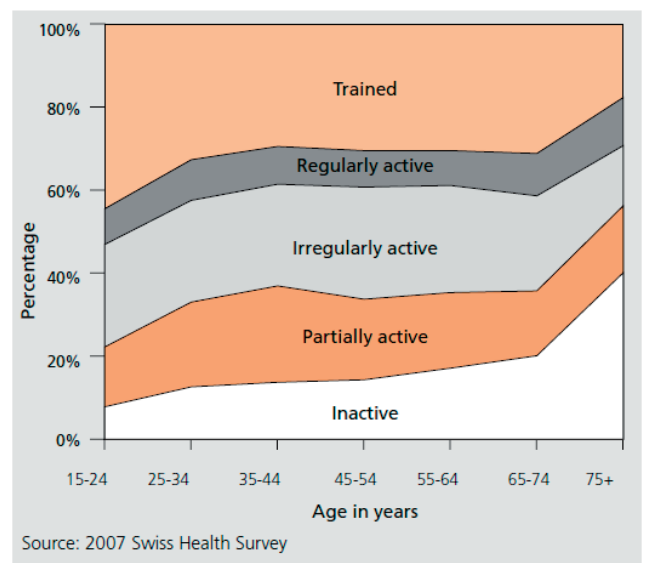
tivty for the age groups 15 years + in Switzerland is $27.5 \%$, while more prevalent in women. Since after an adverse life event patients are most accesible for behaviour change and highly motivated to improve their condition, we will focus on this target population.

\section{Benchmarking}

Smartphones and smartphone applications offer new possibilities for promoting PA. The rapidly growing number of fitness apps that are commercially available 
indicate their popularity. Well known commercially oriented fitness apps are RunKeeper, Nike +/Nike Training Club, Move, Zombie Run, Fitocracy and many others targeting healthy people and help to increase fitnesslevels by offering tools to track runs, suggest exercises to for strength training or provide entire fitness programs. Furthermore wearable technology like Fitbit, Jawbone and many others help people to track their amount of steps and monitor whether they reach the defined health goal which is most of the time 10000 steps per day.

All of these apps are based on fewer than four behavior change techniques and are either focused on educational or motivational behavior change techniques - people might need multiple apps to initiate and maintain behavior change. Surprisingly, especially the most well established technique for bridging the intention-behavior gap, action planning, is relatively rare in description of the top-ranked physical activity apps.

\section{Stakeholders}

Health care professionals, Patients, Government, Health care insurances and Researchers.

\section{Budget and Finance}

\begin{tabular}{|c|c|c|c|c|}
\hline Costs & per month & 2016 & 2017 & 2018 \\
\hline Development of MVP & \multicolumn{4}{|c|}{3.000} \\
\hline Rent for group meetings locations & sponsored & & & \\
\hline App development & & 50,000 & & \\
\hline Owners compensation & 5,000 & 60,000 & 60,000 & 60,000 \\
\hline Developer salary & 100 & 12,000 & & \\
\hline Coach (1) salary & 2,500 & & 30,000 & 30,000 \\
\hline Coach (2) salary & 2,500 & & & 30,000 \\
\hline Coach (3) salary & 2,500 & & & 30,000 \\
\hline Credit card \& bank charges & 50 & 600 & 600 & 600 \\
\hline Insurance & 300 & 3,600 & 3,600 & 3,600 \\
\hline Communication (Phone, Internet) & 200 & 2,400 & 2,400 & 2,400 \\
\hline Material & 300 & 3,600 & 3,600 & 3,600 \\
\hline Travel expenses & 500 & 6,000 & 6,000 & 6,000 \\
\hline Tax & & 1,000 & 3,000 & 7,000 \\
\hline Licenses & & 1,000 & 1,000 & 1,000 \\
\hline Pilot investment & & 6,000 & & \\
\hline Data warehouse & & & 2,000 & 4,000 \\
\hline Data management & & & 5,000 & 10,000 \\
\hline Advertisment & & 1,000 & 2,000 & 2,000 \\
\hline Total & & 150,200 & 119,200 & 190,200 \\
\hline
\end{tabular}




\begin{tabular}{llrrr}
\hline Revenue Streams & & 2016 & 2017 & 2018 \\
\hline Program (250 CHF) & \# sold & 300 & 1,500 & 3,000 \\
& amount & Free & 375,000 & 750,000 \\
App (5 CHF) & \# sold & 300 & 4,000 & 10,000 \\
& amount & 1,500 & 20,000 & 50,000 \\
Advanced App (10 CHF) & \# sold & 0 & 1,000 & 3,000 \\
& amount & 0 & 10,000 & 30,000 \\
Additional coaching (40 CHF) & \# sold & 30 & 400 & 800 \\
& amount & 1,200 & 16,000 & 32,000 \\
Funding & & 100,000 & 250,000 & 250,000 \\
Sponsoring & & & & 250,000 \\
Total & & $\mathbf{1 0 2 , 7 0 0}$ & $\mathbf{6 7 1 , 0 0 0}$ & $\mathbf{1 , 3 6 2 , 0 0 0}$ \\
\hline
\end{tabular}

Revenue projections are based on the capacity and market outreach and our ability to increase our partner base. 2016 is the year of product launch, pilot and scaling. Therefore we fous on quality, not quantity to find the optimal franchise and distribution model for scaling. In 2017 we expect to reach the break-even point from a sale of approximately 1000 programs. Further, we expect to obtain additional funding by the Swiss Foundation for prevention, different health insurances, funding for innovation in health care by the Swiss government as well as partnering with sports brands like Asics

\section{Founder}

Laura Mählmann has a broad academic background in the field of health science, genomics and big data. She is an open minded and internationally oriented person. She graduated in health science research and global health. She participated in the Dutch National ThinkTank, a Dutch foundation aiming to improve the cooperation and knowledge exchange between the academic, public and private sector, and contribute innovatively to society. During this ThinkTank her scientific interest in innovative concepts for health care and personalized/patient centred health care facilitated by new technology extended to a more entrepreneurial and practical dimension: The ShopBuddy. Inspired by the first entrepreneurial successes with her team in the Netherlands, Laura is compassionate to translate knowledge obtained by research into practical solution to directly benefit society.

Board of Advisors: Prof. Dr. Uwe Pühse, (Co-Founder) Director DSBG University of Basel, Dr. phil Markus Gerber, Assistant Director DSGB University of Basel (Co-Founder), Dr. Phil Serge Brand UPK, Basel, Dr. med. Raoul Furlano (UKBB), Rembrand Satorious (Mc Kinsey \& Company). 


\section{Roadmap \& Impact Hub Fellowship}

\begin{tabular}{|c|c|c|c|}
\hline Mile stone & Details & Outcome & Months \\
\hline $\begin{array}{l}\text { Ideation and } \\
\text { confirmation }\end{array}$ & $\begin{array}{l}\text { Create an investor slide deck, test the concept } \\
\text { among stakeholders and build a network of } \\
\text { partners and investors }\end{array}$ & $\begin{array}{l}\text { Survey } \\
\text { Pilot investor }\end{array}$ & $1-3$ \\
\hline Creation & $\begin{array}{l}\text { Define the app concept, test the offline concept } \\
\text { in small groups and set up a MVP Design a pilot } \\
\text { and recruitment strategy Evaluate the Business } \\
\text { model }\end{array}$ & $\begin{array}{l}\text { MVP } \\
\text { Pilot Plan BM } 2.0\end{array}$ & $1-3$ \\
\hline Validation & $\begin{array}{l}\text { Start a pilot \& evaluation of pilot } \\
\text { Start next iteration cycle }\end{array}$ & Pilot 1 Results & $4-12$ \\
\hline Repeatabilty & New pilots in other population groups & Pilot 2 \& 3 Results & $4-12$ \\
\hline $\begin{array}{l}\text { Business } \\
\text { development }\end{array}$ & $\begin{array}{l}\text { Business Model reworking Funding Strategy } \\
\text { Marketing Strategy } \\
\text { Promotion \& Investor Network Scaling Strategy }\end{array}$ & $\begin{array}{l}\text { Roadmap } 2.0 \\
100.000 \mathrm{CHF} \\
\text { funding }\end{array}$ & $4-12$ \\
\hline
\end{tabular}

\section{Impact hub support}

- Entrepreneurial Expertise to specify and redefine the business model

- Work in an organized and inspirational atmosphere - learn from other startups and entrepreneurs and participate in workshops

- Enlarge the network 



\section{Part 5}

\section{Shaping Sustainable Healthcare}

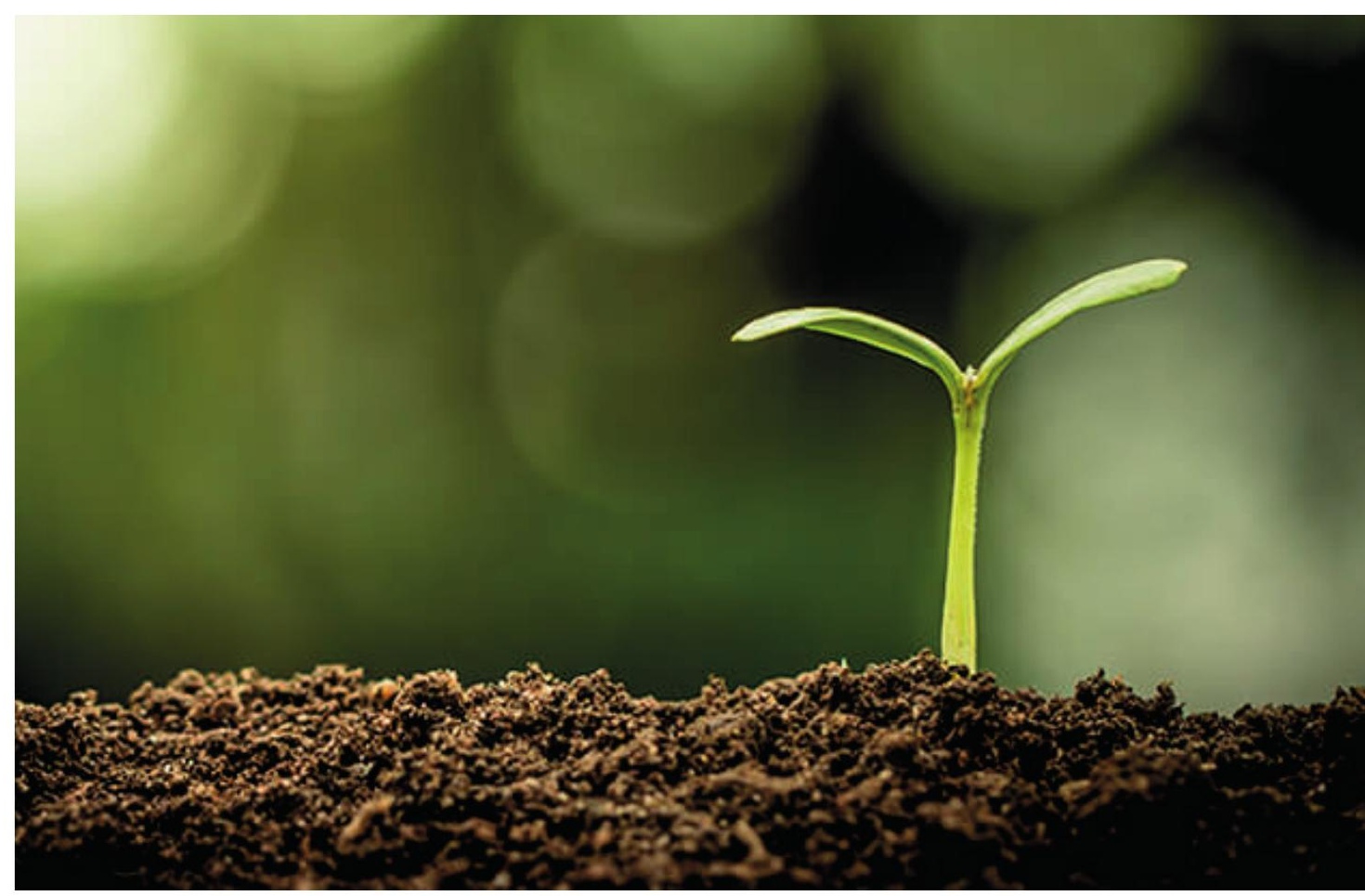



PM needs to rely on a knowledgeable healthcare system that is able to adapt to these new approaches in a timely and socially acceptable way, and that enables the participation of all stakeholders to increase PM's effectiveness and efficiency. [...]

SRIA, Challenge 5 [38]

Ever since the establishment of health care systems, it has been the goal to (1) improve health, both at the absolute level across the entire population and in equal measures across socioeconomic groups, (2) protect the social and financial risk in health, (3) be responsive and people-centered and (4) be highly efficient [118]. The aim and vision of PM raised high expectations and hopes from the perspective of health care systems. With the promise of PM to improve outcomes by providing treatment to the right patient, at the right dosage, at the right time and by avoiding the traditional "one-size fits all" approach based on trial and error, new opportunities emerged to reach the health care systems goals. Understandably, this development is expected to reduce the economic burden that health care systems are facing due to adverse treatment effects, incomplete benefits or misdiagnosis [119].

However, the changing medical, technological and societal conditions also create new demands on the health care systems. Changes in treatment approaches will influence the practices of quality assurance, organizational aspects and capacity building. One concept that will challenge health care systems is the changing classification of diseases into common diseases (CDs) and rare diseases (RDs). To illustrate this scenario in more detail the following objective has been defined:

Objective (5): Discuss the role of health care systems and policy making when shifting focus from traditional disease categorization towards unique disease profiles.

The fifth objective is discussed in the book chapter by Schee gen. Halfmann and Mählmann et al. [121] entitled "Personalized Medicine: What's in it for Rare Diseases?" (Chapter 8). 



\section{Chapter 8 Personalized Medicine: What's in it for Rare Diseases?}

Published as: Schee Genannt Halfmann S, Mählmann L, Leyens L, Reumann M, Brand A. Personalized Medicine: What's in it for Rare Diseases? Adv Exp Med Biol. 2017;1031:387-404. doi: 10.1007/978-3-319-67144-4_22. [IF:1.64] 


\section{Chapter 8}

\section{Abstract}

Personalized Medicine has become a reality over the last years. The emergence of 'omics' and Big data has started revolutionizing healthcare. New 'omics' technologies lead to a better molecular characterization of diseases and a new understanding of the complexity of diseases. The approach of PM is already successfully applied in different healthcare areas such as oncology, cardiology, nutrition and for rare diseases. However, health systems across the EU are often still promoting the 'one-size fits all' approach, even if it is known that patients do greatly vary in their molecular characteristics and response to drugs and other interventions. To make use of the full potentials of PM in the next years ahead several challenges need to be addressed such as the integration of Big Data, patient empowerment, translation of basic to clinical research, bringing the innovation to the market and shaping sustainable healthcare systems. 


\section{Introduction}

Over the last decades medical treatment in Europe has been based on the concept of evidence based medicine (EBM), which intends that decision making in medical practice is informed by the most reliable scientific information combined with individual expertise of the health professional, as well as patient preferences [3]. In practice, patients mainly receive treatments and medication that have been assessed and tested regarding efficiency and safety in well-designed Randomized Clinical Trials (RCTs), the gold standard in clinical research [52]. Nevertheless, this approach does not take into account the individual molecular characteristics of the patients, which are of great importance for the effectiveness and safety of therapies. Patients do not respond to therapies and drugs in the same way $[24,32,49]$ due to differences in genomic and epigenomic profile [36]. Therefore, the traditional approach of EBM has been critizied as an ineffective 'onesize fits all' healthcare approach [32]. Furthermore, patients who receive drugs that do not fit their needs will either continue to carry the burden of the current health condition or even suffer from more severe health problems due to the accompanied side effects such as adverse drug reactions (ADRs). For example, evidence indicated that the 'one-size fits all' approach in cancer treatment is effective in $25 \%$ of the cases [57], however $75 \%$ of cancer therapies and treatments are not effective and patients suffer from ADRs and a loss of quality of life during treatment [57].

Not only do the patients suffer from ineffective treatments or even ADRs, the current approach also results in economic inefficiency of healthcare systems across Europe. In addition to the rising morbidity, demographic changes and the burden of non-communicable diseases (NCDs), the low treatment response rate creates an economic burden of more than EUR 100 billion each year [32].

Another traditional approach in health care is the classification of diseases into common diseases (CDs) and rare diseases (RDs) [45]. In Europe diseases that affectless than 5 patients per 10,000 citizens are defined as rare diseases. Across the EU, approximately 30 million European citizens are suffering from RDs. However, emerging 'omics technologies' which enable sequencing of the human genome have first proven that patients have unique molecular characteristics [33] and second, that each mutation of a tumor is different [55]. Therefore, this new understanding of the complexity of diseases allows to classify diseases more accurate based on their genetic characteristics [44] using next generation sequencing. This results in a new understanding of diseases which makes no differences between CDs or RDs. Finally, according to Boycott et al. [4] the large majority of disease causing gene mutations will be discovered by 2020 .

At this point, the approach of personalised medicine (PM) joins the discussions. Emerging technologies such as Whole Genome Sequencing (WGS), 


\section{Chapter 8}

Whole Exome Sequencing (WES) or Low-Coverage Sequencing (LCS) identified the need for bet- ter understanding of the molecular basis of disease and evened the path for PM. Based on molecular interindividual differences, PM applies an understanding that all diseases become rare diseases due to the uniqueness of each patient. For the purpose of the article, we apply this understanding and we do not differentiate between CDs and RDs.

\section{What Does Personalised Medicine Mean?}

Lately, PM is an often-used buzz word mentioned in discussions regarding healthcare and medicine. PM is an approach that is defined in many different ways among stakeholders and healthcare professionals [18]. Within the literature terms such as genomic medicine, stratified medicine or precision medicine are used interchangeably to describe the approach of PM [55]. Those terms arouse expectations of great medical advances even it is not fully clarified what personalised medicine means.

Since there is no uniform definition of PM, professionals differ in their understanding of the approach of PM, which consequently leads to misunderstandings and miscommunications [53]. While some experts only perceive treatments that are based on genetic analysis and biomarkers as PM [48], others describe PM as an approach in which the healthcare professional bases his treatment decision by taking into account the health status of the patient and the individual circumstances of the patient. In those cases, it is often referred to as individualized medicine [47].

For the purpose of this paper we refer to the definition of PM of the Horizon2020 Advisory Group for Societal Challenge "Health, Demographic Change and Wellbeing" of the European Commission. The Advisory group defines the concept of PM as 'a medical model using characteristics of individuals' phenotypes and genotypes (e.g., molecular profiling, medical imaging, lifestyle data) for tailoring the right therapeutic strategy for the right person at the right time, and/or to determine the predisposition to disease and/or to deliver timely and targeted prevention' [15]. This definition implies the understanding that the healthcare approaches are moving away from the traditional 'one-size fits all' approach.

\section{Potentials of Personalised Medicine}

By adjusting treatments to the unique molecular characteristics of the patients, PM has the potential to make treatments more effective and to decrease the economic burden. In order to identify the biological characteristics of the patient and 
their predispositions to a certain disease, PM applies 'omics technologies' [1,36] such as 'genomics, epigenomics, proteomics, metabolomics, lipidomics' and incorporates real life data of the patients such as environmental and lifestyle information [7,25]. All together, environmental, biological and lifestyle information adds up to an explosion of data soon reaching dimensions of "big data" [2].

PM is already applied successfully in various different healthcare fields [36] and therefore no longer seen as an abstract approach. Over the last decade 'omics technologies' and PM have had the greatest impact on oncology and cancer therapies [55] as well as other medical fields including rare diseases [36, 47], cardiology [59] and also for the treatment of infectious diseases [20].

Nevertheless, there is still potential for healthcare systems across Europe to further strengthen the uptake and implementation of PM [47]. Healthcare systems are not making full use of the potential of PM due to several barriers [25, 33, 47] and prefer to apply the traditional healthcare approach rather than PM. One reason is the complex and slow moving nature of health care systems as well as the lack of illustrating evidence that is needed to demonstrate the benefit of the PM approach.

\section{Personalised Medicine on the European Union Agenda}

As one of the main drivers of PM across Europe and beyond, the European Commission (EC) addresses challenges regarding PM, biobanking and 'omics- technologies' in several reports that have been published since 2013 [13, 14]. Furthermore, over the last 10 years, the European Commission committed around 1 billion Euros of funding to advance 'omics technologies' and PM [14].

One milestone that has been achieved regarding PM, is the launch of the 'Council Conclusions on Personalised medicine for patients' in December 2015 by the Luxembourg Presidency of the Council of the European Union (EU).

Furthermore, with the launch of the 'International Consortium of Personalised Medicine (ICPerMed)' in November 2016, European countries aim to coordinate health research policy to advance the implementation of PM [12]. The initiative brings together the EC, and health research funders and policy making organisations from 28 countries and five regions across Europe and Canada. Within the next few years a roadmap of research action will be defined, which is based on the 'Strategic Research and Innovation Agenda (SRIA)' developed by the EC funded Coordination and Support Action (CSA) PerMed: 'Shaping Europe's Vision for Personalised Medicine' [47]. The CSA PerMed consisted of partners representing European and national key decision makers in research and research policy, healthcare and industry, as well as patient organisations. The PerMed SRIA lists five key challenges and provides 35 recommendations at national and 


\title{
Chapter 8
}

EU level to address those challenges. The following five key challenges for the implementation of PM were identified: 'Developing Awareness and Empowerment, Integrating Big Data and ICT Solutions; Translating Basic to Clinical Research and Beyond, Bridging Innovation to the Market, Shaping Sustainable Healthcare' $[12,47]$.

\section{Challenges to Implement Personalised Medicine into Healthcare Systems}

The five key challenges of the PerMed SRIA need to be tackled and solved to promote the effective, efficient and timely implementation of PM in European healthcare systems in a socially acceptable manner. At the moment, aspects such as the integration of big data, the design of clinical trials, financing and reimbursement mechanisms and the active role of the patient/citizen in the decision-making process need to be addressed [9, 25]. In this book chapter, we present the five PerMed challenges and discuss the impact of PM on rare diseases (RDs). Furthermore, we suggest potential solutions.

\section{Challenge 1 - Developing Awareness and Empowerment}

\begin{abstract}
Successful implementation of PM will be achieved only if all stakeholders, including patients and healthcare professionals, are empowered and develop the required awareness about PM. The crucial first step is to provide the best available evidence that supports the clinical and personal utility of $\mathrm{PM}$, as well as its economic value to health systems, and to enable better understanding of how the changes brought by PM will impact public health for the benefit of individual citizens and society. Models that enable sharing, ownership and the development of a sense of responsibility towards personal health data, as well as the improvement of PM health literacy, will need to be generated along with suitable common principles, appropriate policy and regulatory frameworks [47].
\end{abstract}

Innovative treatments and therapies emerging in the field of PM are often challenging healthcare professionals (HCPs). HCPs feel overwhelmed and overloaded by the amount of new information, tools and technologies, which PM provides, to support their decision making process $[27,36]$. This is due to the fact that across the EU, most of the curricula for HCPs are not up-to-date and do not include the new insights and understandings of the complexity of diseases arising from PM [9, 25, 46]. Issues that are elementary components of PM such as ICT solutions, companion diagnostics, the use of 'omics' technologies are often not 
addressed [36]. Furthermore, great differences and variations occur between the EU Member States and differences exist at national, regional and local level [27]. Thus, there is not only an urgent need to update current curricula to the new understanding of the complexity of diseases to new innovative therapies and diagnostic tools. Of similar importance is that HCPs are also trained in the legal, economic and ethical implications of PM.

Besides the essential role of HCP education in the implementation of PM, patients and citizens are the key stakeholders that need to be empowered and health literate. The patient is often seen as a passive recipient within the healthcare system $[5,43]$. For example, patients are rarely scrutinizing the decisions of their general practitioners (GPs) since they trust in their GPs [5]. To strengthen the uptake of PM it is of great importance to change the role of the patient from a passive recipient to an actively involved stakeholder of the decision-making process of his/her health interventions including prevention, diagnostics and therapies [8, 43, 47]. Health literacy is an important component of the approach of PM. Kickbusch and colleagues (2006) defined health literacy as 'the ability to make sound health decision(s) in the context of everyday life, - at home, in the community, at the workplace, the healthcare system, the market place and the political arena' [31]. Health literacy has gained increasing recognition by the European Institutions and is included in several of their policy documents [56]. The first European health literacy survey was conducted in 2011 as part of the European Health Literacy Project (HLS-EU) in order to measure the level of health literacy within eight Member States (MS) of the EU [56]. The results of the survey highlighted that the level of health literacy greatly differs among the participating countries. One key result of the survey was that people with a lower health literacy level are more likely to suffer from a lower health status compared to people with higher levels of health literacy $[5,56]$. Patient empowerment and health literacy are key components of the approach of PM.

\section{Solution - Good Governance via Health Data Cooperatives}

As potential solution to overcome the challenge of patient empowerment and health literacy across the EU we would like to propose the concept of health data cooperatives (HDC). In order to empower and literate patients to strengthen the uptake of PM, HDCs could be a democratic solution as it is suggested by Hafen and his colleagues [24]. HDCs not only promise to integrate big data in an effective, efficient, timely and socially acceptable way, it also promises to empower the patients/citizens by being part of and actively involved in the decision-making processes of the HDC. HDC will make patients/citizens proactive consumers of health also called 'prosumers'. Patients/citizens will be actively involved in 


\section{Chapter 8}

research ('citizen science') and be able to actively participate in the decisionmaking process regarding their health and treatment ('learning by doing'). It can be expected that empowered and literate patients/citizens will be the key in improving the diffusion of PM within the EU and its MS. Furthermore, in comparison to already existing health registers as for example rare disease registries, HDCs are owned and controlled by its members or in other words it is controlled by the citizens. By joining the HDC model, the citizen are not only in the driver's seat, citizens become citoyens. Since the economic value of personal data is immensely increasing and the world largest companies show increasingly interest in the collection of personal data and health data, the risk to suffer from misuse of the data by third parties, HDCs give the patient/citizen the responsibility for the storing, analyzing and sharing of their health data [24]. As a collective, society as such is the beneficiary of both the economic as well as the health value of the health related data and information. Furthermore, by each member having one vote, HDC members decide how the revenues generated by granting third parties access to their data that they agreed to share (respectively the data commons), should be invested (e.g., in research, in public health, in education, in community outreach etc.) [24].

\section{Challenge 2 - Integrating Big Data and ICT Solutions}

The development of PM will rely heavily on integrated 'big data' analytics and ICT solutions to generate the required knowledge and infrastructure to support the new approaches. Technologies for data capture and management and development of high quality databases will be instrumental, but there will also be a requirement for strategies to make sense of this big data for known and future purposes. Translational research infrastructures and data harmonisation of structured, semi-structured and unstructured data will be a central component of such strategies and should lead to new analytical methods and modelling approaches as well as innovative decision support tools such as in silico simulations to support physicians' decisions. To integrate all these aspects, further European big data and 'big science' frameworks need to be created and supported by suitable legislation. [47].

The world is challenged by a flood of information. In 2020 , there will be approximately 5.200 gigabytes of information of each individual across the globe [50]. The European Commission describes this flood of information as 'the big data paradigm' [11]. According to the European Commission 'a defining characteristic of today's data-rich society is the collection, storage, processing and analysis of immense amounts of data' [11]. The world's largest companies such as Apple, 
Microsoft and Google are more and more interested in the collection and storage of health data [23]. The economic value of personal information is steadily increasing in Europe and beyond [24, 51].

By applying 'big data' in healthcare and public health a new understanding of the complexity of diseases did evolve over the last year $[1,37]$. The analytics of big data make it possible to develop new medicines and drugs which are based on the individual molecular characteristics by integrating genomic information, lifestyle data and environmental information [37]. However, there is still room for improvements to make full use of the potential of big data because the majority of information is unstructured [29], inaccessible and stored in silos [24]. Since health and 'omics data are collected by an increasing variety of sources, the data collection is no longer seen as problem. The storage, analysis and integration of big data is currently challenging the professionals and healthcare systems traditional ways of working [1].

Big Data is commonly defined through its four V's: Volume, Velocity, Variety, Veracity [39]. According to a study by IDC [21, 28] the volume of data will double about every 2 years and will reach 40,000 Exabyte in 2020. This will be more than 5200 gigabytes per person in 2020.500 petabytes are currently generated in medicine only due to medical imaging and it is predicted that this number increases 50-fold until 2020 [42]. The 'omics revolution adds to the exponentially increasing data volume and given mobile technologies and sensors, the amount of data per person that can be captured in the future is expected to be in the order of 1100 terabytes during the person's lifetime [40]. Only $10 \%$ of this data will be clinical data, $30 \%$ are 'omics' data and the majority with $60 \%$ will be associated with exogenous data that captures lifestyle data, environmental data, behavioral data etc. The exponential increasing volume of data also indicates the speed that data is being generated, the second $V$ of big data. Just a simple example illustrates that data is generated in real time: Each patient in an intensive care unit generates continuous, real time data through all monitoring devices [41]. In our daily lives, mobile sensors are already capturing real time data continuously. The speed in which next generation sequencing can measure the human genome has increased drastically. Furthermore, continued progress in 'omics technologies' and new technological developments that allowed to drastically reduce the costs of the sequencing of the human genome [33]. Since 2001, the costs were cut from US \$ 100 million per human genome to around US \$ 1.000 in 2013 [55]. The increasing speed and lower costs now enable clinical routine use of the technology [60].

However, the other two V's of Big Data are currently posing the largest challenge. The Variety of medical data has a wide spectrum as indicated before ranging from doctor's letters, radiology reports, laboratory reports, 'omics data to mobile sensor data and even social media. To integrate and correlate all this data 


\section{Chapter 8}

with the published knowledge and guidelines as well as best practices and human expertise poses a very large challenge to gain meaningful insight from big data. The more we can integrate lifestyle data from wearables for example as well as social media data or environmental data, the 4 th $\mathrm{V}$, Veracity should be considered when carrying out the analysis, e. g. it should be asked how much a twitter feed or google search data can be trusted.

\section{A Solution - Creating Fuzziness and Making Big Data Analytics Actionable}

Considering the characteristics and value of Big Data in medicine, it's application is an essential step towards individualized medicine. Cognitive computing and computer tools in general become unreplaceable in how we treat patients especially in the context of rare disease. Mechanistic models with predictive power will soon be able to simulate clincal trials and predict the associated benefits for patient welfare and economy. Many other areas (e.g. the automobile and aviation industries) have already transformed towards a data driven mindset and rely on modeling techniques to improve quality, decrease costs, accelerate development and reduce risks. Often, undiagnosed patients and patients with rare disease suffer from lack of democratization of knowledge meaning that every doctor should have the wealth and expertise of the medical profession at their fingertips. Furthermore, an increased virtualisation of the drug development process - with virtual clinical trials as one of the key components as well as more personalised therapy and prevention strategies based on patient modelling - might, in our ageing societies, very well be the only alternative to increased rationing of health care provision [32]. At the same time, services and data bases like Orphanet, OMIM, FindZebra, Isabel Healthcare and the IBM Watson technology, to name but a few, will support the physicians in finding the correct diagnosis and serve as assistants to accelerate differential diagnostics in individuals where there is no choice but to look at PM to diagnose and treat the rare disease.

Furthermore, also in this context good governance frameworks such as health data cooperatives (HDCs) will not only improve health literacy and empower patients, the integration of big data into a single system will improve the drug development process for rare disease and consequently will improve access to treatments for RDs. 


\section{Challenge 3 - Translating Basic to Clinical Research and Beyond}

In order for PM to reach its anticipated impact on human health and wellbeing, translation of discoveries and communication across the continuum of research are required. A Europe-wide process to evaluate and validate biomarkers, together with longitudinal and in-depth studies to further characterise diseases and their progression would support ongoing efforts towards this integration and reclassifcation. The development of new clinical trial designs that are adapted to these new approaches and the integration of preclinical testing with innovative clinical trials may further improve the effectiveness of interventions. Collaborative pre-competitive and transdisciplinary research and cross-sector collaborations need to be promoted and supported by suitable funding mechanisms in order to truly bridge all steps of the PM research continuum [47].

The new understanding of the complexity of diseases and that individuals show unique molecular characteristics is challenging the ways of working regarding the design of clinical trials. Clinical trials have been seen as the gold standard for many years but the traditional design of clinical trials is not applicable for the era of personalised medicine [36]. The traditional approach of designing clinical trials ignores the complexity of diseases [32] and the importance of the integration of big data [8], even in cases when it is known that patients differ in their response rates to drugs. Developments in epigenomic and genomic studies have led to a new human diseases classification [38]. Since patients' pools are becoming smaller, current clinical trial designs with up to thousands of participants cannot be sustained. N-of-1-trials are often seen as new design for clinical trials in the era of PM [36,54]. The idea of $\mathrm{N}=1$ trials is that each individual/patient will be used as his/her own control/reference point. Since each patient will act as his/her own reference point, continuous data collection of health information over years or when it is possible lifelong is needed. Data collection is a dynamic and changing process and therefore it allows intraindividual follow ups and comparisons [36].

As already mentioned above, many of the common conditions we know will be broken down into small subsets of disease with small patient populations that may fit into the definition of rare diseases. This will be possible due to the better understanding of the molecular causes of disease, the development of new biomarkers - static and dynamic- to define the characteristics of each patient, the possibility to integrate different data sources from each individual patient, and other scientific and technological developments. Therefore, we will no longer speak of "cancer" but "triple negative breast cancer", or "PIK3CA mutated squamous cell lung cancer". Centuries ago infectious diseases were considered as 


\section{Chapter 8}

one big pot of diseases and nowadays we differentiate very clearly each of them with their differing pathogens and their dramatic differences, the same is already happening or will happen soon in oncology and many other diseases that are still clustered.

Rare or orphan diseases have been facing the challenge of small populations for all long time. They were challenged by clinical trials with low statistical power, the impossibility to gather enough evidence for marketing authorization applications, the indifference from drug developers due to the limited market and disseminated patients with difficulty to establish contact, between each others. In the cluster of rare diseases, all stakeholders joined forces and learned from each other to establish new pathways and overcome these challenges. Instead of reinventing the wheel, we should look at sources and methods developed in the field of rare diseases and apply them to PM, as for example rare disease registers and registries. Such registries make it possible to pool data to gather a sufficient sample size for epidemiological and clinical research even in times of smaller patient populations.

\section{A Solution - Making use of Registries}

Traditional dieases registries in the sense of 'common disease regisistries' such as cancer registries and rare disease registries have been used many years to collect data and information about diseases and rare diseases and their treatments across Europe. Furthermore, they have served as key tool to assess clinical outcomes and for the assessment of technologies. Rare disease registries are often built up on national or local level to map RDs in certain areas and to collect information regarding the incidence and prevalence of different RDs in those selected areas. Beside general rare disease registers which are holding information and data on many different RDs, registries which are focusing on one specific rare disease also exist in the EU. Data for those disease registries are mostly obtained on a voluntary basis, observational studies and clinical data.

Traditional disease registries and rare disease registers are important tools for making use of PM and further strengthen the implementation of PM in healthcare systems across the EU. For example, often patients that are suffering from rare conditions are lacking access to adequate care. Further, their obtained health data is collected and stored in silos and accordingly inaccessible or incompatible with other data sources.

Pancreatic cancer can be placed at the interface between rare and common diseases. Its incidence is increasing due to factors including demographic change. Pancreas cancer, (still) defined as a rare cancer, has the lowest survival rate of any cancer. Death rates from pancreas cancer are rising across Europe 
and beyond while those from all other cancers continue to fall. It is predicted that in 2030 pancreas cancer will be the second most frequent cancer. There is no option to control pancreas cancer incidence or mortality by primary or secondary (screening) prevention and only minor advances have been done recently in tertiary prevention under the umbrella of personalised treatment. Recently, the EC, identified pancreas cancer as a tracer in bridging "rare" and "common diseases".

As demonstrated for the case of pancreas cancer, harmonized registries including the traditional disease registries as well as rare disease registries will be major facilitators to understand the complexity of diseases, to conduct clinical trials, to improve the drug development process and to strengthen the uptake of PM across the EU.

\section{Challenge 4 - Bringing Innovation to the Market}

Bringing innovative PM solutions to the market presents a new set of challenges, including the issue of uncertainty. There will be opportunities to support the development of new risk-based approaches for the evaluation of PM in a context that encourages systematic early dialogue with all stakeholders, including regulators, funders and innovators, providing guidance for companies to enter the market for PM. As is the case for the research continuum, partnerships and innovation networks need to encourage cross-disciplinary and cross-border collaboration, and these would benefit from a transparent 'open Innovation'. Finally, research on appropriate policy, regulatory and legal frameworks would ensure that the new challenges associated with PM are adequately addressed from these perspectives [47].

In order to place an innovative product in a timely effective way on the market, the inherent uncertainties of innovation need to be considered. The implementation of an innovation to the market has traditionally been seen as a liner process "from research and development to regulatory approval, and then to health technology assessment (HTA) and on to the final reimbursement and implementation decision" [47].

However, the traditional market authorization processes, are not suitable for the approval of PM. The standard development process of drugs takes in average more than 10 years and costs up to a billion dollars [35]. The new understanding of the complexity of diseases makes it possible to design drugs that are more targeted to the patient's needs and therefore more effective than 'one-size fits all' drugs. 


\section{A Solution - Flexible Market Authorization Methods}

Since large phase III clinical trials are not feasible, requirements for marketing authorizations need to adapt to the characteristics of PM. Approaches such as the adaptive pathways pilot launched by the European Medicines Agency (EMA) in 2014 open the way to promising new flexible marketing authorization methods for PM drugs. Furthermore, the consortium working on 'Medicines Adaptive Pathways to Patients pilots (MAPPs)', lead by the EMA, are evaluating important open questions for the further development and application of flexible marketing authorization methods [35]. MAPPs 'refer to flexible development and access pathways within the current regulatory framework that balance early patient access, public health and societal benefits' [16].

Curently underlying outdated regulation is one of the main bottlenecks for the implementation of PM. Most of the relevant legislations and regulations such as for example the data protection regulation and medical devices regulation are currently under revision or have been recently revised. Hopefully the updated versions help to strengthen the uptake and implementation of PM $[8,9]$. However, this may be a potracted process, since the revision and regulation in Europe is often an extremely complicated and complex task [9].

In contrast, the clinical trial regulation, which was released in 2014 , is a perfect and rare example, how a regulation can be quickly revised. Improved and close collaboration and systematic early dialogue between all relevant stakeholders such as legislators, industry and other interest groups, made this possible [9].

Another regulation that is currently under revision is the data protection regulation [25]. During the time when the data protection regulation was published, it was not foreseeable how fast 'omics technologies' and the sequencing of the human will change the landscape of collecting, storing and analyzing personal data. To strengthen the uptake of PM, it is of great importance that the revised data protection regulation considers the increasing amount of available data and the different technologies by which the data is collected [9]. On the one hand the revision needs to take into account the protection of individuals and their personal information against misuse and stigmatization by third parties [26] and on the other hand research needs to be conducted without being hindered by overregulation. Moreover, harmonization of regulations and legislation is an essential step that is needed to strengthen the uptake of PM [36].

Furthermore, regarding the regulation of rare diseases, we should go one step beyond and asks ourselves if rare diseases should still be considered as a special group of diseases needing special pathways in the future? As we have seen, many conditions will divide into smaller clusters of disease and fit into the definition of rare diseases. Therefore, we will no longer need to develop special 
regulations for rare diseases. If we develop and apply them for the entire spectrum of new subconditions they will in any case be applicable for rare diseases.

\section{A Solution - Systematic Early Dialogue}

Systematic early dialogue (SED) is of great importance to bring the innovation in a timely effective way to the market. SED between the innovators, the end-users and the decision-makers ensures that innovators consider regulatory issues and reimbursement evaluation needs during the development process and will consequently lead to a more efficient innovation process [36]. SED between all stakeholders decreases the risk of duplication and misalignment of expectations and decreases the time to bring the innovation on the market. In conclusion, by applying SED, the risk that the innovation will end in the 'Death Valley of innovations' will decrease [35]. Furthermore, it brings the view of patients and HCPs into the review process and it provides guidance and clarity for the innovators throughout the whole innovation process.

\section{Challenge 5 - Shaping Sustainable Healthcare}

PM needs to rely on a knowledgeable healthcare system that is able to adapt to these new approaches in a timely and socially acceptable way, and that enables the participation of all stakeholders to increase PM's effectiveness and effciency. Patients and the citizen will play an increasingly important role in adopting and controlling the use of data from electronic health records and in developing prospective surveillance and monitoring systems for personal health data. To ensure the effectiveness of the healthcare system, health economics research relating to PM needs to be supported. In addition a exible framework for pricing and reimbursement equitable for all patients needs to be developed, leading to an overall healthcare nancing strategy that covers all aspects of PM [47].

Reimbursement questions and quality and data integration are important factors, which need to be considered to build and shape sustainable healthcare systems.

Not only is there a need to change the design of clinical trials, to revise outdated regulation and to find ICT solutions to better ingrate big data, there is also an urgent demand to adapt financing and reimbursement mechanisms across Europe and beyond [36]. The traditional reimbursement and pricing mechanisms which are currently in place are making the uptake and diffusion of PM often difficult. Moreover, it is argued by critics that PM will impose rather a higher economic burden for healthcare systems than making healthcare systems more 


\section{Chapter 8}

efficient due to the high costs of PM [58]. Since reimbursement systems across Europe are restrictive to pay for PM, EC Member States greatly vary in their ability to provide access to innovative therapies and medicines [17, 22, 30].

There is a clear lack of harmonization across the EU, due to the fact that the decisions with regard to pricing and reimbursement of pharmaceutical products and diagnostic tools are the responsibilities of the Member States and therefore made on a national or local level [22]. On the other hand, regulatory decisions are the responsibility of the EU [22]. Consequently, the EU is challenged 27 different pricing and reimbursement mechanisms and health technology assessments [34]. Even within countries different mechanisms exist such for example as it is the case in UK. To further strengthen the uptake of PM, harmonization is urgently needed regarding pricing and reimbursement tools across the EU, as proposed in the ENVI report on a harmonized EU assessment of the Added Therapeutic Value of Medicines [6].

\section{A Solution - Managed Entry Agreements}

One of the big question marks remains the issue of pricing and reimbursement. For this area, we still need to develop new methods beyond the ones existing for orphan drugs. The sustainability of healthcare systems will be challenged if the incentives and pricing strategies developed for orphan drugs are extrapolated to all PMs. New risk methods must be developed for pricing and reimbursement. The Managed Entry Agreements negotiated between individual EU countries and pharmaceutical companies offer good examples of possible risk-sharing mechanisms. However, their results should be evaluated and they must become transparent before they can be implemented on a larger scale [19].

\section{A Solution - European Reference Networks}

The European Commission established for rare diseases so called 'European reference networks' (ERNs) to integrate information on rare disease into one single system across the EU. According to the European Commission those ERNs "should serve as research and knowledge centres, updating and contributing to the latest scientific findings, treating patients from other Member States and ensuring the availability of subsequent treatment facilities where necessary. The definition of ERN should also reflect the need for services and expertise to be distributed across the EU" [10]. The Directive 2011/24/EU on the application of patients' rights in cross-border healthcare sets the rules for patients right to access safe and good quality treatment across the European borders and 
reimbursement roles. The directive provides a firm basis for increased cooperation between national health authorities. Some provisions address rare diseases. Article 12 foresees enhances cooperation of Member States including the criteria and conditions for ERNs for healthcare providers. The directive aims to identify already established centres of expertise and to encourage voluntary participation of healthcare providers in the future of ERNs.

ERNs can be seen as pilots to integrate information on rare diseases into one single system. If we will manage to integrate information on RDs on a European wide level into a single system by using ERNs, we will be able to harmonize data integration for other sectors as well. Improving data integration will greatly improve the drug development process and consequently the access to drugs for rare conditions.

\section{Conclusions}

Emerging technologies such as Whole Genome Sequencing (WGS), Whole Exome Sequencing (WES) or Low-Coverage Sequencing (LCS) have proven that recent failures in stratified medicine show the need for better understanding of the molecular basis of rare diseases (RDs). The continuing advances in scientific knowledge will facilitate the move from the current stratified approach, which relies on static biomarkers of a RD, to a truly individualized treatment, which considers the combination of dynamic biomarkers, dynamic risk profiles, RD heterogeneity in time and space, the ever changing environment, epigenomics and many other factors that modulate RD phenotype and response to treatment. For example, big data analytics (e.g. IBM Watson) has been identified as a tool for the management of RDs and solving the challenges in the monitoring of a RD of an individual patient over time and space, i.e. taking into account the dynamics of individual patient information.

Conclusions can be drawn, that, on the one hand, the field of RDs has stimulated and pushed discussions and solutions in other fields. On the other hand, the final 5 challenges for personalised medicine, which had been identified by the PerMed SRIA, apply to all diseases including RDs. Since the vision of PM implies that the idea of common diseases will be replaced by unique disease profiles, there are no specific research and policy needs for RDs. This result has enormous implications for European and national policymakers. Instead of asking for separate regulations for RDs, it asks for future regulations and infrastructures (e.g. ERNs), which apply to all diseases in the same way. Rare cancers had been identified as a best practice example and role model to prepare and guide EU Member States in that direction. 


\section{Acknowledgment}

Part of the research leading to this book chapter has received funding from the European Union Seventh Framework Programme (FP7/2007-2013) under grant agreement No. 602139 (CSA PerMed, 2013-2015) and under grant agreement No. 305690 (RARE-Bestpractices, 2013-2016). 


\section{References}

1 Alyass A, Turcotte M, Meyre D (2015) From big data analysis to personalized medicine for all: challenges and opportunities. BMC Med Genet 8(1):33

2 Auffray C, Balling R, Barroso I, Bencze L, Benson M, Bergeron J, Bernal-Delgado E, Blomberg N, Bock C, Conesa A (2016) Making sense of big data in health research: towards an EU action plan. Genome Med 8(1):1

3 Bereczki D (2012) Personalized medicine: a competitor or an upgrade of evidence-based medicine? Per Med 9(2):211-221

4 Boycott KM, Vanstone MR, Bulman DE, MacKenzie AE (2013) Rare-disease genetics in the era of next-generation sequencing: discovery to translation. Nat Rev Genet 14(10):681-691

5 Cutica I, Mc Vie G, Pravettoni G (2014) Personalised medicine: the cognitive side of patients. Eur J Intern Med 25(8):685-688Directorate-General for Internal Policies - European Parliament (2015) Towards a harmonised EU assessment of the added therapeutic value of medicines. European Parliament, Brussels

6 Estape EA, Mays MH, Sternke EA (2016) Translation in data mining to advance Personalized medicine for health equity. Intell Inf Manag 8(01):9

7 European Alliance for Personalised Medicine (2013) Innovation and patient access to personalised medicine. Report from Irish Presidency Conference Brussels

8 European Alliance for Personalised Medicine (2014) MEP's Briefing Paper 2014-2019 Legislature

9 European Commission (2016) European networks of reference for rare diseases. http:// ec.europa.eu/health/rare_diseases/european_reference_networks/erf_en. Accessed 7 Dec 2016

10 European Commission (2014) The use of big data in public health policy research

11 European Commission (2016) Towards an International Consortium for Personalised Medicine (IC PerMed)

12 European Commission (2012) Biobanks for Europe. A challenge for governance Belgium

13 European Commission (2013) Use of "-omics" technologies in the development of personalised medicine Belgium

14 European Commission (2014) Advice for 2016/2017 of the Horizon 2020 advisory group for societal challenge 1, "Health, Demographic Change and Wellbeing"

15 European Federation of Pharmaceutical Industries and Associations (2014) What are Medicines Adaptive Pathways to Patients (MAPPs)

16 European Federation of Pharmaceutical Industries and Associations (2010) Patients W.A.I.T. Indicator

17 European Science Foundation (2012) Personalised medicine for the European citizen. Towards more precise medicine for the diagnosis, treatment and prevention of disease (iPM) European Science Foundation, Strasbourg November

18 Ferrario A, Kanavos P (2013) Managed entry agreements for pharmaceuticals: the European experience. London school of economics and political science 154

19 Francioso S, Almerighi C, Forte P, Bandiera F, Nosotti L, Lionetti R, Taliani G, Piras MR, Ponti ML, Parruti G (2014) A simple rule to personalize standard dual therapy across all genotypes in naive chronic hepatitis C patients: the TT4 randomized trial. Dig Liver Dis 46(2):164-169

20 Gantz J, Reinsel D (2012) The digital universe in 2020: big data, bigger digital shadows, and biggest growth in the far east. IDC iView: IDC Analyze the future 2007:1-16

21 Garfield S (2011) Advancing access to personalized medicine: a comparative assessment of European reimbursement systems. Personalized Medicine Coalition Bridgehead International

22 Gomes L (2015) Tech giants bet on biometrics. IEEE Spectr 52(6):52-55

23 Hafen E, Kossmann D, Brand A (2014) Health data cooperatives-citizen empowerment. Methods Inf Med 53(8) 


\section{Chapter 8}

24 Horgan D, Jansen M, Leyens L, Lal JA, Sudbrak R, Hackenitz E, Bußhoff U, Ballensiefen W, Brand A (2014) An index of barriers for the implementation of personalised medicine and pharmacogenomics in Europe. Public Health Genomics 17(5-6):287-298

25 Horgan D, Paradiso A, McVie G, Banks I, Van der Wal T, Brand A, Lawler M (2015) Is precision medicine the route to a healthy world? Lancet 386(9991):336-337

26 lanuale C, Leoncini E, Mazzucco W, Marzuillo C, Villari P, Ricciardi W, Boccia S (2014) Public health genomics education in post-graduate schools of hygiene and preventive medicine: a cross-sectional survey. BMC Med Educ 14(1):213

27 IDC (2016) Big data and analytics is increasingly a game of inches. https://www.idc.com/ prodserv/4Pillars/bigdata

28 Intel (2013) Care customization: Appying big data to clinical analytics and life sciences

29 Kanavos P, Vandoros S, Irwin R, Nicod E, Casson M (2011) Differences in costs of and access to pharmaceutical products in the EU

30 Kickbusch I, Wait S, Maag D, Banks I (2006) Navigating health. The role of health literacy. Alliance for Health and the Future, International Longevity Centre-UK, London

31 Lehrach H (2015) Virtual clinical trials, an essential step in increasing the effectiveness of the drug development process. Public Health Genomics 18(6):366-371

32 Lehrach H (2012) A revolution in healthcare: challenges and opportunities for personalized medicine. Per Med 9(2):105-108

33 Leopold C, Vogler S, Habl C, Mantel-Teeuwisse A, Espin J (2013) Personalised medicine as a challenge for public pricing and reimbursement authorities-a survey among 27 European countries on the example of trastuzumab. Health Policy 113(3):313-322

34 Leyens L, Brand A (2016) Early patient access to medicines: health technology assessment bodies need to catch up with new marketing authorization methods. Public Health Genomics 19(3):187-191

35 Leyens L, Horgan D, Lal JA, Steinhausen K, Satyamoorthy K, Brand A (2014) Working towards personalization in medicine: main obstacles to reaching this vision from today's perspective. Per Med 11(7):641-649

36 Leyens L, Reumann M, Malats N, Brand A (2017) Use of big data for drug development and for public and personal health and care. Genet Epidemiol 41(1):51-60

37 Loscalzo J, Kohane I, Barabasi AL (2007) Human disease classification in the postgenomic era: a complex systems approach to human pathobiology. Mol Syst Biol 3(1):124

38 Martin-Sanchez F, Verspoor K (2014) Big data in medicine is driving big changes. Yearb Med Inform 9(1):14-20

39 McGovern L, Miller G, Hughes-Cromwick P (2014) The relative contribution of multiple determinants to health outcomes. Health Aff (Millwood) (2):1-9. http://healthaffairs.org/healthpolicybriefs/brief_pdfs/ healthpolicybrief_123.pdf

40 McGregor C, James A, Eklund M, Sow DM, Ebling M, Blount M (2013) Real-time multidi- mensional temporal analysis of complex high volume physiological data streams in the neonatal intensive care unit. In: Medlnfo, 362-366

41 Meyer M (2015) Digitale chancen: next generation healthcare - siemens keynote

42 Negrouk A, Horgan D, Gorini A, Cutica I, Leyens L, Schee genannt Halfmann S, Pravettoni G (2015) Clinical trials, data protection and patient empowerment in the era of the new EU regulations. Public Health Genomics 18(6):386-395

43 Ogino S, Fuchs CS, Giovannucci E (2012) How many molecular subtypes? implications of the unique tumor principle in personalized medicine. Expert Rev Med Diagn 12(6):621-62

44 Palau F (2012) Personalized medicine in rare diseases. Per Med 9(2):137-141

45 Pavelić K, Martinović T, Pavelić SK (2015) Do we understand the personalized medicine paradigm? EMBO Rep 16(2):133-136

46 PerMed (2015) Shaping Europe's vision for personalised medicine - Strategic Research Innovation Agenda (SRIA) 
47 Powers Dirette D (2015) Personalized medicine: definitions, history, and implications for the OT profession. Open JOccup Ther 3(4):1

48 Roden DM (2015) Cardiovascular pharmacogenomics: current status and future directions. J Hum Genet 61(1):79-85

49 Rometty V (2014) The year of the smarter enterprise. The economist, The world in

50 Rose J, Rehse O, Röber B (2012) The value of our digital identity. Boston Cons Gr

51 Sackett DL, Rosenberg WM, Gray JM, Haynes RB, Richardson WS (1996) Evidence based medicine: what it is and what it isn't. BMJ 312(7023):71-72

52 Schleidgen S, Klingler C, Bertram T, Rogowski WH, Marckmann G (2013) What is personalized medicine: sharpening a vague term based on a systematic literature review. BMC Med Ethics 14(1):55

53 Schork NJ (2015) Personalized medicine: time for one-person trials. Nature 520(7549):609-611

54 Snyderman R (2014) Personalized medicine 2014: has healthcare been transformed? Per Med 11(4):365-368

55 Sørensen K, Pelikan JM, Röthlin F, Ganahl K, Slonska Z, Doyle G, Fullam J, Kondilis B, Agrafiotis $D$, Uiters $E$ (2015) Health literacy in Europe: comparative results of the European health literacy survey (HLS-EU). Eur J Public Health 25(6):1053-1058

56 Spear BB, Heath-Chiozzi M, Huff J (2001) Clinical application of pharmacogenetics. Trends Mol Med 7(5):201-204

57 Taylor D, Al-Saeed E (2010) Can (and will) governments afford personalized medicine? Per Med 7(5):587-595

58 Turner RM, Pirmohamed M (2014) Cardiovascular pharmacogenomics: expectations and practical benefits. Clin Pharmacol Ther 95(3):281-293

59 Zhang J, Chiodini R, Badr A, Zhang G (2011) The impact of next-generation sequencing on genomics. J Genet Genomics 38(3):95-109 

Discussion \& Future perspectives 

The implementation of PM is a major objective in Europe and elsewhere. Substantial research has been conducted to support the idea and overcome challenges that its realization still face. The aim of the dissertation was to illustrate translational aspects in order to move from genetic focus towards more holistic systems thinking in PM. Based on the definition of the European Commission Horizon 2020 Advisory Group for Societal Challenge "Health, Demographic Change and Wellbeing" and drawing on the PerMed 'Strategic Research and Innovation Agenda (SRIA)' five selected dimensions of PM have been addressed in this dissertation including personal genomics and genetic data sharing, the integration of Big Data for policy empowerment, the case for chronic complex disease and lifestyle, the introduction of mHealth innovation in the market and the overall contributions of these dimensions to shaping sustainable healthcare systems. In the following, the main findings have been summarized and discussed in the terms of their contribution to answering the main research question. The discussion has been organized according to the five challenges and the previously defined research objectives. In each section remaining questions have been discussed. A critical analysis of limitations of each part, as well as an overall estimation of valorization of this dissertation will follow.

\section{Personal Genomics as a mean to participate in science}

Overall, medicine became and will increasingly be a data intensive enterprise. The growth of research activities to collect human bio samples to better understand health and disease is spectacular and the nature of data is constantly evolving [122]. In this context the first objective was to explore the societal perception of personal genomics and genetic research participation, the value people attribute to genetic research and the types of trade-offs between privacy and utility that people are willing to accept.

The findings of the first publication [82] (Chapter 1) indicated a relatively positive overall attitude towards personal genomic testing among older Swiss adults. One third of the participants were aware of personal genomics and the majority expressed an interest in undergoing testing. In general, participants were motivated to learn about their personal disease risk and by the opportunity to contribute to scientific research. Their motivation to share data was mainly encouraged by altruistic reasons, based on their interest in contributing to the greater good for next generations without any expectation of reciprocity. Donating data for research was even perceived as a personal utility. Furthermore, several factors were highlighted that might impact one's willingness to share data such the access and utilization of this data by private companies, generational differences in the attitude towards sharing data, differences between sharing genetic data or 
health data and the presence of financial incentives. In addition, the study found that participants trusted academic research institutions, and that the older adults surveyed accepted the compromise between supporting scientific advancement while risking the protection of privacy. Some participants expressed concerns regarding the questionable nature and validity of the results. However, only a minority of respondents mentioned privacy-related reservations. Lastly, some participants indicated worries regarding data sharing such as the misuse of data, the fear to become a transparent citizen and data safety.

In sum, the value that participants of the studies attribute to genetic research mainly lies in the advancements of science for societal development, their personal contribution to research as well as by the chance to give back to society. This altruistic motivation influences their trade-off between privacy and utility. While privacy, confidentiality and fear of discrimination are usually cause for debate, in this study, it was concluded that the risk of diminished assurance of privacy due to data disclosure has been outweighed by the high potential benefit of research participation. However, transparency, data protection and trust in research institutions remain prerequisites of this openness. The patterns of results suggest, that there is a high potential for citizen science in academic research. This integration of patients into the conduction of research and clinical studies, as well as the definition of initial research questions is very valuable. Specifically in the group of older adults, who are typically excluded from research, are a very important source of information. First, due to the changing demographic distribution, engagement of older patients in managing their own health and disease is highly important. Second, the combination of disease history and genetic profiles may lead to in-depth insights on longevity and the development of disease. Furthermore, this group is less concerned by the risk to give up privacy. Furthermore, patient-reported outcomes of treatments, evaluation of medical interventions and post-marketing surveillance of medicines could be enclosed in academic citizen science research agendas [121].

However, some questions remain: First, giving data voluntarily to support the greater good without any return is a phenomenon that has to be protected. In citizen science, lots of interests are at play and people are at risk of getting exploited [122]. Therefore, people need credit for their openness to ensure fairness. Second, science is a closed system and findings remain within the scientific community, despite the fact that the Universal Declaration of Human rights states in Article 27: "Everyone has the right freely to participate in the cultural life of the community, to enjoy the arts and to share the scientific advancements and its benefits" [123]. Therefore, future investigations should explore the following questions:

Open Question (1): How can the willingness of people to share their data be protected and how can exploitation be prevented? 
Open Question (2): How "fair" is sharing data for all stakeholders and which benefits could be returned to the data subject?

Third, in the specific case of genetic data, there is still much discussion regarding whether the general population is literate enough to decide over their genetic data, able to make serious decisions based on their genetic data and whether people are able to understand the predictive nature of genetic results. The lack of genetic literacy is a prominent issue. In many cases, misconceptions of the value and limitations of genetic data have been observed [124].

Open Question (3): How to inform and educate people appropriately to overcome the lack of genetic literacy, give people the feeling of being in control and respect their autonomy without creating a burden?

\section{Health Data Cooperatives}

Overall, the increase in data leads to new insights and allows for emerging types of data and new content, which enables to answer questions that were previously considered beyond reach. The true value of data lies in the combination and integration of different data sets. Not only stratification but also the integration of dynamic data sets will result in insights on real disease heterogeneity, the impact of changing environments and epigenetics. This is of great value in the case of PM. Therefore, the second objective was to investigate the potential of Big Data as a support tool for making innovative decisions in public health policy making. Chapter 2 [94] discussed the value of Big Data and need for accessing and sharing of data to realize its full potential. Within the article, we suggest the implementation of Health Data Cooperatives (HDC) as a key element for empowering policy makers and to overcome current public health policy challenges. An HDC is a bottom-up trusted ecosystem that integrates stakeholders' data, knowledge, materials and services into a cloud-based framework. The diversity of health data sources integrated in the HDC is processed, analyzed and mapped comprehensively in order to create a network of knowledge and actionable insights. Furthermore, HDCs would be designed to overcome the multidimensional challenges of the highly heterogeneous data landscape in health care, as well as data ownership and control. On this basis, public health policy makers would have accurate data access and therefore be empowered to monitor and evaluate policy changes and make future data-driven decisions. All of this might result in significant strategic, societal, economic and political advantages.

To reach these goals and integrate HDC in the proposed manner, the ambitious concept of a European-wide HDC broken down into smaller pieces for testing and step-wise implementation might lead to the intended goal. A potential first 
step may be the development and implementation of the electronic health record. Some countries are already successful on a hospital level. However, for extrapolating the idea on an EU level, more testing scenarios need to be evaluated; more forms and purposes need to be investigated in more detail, for a successful application on a European level. Therefore, one of the remaining challenges will be:

Open Question (4): How to coordinate cross-border networking and integrate technology that will facilitate interoperability among the components of Big Data value chain and therefore enable the realization of HDC?

Second, international data sharing principles and policies need to be developed to enable cross-sectoral and transnational data sharing. Even though we propose, that legal protection will be integrated into the HDC model, there is still a need for overarching data governance principles to find a balance between ethical risks and scientific progress. Ownership issues, data management, data quality and standardization need to be clarified in legislation. In this context, the current approach of consent needs to be reconsidered. Therefore, the focus of data governance principles should be the data subjects, or those to whom the data pertains, in addition to various other stakeholders. A remaining question would be:

Open Question (5): How to create a data governance principle that positions the data subject in an active role with direct control of and responsibility for their data, and that exhibits maximal respect and an optimal transfer of values and interests?

\section{Influence of Lifestyle on Chronic Complex IBD}

Chronic complex diseases with multiple pathogenic components cannot properly be controlled and much less cured by modulating single components at sporadic time points in the course of the disease or administering the same treatment to all patients. Further, more emphasis should be placed on disease prevention. Lifestyle interventions have been tested as a promising supporting treatment approach in a large variety of cases involving chronic inflammatory diseases, resulting in reduced inflammation and improvements to quality of sleep, mental wellbeing, fitness and daily physical activity. Since current approaches to treatment remain ineffective, the fourth objective of this dissertation was to explore the influence of a lifestyle intervention, including regular physical activity, on inflammation, fitness, depression and sleep in pediatric patients with IBD.

In summary, the four articles of Part 3 [102-105] show the following: At baseline, participants with IBD in an active state of the disease (IBD-AD) showed 
higher markers of inflammation including erythrocyte sedimentation rate (ESR), C-reactive protein (CRP) values, haemoglobin, and leukocyte values. IBD-AD had poorer psychological functioning and lower PA (average steps per day) compared to participants with IBD in remission (IBD-RE) and healthy controls (HC). No mean differences were found between IBD-RE and HC. In the second article, sleep behavior was investigated in more detail. Compared to HC and IBD-RE, IBD-AD patients showed impaired objective sleep patterns (e.g. more awakenings, longer sleep latency, and reduced stage 3 sleep). Linear relationships described the correlation between higher ESR and more stage 4 (minutes, percentage) sleep. Nonlinear relationships described the relation between ESR and subjective sleep quality (inverse $U$-shaped) and between CRP and sleep latency (Ushaped). In the third article, effects of the 8-week physical activity intervention were evaluated. Based on the data of this study we found improved exercise capacity of all participants. Self-reported fitness and daily physical activity increased in IBD-AD, but not in IBD-RE and HC. No improvements were observed for psychological functioning, depressive symptoms or subjective sleep. Objective sleep improved, deep sleep increased, light sleep decreased, and in the IBD-AD group the number of arousals after sleep onset also decreased. Descriptively, the IBD$A D$ group reported lower psychological functioning and poorer subjective sleep quality. Finally, after a single bout of exercise inflammation markers (albumin, haemoglobin, erythorcytes, haematocrit, leukocytes) strongly increased in all three groups. However, after a long-term AET intervention, inflammation markers ESR, CRP and thrombocytes significantly decreased.

As supported by these findings, the role of lifestyle factors in IBD is crucial, and may affect treatment outcomes. The studies show that regular physical activity results in a reduction in inflammation, improvement in fitness and improvement in mental well-being. Therefore, it has been suggested that moderate PA may be recommended as an adjuvant therapy in pediatric IBD for its various beneficial effects. This would contribute the improvement of existing treatments and potential new treatment options through retrospective evaluation.

However, although these studies assessed a large variety of outcomes, interactions of the individual disease circumstances with respect to genes was absent. Since the value of genomic data is increasingly understood, an integration of such data into clinical trials is necessary. Fortunately, this observation was shared by Andersen et al. [95] in their study proposal: "Treatment selection and lifestyle recommendation in chronic inflammatory diseases: A Danish multidisciplinary collaboration on Prognostic Factors and Personalized Medicine". The study aims to identify molecular markers for individualized treatment targets, phenotypic characteristics, as well as lifestyle factors that show a beneficial effect in treatment outcomes. This is in line with the innovative approach of Personalized Lifestyle Medicine, introduced by Minich and Bland (2013) [125]. Personalized 
Lifestyle Medicine is "an approach to medicine in which an individual's health metrics from point-of-care diagnostics are used to develop lifestyle medicine oriented therapeutic strategies for improving individual health outcomes in managing chronic disease" [125]. In practice, this may imply the identification of genetic and molecular markers to define patient-specific interventions for nutrition, exercise, stress and environment. Based on this innovative paradigm, in the future the following questions should receive attention:

Open Question (6): How to create appropriate algorithms and statistical methods that enable extraction of clinically useful information out of the multiomics information to capture an individual's response to lifestyle interventions?

Open Question (7): What kind of professional training or data visualization solution is necessary to optimally translate complex omics information into suitable information for patients' own understanding and support them in changing their behavior?

\section{MoVit as mHealth Innovation}

Adherence and lifestyle represent crucial aspects in potential treatment success to improve the patients' health and decrease costs [127]. With the improvements in and greater prevalence of wearable electronics, miniaturized sensor technologies, and mobile phone penetration, novel opportunities for personalized lifestyle interventions are provided to potentially support health care practice. With this in mind, the fifth objective was to create a tool that will translate scientific evidence on behavior change into a personalized program to support general practitioners (GPs) and empower patients to start a physically active life.

The business plan "MoVit" illustrates the dynamic nature of constant development in terms of scientific and technical advances and the integration of external factors such as lifestyle and environment in PM. MHealth is one aspect in this innovative environment and serves as a promising tool for integrating external factors, including lifestyle, into the health care context. The online application "MoVit", aims to translate scientific evidence of behavior change into a personalized physical activity coaching program and therefore, a support tool that would enable health care professionals to promote an active lifestyle among their patients. As applied in MoVit, the concept of gamification is increasingly designed to not simply kill time and create fun, but also have a positive impact on health [127]. The combination of pleasure, entertainment and evidence-based behavior change theories can motivate the patient to make informed decisions on daily activities that contribute to one's health. One concrete example in this context is 
the Pokémon Go app, which led people of all ages (mainly young people) increase their steps per day significantly [128]. Furthermore, mHealth application beyond lifestyle, such as medical diagnostic procedures might soon be integrated in medical care. This way they could be performed at home using portable medical mobile applications with specific patient customization. In line, telemedicine or robot support for remote health care services have the potential to allow more flexible doctor-patient connectivity across previously impossible distances [129]. Additionally, regulators will benefit from this close monitoring of patient-related outcomes. However, despite these promising tools the following questions remain:

Open Question (8): How to further minimize the burden of data collection and data interpretation for the patient as well as the health care professional?

Open Question (9): How to develop sustainable business models that enable research strategies for disease prevention innovation?

\section{Shaping Sustainable Health Care}

On one side, PM holds great promise for health care systems to become more effective in providing more tailored treatments for the individual patient. On the other side, the realization of such an innovative approach like PM is highly dependent on a knowledgeable system that is able to adapt to these new approaches and facilitate involvement of all stakeholders [38]. In this context, the fifth objective was to discuss the role of health care systems and policy making when shifting focus from traditional disease categorization towards unique disease profiles. Chapter 8 [119] discussed the impact of new disease categorization based on new emerging data sources, such as genetics. In this article it has been argued, that the idea of common disease will be replaced by unique disease profiles. Next to a new understanding of disease in the medical field, this result may have enormous implications for European and national policy makers. In the article, the case of rare diseases (RD) has been used to illustrate the scenario and leads to the statement that, instead of asking for separate regulations for RDs, it asks for future regulations and infrastructures, which apply to all diseases in the same way. The contemporary differences in regulation between RDs and common diseases should be equalized. Rare cancers had been identified as a best practice example and role model to prepare and guide EU Member States in that direction. It was concluded that all diseases are rare, every patient unique and trials be design with $\mathrm{N}=1$. 
As a crucial enabler in this process a system, the HDC has been proposed earlier. Next to the integration of data, it may convey multiple stakeholder perspectives into the process and will enable a user-centered design process throughout the whole innovation progression. To guarantee sustainability, bottom-up population support and acceptance is essential. Therefore, regulations on informed consent and data protection legislation are major priorities. As soon as this foundation is assured, electronic patient records, registries and biobanks can further develop towards HDCs and leverage practical benefits for patients. In addition, insights on disease prevention and treatment, as well as lifestyle recommendations create opportunities to improve public health and reducing health care cost for more sustainable health care systems. To facilitate this development, early dialogue among all stakeholders, as well as thorough coordination of labors will decrease the risk of duplication of efforts and misalignment of expectations [80]. To further raise awareness among all sectors, another question is:

Open Question (10): How to promote engagement and close collaboration between all stakeholders across science, sectors and borders through HDC?

In parallel, next to continuously investing into research and innovation, the sustainability of healthcare systems will be highly dependent by the choice of future pricing structures. The unsustainable nature of todays' health care management and health care systems reimbursement structure has frequently been criticized. For the scenario of highly individualized treatments, simply extrapolating incentives and pricing strategies developed for orphan drugs to all PMs would not serve the goal of sustainability. Indeed, both approaches lack strong evidence to proof their benefit and governments are concerned about their cost-effectiveness and budget impact as compared to other health care interventions [130]. Therefore, the considerations of "quality evidence" in payer guidelines are needed, as well as strategies to incentivize socially relevant development [67]. A detailed elaboration on this would go beyond the scope of this work, however, two options that aim to incentivize high quality care, efficiency and flexibility, will be shortly introduced: First, the approach of outcome-based payment methods have been discussed in the context of PM. Outcome or value based pricing strategies are established on the estimated value of a product or service and has the potential to increase healthcare quality and by that promote long-term dynamic efficiency among health care providers and innovators [131]. There is a growing consensus that the current fee for service system of health care does not succeed to fulfill health cares' mission of rewarding healing of a patient [130]. Outcome-based payment may support investments into more individualized treatment strategies as well as preventive methods with appropriate incentives [132]. Second, a potential method might be the innovative management approach: Lean. The core idea of lean is to maximize value for the customer/patient with fewer resources. 
This operating philosophy originates from Toyota Motor Company and facilitated constant development and perfection of assembly-line manufacturing, waste reduction and inventory management [133]. The approach encourages the development of a culture of continuous improvement, engages all members of the team in identifying issues and solving problems along the way, aims to reduce waste and to continuously improve the process. Therefore, lean management aims to enhance quality, capacity and safety while reducing costs [134-136].

Overall, further studies are necessary to compare the implications of different pricing approaches that embrace the social and personal value of treatment, are flexible to inter-individual differences and contextual needs, offer transparent monitoring systems and use resources efficiently for sustainable health care. Therefore, the following question remains:

Open Question (11): How to design an optimized overall health care financing strategy?

\section{Limitations}

Despite the novelty of the studies and their contributing in advancing the field, various limitations warrant over generalization and findings should be interpreted cautiously.

For the project discussed in Part 1, research participants were recruited as a large convenience sample via the Seniorenuniversität Zurich, a continuing education program for people above 65 years of age. Theses seniors represent a selected portion of the elderly population, since they have an above average level of education and are socially, physically and culturally active. Furthermore, due to the limited amount of participants that actually have genetic test experience and since no genetic tests were executed as part of the study, survey questions were posed in a hypothetical manner. As the respondents were aware of the hypothetical nature of the questions, answers might have been influenced and differ from those in a real-world scenario.

The opinion articles in Part 2 and 5 aim to report the authors' perception and innovative vision of the current field of PM. Ultimately the authors aim to increase cross-disciplinary, cross-sectorial and cross-border attention for the topic of holistic PM supported by HDC. The main limitation of these articles is that they do not contain unpublished or original data.

The four articles of Part 3 all discuss findings that have been obtained within a larger project (IBD-Study) and have been published with different areas of focus. The strength of the project is that it appears to be the first in-depth investigation of children and adolescents with IBD. In detail, the study is unique in assessing the effect of a long-term physical activity intervention among a pediatric 
sample of patients with IBD. However, limitations of the studies were present: First, the relatively small sample size, especially in the group of children with an active state of the disease led to difficulties in discovering significant findings. To overcome this issue, effect sizes which are insensitive to small sample sizes have been applied. Second, during the recruitment of patients the focus has been on the German speaking part of Switzerland and included all patients willing and able to participate without further restrictions. Third, even though we tried to collect a wide variety of outcomes, a potential confounding effect of external factors, e.g. environmental influences or influences of the microbiome could not be eliminated. Fourth, the studies did not distinguish between different subcategories of the disease, but split groups according to disease severity. Fifth, for the intervention study, no non-intervention control group was included, which limited the ability of the study to detect intervention effects. Sixth, the intervention period, the intensity and frequency of the intervention or the baseline health of children may have hindered the ability to detect significant improvements. Seventh, the potential of treatment effects cannot be exclude, since participants were aware of the study condition.

The business plan in Part 4 has not been considered in this section, since scientific assessment criteria to estimate limitations of a business plan are not appropriate.

\section{Conclusions}

Despite various calls for a more holistic view of PM, this term remains a proxy for the application of genetics to individualized therapies. However, the continuing developments in technology and scientific exploration will facilitate the move from a traditional or stratified approach based on static biomarkers, to a truly individualized treatment that considers the combination of dynamic biomarkers, dynamic risk profiles, disease heterogeneity in time and space, the ever changing environment, epigenomics, lifestyle choices and many other factors that modulate the disease phenotype and response to treatment. This dissertation sheds light on a multidisciplinary, multidimensional and partially visionary approach on how to overcome the PerMed SRIA challenges to further facilitate this evolution of holistic PM. While the main research question is discussed on a meta-level, several actionable projects have been extracted and provide scientific evidence to the concept of holistic PM, as well as triggering new lines of thought.

First, to create awareness and empowerment among citizens and patients, an increased focus on general public engagement, especially of older adults, in scientific research activities known as "citizen science", initiated by public or academic research institutions may further strengthen the uptake of PM. "Citizen 
science" has the potential to lead towards an improved dialogue between science and society, as well as the integration of society into the process of co-creating scientific knowledge.

Second, to integrate Big Data and ICT Solutions, the incorporation of multiple data sets on a cross boarder level is essential. A Pan-European data sharing Platform or (Health) Data Cooperatives (HDC) has been proposed as an essential tool to tap into the potential of all existing and rapidly emerging data sources. Such a HDC would present new leverage to help overcome computational, algorithmic and technological challenges in data mining that characterize today's highly heterogeneous data landscape. Furthermore, it could serve as a host of diverse regulatory, normative, governance and policy constraints. HDC and the integration of multiple sources of data on an international level will lead to new insights and increased effectiveness on multiple levels.

Third, to translate basic to clinical research and beyond, more emphasis should be placed on prevention. Lifestyle and behavior change interventions have demonstrated to be successful strategies to increase treatment effectiveness, enable prevention and reduce costs. The increased efficacy through personalization will lead to patient-empowerment, the feeling of self-efficacy and the return of control for chronically ill patients. The next step is therefore, to improve health outcomes based on individual health metrics and personal preferences and integrating genetics and molecular markers for improved targetization.

Fourth, aiming to bridge innovation to the market, the MoVit mHealth application was presented as a concrete example of how to empower patients and health care professionals to put evidence-based lifestyle recommendations into clinical practice. MHealth again, produces data that might improve patient empowerment, self-efficacy, and health literacy and may contribute to informed decision making in the health care context. The integration of mHealth data into the overall health data pool would lead to new insights and enable alternative treatment strategies. To realize this goal, again, HDC might serve as a solution.

Fifth, to shape sustainability, health care systems require to remain flexible and dynamic, to incorporate the evolving role of patients, to integrate multiple stakeholders and to develop new frameworks of pricing. For PMs' realization, a significant paradigm shift will need to take place to fully embrace the new approach.

Considering today's rate of development, the current discussion on expanding the perspective of PM towards a holistic systems approach with the goal of improving prevention, prediction, personalization and participation for all stakeholders of the health care system might remain an ever evolving model of transformation. Technology will be the main driver in this fast paced development. However, patient-centeredness should be the ultimate goal of personalization. Therefore, the application of PM should remain flexible and dynamic so as to 
incorporate the evolving role of value systems and morals of patients, to integrate exponentially developing technology and to be prepared for what is yet to come: Lean Personalized Medicine? 


\section{References}

1 Agyeman AA, Ofori-Asenso R: Perspective: Does personalized medicine hold the future for medicine? J Pharm Bioallied Sci 2015;7:239-244.

2 Baum D: Personalized Marketing: 7 Impressive Examples \& Why They Worked 2018 [cited 2018 Mar 17]; Available from: https://www.impactbnd.com/blog/personalized-marketing-examples.

3 Share a Coke Names | Marketing Campaign | Coca-Cola GB [cited 2018 Mar 17]; Available from: http://www.coca-cola.co.uk/stories/share-a-coke.

4 Bromley P: Empowered individualism in world culture: Agency and equality in Canadian textbooks, 1871-2006. Eur J Cult Polit Sociol 2016;3:177-200.

5 Kennedy H: New Media's Potential for Personalization. Inf Commun Soc 2008;11:307-325.

6 Magids S, Zorfas A, Leemon D: The New Science of Customer Emotions. Harv Bus Rev 2015 [cited 2018 Mar 17]; Available from: https://hbr.org/2015/11/the-new-science-of-customer-emotions.

7 Gregg B, Kalaoui H, Maynes J, Schuler G: Marketing's Holy Grail: Digital personalization at scale | McKinsey \& Company [cited 2018 Mar 17]; Available from: https://www.mckinsey.com/business-functions/digital-mckinsey/our-insights/marketings-holy-grail-digital-personalization-atscale.

8 Collins F: Has the revolution arrived? Nature 2010;464:674-675.

9 Health (US) NI of, Study BSC: Understanding Human Genetic Variation. National Institutes of Health (US), 2007, [cited 2018 Mar 17]; Available from: https://www.ncbi.nlm.nih.gov/books/ NBK20363/.

10 Henney AM: The promise and challenge of personalized medicine: aging populations, complex diseases, and unmet medical need. Croat Med J 2012;53:207-10.

11 Sackett DL, Rosenberg WMC, Gray JAM, Haynes RB, Richardson WS: Evidence based medicine: what it is and what it isn't. BMJ 1996;312:71.

12 Rosenberg W, Donald A: Evidence based medicine: an approach to clinical problem-solving. BMJ 1995;310:1122-1126.

13 Schork NJ: Personalized medicine: Time for one-person trials. Nat News 2015;520:609.

14 Bereczki D: Personalized Medicine: A Competitor or an Upgrade of Evidence-based Medicine? Personalized Medicine 2012;9:211-221.

15 Kravitz RL, Duan N, Braslow J: Evidence-Based Medicine, Heterogeneity of Treatment Effects, and the Trouble with Averages. Milbank Q 2004;82:661-687.

16 Lehrach H: Virtual Clinical Trials, an Essential Step in Increasing the Effectiveness of the Drug Development Process. Public Health Genomics 2015;18:366-371.

17 Alhawassi TM, Krass I, Bajorek BV, Pont LG: A systematic review of the prevalence and risk factors for adverse drug reactions in the elderly in the acute care setting. Clin Interv Aging 2014;9:2079-2086.

18 Alomar MJ: Factors affecting the development of adverse drug reactions (Review article). Saudi Pharm J SPJ 2014;22:83-94.

19 Nyakutira C, Röshammar D, Chigutsa E, Chonzi P, Ashton M, Nhachi C, et al.: High prevalence of the CYP2B6 516G-->T(*6) variant and effect on the population pharmacokinetics of efavirenz in HIV/AIDS outpatients in Zimbabwe. Eur J Clin Pharmacol 2008;64:357-365.

20 Abrahams E, Ginsburg GS, Silver M: The Personalized Medicine Coalition: goals and strategies. Am J Pharmacogenomics 2005;5:345-355.

21 El-Sayeh HG, Morganti C: Aripiprazole for schizophrenia. Cochrane Database Syst Rev 2006;CD004578.

22 Gralnek IM, Dulai GS, Fennerty MB, Spiegel BMR: Esomeprazole versus other proton pump inhibitors in erosive esophagitis: a meta-analysis of randomized clinical trials. Clin Gastroenterol Hepatol 2006;4:1452-1458.

23 Mease PJ: Adalimumab in the treatment of arthritis. Ther Clin Risk Manag 2007;3:133-148. 
24 Ridker PM, MacFadyen JG, Fonseca FAH, Genest J, Gotto AM, Kastelein JJP, et al.: Number needed to treat with rosuvastatin to prevent first cardiovascular events and death among men and women with low low-density lipoprotein cholesterol and elevated high-sensitivity C-reactive protein: justification for the use of statins in prevention: an intervention trial evaluating rosuvastatin (JUPITER). Circ Cardiovasc Qual Outcomes 2009;2:616-623.

25 Cookson J, Gilaberte I, Desaiah D, Kajdasz DK: Treatment benefits of duloxetine in major depressive disorder as assessed by number needed to treat. Int Clin Psychopharmacol 2006;21:267-273.

26 Suissa S: Number needed to treat in COPD: exacerbations versus pneumonias. Thorax 2013;68:540-543.

27 Kristensen LE, Christensen R, Bliddal H, Geborek P, Danneskiold-Samsøe B, Saxne T: The number needed to treat for adalimumab, etanercept, and infliximab based on ACR50 response in three randomized controlled trials on established rheumatoid arthritis: a systematic literature review. Scand J Rheumatol 2007;36:411-417.

28 Freedman MS, Hughes B, Mikol DD, Bennett R, Cuffel B, Divan V, et al.: Efficacy of diseasemodifying therapies in relapsing remitting multiple sclerosis: a systematic comparison. Eur Neurol 2008;60:1-11.

29 Dekker A, Bulley S, Beyene J, Dupuis LL, Doyle JJ, Sung L: Meta-analysis of randomized controlled trials of prophylactic granulocyte colony-stimulating factor and granulocyte-macrophage colony-stimulating factor after autologous and allogeneic stem cell transplantation. J Clin Oncol Off J Am Soc Clin Oncol 2006;24:5207-5215.

30 Schee genannt Halfmann S, N Evangelatos N, Schröder-Bäck P, Brand A: European healthcare systems readiness to shift from ,one-size fits all' to personalized medicine. Personalized Medicine 2017; 14:63-74.

31 Evans WE, Relling MV: Pharmacogenomics: translating functional genomics into rational therapeutics. Science 1999;286:487-491.

32 Mukherjee D, Topol EJ: Pharmacogenomics in cardiovascular diseases. Prog Cardiovasc Dis 2002;44:479-498.

33 Jimmy B, Jose J: Patient Medication Adherence: Measures in Daily Practice. Oman Med J 2011;26:155-159.

34 Altshuler D, Daly MJ, Lander ES: Genetic mapping in human disease. Science 2008;322:881888.

35 Redekop WK, Mladsi D: The faces of personalized medicine: a framework for understanding its meaning and scope. Value Health 2013;16:4-9.

36 Munoz J, Kurzrock R: Targeted therapy in rare cancers--adopting the orphans. Nat Rev Clin Oncol 2012;9:631-642.

37 Pritchard DE, Moeckel F, Villa MS, McCarty CA, Housman LT, McCarty CA: Strategies for integrating personalized medicine into healthcare practice. Future Med 2017 [cited 2018 Mar 17]; Available from: https://www.futuremedicine.com/doi/pdf/10.2217/pme-2016-0064.

38 PerMed: Shaping Europe's Vision for Personalised Medicine - Strategic Research Innovation Agenda (SRIA) 2015 [cited 2017 Nov 9]; Available from: http://www.permed2020.eu/_media/PerMed_SRIA.pdf.

39 Hood L, Rowen L: The Human Genome Project: big science transforms biology and medicine. Genome Med 2013;5:79.

40 Rose N: Personalized Medicine: Promises, Problems and Perils of a New Paradigm for Healthcare. Procedia - Soc Behav Sci 2013;77:341-352.

41 Novelli G: Personalized genomic medicine. Intern Emerg Med 2010;5 Suppl 1:81-90.

42 Duarte TT, Spencer CT: Personalized Proteomics: The Future of Precision Medicine. Proteomes 2016;4:29.

43 Bush WS, Moore JH: Chapter 11: Genome-Wide Association Studies. PLOS Comput Biol 2012;8:e1002822. 
44 The 1000 Genomes Project Consortium: A map of human genome variation from populationscale sequencing. Nature 2010;467:1061-1073.

45 The 1000 Genomes Project Consortium: An integrated map of genetic variation from 1092 human genomes. Nature 2012;491:56-65.

46 The 1000 Genomes Project Consortium: A global reference for human genetic variation. Nature 2015;526:68-74.

47 Sudmant PH, Rausch T, Gardner EJ, Handsaker RE, Abyzov A, Huddleston J, et al:: An integrated map of structural variation in 2,504 human genomes. Nature 2015;526:75-81.

48 Ginsburg GS, McCarthy JJ: Personalized medicine: Revolutionizing drug discovery and patient care. Trends in Biotechnology 2001;19:491-496.

49 Santarosa M, Dolcetti R, Magri MD, Crivellari D, Tibiletti MG, Gallo A, Tumolo S, Della Puppa L, Furlan D, Boiocchi M, Viel A: BRCA1 and BRCA2 genes: role in hereditary breast and ovarian cancer in Italy. Int J Cancer 1999;83:5-9.

50 Mills RD, Mulhern TD, Cheng HC, Culvenor JG: Analysis of LRRK2 accessory repeat domains: prediction of repeat length, number and sites of Parkinson's disease mutations. Biochem Soc Trans 2012;40:1086-9.

51 Groden J, Thliveris A, Samowitz W, Carlson M, Gelbert L, Albertsen H, Joslyn G, Stevens J, Spirio L, Robertson M, et al.: Identification and characterization of the familial adenomatous polyposis coli gene. Cell 1991;66:589-600.

52 Schmalfuss F, Kolominsky-Rabas PL: Personalized Medicine in Screening for Malignant Disease: A Review of Methods and Applications. Biomark Insights 2013;8:9-14.

53 Henry NL, Hayes DF: Cancer biomarkers. Mol Oncol 2012;6:140-146.

54 Nagpal M, Singh S, Singh P, Chauhan P, Zaidi MA: Tumor markers: A diagnostic tool. Natl J Maxillofac Surg 2016;7:17-20.

55 Duffy MJ: Use of Biomarkers in Screening for Cancer. Adv Exp Med Biol 2015;867:27-39.

56 Verma M: Personalized Medicine and Cancer. J Pers Med 2012;2:1-14.

57 Nahta R: HER2-targeted therapies in breast cancer. Expert Opin Biol Ther 2013;13:949-952.

58 Wolff AC, Hammond ME, Schwartz JN, Hagerty KL, Allred DC, Cote RJ, Dowsett M, Fitzgibbons PL, Hanna WM, Langer A, McShane LM, Paik S, Pegram MD, Perez EA, Press MF, Rhodes A, Sturgeon C, Taube SE, Tubbs R, Vance GH, van de Vijver M, Wheeler TM, Hayes DF; American Society of Clinical Oncology/College of American Pathologists: American Society of Clinical Oncology/College of American Pathologists guideline recommendations for human epidermal growth factor receptor 2 testing in breast cancer. Arch Pathol Lab Med 2007;131:18-43.

59 Hudelist G, Pacher-Zavisin M, Singer CF, Holper T, Kubista E, Schreiber M, Manavi M, Bilban $\mathrm{M}$, Czerwenka K: Use of high-throughput protein array for profiling of differentially expressed proteins in normal and malignant breast tissue. Breast Cancer Res Treat 2004;86:281-91.

60 Gutiérrez-Xicoténcatl L, Plett-Torres T, Madrid-González CL, Madrid-Marina V: Molecular diagnosis of human papillomavirus in the development of cervical cancer. Salud Publica Mex 2009;51:Suppl 3:479-88.

61 Spear BB, Heath-Chiozzi M, Huff J: Clinical application of pharmacogenetics. Trends Mol Med 2001;7:201-4.

62 Abrahams E, Silver M: The Case for Personalized Medicine. J Diabetes Sci Technol 2009;3:680684.

63 Womersley K, Ripullone K: Medical schools should be prioritising nutrition and lifestyle education. BMJ 2017;359:j4861.

64 Borgiani P, Ciccacci C, Forte V, Sirianni E, Novelli L, Bramanti P, Novelli G: CYP4F2 genetic variant (rs2108622) significantly contributes to warfarin dosing variability in the Italian population. Pharmacogenomics 2009;10:261-6.

65 Interlandi J: The Paradox of Precision Medicine. Sci Am 2016;314:24.

66 Akhmetov I, Bubnov RV: Innovative payer engagement strategies: will the convergence lead to better value creation in personalized medicine? EPMA J 2017;8:5-15. 
67 Degtiar I: A review of international coverage and pricing strategies for personalized medicine and orphan drugs. Health Policy Amst Neth 2017;121:1240-1248.

68 Cordeiro JV: Ethical and legal challenges of personalized medicine: Paradigmatic examples of research, prevention, diagnosis and treatment. Rev Port Saúde Pública 2014;32:164-180.

69 Sloan RP, Bagiella E, Powell T: Religion, spirituality, and medicine. The Lancet 1999;353:664667.

70 Levitt M: The Ethics of Personalised Medicine: Critical Perspectives. Med Law Rev 2016;24:306309.

71 Gravitz L: Therapy: This time it's personal. Nature 2014 [cited 2018 Mar 17]; Available from: https://www.nature.com/articles/509S52a.

72 Becker F, van El CG, Ibarreta D, Zika E, Hogarth S, Borry P, et al.: Genetic testing and common disorders in a public health framework: how to assess relevance and possibilities. Eur J Hum Genet 2011;19:6-44.

73 Horgan Richard P, Kenny Louise C: "Omic" technologies: genomics, transcriptomics, proteomics and metabolomics. Obstet Gynaecol 2011;13:189-195.

74 Leyens L, Horgan D, Lal JA, Steinhausen K, Satyamoorthy K, Brand A: Working towards personalization in medicine: main obstacles to reaching this vision from today's perspective. Per Med 2014;11:641-649.

75 Alyass A, Turcotte M, Meyre D: From big data analysis to personalized medicine for all: challenges and opportunities. BMC Med Genomics 2015;8:33.

76 European Commission: Biobanks for Europe. A challenge for governance, Belgium 2012.

77 European Commission: Use of "-omics" Technologies in the Development of Personalised Medicine, Belgium 2013.

78 European Commission - Directorate-General for Health and Food Safety: Personalised medicine 2018 [cited 2018 Mar 17]; Available from: https://ec.europa.eu/health/human-use/personalisedmedicine_en.

79 Nimmesgern E, Norstedt I, Draghia-Akli R: Enabling personalized medicine in Europe by the European Commission's funding activities. Pers Med 2017;14:355-365.

80 European Commission: Towards an International Consortium for Personalised Medicine (IC PerMed) 2016.

81 Diaz JA, Griffith RA, Ng JJ, Reinert SE, Friedmann PD, Moulton AW: Patients' Use of the Internet for Medical Information. J Gen Intern Med 2002;17:180-185.

82 Mählmann L, Röcke C, Brand A, Hafen E, Vayena E: Attitudes towards personal genomics among older Swiss adults: An exploratory study. Appl Transl Genomics 2016;8:9-15.

83 Abul-Husn NS, Owusu Obeng A, Sanderson SC, Gottesman O, Scott SA: Implementation and utilization of genetic testing in personalized medicine. Pharmgenomics Pers Med 2014;7:227240.

84 Ogino S, Fuchs CS, Giovannucci E: How many molecular subtypes? Implications of the unique tumor principle in personalized medicine. Expert Rev Med Diagn 2012;12:621-628.

85 Egglestone C, Morris A, O'Brien A: Effect of direct-to-consumer genetic tests on health behaviour and anxiety: a survey of consumers and potential consumers. J Genet Couns 2013;22:565-575.

86 Su P: Direct-to-Consumer Genetic Testing: A Comprehensive View. Yale J Biol Med 2013;86:359-365.

87 Bloss CS, Wineinger NE, Darst BF, Schork NJ, Topol EJ: Impact of direct-to-consumer genomic testing at long term follow-up. J Med Genet 2013;50:393-400.

88 Vayena E, Ineichen C, Stoupka E, Hafen E: Playing a Part in Research? University Students' Attitudes to Direct-To-Consumer Genomics. Public Health Genomics 2014;17:158-168.

89 Vayena E, Salathé M, Madoff LC, Brownstein JS: Ethical Challenges of Big Data in Public Health. PLOS Comput Biol 2015;11:e1003904.

90 Gollust SE, Gordon ES, Zayac C, Griffin G, Christman MF, Pyeritz RE, et al.: Motivations and Perceptions of Early Adopters of Personalized Genomics: Perspectives from Research Participants. Public Health Genomics 2012;15:22-30. 
91 Wasson K, Sanders TN, Hogan NS, Cherny S, Helzlsouer KJ: Primary care patients' views and decisions about, experience of and reactions to direct-to-consumer genetic testing: a longitudinal study. J Community Genet 2013;4:495-505.

92 Ashley DeVan: The 7 V's of Big Data 2016 [cited 2017 Nov 1]; Available from: https://www.impactradius.com/blog/7-vs-big-data/.

93 National Information Board - HM Government: Personalised Health and Care 20202014 [cited 2017 Nov 1]; Available from: https://www.gov.uk/government/uploads/system/uploads/attachment_data/file/ 384650/NIB_Report.pdf.

94 MähImann L, Reumann M, Evangelatos N, Brand A: Big Data for Public Health Policy-Making: Policy Empowerment. Public Health Genomics 2018; DOI: 10.1159/000486587.

95 Andersen V, Holmskov U, Sørensen SB, Jawhara M, Andersen KW, Bygum A, Hvid L Grauslund J, Wied J, Glerup H, Fredberg U, Villadsen JA, Kjær SG, Fallingborg J, Moghadd SAGR, Knudsen T, Brodersen J, Frøjk J, Dahlerup JF, Nielsen OH, Christensen R, Bojesen AB, Sorensen GL, Thiel S, Færgeman NJ, Brandslund I, Stensballe A, Schmidt EB, Franke A, Ellinghaus D, Rosenstiel P, Raes J, Heitmann B, Boye M, Nielsen CL, Werner L, Kjeldsen J, Ellingsen T: A Proposal for a Study on Treatment Selection and Lifestyle Recommendations in Chronic Inflammatory Diseases: A Danish Multidisciplinary Collaboration on Prognostic Factors and Personalised Medicine. Nutrients 2017;9(5). DOI: 10.3390/nu9050499.

96 Kingsley MJ, Abreu MT: A Personalized Approach to Managing Inflammatory Bowel Disease. Gastroenterol Hepatol (NY) 2016;12:308-315.

97 Wiley: The Long March to Personalized Medicine in IBD Treatment. Key Opinions in Medicine $2017 ; 1$.

98 Colombel J-F, Feagan BG, Sandborn WJ, Van Assche G, Robinson AM: Therapeutic drug monitoring of biologics for inflammatory bowel disease. Inflamm Bowel Dis 2012;18:349-358.

99 McGovern D: Personalized Medicine in Inflammatory Bowel Disease. Gastroenterol Hepatol (NY) 2014;10:662-664.

100 Cosnes J: Smoking, physical activity, nutrition and lifestyle: environmental factors and their impact on IBD. Dig Dis 2010;28:411-417.

101 Narula N, Fedorak RN: Exercise and inflammatory bowel disease. Can J Gastroent 2008;22:497504.

102 Mählmann L, Gerber M, Furlano RI, Legeret C, Kalak N, Holsboer-Trachsler E, Brand S: Psychological wellbeing and physical activity in children and adolescents with inflammatory bowel disease compared to healthy controls. BMC Gastroenterol 2017;17:160.

103 Mählmann L, Gerber M, Furlano RI, Legeret C, Kalak N, Holsboer-Trachsler E, Brand S: Impaired objective and subjective sleep in children and adolescents with inflammatory bowel disease compared to healthy controls. Sleep Med 2017;39:25-31.

104 Mählmann L, Gerber M, Legeret C, Köhler H, Kalak N, Holsboer-Trachsler E, Brand S, Furlano $\mathrm{R}$ : Favorable impact of chronic aerobic exercise training on disease symptoms in children and adolescents with inflammatory bowel disease. About to be submitted 2018.

105 Mählmann L, Gerber M, Furlano RI, Legeret C, Kalak N, Sadeghi Bahmani D, Holsboer-Trachsler E, Brand S: Aerobic exercise training in children and adolescents with inflammatory bowel disease: Influence on psychological functioning, sleep and physical performance. Eur Psychiatry 2017;41:125.

106 Hayes DF, Markus HS, Leslie RD, Topol EJ: Personalized medicine: risk prediction, targeted therapies and mobile health technology. BMC Med 2014;12:37.

107 Swan M: Health 2050: The Realization of Personalized Medicine through Crowdsourcing, the Quantified Self, and the Participatory Biocitizen. J Pers Med 2012;2:93-118.

108 Markoff J: The iPad in your hand: As fast as a supercomputer of yore. 2011 [cited 2018 Jan 25]; Available from: http://bits.blogs.nytimes.com/2011/05/09/the-ipad-in-your-hand-as-fast-as-a-supercomputer-of-yore/?_php=true\&_type=blogs\&_r=0.

109 Ericsson: Ericsson Mobility Report 2017 [cited 2018 Jan 21]; Available from: https://www.ericsson.com/en/mobility-report/reports/november-2017. 
110 Steinhubl SR, Muse ED, Topol EJ: The emerging field of mobile health. Sci Transl Med 2015;7:283rv3.

111 WHO: mHealth: New horizons for health through mobile technologies: second global survey on eHealth. 2011 [cited 2018 Jan 25]; Available from: http://www.who.int/goe/publications/goe_mhealth_web.pdf.

112 Zhao J, Freeman B, Li M: Can Mobile Phone Apps Influence People's Health Behavior Change? An Evidence Review. J Med Internet Res 2016;18:e287.

113 Pate RR, Freedson PS, Sallis JF, Taylor WC, Sirard J, Trost SG, et al.: Compliance with physical activity guidelines: prevalence in a population of children and youth. Ann Epidemiol 2002;12:303-308.

114 Rees S, Williams A: Promoting and supporting self-management for adults living in the community with physical chronic illness: A systematic review of the effectiveness and meaningfulness of the patient-practitioner encounter. JBI Libr Syst Rev 2009;7:492-582.

115 Ory MG, Smith ML, Mier N, Wernicke MM: The Science of Sustaining Health Behavior Change: The Health Maintenance Consortium. Am J Health Behav 2010;34:647-659.

116 Mählmann L: MoVit - ICT 4 Good Fellowship Application 2016.

117 WHO: Goals of a health system 2010 [cited 2018 Jan 25]; Available from: http://www.wpro.who.int/health_services/action/regional_strategy_health_systems_primary_health_care_chapter3.pdf.

118 Sultana J, Cutroneo P, Trifirò G: Clinical and economic burden of adverse drug reactions. J Pharmacol Pharmacother 2013;4:73-77.

119 Schee Genannt Halfmann S, Mählmann L, Leyens L, Reumann M, Brand A: Personalized Medicine: What's in it for Rare Diseases? Adv Exp Med Biol 2017;1031:387-404.

120 Haeusermann T, Greshake B, Blasimme A, Irdam D, Richards M, Vayena E: Open sharing of genomic data: Who does it and why? PLOS ONE 2017;12:e0177158.

121 Personalised Medicine Conference 2016. Eur Comm - [cited 2018 Apr 10]; Available from: https://ec.europa.eu/info/events/personalised-medicine-conference-2016-2016-jun-01_en.

122 Vayena E, Tasioulas J: "We the Scientists": a Human Right to Citizen Science. Philos Technol 2015;28:479-485.

123 Universal Declaration of Human Rights 2015 [cited 2018 Mar 18]; Available from: http://www.un.org/en/ universal-declaration-human-rights/.

124 Abrams LR, McBride CM, Hooker GW, Cappella JN, Koehly LM: The Many Facets of Genetic Literacy: Assessing the Scalability of Multiple Measures for Broad Use in Survey Research. PLOS ONE 2015;10:e0141532.

125 Minich DM, Bland JS: Personalized lifestyle medicine: relevance for nutrition and lifestyle recommendations. ScientificWorldJournal 2013:129841.

126 Fuster V: An alarming threat to secondary prevention: low compliance (lifestyle) and poor adherence (drugs). Rev Espanola Cardiol Engl Ed 2012;65 Suppl 2:10-16.

127 Johnson D, Deterding S, Kuhn K-A, Staneva A, Stoyanov S, Hides L: Gamification for health and wellbeing: A systematic review of the literature. Internet Interv 2016;6:89-106.

128 Xian Y, Xu H, Xu H, Liang L, Hernandez AF, Wang TY, et al.: An Initial Evaluation of the Impact of Pokémon GO on Physical Activity. J Am Heart Assoc 2017;6. DOI: 10.1161/JAHA.116.005341.

129 mHealthIntelligence: Telemedicine Robots: Out of Science Fiction and Into the Mainstream. mHealthIntelligence 2017 [cited 2018 Mar 19]; Available from: https://mhealthintelligence.com/features/can-telemedicine-robots-move-from-fantasy-to-fact.

130 Hughes-Wilson W, Palma A, Schuurman A, Simoens S: Paying for the Orphan Drug System: break or bend? Is it time for a new evaluation system for payers in Europe to take account of new rare disease treatments? Orphanet J Rare Dis 2012;7:74.

131 Garrison LP, Towse A: Value-Based Pricing and Reimbursement in Personalised Healthcare: Introduction to the Basic Health Economics. J Pers Med 2017;7. DOI: 10.3390/jpm7030010. 
132 Institute of Medicine (US): Policy Changes to Improve the Value We Need from Health Care. National Academies Press (US), 2008, [cited 2018 Apr 10]. Available from: https://www.ncbi.nlm.nih.gov/books/ NBK52830/.

133 Teich ST, Faddoul FF: Lean Management - The Journey from Toyota to Healthcare. Rambam Maimonides Med J 2013;4. DOI: 10.5041/RMMJ.10107.

134 Rotter T, Plishka CT, Adegboyega L, Fiander M, Harrison EL, Flynn R, et al.: Lean management in health care: effects on patient outcomes, professional practice, and healthcare systems: The Cochrane Library. John Wiley \& Sons, Ltd, 2017, [cited 2018 Apr 10]. Available from: http://cochranelibrary-wiley.com/doi/10.1002/14651858. CD012831/full.

135 Curatolo N, Lamouri S, Huet J-C, Rieutord A: A critical analysis of Lean approach structuring in hospitals. Bus Process Manag J 2014;20:433-454.

136 Kaplan GS, Patterson SH, Ching JM, Blackmore CC: Why Lean doesn't work for everyone. BMJ Qual Saf 2014;23:970-973. 



\section{Valorisation}



This dissertation "Lean Personalized Medicine? From genetic reductionism towards holistic Personalized Medicine," addresses the challenge of broadening the paradigm of PM again and acknowledges the highly interactive systems approach necessary for truly personalization in health care. This chapter on knowledge valorization refers to the translation of scientific knowledge into the practical societal value. The value can have a variety of formats including competitive products, services, processes and new commercial activities. Accordingly, placing scientific activity in an economic, social or environmental context can create more legitimacy and added value of the research activities. Therefore, the aim of this chapter is to describe how the scientific findings reported earlier can yield benefits for different societal stakeholders beyond its value to the scientific community.

\section{Relevance of the Topic}

Personalized Medicine (PM) is the response towards the need of a more tailormade approach in medicine to provide each individual patient with the right treatment, the right dosage at the right time. Current first success stories are mainly reported in the field of oncology. Targeted testing for specific genetic abnormalities has been transforming the classification and treatment. However, development and implementation of Personalized Medicine approaches for other diseases and many aspects of health care delivery are still far from being reality. Genetic effects in isolation are insufficient to identify the risk in the most prevalent disease and it is actually needed to integrate genetic and epigenetic effects with environmental exposure as well as lifestyle components. Genetics are just one of a suite of personalized tools to achieve healthier living, rather than an almighty method to predict future disease. Therefore, this dissertation shed light on additional translational pathways towards holistic personalized medicine.

\section{Target groups}

As the results are presented in this dissertation, they might be of interest for multiple stakeholders of the health care system including citizens/ patients, health care professionals and policy makers. In the following I will illustrate how each of the target groups is addressed or assessed in each of the parts of this dissertation. Overall, all projects of the dissertation are directly or indirectly patient centered.

The first target group of this dissertation are citizens as stakeholders in society and in their role of (potential future) patients. In Part 1 understanding people's 
awareness, attitudes, motivation and concerns about personal genomic testing, accessing genetic data and genetic data sharing is in focus. The assessment provides the chance to integrate the broader public into the debate and ultimately gives insights on how to protect individual rights, like privacy and confidentiality, while avoiding undue paternalism that potentially prohibits (scientific) development. In Part 2 and 5, the proposed solution of HDC put "citizens in the driver's seat" to take control and ownership over their data. Through increased empowerment of patients due to access to health data and increased health literacy patients have to be acknowledge as partners in the decision making process. There is a need to address the limits of a top-down and expert-based approach. Part 3 and 4 both investigate how to improve patients' self-management abilities through the integration of lifestyle. All stakeholders will benefit by the increasing awareness of the importance of physical activity among inactive people and the accompanied health benefits. Furthermore, with the mobile health application "MoVit" a direct solution has been proposed to overcome the challenge of behavior change towards a physically active lifestyle.

The second target group is policy makers. As described in Part 1, given the overall need for genomic data, the interest that people are showing in accessing their genomic data and even donating them for research, as well as the concerns about the practices of many personal genomics providers an in depth understanding is crucial for developing appropriate and evidence-informed policies and practices in relation to recruitment, informed consent and privacy setting options relevant to personal genomics. As these are new developments, we are only in the beginning of the debate about what measures policy makers need to take at the regulatory level, how to engage the broader public in the debate and ultimately how to protect individual rights while avoiding undue paternalism. Furthermore, as we move to Part 2 the proposed solution of HDCs lead to the need of data sharing regulations on an international level. Therefore, it becomes even more complex to develop global policies responsive to different cultural perceptions about data sharing, different levels of trust to the research enterprise including different health care systems and data protection laws. At the same time, integrating Big Data and Health Data Cooperatives (HDCs) is expected to lead to highly valuable insights that will inform and empower policy making. In sum, on one side, policy makers are challenged by the new regulatory requirements needed for data protection, while on the other side they might strongly increase their effectiveness and impact due to the option of data driven policy making.

Furthermore, Health Care Professionals are the third target group of this dissertation. Health care professionals have always wanted to offer their patients the best treatment to suit their specific case. To fulfill this goal, roles of health care professionals might have very different formats. In Part 1 health professionals are reported as a missing piece in a successful interaction of lay people with their 
genetic data. DTC-GT companies have frequently been criticized to not provide their customers with professional support after receiving their results. This might lead to an increased health care consumption due to unsettled patients who need support in understanding and wish confirmation of test results. Furthermore, access to genetic and online health data will empower patients and they might challenge the health professionals' treatment decisions or question their authority. Finally, health care professionals will be confronted with (genetic) data they might not be familiar with and unable to integrate this knowledge into their practice. In Part 2 the solution of a HDC will give the health care professional access to a complete set of patient data. The reproduction of previous data points (images, scans, samples etc.) might be obsolete. Furthermore, the integration of new data points (eg. lifestyle data) might lead to new insights and answers to questions that have previously been beyond reach. Part 3 and 4 aim to raise the health care professionals' attention towards the importance of lifestyle as a significant predictor in treatment success. The proposed tool "MoVit" enables and supports the health professional to prescribe organized lifestyle changes and preventive treatment and personal activity reports provide an additional continuous source of information for medical decision making. Finally, Part 5 illustrates how the in depth knowledge on molecular characteristics of patients will lead towards a new paradigm of disease categorization. Soon every patient will be categorized as unique and cannot be treated according to average-based data.

\section{Activities/Products}

Building on the previous sections, the results of this dissertation have the potential to be translated and shaped into several concrete products, services or activities:

Findings of Part 1 aim to inform policy makers and can be translated to potential policy recommendations on how to protect people's values and rights while enabling ethically sound research and data sharing activities to support scientific advancement. Furthermore, the potential of citizen science initiated by public institutions has been discussed in detail and would be a great opportunity for further action taking. In Part 2 and Part 5 the solution of HDCs is discussed in detail: HDCs are unified data systems that promote data access and data linkage of heterogeneous data from a multitude of sources from within and outside of the health domain, while still citizen owned. They offer a framework to overcome the technical, political and societal challenges and to empower policy making by the integration and application of Big Data. Part 3 illustrates the potential of lifestyle interventions in chronic complex diseases and Part 4 gives a concrete solution on how to integrate lifestyle by the use of a mobile app solution "MoVit". 
Prevention of adverse health outcomes may have an enormous impact on future health care budgets, since these are strongly associated with mortality and longterm morbidity.

\section{Innovation}

Overall the dissertation is illustrating translational aspects of the innovative paradigm of PM going beyond genetic reductionism and integrating a holistic approach with the patient at center. The above suggested products and services can be called innovative in respect to the existing range of products, services and activities in various ways:

1. "Citizen science" as a concept that will lead towards an improved dialogue between science and society, as well as the integration of society into the process of co-creating scientific knowledge.

2. Health Data Cooperatives (HDC) and the integration of multiple sources of data on an international level, which will lead to new insights and increased effectiveness in health care and beyond.

3. The innovative model of personalized lifestyle medicine has been introduced: a model enabling lifestyle oriented treatment strategies to improve health outcomes based on individuals health metrics and personal preferences.

4. "MoVit" a physical activity health app which translates scientific evidence of behaviour change into a personalized physical activity coaching program and mobile application to provide health care professionals with a tool to promote an active lifestyle among their patients.

5. Overall the integration of PM will lead towards a new perception on how we define certain disease categories and put the patient at center.

The different valorization proposals as outlined above all have a very different timeline and scope for implementation. While the mobile health application has been followed up and has been pitched and prototyped in different contexts, the concept of integrating citizen science into academic research, the proposed HDCs and the integration of lifestyle medicine need a long-term approach. 


\section{Summary}



This dissertation aims to shed light on translational aspects to move from genetic reductionism toward holistic systems thinking in personalized medicine (PM). Summarized the approach will shed light on (1) the development of societal awareness and ethical argumentations to inform political decision making, (2) the integration of Big Data and ICT in the development of PM, (3) practical applications investigated in the context of chronic complex disease, (4) the inclusion of lifestyle and its preventive potential when applied in a personalized manner and (5) the overall challenges on the path towards a sustainable health care system.

According to the definition of the European Commission Horizon 2020 Advisory Group for Societal Challenge, "Health, Demographic Change and Wellbeing" PM refers to "a medical model using characterization of individuals' phenotypes and genotypes (e.g. molecular profiling, medical imaging, lifestyle data) for tailoring the right therapeutic strategy for the right person at the right time, and to determine the predisposition to disease and deliver timely and targeted prevention."

Current first success stories are mainly reported in the field of oncology. Targeted testing for specific genetic abnormalities has been transforming the classification and treatment. However, development and implementation of PM approaches for other diseases and many aspects of health care delivery are still far from being reality. Genetics are just one of a suite of personalized tools to achieve healthier living, rather than an almighty method to reliable predicts future disease. Despite various calls for a more holistic view of PM, this term or alternatively system medicine remain proxies for the application of genetics to individualized therapies. In the present dissertation, the five SRIA of challenges have been chosen as a frame of orientation and horizontal guideline. The aim is to fill the challenges with scientific investigations, original data and specific examples, to shed light on translations aspects in order to move from a genetic focus towards more holistic systems thinking in PM.

\section{Developing Awareness and Empowerment: Case of Personal Genomics}

Given the overall need for genomic data to receive further insights into the complex system of health and disease, the first objective was to investigate the societal perception of research participation, the value people attribute to genetic research and the types of trade-offs between privacy and utility that people are willing to accept.

In Part 1, attitudes of Swiss older adults towards personal genomics and peoples' willingness to engage and share their genetic data for research have been explored. The study indicated a positive overall attitude towards personal genomic testing among older Swiss adults, a group not typically represented in 
studies about personal genomics. Main drivers were the possibility to access their personal disease risk and the opportunity to contribute to scientific research. Based on Part 1 we can conclude, that an increased focus on general public engagement, especially of older adults, in scientific research activities known as "citizen science" initiated by public research institutions may further strengthen the uptake of PM.

\section{Big Data}

Part 2 illustrates how to integrate the infinite amount of emerging data, known as Big Data to support the development the specific cases of policy making. For effective, high quality policy making in the context of public health we need to reshape the process of policy making using continuous, real time data inflow. In detail, the objective of Part 2 was to investigate the potential of Big Data as a support tool for making innovative decisions in public health policy making. Based on the findings, it has been concluded that the value and necessity of a panEuropean data sharing platform or (Health) Data Cooperatives is its function as a tool to tap into the potential of all existing and rapidly emerging data sources. Such a Data Common would enable to leverage all the data required to overcome computational, algorithmic and technological challenges that characterize today's highly heterogeneous data landscape, as well a host of diverse regulatory, normative, governance and policy constraints.

\section{Translating Basic to Clinical Research and Beyond: Chronic Complex Disease}

A majority diseases is caused by lifestyle issues: Nutrition, exercise and environment. However, medicine has not yet tapped into this potential. The need for PM derives from the realization that today's most challenging medical conditions are chronic complex diseases with multiple pathogenic components that interact with each other. Currently, complex diseases cannot properly be controlled and much less cured by modulating single components at sporadic time points in the course of the disease or administering the same treatment to all patients. Lifestyle interventions namely physical activity have been tested as a promising intervention in a large variety of chronic inflammatory diseases to improve inflammation along with sleep quality, mental well-being, fitness and daily physical activity. Therefore, objective 3 was to explore the influence of a lifestyle intervention including regular physical activity on inflammation, fitness, depression and sleep among pediatric patients with IBD. In summary, the four articles showed that participants with IBD 
in an active state of the disease (IBD-AD) showed higher markers of inflammation, had lower psychological functioning, impaired objective sleep and lower PA (average steps per day) compared to participants with IBD in remission (IBD-RE) and healthy controls $(\mathrm{HC})$. After an 8-week physical activity intervention exercise capacity improved in all participants, self-reported fitness and daily physical activity increased in IBD-AD, but not in IBD-RE and HC. No improvements were observed for psychological functioning, depressive symptoms or subjective sleep. Objective sleep improved, deep sleep increased, light sleep decreased, and in the IBD-AD group the number of arousals after sleep onset also decreased. Finally, after a single bout of exercise inflammation markers strongly increased in all three groups, while after a long-term AET intervention, inflammation significantly decreased.

In conclusion, the integration of lifestyle into medical practice is one step to add and provide additional treatment options and recognize the importance of lifestyle and behavior change as an integral part and opportunity to increase treatment effectiveness and reduce costs. The increased efficacy through personalization will lead to empowerment, feeling of self-efficacy and regaining of control for chronically ill patients to improve health outcomes based on individual health metrics and personal preferences.

\section{Bridging Innovation to the Market: mHelath and Business opportunities}

A crucial tool in terms of innovation and development towards the notion of PM is mobile health (mhealth). The convergence of wearable electronics, miniaturized sensor technologies, and mobile phone penetration provides novel opportunities for personalized lifestyle interventions and support of health care practice. For example, the positive effects of regular physical activity are well known, yet many people do not comply with physical activity guidelines. General practitioners and other medical professionals often don't have time to encourage inactive patients or personalize their lifestyle advice to the patient's needs. As soon as people start moving, it is important to maintain this behavior, a second barrier many people fail. Physical Activity Behavior is highly dynamic, momentary and embedded in context. With a business plan, Part 4 will propose a tool to translate scientific evidence on behavior change into a personalized program to support GPs and empower patients to start a physically active life. Aiming towards holistic $\mathrm{PM}, \mathrm{MoV}$ it is a concrete example on how to empower patients and health care professionals to integrate evidence based lifestyle advices into clinical practice. MHealth again, produces data that might improve patient self-efficacy, health literacy and may contribute towards decision making in the health care context. 


\section{Shaping Sustainable Healthcare: Discussion and Future scenarios}

With the promise of PM to improve outcomes by providing treatment to the right patient, at the right dosage, at the right time and avoiding the trial and error based traditional "one-size fits all" approach, new opportunities emerged to reach the health care systems goals. Understandably, this development is expected to reduce the economic burden health care systems are facing due to adverse treatment effects, incomplete benefits or misdiagnosis. At the same time, the changing medical model will create new demands on the health care system. Objective 5 was to investigate the role of health care systems and policy making, when shifting focus from traditional disease categorization towards unique disease profiles.

In conclusion for Part 5, as well as overall, the implementation of PM will need a paradigm shift from multiple stakeholders and the health care system towards holistic systems thinking to realize truly PM. Technology will be the main driver in the fast development. However, patient-centeredness should be the ultimate goal of personalization. Therefore, the paradigm of PM should remain flexible and dynamic to integrate exponentially growing technology, incorporate lifestyle and environmental medicine and place the patient at center: Lean Personalized Medicine. 
Acknowledgements 

It always seems impossible, until it is done.

\section{Nelson Mandela}

... and as soon as it was done, I was, and still am, overwhelmed by the feeling of relief and thankfulness, for all the people in my surrounding who made the impossible, possible and supported and contributed to this work in all different ways: Content wise, emotionally or multiple other ways. Conducting this PhD was a challenge for me and I am proud to sit here in this moment and bring this chapter to an end. In the following, I would like to thank the people who contributed in the language I usually speak to them.

In diesem Zusammenhang möchte ich zuallererst dir, liebe Angela, von Herzen danken. Du, als meine Doktormutter, hast natürlich einen ganz besonderen Beitrag zu dieser Arbeit geleistet und ich danke dir für die Möglichkeiten die du auf verschiedenste Weise für mich ins Leben gerufen hast. Außerdem danke ich dir für deine Offenheit, Herzlichkeit und Bemühungen in allen Lebenslagen. Du hast in mir den Samen einer Vision gepflanzt, die ich auch über diese Arbeit hinaus verfolgen werde. Und du hast mir neue Wege und Vorgehensweisen gezeigt und mich immer wieder durch deine Standhaftigkeit und Überzeugungskraft inspiriert.

Als zweites möchte ich ganz besonders bei meinem IBD-Studien Team in Basel bedanken: Lieber Serge, ich habe in den letzten drei Jahren unglaublich viel von dir gelernt und bin dir sehr, sehr dankbar dafür. Du bist der beste Supervisor den ich mir hätte wünschen können. Deine Art zu motivieren, unterstützen aber auch zu kritisieren und verbessern hat mir unglaublich geholfen. Dein Beitrag zu dieser Arbeit ist wirklich essentiell und wurde durch deine Unterstützung und deinen kritischen Blick nochmal auf ein ganz neues Niveau gebracht. Lieber Markus, lieber Raoul und liebe Frau Prof. Dr. Holsboer, auch euch möchte ich ganz herzlich danken. Es war ein tolles Teamwork, dass wir auf die Beine gestellt haben und ich danke euch für eure konstante Unterstützung, Bereitschaft und eure lobenden Worte auch bei kleinen Erfolgen. Diese Details haben mich immer wieder besonders motiviert und haben des öfteren weitere 10-20 \% Einsatzkraft aktiviert. Lieber Nadeem, Corinne und Noe, auch euch wollte ich hiermit herzliche danken. In verschiedenen Elementen habt ihr mich begleitet und unterstützt und ich habe immer sehr sehr gerne mit euch zusammengearbeitet. Liebe Marielle und lieber Vladimir, euch gilt auch ein ganz besonderer Dank. Ihr habt mir geholfen diverse Schlafdaten zu analysieren. Mit Hilfe eures Einsatzes haben wir nochmal einen ganz besonderen Fokus auf den Schlaf legen können, der uns ein unerwartetes weiteres Paper beschert hat. Außerdem freue ich mich immer, wenn ich bei euch reinschauen kann und herzlich empfangen werde. All in all: Danke IBD-Team. Wir waren wirklich ein tolles Team. Außerdem darf ich nicht vergessen, dass das Forscherteam natürlich nur die eine Seite der Medaille ist. Daher möchte ich an 
dieser Stelle auch ein ganz herzliches Danke an alle Kinder, Jugendliche und Eltern der IBD Studie aussprechen. Ich denke euch allen für euer Engagement und eure Teilnahme. Mit euch hat es ganz besonders viel Spaß gemacht.

Als drittes möchte ich mich ganz herzlich bei meinem IBME Institute in Zürich und der ETH bedanken. Das erste Projekt dieser Arbeit ist zusammen mit Effy Vayena und Ernst Hafen in Zürich entstanden und hat mir einen spannenden Eindruck in die ethischen Hintergründe der Personalisierten Medizin gegeben. Ganz besonders möchte ich mich hier auch bei Christian Ineichen bedanken. Christian, du bist für mich ein Forscher aus dem Bilderbuch und ein unglaublich wertvoller Kollege. Ich danke dir, für deinen Support und freue mich, dass unsere Zusammenarbeit auch noch über mein Praktikum hinaus zu netten Abenden im Niederdörfli geführt hat. Außerdem möchte ich mich auch im Rahmen dieses Projektes nochmals herzlichst bei allen Teilnehmern der Studie danken: Den Mitgliedern der Seniorenuniversität. Der Austausch, das Engagement und auch die Offenheit in den durchgeführten Befragungen und Interviews waren ganz besonders wertvoll.

Fourth, I would like to thank the PhD student team of the Brand family. Of course this team was a crucial factor in the development of this thesis. First of all, Basti, my doctor brother: We spend a lot of emails and phone calls together and successfully published two papers. Furthermore, Basti was always a great colleague, well-structured and best partner if you want to get things done. Thank you for being my back entrance to anything administrative or information on where to currently find Angela, as well as your openness for any content or emotional exchange. Second, I would like to thank Matthias, my Big Data expert and second doctor brother who almost became my law-consultant at one point. Thank you very much for your constant support in so many cases, your warm words and understanding. Next, I would like to thank Lada, Timo, Nikos and Phil. It was a great to get to known all of you and spend a very nice weekend in the Switzerland. Finally, I also would like to thank Helmut Brand and Peter Schröder for your support. I have the feeling that some very good strategic advices that I got from Angela were informed and inspired by you and I would like to thank you for this direct and indirect support. I also would like to thank Anita who strongly supported the final steps on this journey.

Dann möchte ich mich ganz herzlich bei meinem UPK Brain Gut and Depression Team bedanken. Allen voran meinen Kollegen André und Cedric, und auch Undine Lang, Stefan Borgwardt, Serge Brand und Christoph Beglinger. Wir haben in den letzten 1.5 Jahren zwei sehr spannende Projekte auf die Beine gestellt und ich bin jetzt schon gespannt, wo es uns hinführen wird.

Dem DRFZ in Berlin möchte ich auch ganz herzlich für das Obdach in meiner Berliner Zeit danken. Da ich in der Endphase meiner Dissertation parallel in Potsdam eine Design Thinking Ausbildung absolviert habe, brauchte ich noch einen 
Platz um ab und zu einmal ein Paper herunterladen zu können. Vielen Dank für eure herzliche Aufnahme.

Als nächstes möchte ich all meinen lieben Freunden danken, die dafür gesorgt haben, dass es auch außerhalb meiner Arbeit immer wieder gut abgelenkt werde.

Als erstes, möchte ich einer meiner größten Stütze in so ziemlich jeder Lebenslage danken: Claudi. Wie froh bin ich, dass es dich und Malte in meine Nähe verschlagen hat und wie unglaublich dankbar bin ich dir für dein stets offenes Ohr, die vielen spannenden gemeinsamen Erlebnisse und ganz besonders deine emotionale Unterstützung. Du bist mir in vielen Dingen eine Vorbild. Ganz besonders im Zusammenhang mit dieser Arbeit danke ich dir für deine wertvollen inhaltlichen Kommentare und Hinweise. Und im selben Atemzug danke ich auch dir lieber Malte. Ich freue mich, dass Volleyball uns irgendwann mal in Kontakt gebracht hat und ich freue mich, dass wir herausgefunden haben, dass uns noch so viel mehr verbindet.

Meine liebe Katja, auch dir möchte ich ganz herzlich danken. Vor Allem dafür, dass du einer der Menschen bist, die die Welt manchmal ein bisschen anders sehen und mir dadurch immer wieder Denkstoff geben und mich aus meiner Komfortzone treiben. Deine Ratschläge sind nicht immer die, die man hören will... aber immer die, die wirklich helfen. Danke dir dafür und noch für viel mehr.

Meine lieben Jungs Wolf, Lolo und Niculin, kein Samstag in Zürich ist so schön mit euch auf dem Flohmi oder bei einem Cappuccino. Keine Herzen sind so groß wie Eure. Ich danke euch dafür, dass euch kein Wasser zu kalt ist zum reinspringen und man mit euch so herrlich viel Spass haben kann. Außerdem danke ich euch, für euren starken emotionalen Support: In Flühli, unterm Sternenhimmel und euren tollen kreativen Input, der beispielsweise die Titelseite stark beeinflusst hat.

Meine kleine adoptierte Schwester Anna: mein Lieblingsmitbewohnerin, Seelenverwandte und meine kleine Outdoor Hexe. Es war eine herrliche Zeit mit dir unter einem Dach zu leben und ich danke dir für deine unglaublich kreativen Aufmerksamkeiten, die auch in den schwierigsten Situationen einen Lächeln aus mir herausgelockt haben. Ich danke dir für deine verrückten Ideen und dein großes Verständnis, wenn ich mal wieder an meiner Grenzen gekommen bin. Außerdem danke ich allen tollen Menschen mit denen ich eine wunderschöne und unvergessliche Zeit in der Villa Kunterbunt teilen durfte: Anna, Meike, Sandro, Simi, Seri, Robin, Cyril, Claudia, Adrian, Pano, Fabian, Judith, Mischi, Mirza und noch viele mehr. Ihr alle seid ein ganz besondere Inspirationsquelle gewesen und es war wirklich eine unglaublich besondere Zeit mit euch unter einem Dach zu leben.

Und nun zu meinen lieben Herdermern: Wie unglaublich viel Zeit uns inzwischen verbindet und wie stark wir doch immer noch miteinander verbunden sind. Ich 
danke euch dafür, dass ihr mir immer ein Gefühl von Rückhalt und zuhause gebt und der 23. Dezember jedes Jahr wieder aufs neue der schönste Tag des Jahres ist. Liebe Tete, dir an dieser Stelle, einen besonderen Dank. In der finalen Phase dich in der Nähe zu haben, hat mir wirklich unglaublich geholfen.

Und dann natürlich einen Dank an meine wundervolle Maaslos-Crew: Isi, Stella, Mattheus, David, Leo, Mira, Bob, Caro und Marc. Ihr seid für mich ein wundervolle Quelle der Inspiration und ein Haufen Lieblings-Nerds. Mich begeistert unser bunter Mix an Charakteren und unsere gemeinsam Passion und Ehrgeiz die Grenzen der Wissenschaft noch ein Stück zu erweitern. Ausserdem Ich freue mich, dass so manch ein Heavenly Hazel und maaslose Mengen an biertjes uns zusammengeschweisst haben und freue mich auf noch viele Pfingsten und andere Lebensereignisse mit euch zu feiern.

Taschi, Fee und Julia, meine Berliner Mädels und wieder ganz neuer Wind in meinem Leben. Wir haben uns zusammen an eine kreative Blase gewagt und manchen Post-it zusammen beschrieben an der D.School. Aber viel wichtiger, wir haben schnell gemerkt, dass uns noch viel mehr verbindet und ich freue mich so inspirierende, spannende, feinfühlige aber auch getriebene Mädels kennengelernt zu haben. Ich freue mich auf all unsere zukünftigen „kulturellen“ Ausflüge.

Among many other D.School friends, I would also like express my special thanks Amir for his huge support in translating this dissertation from Denglisch to proper English.

Dann möchte ich meinen Beachvolleyball Freunden danken - insbesondere Britta, Vicky und Lee - mit euch macht so ein Zock einfach am meisten Spass und man vergisst auch wirklich alles drum herum. Beachvolleyball hat mich in meinem Leben stark geprägt mir geholfen durchzuhalten, das Ziel (den Sieg) nicht aus den Augen zu verlieren, aber vor allem hat es mir so viel über das Leben gelernt und mich mit so vielen tollen Leuten in Verbindung gebracht. Danke Mila Superstar, dass du mich mit meinen 8 Jahren inspiriert hast und meine Leidenschaft für Beachvolley geweckt hast. Und Danke an alle tollen Menschen, mit denen ich diesen Sport teilen darf und die ich durch diesen Sport kennengelernt habe.

Ook is het al een tijdje geleden denk ik toch nog heel vaak aan de geweldige tijd in de Nationale DenkTank 2014. Wat een ontzettend inspirerende periode van all ons levens - op menselijk en inhoudelijke wijze. Ik voel me nog steeds heel erg verbonden - ook zijn onze paden ondertussen al weer iets verder uit elkaar gegroeid. Bedankt voor alles wat ik met en van jullie heb mogen leren. Vooral dat je elke kleine overwinning moet vieren.

Abschliessend möchte ich natürlich bei euch bedanken liebe Mami, lieber Papi, liebe Fia und liebe Omi, sowie auch dem Rest meiner Familie. Angefangen damit, 
dass es mich überhaupt gibt bis hin zum heutigen Tag. Danke, dass ich immer immer für mich da seid. Danke, dass ihr mir ermöglicht habt, diesen und so viele andere Meilensteine zu erreichen und danke, dass ihr mich immer wieder motiviert meine verrücktesten Träumen nachzueifern. Wenn ich jetzt ins Detail gehen würde, könnte es passieren, dass ich nochmal 200 Seiten fülle, daher belasse ich es bei diesen Worten. Es ist wahrscheinlich Zeichen genug, dass ein paar Freuentränen über meine Wange kullern, wenn ich an euch denke. Danke. 



\section{About the author}

Laura Mählmann was born July $18^{\text {th }} 1988$ in Oldenburg, Germany and grew up in Freiburg i. Brsg. After she obtained her high school diploma in 2008, she enrolled at Long Island University, NY in 2008 and 2009 on an athletic and academic scholarship to study International Business. In 2012, she successfully completed her Bachelor of Health Science (Major Movement Science and Minor Health Promotion and Education) and in 2014 her Master of Health Science Research and Global Health at Maastricht University, Netherlands. In 2014, she was invited to participate in the Dutch National ThinkTank on Big Data and its consequences for the Dutch society.

In the context of her $\mathrm{PhD}$, she conducted projects in collaboration with Prof. Ernst Hafen (ETH Zürich) and Prof. Effy Vayena (ETH Zürich), PD Dr. Serge Brand (UPK Basel) and Prof. Markus Gerber (University of Basel). She is supervised by Prof. Angela Brand, Faculty of Health, Medicine and Life Sciences (FHML) and Professorial Fellow at UNU-MERIT at Maastricht University. In addition to her work as a PhD student she is appointed as assistant researcher of Prof. Undine Lang at the Psychiatric Hospital (University of Basel) in Basel and she manages two projects related to brain-gut and depression, which aim to investigate to influence of probiotic supplementation or fecal microbial transfer on depressive symptoms in patients with severe Major Depressive Disorder (MDD).

Furthermore, she interned at the Academic Hospital Gemelli, Rome, on Health Technology Assessment (2011) and at the European Alliance of Personalized Medicine (EAPM) event at the European Parliament in Brussels (2012) on facilitating political and scientific collaborations. Finally she is currently enrolled in the Design Thinking Advanced Program at the "Hasso-Plattner Institute" in Potsdam. 



\section{Publications}

MähImann L, Reumann M, Evangelatos N., Brand A. Big Data for Public Health Policy Making: Policy Empowerment, Public Health Genomics doi: 10.1159/000486587.*

MähImann L, Gerber M, Furlano RI, Legeret C, Kalak N, Holsboer-Trachsler E, Brand S. Psychological wellbeing and physical activity in children and adolescents with inflammatory bowel disease compared to healthy controls. BMC Gastroenterol. 2017 Dec 12;17(1):160. doi: 10.1186/s12876-017-0721-7. *

Schee gen. Halfmann S, MähImann L, Leyens L, Reumann M, Brand A. Personalized Medicine: What's in it for Rare Diseases? Adv Exp Med Biol. 2017;1031:387-404. doi: 10.1007/978-3-319-67144-4_22.*

MähImann L, Gerber M, Furlano RI, Legeret C, Kalak N, Holsboer-Trachsler E, Brand S. Impaired objective and subjective sleep in children and adolescents with inflammatory bowel disease compared to healthy controls. Sleep Med. 2017 Nov;39:25-31. doi: 10.1016/j.sleep.2017.08.015. Epub 2017 Sep 21.*

MähImann L, Gerber M, Furlano RI, Legeret C, Kalak N, Holsboer-Trachsler E, Brand $\mathrm{S}$. Aerobic exercise training in children and adolescents with inflammatory bowel disease: Influence on psychological functioning, sleep and physical activity - an exploratory trial. Mental Health and Physical Activity, 2017.*

Zanchi D, Depoorter A, Egloff L, Haller S, Mählmann L, Lang UE, Drewe J, Beglinger C, Schmidt A, Borgwardt S. The impact of gut hormones on the neural circuit of appetite and satiety: A systematic review. Neurosci Biobehav Rev. 2017 Sep;80:457-475. doi: 10.1016/j.neubiorev.2017.06.013. Epub 2017 Jun 29.

MähImann L, Röcke C, Brand A, Hafen E, Vayena E. Attitudes towards personal genomics among older Swiss adults: An exploratory study. Appl Transl Genom. 2016 Feb 1;8:9-15. doi: 10.1016/j.atg.2016.01.009. eCollection $2016{\text { Mar. }{ }^{*}}^{*}$

MähImann L, Gerber M, Legeret C, Köhler H, Kalak N, Holsboer-Trachsler E, Brand S, Furlano R: Favorable impact of chronic aerobic exercise training (AET) on disease symptoms in children and adolescents with Inflammatory Bowel Disease (IBD). Submitted 2018.

* This publication is included in the dissertation. 
